\title{
Review of the braconid parasitoid subfamily Doryctinae (Hymenoptera, Braconidae) from the United Arab Emirates and Yemen
}

\author{
Sergey A. BELOKOBYLSKIJ ${ }^{\circledR 1,3, *}$ \& Cornelis VAN ACHTERBERG ${ }^{\bullet} 2$ \\ ${ }^{1}$ Zoological Institute, Russian Academy of Sciences, St. Petersburg 199034, Russia. \\ ${ }^{2}$ Naturalis Biodiversity Center, Postbus 9517, 2300 RA Leiden, The Netherlands. \\ ${ }^{3}$ Museum and Institute of Zoology, Polish Academy of Sciences, \\ Wilcza 64, Warszawa 00-679, Poland. \\ *Corresponding author: doryctes@gmail.com \\ 22Email: kees@vanachterberg.org \\ ${ }^{1}$ urn:Isid:zoobank.org:author:13EDEDEF-68BA-430B-8FC3-0096874859AB \\ ${ }^{2}$ urn:lsid:zoobank.org:author:D6374CF4-8F07-4FA8-8C55-9335FD19CECD
}

\begin{abstract}
A review of the braconid parasitoid subfamily Doryctinae of United Arab Emirates and Yemen is provided for the first time. The following genera are recorded in the southern Arabian Peninsula for the first time: tribe Doryctini - Hemispathius Belokobylskij \& Quicke, 2000 and Doryctes Haliday, 1836; tribe Spathiini - Parana Nixon, 1941 and Spathius Nees, 1819; tribe Hecabolini - Hemidoryctes Belokobylskij, 1992 and Parallorhogas Marsh, 1993; tribe Heterospilini - Heterospilus Haliday 1936; tribe Rhaconotini - Platyspathius Viereck, 1911 and Rhaconotinus Hedqvist, 1965. Sixteen species and one subspecies are described as new for science: Dendrosotinus (Gildoria) maculipennis Belokobylskij sp. nov., D. (G.) subelongatus Belokobylskij sp. nov., Doryctes (Neodoryctes) arrujumi Belokobylskij sp. nov., Parana arabica Belokobylskij sp. nov., Spathius alkadanus Belokobylskij sp. nov., S. austroarabicus Belokobylskij sp. nov., S. lahji Belokobylskij sp. nov., S. subafricanus Belokobylskij sp. nov., Hecabalodes maculatus Belokobylskij sp. nov., Platyspathius (Platyspathius) longicaudis Belokobylskij sp. nov., P. (P.) brevis Belokobylskij sp. nov., Rhaconotinus albosetosus Belokobylskij sp. nov., Rhaconotus brevicellularis Belokobylskij sp. nov., Rh. magniareolus Belokobylskij sp. nov., Rh. microexcavatus Belokobylskij sp. nov., Rh. vanharteni Belokobylskij sp. nov. and Hemidoryctes carbonarius postfurcalis Belokobylskij subsp. nov. Two new generic combinations are proposed: Hemispathius pilosus (Granger, 1949) comb. nov. (transferred from Doryctes) and Parallorhogas testaceus (Szépligeti, 1914) comb. nov. (transferred from Opius). Rhaconotus decaryi Granger, 1949 is here synonymised with Rh. menippus Nixon, 1939 (syn. nov.). A lectotype for Doryctes pilosus Granger, 1949 is designated. The following species are recorded for the UAE and/or Yemen for the first time: Dendrosotinus ferrugineus (Marshall, 1888), Hemispathius pilosus (Granger, 1949), Mimodoryctes proprius Belokobylskij, 2001, M. arabicus Edmardash, Gadallah \& Soliman, 2020, Spathius nixoni Belokobylskij \& Maetô, 2009, Hecabalodes anthaxiae Wilkinson, 1929, H. radialis Tobias, 1962, H. xylophagi Fischer, 1962, Parallorhogas testaceus (Szépligeti, 1914), Heterospilus (Eoheterospilus) rubrocinctus (Ashmead, 1905), Rhaconotinus menippus (Nixon, 1939), Rhaconotus arabicus
\end{abstract}


Belokobylskij, 2001, Rh. manolus Nixon, 1941, Rh. scirpophagae Wilkinson, 1927 and Rh. sudanensis Wilkinson, 1927.

Keywords. Hymenoptera, Braconidae, Oriental and Afrotropical regions, diversity, new species.

Belokobylskij S.A. \& van Achterberg C. 2021. Review of the braconid parasitoid subfamily Doryctinae (Hymenoptera, Braconidae) from the United Arab Emirates and Yemen. European Journal of Taxonomy 765: 1-143. https://doi.org/10.5852/ejt.2021.765.1479

\section{Introduction}

The studies focusing on the braconid parasitoid fauna of the dry territories of the southern Arabian Peninsula have been very fragmentary. Only recently, a few publications reviewing the subfamilies Agathidinae Haliday, 1833, Homolobinae van Achterberg, 1979, Microgastrinae Foerster, 1862 and Microtypinae Szépligeti, 1908 were published, mainly on the basis of large collection by Dr Antonius van Harten from the United Arab Emirates and Yemen (van Achterberg 2010, 2011, 2014; FernándezTriana \& van Achterberg 2017).

The origin of the Doryctinae Foerster, 1863 fauna from the Arabian Peninsula is mostly Afrotropical (especially Yemen), complimented with South Palaearctic and Oriental elements (especially the UAE). Until recently, few doryctine taxa were known from the Arabian Peninsula: Rhaconotus arabicus Belokobylskij, 2001 (Belokobylskij 2001a), Doryctophasmus ferrugineus (Granger, 1949) (Belokobylskij 2015) and Zombrus anisopus Marshall, 1897 (Marshall 1900; Fischer 1980). Recently, in the first contribution to the study of the Doryctinae fauna of the Farasan Archipelago (Red Sea, Saudi Arabia), the following genera were recorded for the first time (Edmardash et al. 2020): Dendrosotinus Telenga, 1941 (with D. ferrugineus (Marshall, 1888)), Hecabalodes Wilkinson, 1929 (with H. anthaxiae Wilkinson, 1929), Mimodoryctes Belokobylskij, 2001 (with M. proprius Belokobylskij, 2001 and M. arabicus Edmardash, Gadallah \& Soliman, 2020) and Neoheterospilus Belokobylskij, 2006 (with an undetermined male). A new species of Aivalykus Nixon, 1938 (tribe Ecphylini), A. microaciculatus Ranjith \& Belokobylskij, 2020, was described from United Arab Emirates and India (Ranjith et al. 2020). Finally, four new species belonging to Neoheterospilus (N. alkowdi Belokobylskij, 2020 and N. yemenus Belokobylskij, 2020) and Leluthia Cameron, 1887 (L. abnormis Belokobylskij, 2020 and L. brevitergum Belokobylskij, 2020), were also described from Yemen (Belokobylskij 2020a, 2020b).

The present paper records for the first time a significant number of Doryctinae genera and species for the Arabian Peninsula, mainly from Yemen and the United Arab Emirates (UAE). The keys to 17 genera recorded from this peninsula and to species of some genera (Spathius Nees, 1819, Platyspathius Viereck, 1911, Dendrosotinus, Rhaconotus Ruthe, 1854, Rhaconotinus Hedqvist, 1965, Neoheterospilus, Leluthia and Hecabalodes) are provided. We also describe 16 new species and one new subspecies and 15 previously recognized species are recorded from the UAE and/or Yemen for the first time.

\section{Material and methods}

This paper is mainly based on material collected in Yemen and the United Arab Emirates by Dr Antonius van Harten, occasionally with other collectors, during a period of about 20 years (1992-2011). The specimens were collected mostly by light and using Malaise traps throughout 23 localities in the United Arab Emirates (Al-Ajban, Bithnah, Fujairah, Hatta, NARC (= National Avian Research Center) near Sweihan, Sardjah, Sharhah x Khor Kalba, Sharjah, Sharjah Desert Park, SSW of ad-Dhaid, Wadi Maidaq, Wadi Safad, Wadi Shawkah, Wadi Wurayah) and Yemen (Al Kadan, Al Kowd, Al Lahima, Ar Rujum, Hammam 'Ali, Lahj, 12 km NW Manakhah, Sana'a, Ta'izz). This material comprises more than 1200 doryctine specimens. Information about localities and collection methods in the UAE can be found 
in van Harten (2009), those in Yemen are discussed by Lelej \& van Harten (2006). New distribution records presented in this paper are marked with an asterisk $\left(^{*}\right)$.

The terminology employed for morphological features and measurements follows Belokobylskij \& Maetô (2009). The wing venation nomenclature follows Belokobylskij \& Maetô (2009), with van Achterberg's (1993) terminology shown in parentheses. The suture between the second and third metasomal tergites is named here as the 'second suture'; praescutellar depression is the sulcus between mesoscutum and scutellum; acrosternite is the coarsely sclerotised basal part of sternite of the first metasomal segment.

Specimens were examined using an Olympus SZX12 stereo microscope. Photographs were taken with a Leica IC 3D digital camera mounted on a Leica MZ16 microscope and using the Leica Application Suite imaging system (Museum and Institute of Zoology, Warsaw, Poland), and with a Canon EOS 70D digital camera mounted on an Olympus SZX10 microscope (Zoological Institute RAS, St Petersburg, Russia). Digital pictures were enhanced and plates were edited using Adobe Photoshop.

The specimens examined for this study, including type material, are deposited in the following collections:

$\mathrm{BMNH}=$ The Natural History Museum, London, UK

MNBG = Museum für Naturkunde, Leibniz-Institut für Evolutions- und Biodiversitätsforschung, Berlin, Germany

MNHN $=$ Muséum national d'histoire naturelle, Paris, France

RMNH $=$ Naturalis Biodiversity Center, Leiden, The Netherlands

ZISP $=$ Zoological Institute of the Russian Academy of Sciences, St Petersburg, Russia

\section{Abbreviations}

$\mathrm{Od}=$ maximum diameter of the posterior ocellus

$\mathrm{OOL}=$ ocular-ocellar line, shortest distance between posterior ocellus and eye

$\mathrm{POL}=$ postocellar line, shortest distance between posterior ocelli

Most of the Arabian specimens studied are deposited in RMNH, with some duplicates in ZISP.

\section{Results}

Class Insecta Linnaeus, 1758

Order Hymenoptera Linnaeus, 1758

Family Braconidae Nees, 1811

Subfamily Doryctinae Foerster, 1863

\section{Key to genera of Doryctinae in the Arabian Peninsula}

1. First metasomal tergite distinctly petiolate; acrosternite of first segment long, $0.5-0.9$ times as long as tergite (Figs 14I, 15C-D, 19B-C, 23B, D)

- First metasomal tergite not petiolate or subpetiolate; acrosternite of first segment short or slightly elongate, $0.1-0.4$ times as long as tergite (Figs 2B-C, 8B-C, 10B, D, 13C-D, 27B-C, 48C-D) ….. 4

2. Laterotergites (epipleura) of second and third tergites fused (Figs 17D, 19C, 21D, 23D). First metasomal tergite (petiole) usually more or less abruptly dilated at apex (Figs 16K, 18H, 23B). Fore and middle femora without blister-like swelling Spathius Nees, 1819

- Laterotergites (epipleura) of second and third tergites separated (Figs 15D, 40C, 42C). First metasomal tergite (petiole) gradually widened from spiracles to apex, never abruptly dilated at apex (Figs 14J, 39J, 42B). Fore and middle femora with a blister-like swelling near base above. Almost always dark 
brown specimens (Figs 14A, 39A, 41A). Hind tibiae never pale basally (Figs 14I, 39G, 41J). Second to fourth tergites always with distinctly separated laterotergites (Figs 15D, 40C, 42C). Fore wing strongly dappled with black and white. Medial vein (M+CU1) very strongly curved (Figs 15A, 40A, 42A) ... 3

3. Nervulus (m-cu) of fore wing widely antefurcal (Fig. 15A). Infuscate part of fore wing proximal to basal hyaline fascia completely without pubescence (Fig. 15A). Basal vein (1r-m) of hind wing absent, costal vein (1-SC+R) fused just with mediocubital vein (1-M) (Fig. 15A). Precoxal sulcus almost absent (Fig. 14H). Acrosternite of petiole considerably longer than half of first tergite (Fig. 15D)

Parana Nixon, 1943

- Nervulus (m-cu) of fore wing usually postfurcal or rarely interstitial (Figs 40A, 42A). Infuscate part of fore wing proximal to basal hyaline fascia always pubescent (Figs 40A, 42A). Basal vein (1r-m) of hind wing present, costal vein (1-SC+R) not fused just with mediocubital vein (1-M) (Figs 40A, 42A). Precoxal sulcus present, but often almost indistinct (Figs 39I, 41H). Acrosternite of petiole rather short, usually shorter than half of petiole tergite (Figs 39K, 42C) (see also couplet 7)

Platyspathius Viereck, 1911

4. Hind wing without nervellus (cu-a); submedial (subbasal) cell of hind wing absent (Fig. 64A). Labial palpus 3-segmented. First flagellar segment distinctly shorter than second segment (Fig. 63C). Second radiomedial vein (r-m) of fore wing absent (Fig. 64A). Brachial (subdiscal) cell closed distally and basally; nervulus (cu-a) present (Fig. 64A) Aivalykus Nixon, 1938

- Hind wing with nervellus (cu-a); submedial (subbasal) cell of hind wing present and closed distally (Figs 2A, 8A, 10A, 27A, 44A). Labial palpus 4-segmented

5. Brachial (subdiscal) cell of fore wing closed apico-posteriorly; brachial vein (CU1b) present (Figs 2A, 8A, 10A, 11F, 40A, 44A, 50A). Medial (basal) cell of hind wing usually wide, more or less distinctly widened toward apex (Figs 2A, 8A, 10A, 11F, 40A, 44A, 50A). Usually second ( $\mathrm{r}-$ m) (except for Mimodoryctes: Fig. 13A-B) and always first radiomedial vein (2-SR) of fore wing present (Figs 2A, 8A, 10A, 11F, 40A, 44A, 50A). Hind wing of males without pterostigma-like sclerotisation

- Brachial (subdiscal) cell of fore wing open apico-posteriorly; brachial vein (CU1b) absent (Figs 27A; 29A; 35A, 37A; B; 38F). Medial (basal) cell of hind wing narrow, usually not or weakly widened toward apex (Figs 27A; 29A; 35A, 37A; B; 38F). Often second (r-m) or first (2-SR) radiomedial vein of fore wing absent (Figs 27A; 29A; 35A, 37A; B; 38F). Hind wing of males often with pterostigma-like sclerotisation

6. Parallel vein (CU1a) interstitial to cubital vein (2-CU1) or rarely arising distinctly behind middle of vein (3-CU1), closed brachial (subdiscal) cell distally (Figs 2A, 4A, 6A, 40A, 42A, 44A, 50A). Recurrent vein (m-cu) of fore wing postfurcal (Figs 2A, 4A, 6A, 40A, 42A, 44A, 50A) .............. 7

- Parallel vein (CU1a) arising distinctly before middle of vein (3-CU1), closed brachial (subdiscal) cell distally (closely to posterior margin of wing) (Figs 8A, 10A, 11F, 13A). Recurrent vein (m-cu) of fore wing antefurcal (Figs 8A, 10A, 11F, 13A) .

7. Hind tibia with row of sparse thick spines on dorsal surface. First flagellar segment smooth and concave on one side, convex and rugulose at least on basal half of opposite side. Acrosternite of first segment elongated, $0.35-0.40$ times as long as tergite. Hind wing of male with pterostigma-like sclerotisation at least on part of costal vein Doryctophasmus Enderlein, 1912

- Hind tibia without row of spines on dorsal surface (Figs 7L, 39G, 41J, 43J, 49G). First flagellar segment smooth and more or less convex on both sides (Figs 7F, 12G, 39F, 41F, 43E, 47E). Acrosternite of first segment not elongate (except Platyspathius: Figs 39K, 42C), 0.25-0.30 times 
as long as tergite (Figs $8 \mathrm{C}, 44 \mathrm{C}, 50 \mathrm{C}$ ). Hind wing of male without pterostigma-like sclerotisation .

8. First metasomal tergite narrow and elongate, subpetiolate, 1.6-1.8 times longer than its posterior width; its acrosternite elongated, usually 0.3-0.4 times as long as tergite (Figs 39K, 40C, 42C) (see also couplet 3)

Platyspathius Viereck, 1911

- First metasomal tergite wide and short or relatively short, sessile, 1.0-1.3 times longer than its posterior width; its acrosternite not elongated, usually $0.1-0.2$ times as long as tergite (Figs $2 \mathrm{~B}-\mathrm{C}$, 4B-C, 6B-C, 44B-C, 48C-D)

9. At most second metasomal tergite and sometimes third one with separated laterotergites (Figs $2 \mathrm{C}$, $4 \mathrm{C}, 6 \mathrm{C}$ ). Base of fourth to sixth tergites without transverse furrows (Figs 2B, 4B, 6B). Metasomal tergites behind third one always smooth (Figs 2B, 4B, 6B). Fifth and sixth metasomal tergites not enlarged, not longer than proceeding tergite and not concealing succeeding tergites (Figs 2B, 4B, $6 \mathrm{~B})$. Femora of all legs without dorsal tubercles (Figs 1H, 3H, 5G)

Dendrosotinus Telenga, 1941

- Second to fifth or sixth metasomal tergites with separated laterotergites (Figs 44C, 46C, 48C, $50 \mathrm{C}, 52 \mathrm{C}, 54 \mathrm{C}, 56 \mathrm{C})$. Base of fourth to sixth tergites with deep and crenulate transverse furrows (Figs 44B, 46B, 48B, 50B, 52B, 54B, 56B). Metasomal tergites behind third one sculptured at least anteriorly (Figs 44B, 46B, 48B, 50B, 52B, 54B, 56B). Fifth or sixth metasomal tergites enlarged, usually longer than proceeding tergite and concealing succeeding tergites (Figs 44B, 46B, 48B, 50B, 52B, 54B, 56B). Femora of fore, middle and sometimes hind legs with dorsal protuberances (Figs 43G, 45F, 49G, 51I, 53H, 55I, 61F)

10. Metasoma with only five tergites visible dorsally (following tergites hidden under fifth one); fifth tergite usually distinctly longer than previous (fourth) tergite (Figs 48C, 50B, 52B, 54D, 56B, 58C, $60 \mathrm{~B}, 62 \mathrm{C})$

Rhaconotus Ruthe, 1854

- Metasoma with six or seven tergites visible dorsally; sixth tergite longer or not longer than previous (fifth) tergite (Figs 44B, 46B) Rhaconotinus Hedqvist, 1965

11. Acrosternite of first segment elongated, 0.4-0.6 times as long as first tergite (Figs 10C- D). Hind coxa without or with very small (almost invisible) basoventral tubercle (Fig. 9F). Second and third metasomal tergites with two lateral subparallel furrows (Figs 9I, 10B). Mesoscutum granulate or granulate-coriaceous (Fig. 9G). Propodeum without delineated areas (Fig. 9G)

Hemispathius Belokobylskij \& Quicke, 2000

- Acrosternite of first segment short, not elongated, 0.25-0.30 times as long as first tergite (Figs 8C, 13D). Hind coxa with distinct basoventral tubercle (Figs 7K, 12G). Second and third metasomal tergites usually without lateral furrows (Figs $8 \mathrm{~B}, 11 \mathrm{G}, 13 \mathrm{C}$ )

12. Parallel vein (CU1a) of fore wing arising distinctly before middle of distal margin of brachial (subdiscal) cell (Fig. 8A). Second radiomedial vein (r-m) of fore wing always present (Fig. 8A). Third segment of labial palpus long (Fig. 7D). Dorsal lobe of pronotum more or less distinctly convex (Fig. 7I). Posterior end of precoxal sulcus usually with rounded depression

Doryctes Haliday, 1836

- Parallel vein (CU1a) of fore wing arising from middle of distal margin of brachial (subdiscal) cell (Figs 11F, 13A-B). Second radiomedial vein ( $\mathrm{r}-\mathrm{m})$ of fore wing absent (Figs 13A-B) or present (Fig. 11F). Third segment of labial palpus distinctly shortened. Dorsal lobe of pronotum absent (Fig. 12J). Posterior end of precoxal sulcus without rounded depression (Fig. 12J) Mimodoryctes Belokobylskij, 2001 
13. First radiomedial vein (2-SR) of fore wing absent or largely unsclerotised (Fig. 38F). Second radiomedial vein $(\mathrm{r}-\mathrm{m})$ of fore wing always present (Fig. 38F)

- First radiomedial vein (2-SR) of fore wing present and mainly well sclerotised (Figs 27A, 29A, 31A, 33A, 35A-B; 37A). Second radiomedial vein ( $\mathrm{r}-\mathrm{m}$ ) of fore wing present (Figs 35A- B, 37A) or absent (Figs 27A, 29A, 31A, 33A)

14. Apex of female vipositor transformed, upcurved, constricted subposteriorly, then widened and usually strongly narrowed towards apex; apex of ovipositor sheath distinctly widened. Second metasomal tergite often with basal area more or less distinctly delineated by furrow

Neoheterospilus Belokobylskij, 2006

- Apex of of female ovipositor not transformed, not upcurved and rather evenly narrowed towards apex; apex of ovipositor sheath not or sometimes only weakly widened (Fig. 38A). Second metasomal tergite always without basal area delineated by furrow (Fig. 38H) ........ Heterospilus Haliday, 1836

15. Hind coxa without basoventral tooth (Fig. 36G). Fore wing distinctly maculate (Fig. 37A). Nervulus (cu-a) of fore wing strongly postfurcal (Fig. 37A). - Vertex and mesopleuron densely granulate (Fig. 36C, I). Hind wing of male with large simple pterostigma-like sclerotisation at costal vein ....

Hemidoryctes Belokobylskij, 1992

- Hind coxa with distinct basoventral tooth (Figs 27C, 32H, 34A). Fore wing hyaline or only sometimes partly faintly infuscate (Figs 27A, 29A, 31A, 33A, 35A-B). Nervulus (cu-a) of fore wing more or less weakly postfurcal (Figs 27A, 29A, 31A, 33A, 35A-B)

16. Vertex usually smooth, rarely with fine aciculation, always without granulation (Fig. 34C, H). Mesonotum without granulation, mainly smooth; mesopleuron smooth in lower half (Fig. 34I, J). Second radiomedial vein $(\mathrm{r}-\mathrm{m})$ of fore wing always present (Fig. 35A-B). Recurrent vein (m-cu) of hind wing usually weakly curved towards apex of wing (Fig. 35B); if sometimes almost straight, then perpendicular to mediocubital vein (1-M). Propodeum with large areola delineated by distinct carinae (Fig. 34I). Hind wing of male without pterostigma-like sclerotisation

Parallorhogas Marsh, 1993

- Vertex granulate and usually with additional undulate transverse striae or reticulation (Figs 26B, 28D, 30C, 32B). Mesonotum granulate and often with additional rugosity; mesopleuron granulate in lower half (Figs 26E-F, 28G-H, 30D-E, 32F-G). Second radiomedial vein ( $\mathrm{r}-\mathrm{m})$ of fore wing often absent (Figs 27A, 29A, 31A, 33A), but sometimes present. Recurrent vein (m-cu) of hind wing always curved towards base of wing (Figs 27A, 29A, 31B, 33A). Propodeum basically without areola delineated by carinae (Figs 26E, 28G, 30E, 32G). Hind wing of male with pterostigma-like sclerotisation 17

17. Radial (marginal) cell or fore wing not or only weakly shortened; metacarpus (1-R1) usually not or only slightly shorter than pterostigma Leluthia Cameron, 1887

- Radial (marginal) cell or fore wing distinctly shortened; metacarpus (1-R1) distinctly shorter than pterostigma (Figs 27A, 29A, 31A, 33A) Hecabalodes Wilkinson, 1929

Tribe Doryctini Foerster, 1863

Genus Dendrosotinus Telenga, 1941

\section{Type species}

Dendrosoter ferrugineus Marshall, 1888, by original designation.

\section{Remarks}

This genus consists of two subgenera, Dendrosotinus s. str. and Gildoria Hedqvist, 1974. The subgenus Gildoria is here recorded from the Arabian Peninsula for the first time. 


\section{Distribution}

Afrotropical, Oceanic, Oriental and Palaearctic regions.

\section{Key to species of the genus Dendrosotinus from Arabia Peninsula}

1. Third antennal segment (especially of female) weakly widened, more or less depressed and anteriorly sculptured (Fig. 1C, E). Parallel vein (m-cu) of fore wing subinterstial; brachial (subdiscal) cell moderately wide (Fig. 2A). (Subgenus Dendrosotinus s. str.)

D. (D.) ferrugineus (Marshall, 1888)

- Third antennal segment slender, cylindrical and anteriorly smooth (Figs 3C, 5B). Parallel vein (m$\mathrm{cu}$ ) of fore wing distinctly postfurcal; brachial (subdiscal) cell narrow (Figs 4A, 6A-B), (Subgenus Gildoria Hedqvist, 1974)

2. Transverse diameter of eye (dorsal view) 1.9-2.3 times longer than temple (Fig. 3E). Malar space 0.4 times maximum diameter of eye (Fig. 3B). Length of first tergite 1.1-1.2 times its maximum posterior width (Figs 3I, 4B). Second tergite without sublateral depression (Figs 3I, 4B). Ovipositor sheath 0.5-0.6 times as long as metasoma, 0.4-0.5 times as long as fore wing (Fig. 3A). Body mainly light reddish brown or reddish brown, darkened dorsally (Fig. 3A)

D. (G.) maculipennis Belokobylskij sp. nov.

- Transverse diameter of eye (dorsal view) 1.2-1.4 times longer than temple (Fig. 5C). Malar space $0.55-0.60$ times maximum diameter of eye (Fig. 5B). Length of first tergite $1.3-1.4$ times its maximum posterior width (Figs 5F, 6B). Second tergite with very shallow, subparallel, sublateral and almost straight narrow depression in anterior half (Fig. 6B). Ovipositor sheath 0.9-1.0 times as long as metasoma, $0.70-0.75$ times as long as fore wing (Fig. 5A). Body mainly brownish yellow to yellow partly (Fig. 5A)

D. (G.) subelongatus Belokobylskij sp. nov.

Dendrosotinus (Dendrosotinus) ferrugineus (Marshall, 1888)

Figs $1-2$

Dendrosotinus ferrugineus Marshall, 1890: 247.

Dendrosotinus ferrugineus - Belokobylskij 1983: 1845. — Belokobylskij \& Tobias 1986: 39. — Yu et al. 2016. — Edmardash et al. 2020: 45.

\section{Material examined}

UNITED ARAB EMIRATES • 2 우; "United Arab Emirates, Sharjah Desert Park (1081), light tr.,

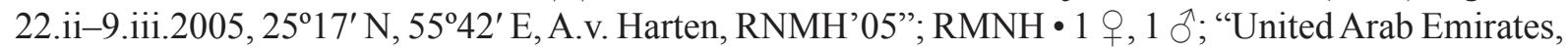

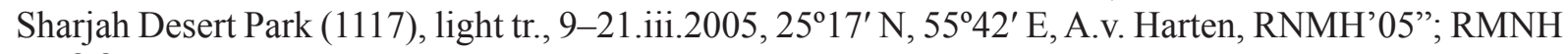

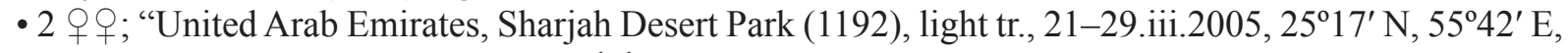
A.v. Harten, RNMH'05"; RMNH • 3 đ̋

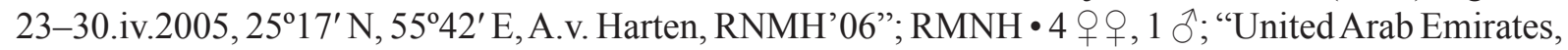

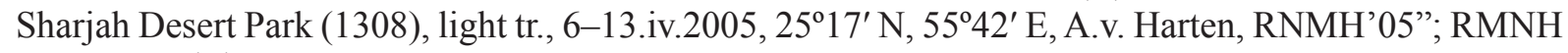

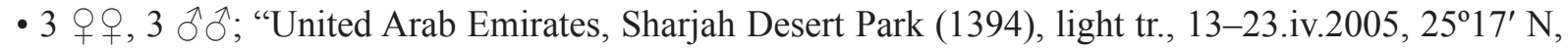

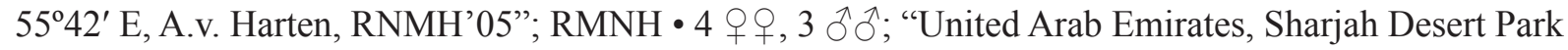

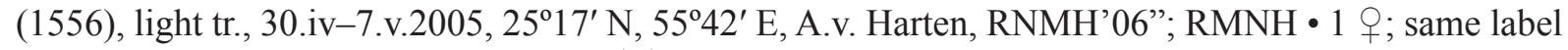

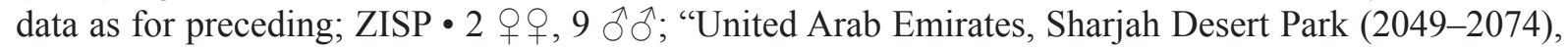

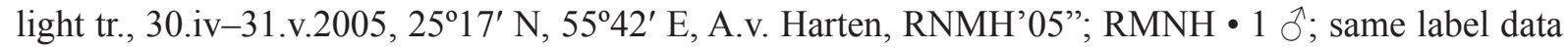
as for preceding; ZISP • 7 우, $22 \overbrace{}^{\Uparrow} \delta^{\lambda}$; "United Arab Emirates, Sharjah Desert park (2517), light trap,

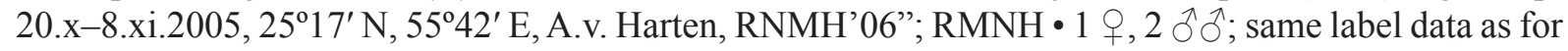
preceding; ZISP • 2 우; “United Arab Emirates, Sharjah Desert Park (3692-3697), light tr., 11.xii.2005-

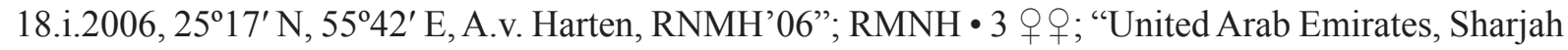




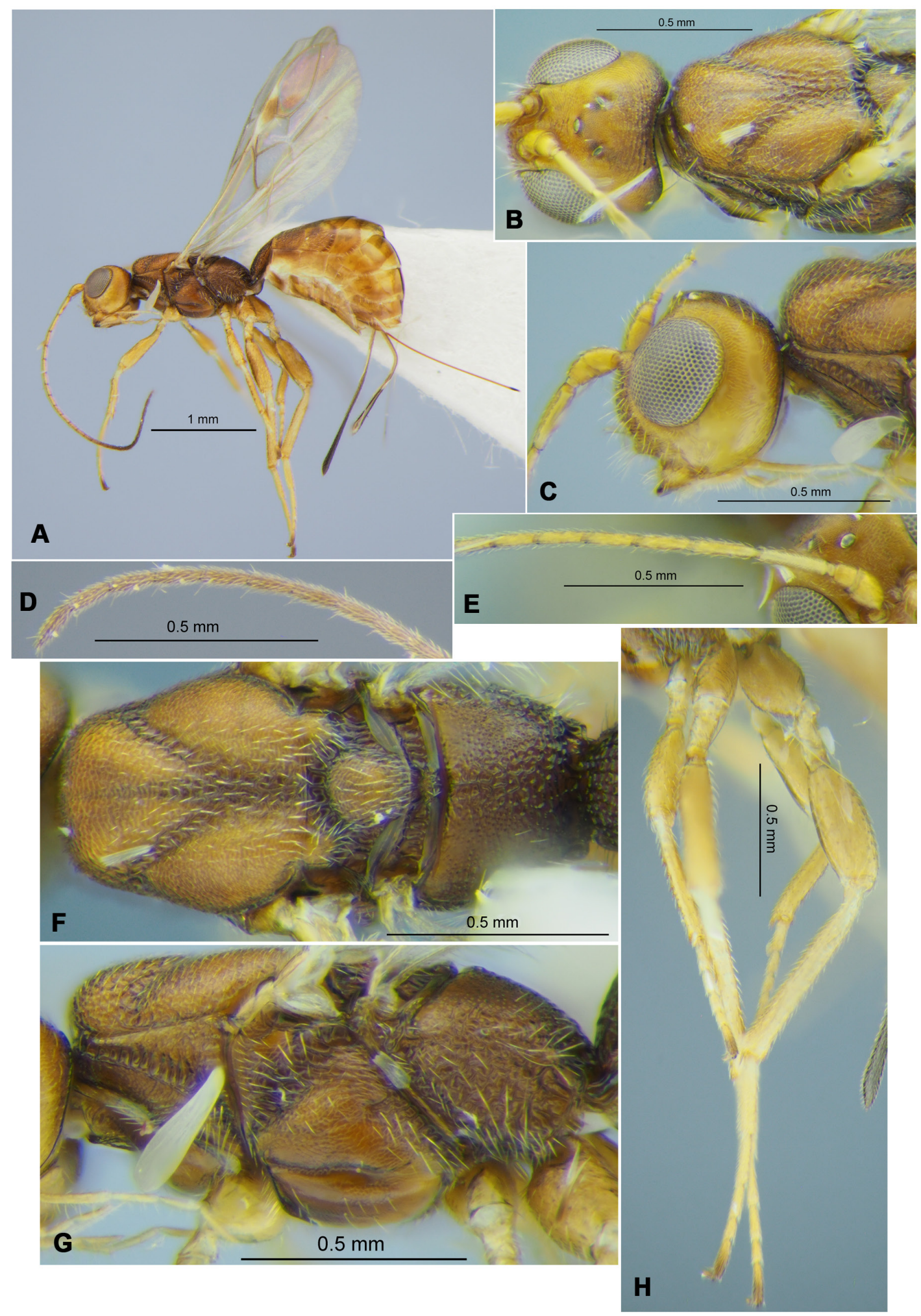

Fig. 1. Dendrosotinus (Dendrosotinus) ferrugineus (Marshall, 1888), $q$ (RMNH). A. Habitus, lateral view. B. Head and mesoscutum, dorsal view. C. Head and mesoscutum, lateral view. D. Apical segments of antenna. E. Basal segments of antenna. F. Mesosoma, dorsal view. G. Mesosoma, lateral view. H. Hind leg. 

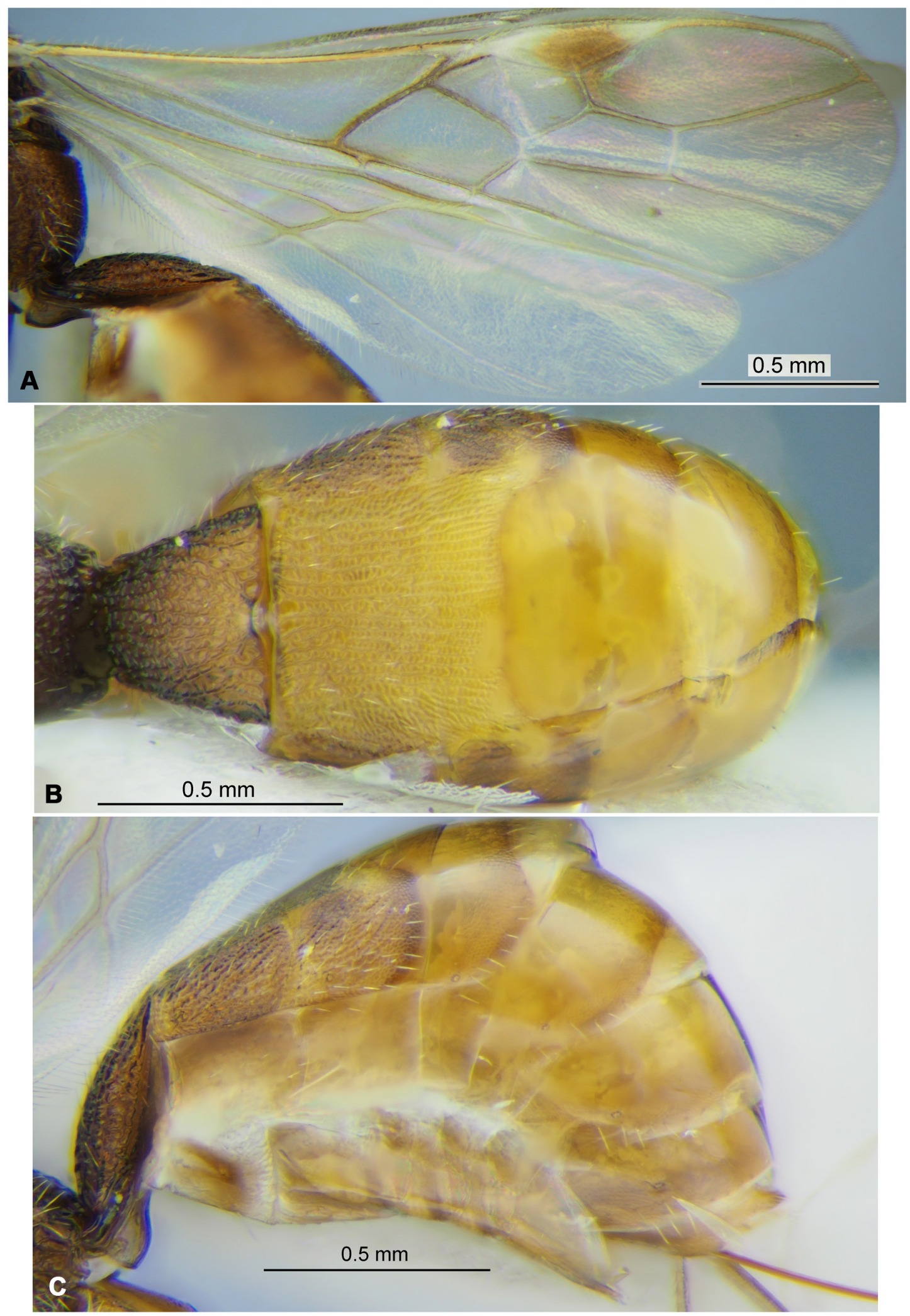

Fig. 2. Dendrosotinus (Dendrosotinus) ferrugineus (Marshall, 1888), $q$ (RMNH). A. Wings. B. Metasoma, dorsal view. C. Metasoma, lateral view. 


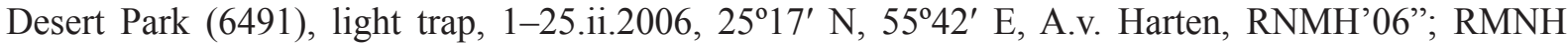
- 3 우우, 1 đ; "United Arab Emirates, Sharjah Desert Park (5704-5740), light tr., 25.ii-25.iii.2006, $25^{\circ} 17^{\prime} \mathrm{N}, 55^{\circ} 42^{\prime}$ E, A.v. Harten, RNMH'06”; RMNH • 1 \% ; same label data as for preceding; ZISP - 1 ㅇ; “United Arab Emirates, Sharjah Desert Park (3908-3949), light tr., 20-21.iv.2006, 25¹7' N, $55^{\circ} 42^{\prime}$ E, M. Fibiger, RNMH'06”; RMNH • 1 +; "United Arab Emirates, Al-Ajban (4317), Malaise tr.,

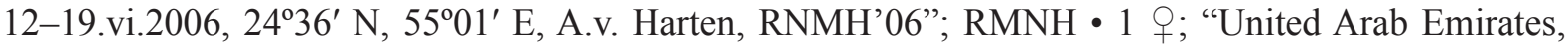

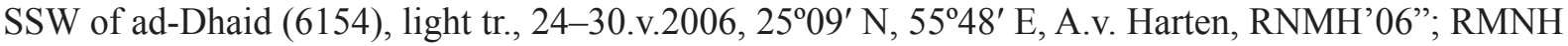
- 1 O; " 'United Arab Emirates, Wadi Safad (5867-5902), at light, 31.i-21.ii.2006, 2513' N, 56¹9' E, A.v. Harten, RNMH'06"; RMNH • 1 क; "United Arab Emirates, NARC near Sweihan (1193), at light,

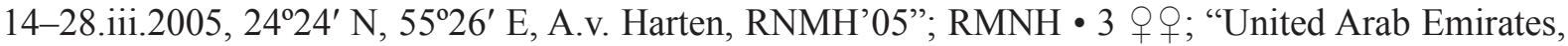

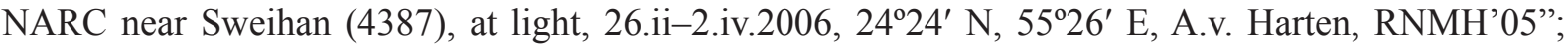
RMNH • 1 Oे; "United Arab Emirates, Sharhah x Khor Kalba (6319-6320), light trap, 16-31.i.2006, A.v. Harten, RNMH'06"; RMNH.

\section{Distribution}

Armenia, Azerbaijan, former Yugoslavia, France, Greece, Israel, Italy, Russia (North Caucasus), Saudi Arabia, Spain, Turkey, *UAE.

Dendrosotinus (Gildoria) maculipennis Belokobylskij sp. nov. urn:lsid:zoobank.org:act:FE8312CB-C5B4-41B3-955F-6276D64631A6

Figs 3-4

\section{Comparative diagnosis}

This new species is similar to D. (G.) grandis (Fahringer, 1930) known only from Spain, but differs from it by the mediocubital vein (M+CU1) of fore wing distinctly curved in apical half (nearly straight in $D$. grandis), vertex densely irregularly transversely striate with dense and numerous rugosity (mainly granulate in D. grandis), radial vein (r) arising behind middle of pterostigma (from middle in D. grandis), brachial (subdiscal) cell closed distally distinctly before recurrent $(\mathrm{m}-\mathrm{cu})$ vein (weakly before vein in $D$. grandis), second radiomedial (submarginal) cell not narrowed distally (distinctly narrowed in D. grandis) and hind basitarsus short and thick (long and slender in D. grandis).

\section{Etymology}

Name derived from a combination of 'maculis' (Latin for 'spotted') and 'pennis' (Latin for 'plumage') because of the spotted colouration of the fore wing.

\section{Material examined}

\section{Holotype}

YEMEN • +; "Yemen, Al Kowd, ii.2001, light tr., no 5484, A.v. Harten \& S. Al Haruri. RMNH'01"; RMNH.

\section{Paratypes}

YEMEN • 3 우; “Yemen, Al Kowd, ii.2000, light tr., no 4431, A.v. Harten \& S. Al Haruri. RMNH'01"; RMNH • 1 क; same label data as for preceding; ZISP • 1 q; "Yemen (no 2989), Ta'izz, light trap, 1-3. iv.1998, A.v. Harten \& Ahmad Ahwad, RMNH'98”; RMNH • 1 q; "Yemen, Ta'izz, x.1999, light tr., no 4249 A.v. Harten \& A. Awad, RMNH’01”; RMNH.

\section{Description}

Female

Measurements. Body length 2.2-2.8 mm; fore wing length $1.8-1.9 \mathrm{~mm}$. 
HeAD. Head width (dorsal view) 1.5-1.6 times its median length, 1.2 times width of mesoscutum. Head behind eyes (dorsal view) rather distinctly convex-roundly narrowed. Transverse diameter of eye (dorsal view) 1.9-2.3 times longer than temple. Ocelli small, arranged in triangle with base 1.3-1.4 times its sides. POL 1.7-2.0 times Od, 0.7-0.8 times OOL. Eye glabrous, its maximum diameter 1.2 times minimum diameter. Malar space 0.4 times maximum diameter of eye, 0.9-1.0 times basal width of mandible. Face convex, its width 0.9-1.0 times maximum diameter of eye, 1.3-1.4 times height of face and clypeus combined. Clypeal suture almost complete, distinct laterally and fine to very fine dorsally. Hypoclypeal depression medium-sized and rounded, its width 0.7 times shortest distance from edge to eye, $0.30-0.35$ times width of face. Occipital carina dorsally complete and not broken toward ocellar

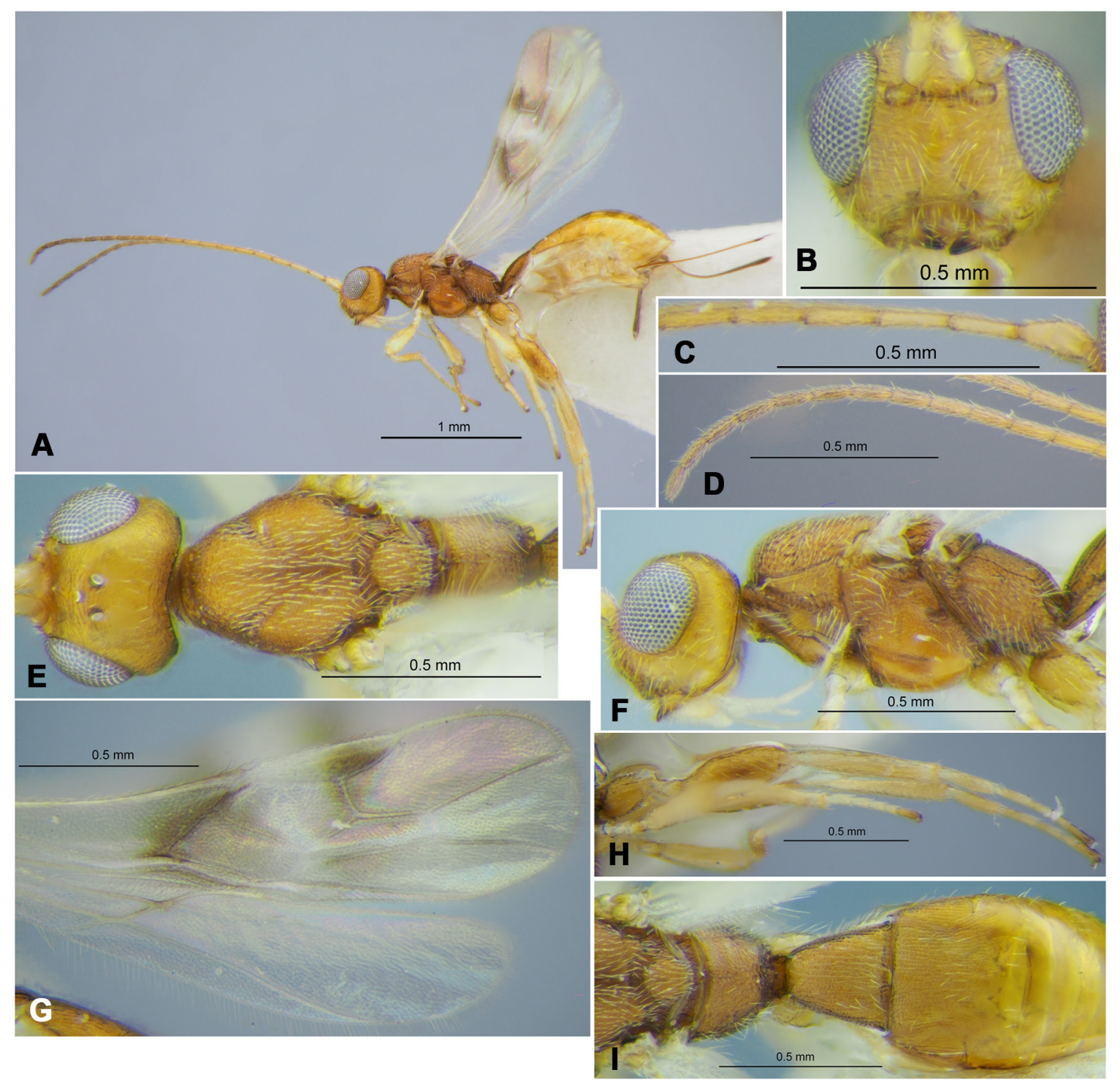

Fig. 3. Dendrosotinus (Gildoria) maculipennis Belokobylskij sp. nov., holotype, $q$ (RMNH). A. Habitus, lateral view. B. Head, front view. C. Basal segments of antenna. D. Apical segments of antenna. E. Head and mesosoma, dorsal view. F. Head and mesosoma, lateral view. G. Wings, distal part. H. Hind leg. I. Propodeum and basal part of metasoma. 
triangle, not reaching below hypostomal carina and obliterated a short distance before mandible base. Hypostomal flange distinct.

AnTENNA. Antenna rather slender, filiform, 21-22-segmented, 0.9-1.0 times as long as body. Scape 1.31.5 times longer than maximum width. First flagellar segment 4.2-4.5 times longer than apical width, about as long as second segment. Penultimate segment 3.0-3.2 times longer than width, 0.6-0.9 times as long as first segment, $0.9-1.0$ times as long as apical segment; the latter weakly acuminate apically and without spine.

Mesosoma. Mesosoma not depressed, its maximum length 1.7-1.8 times its maximum height. Pronotal carina distinct, situated submedially. Mesoscutum (lateral view) high, subvertical roundly elevated above pronotum, its median lobe (dorsal view) protruding forwards, convex anteriorly and without anterolateral corners, with complete, fine, rather deep posteriorly and very shallow anteriorly longitudinal median furrow; mesoscutum in dorsal view 1.0-1.1 times as long as wide. Notauli almost complete, deep in anterior half and shallow in posterior half, crenulate. Prescutellar depression rather deep, same length medially and laterally, evenly curved, with $6-8$ carinae, almost smooth between carinae, $0.25-0.30$ times as long as scutellum. Scutellum convex, with distinct lateral carinae, 1.1-1.2 times longer than maximum anterior width. Subalar depression rather wide, shallow, rugulose-reticulate. Precoxal sulcus rather deep and wide, densely reticulate, running along anterior half of lower part of mesopleuron. Metanotum with low, wide and distally acuminate medio-dorsal tubercle (lateral view). Metapleural flange (lobe) wide, short, subrounded distally. Propodeum weakly convex, with small lateral tubercles.

WINGS. Fore wing 3.5-3.6 times longer than wide. Pterostigma rather narrow, 4.0-4.5 times longer than its maximum width. Radial vein (r) arising behind middle of pterostigma, from basal $0.55-0.60$. Radial (marginal) cell not shortened, metacarpus (1-R1) 1.0-1.1 times as long as pterostigma. Second radial abscissa (3-SR) 1.7-2.0 times longer than first abscissa (r) and forming with it a weakly obtuse angle, $0.50-0.55$ times as long as straight third abscissa (SR1), 1.1 times longer than straight first radiomedial vein (2-SR). Second radiomedial (submarginal) cell not narrowed distally, its length 3.2-3.4 times maximum width, 1.8-2.0 times length of narrow brachial (subdiscal) cell. Second abscissa of medial vein $(2-\mathrm{SR}+\mathrm{M})$ short, $0.35-0.40$ times as long as recurrent vein $(\mathrm{m}-\mathrm{cu})$. Third abscissa of medial vein (2-M) almost straight. Nervulus (cu-a) short, distinct, strongly postfurcal, distance between basal vein (1-M) and nervulus (cu-a) 1.5-1.8 times nervulus length. Mediocubital vein (M+CU1) rather evenly and distinctly curved to longitudinal anal vein (1-1A). Narrow brachial (subdiscal) cell closed apically distinctly before recurrent vein (m-cu), apically reclivous and straight. Parallel vein (CU1a) interstitial. Hind wing 4.8-5.3 times longer than its maximum width. First costal abscissa $(\mathrm{C}+\mathrm{SC}+\mathrm{R})$ 0.7-1.0 times as long as second abscissa (1-SC+R). First abscissa of mediocubital vein (M+CU) 0.6-0.7 times as long as second and third abscissae (1-M) combined. Recurrent vein (m-cu) rather long, unsclerotised and not pigmented, distinctly antefurcal, strongly oblique towards base of wing.

Legs. Hind coxa without basoventral tubercle, but with distinct convex basoventral corner, 1.4-1.6 times longer than maximum width. Hind femur elongate-oval, 2.5-2.9 times longer than wide. Hind tibia without spines on outer distal margin. Hind tarsus 1.0-1.1 times longer than hind tibia. Hind basitarsus thickened, $0.50-0.55$ times as long as remaining segments combined. Second segment of hind tarsus $0.60-0.65$ times as long as basitarsus, 1.2-1.4 times longer than narrow fifth segment (without pretarsus).

Metasoma. Metasoma 1.2-1.3 times longer than mesosoma and head combined. First metasomal tergite rather long and wide, with very weak spiracular protuberances in anterior third, distinctly and almost linearly widened from base to apex. Length of first tergite 1.1-1.2 times its maximum posterior width; posterior width 2.5-2.6 times its minimum anterior width. Median length of second tergite 0.6-0.7 

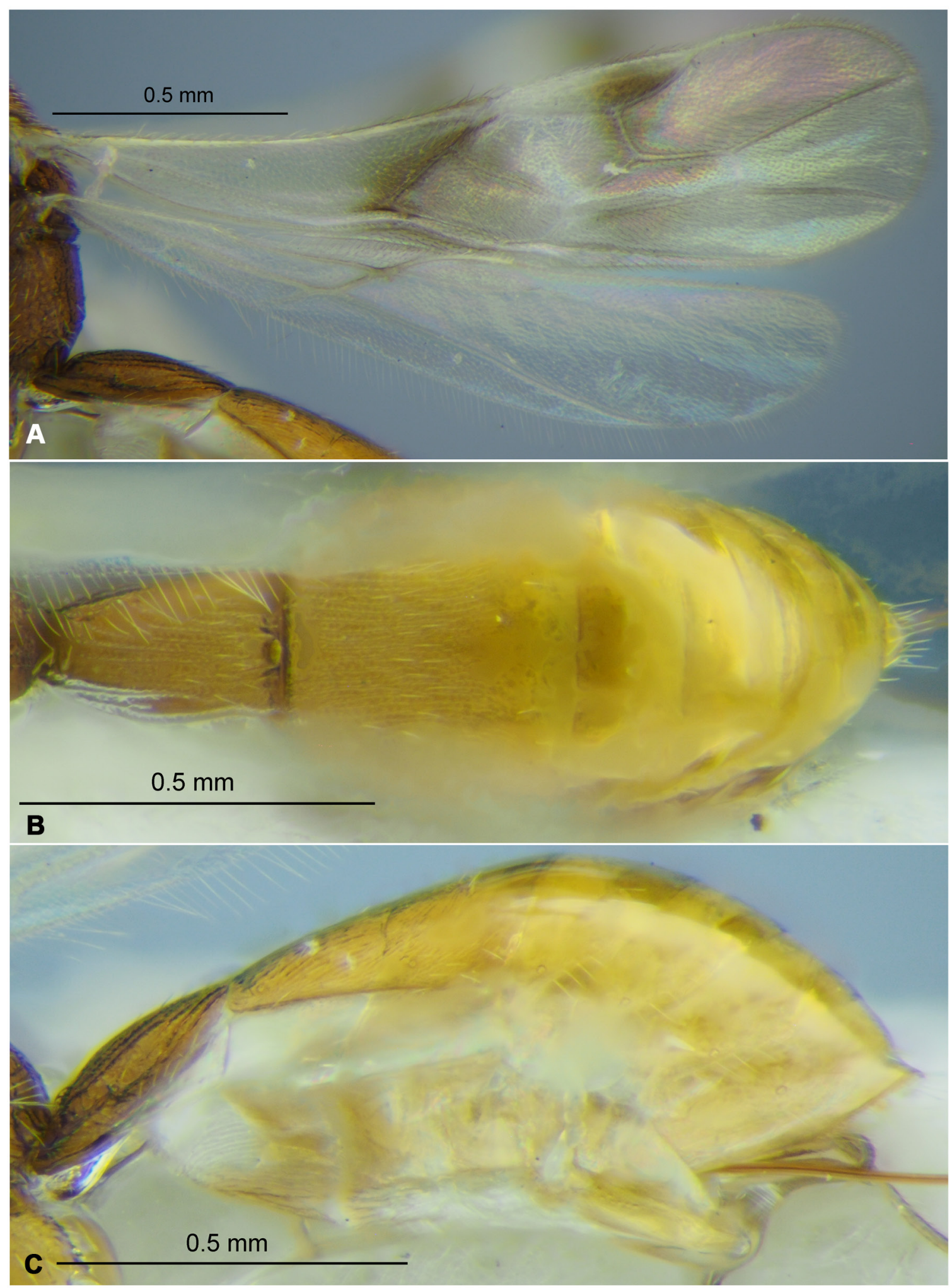

Fig. 4. Dendrosotinus (Gildoria) maculipennis Belokobylskij sp. nov., holotype, $q$ (RMNH). A. Wings. B. Metasoma, dorsal view. C. Metasoma, lateral view. 
times its anterior width, almost equal to length of third tergite. Second suture fine to very fine especially medially, weakly evenly curved. Median length of second and third tergite combined 1.2-1.4 times anterior width of second tergite, 0.9 times their maximum width. Ovipositor sheath $0.5-0.6$ times as long as metasoma, $0.8-1.1$ times as long as mesosoma, $0.4-0.5$ times as long as fore wing.

SCULPTURE AND PUBESCENCE. Vertex entirely and densely irregularly transversely striate with very dense and numerous rugosity; frons densely transversely striate with dense rugosity between striae, striae medially angled down; temple obliquely striate-rugulose in upper two-third, finely rugulose to smooth in lower third; face densely transversely striate, almost smooth medially on narrow area. Mesosoma almost entirely densely transversely striate-rugulose, partly with fine granulation; scutellum entirely densely and finely transversely striate-rugulose. Mesopleuron striate-rugulose in upper half, densely reticulatecoriaceous in lower half. Propodeum without delineated areas, with distinct median longitudinal carina in anterior third, entirely densely areolate-reticulate. Hind coxa rugulose-striate in upper half and densely finely reticulate-coriaceous in lower half; hind femur dorsally and laterally densely rugulosestriate, finely reticulate- coriaceous ventrally. First metasomal tergite entirely longitudinally striate with very dense reticulation between striae, without distinct dorsal carinae. Second tergite entirely and third in anterior third densely striate with dense reticulation, striae laterally curved; third tergite medially densely small reticulate-coriaceous on short area and laterally reticulate-coriaceous on long area; smooth posteriorly. Following tergites entirely smooth. Vertex mainly with sparse short almost appressed pale setae. Mesoscutum entirely with short, very dense yellow setae. Setae of hind tibia semi-erect, short and rather sparse, their length on dorsal surface $0.3-0.4$ times maximum width of tibia.

CoLour. Body mainly light reddish brown, darkened dorsally, rarely body mainly reddish brown and darker dorsally, head below and metasoma behind first or third tergite brownish yellow to almost yellow; rarely body distinctly darkened, reddish brown. Palpi pale yellow. Antenna mainly yellow to brownish yellow or sometimes reddish brown, $7-11^{\text {th }}$ apical segments brown to dark brown or black; rarely (in dark form) antenna mainly reddish brown to black, six anterior segments faintly paler. Legs mainly yellow with brownish tint, rarely brownish yellow; hind coxa and femur pale brown to yellowish brown. Ovipositor sheath brown in anterior half, almost black in posterior half. Fore wing distinctly maculate, with wide infuscate transverse stripes along basal cell, below middle of pterostigma and subdistally, hyaline on remaining large areas. Pterostigma dark brown in apical half, whitish yellow or white in basal half and and apically at short distance.

\title{
Male \\ Unknown.
}

\section{Distribution}

Yemen.

\author{
Dendrosotinus (Gildoria) subelongatus Belokobylskij sp. nov. \\ urn:1sid:zoobank.org:act:09D12F3B-FE94-4ABC-9854-B26E98F9109E
}

Figs 5-6

\section{Comparative diagnosis}

This new species is very similar to D. (G.) elongatus van Achterberg, 2003 (van Achterberg 2003) known only from the Canary Islands, but differs from it by the ovipositor sheath short, distinctly shorter than fore wing (longer than fore wing in D. elongatus), vertex rather evenly and finely curvedly transversely striate-reticulate (coarsely reticulate with undulate striation in D. elongatus), second radial abscissa (3SR) of fore wing short, 0.7 times as long as third abscissa (SR1) (long, almost equal in D. elongatus), hind tarsus long, 0.9 times as long as hind tibia (short, 0.7 times as long as hind tibia in D. elongatus), 
propodeum with delineated areola (without areola in D. elongatus), and body mainly brownish yellow to yellow partly (mainly reddish brown to partly dark reddish brown in D. elongatus).

\section{Etymology}

Name derived from a combination of 'sub' (Latin for 'under') and 'elongatus' (Latin for 'elongate') for its similarity to D. elongatus.

\section{Material examined}

Holotype

UNITED ARAB EMIRATES • +; "United Arab Emirates, Fujairah (1314), light tr., 13-19.iv.2005, $25^{\circ} 08^{\prime} \mathrm{N}, 56^{\circ} 21^{\prime}$ E. A.v. Harten, RMNH'05”; RMNH.

\section{Paratype}

UNITED ARAB EMIRATES • 1 क; "United Arab Emirates, Fujairah (1587), hand coll., 2-13.v.2005, $25^{\circ} 08^{\prime} \mathrm{N}, 56^{\circ} 21^{\prime}$ E. A.v. Harten, RMNH’05”; RMNH.

\section{Description}

\section{Female}

Measurements. Body length 2.0-2.5 mm; fore wing length 1.6-1.9 mm.

HEAD. Head width (dorsal view) 1.4-1.6 times its median length, 1.2 times width of mesoscutum. Head behind eyes (dorsal view) weakly convex in anterior half, weakly roundly narrowed in posterior half. Transverse diameter of eye (dorsal view) 1.2-1.4 times longer than temple. Ocelli small, arranged in triangle with base 1.2-1.3 times as wide as its sides. POL 4.0-4.5 times Od, 0.9-1.0 times OOL. Eye glabrous, its maximum diameter 1.10-1.15 times minimum diameter. Malar space 0.55-0.60 times maximum diameter of eye, 0.8-0.9 times basal width of mandible. Face convex, its width 1.2 times maximum diameter of eye, 1.4-1.5 times height of face and clypeus combined. Clypeal suture almost complete, distinct laterally and fine dorsally. Hypoclypeal depression medium-sized and rounded, its width 0.7 times shortest distance from edge to eye, $0.35-0.40$ times width of face. Occipital carina dorsally complete and not broken toward ocellar triangle, not reaching below hypostomal carina and obliterated at rather long distance before mandible base. Hypostomal flange very narrow.

AntenNa. Antenna rather slender, almost filiform, 18-segmented, 0.8 times as long as body. Scape 1.41.5 times longer than maximum width. First flagellar segment 5.0-6.0 times longer than apical width, about as long as second segment. Penultimate segment 2.5 times longer than width. 0.6 times as long as first segment, about as long as apical segment; latter acuminate apically.

Mesosoma. Mesosoma not depressed, its maximum length 1.9-2.1 times its maximum height. Pronotal carina fine, situated submedially. Mesoscutum (lateral view) not highly, but distinctly curvedly elevated above pronotum, its median lobe (dorsal view) protruding forwards, distinctly convex anteriorly and without anterolateral corners, with almost complete, rather deep posteriorly and very shallow anteriorly median longitudinal furrow; mesoscutum in dorsal view 1.1 times longer than wide. Notauli complete, entirely deep, crenulate-rugulose. Prescutellar depression deep, longer laterally than medially, evenly curved, with 5-7 curved carinae, finely rugulose between carinae, 0.3-0.5 times as long as scutellum. Scutellum distinctly convex, with distinct lateral carinae, 1.0-1.1 times longer than maximum anterior width. Subalar depression rather wide, shallow, rugulose with curved striation. Precoxal sulcus distinct, shallow anteriorly and rather deep posteriorly, rather wide, densely reticulate, running along anterior 0.5-0.6 of lower part of mesopleuron. Metanotum with low, wide and rounded distally medio-dorsal tubercle (lateral view). Metapleural flange (lobe) wide, short and subrounded distally. Propodeum weakly convex, without distinct lateral tubercles. 
WINGS. Fore wing 3.6-3.8 times longer than wide. Pterostigma rather narrow, 5.2-6.5 times longer than its maximum width. Radial vein (r) arising behind middle of pterostigma, from its basal $0.55-0.60$. Radial (marginal) cell not shortened, metacarpus (1-R1) 1.0-1.1 times longer than pterostigma. Second radial abscissa (3-SR) 2.4-2.5 times longer than first abscissa (r) and forming with it weakly obtuse angle, $0.65-0.70$ times as long as the weakly or very weakly curved third abscissa (SR1), 1.3 times longer than

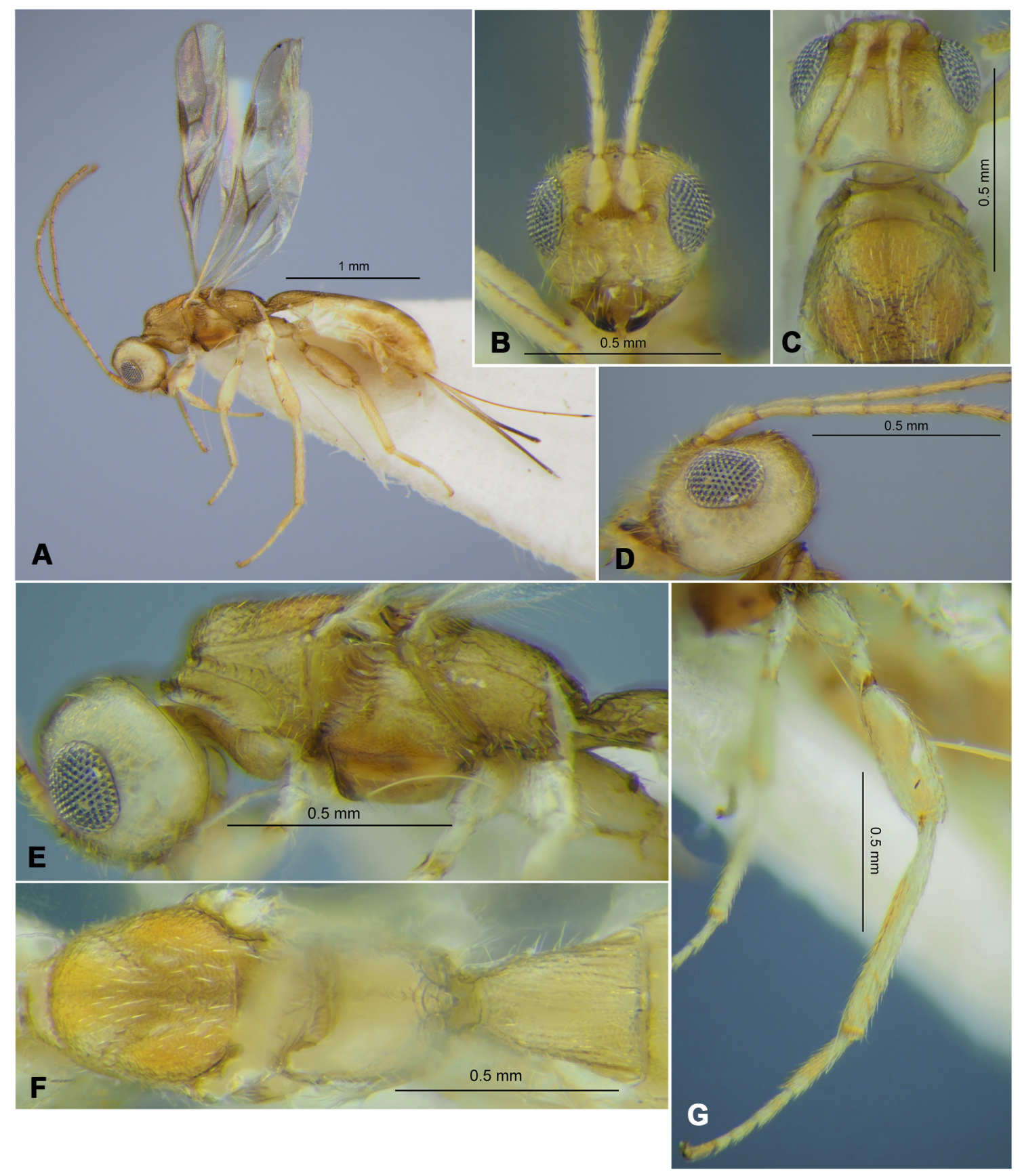

Fig. 5. Dendrosotinus (Gildoria) subelongatus Belokobylskij sp. nov., holotype, $q$ (RMNH). A. Habitus, lateral view. B. Head and basal segments of antenna, front view. C. Head and mesoscutum, dorsal view. D. Head and basal segments of antenna, lateral view. E. Head and mesosoma, lateral view. F. Mesosoma and first metasomal tergite, lateral view. G. Hind leg. 
straight first radiomedial vein (2-SR). Second radiomedial (submarginal) cell not narrowed distally, its length 3.1-3.3 times maximum width, 1.5-2.0 times length of narrow brachial (subdiscal) cell. Second abscissa of medial vein (2-SR+M) rather long, 0.4-0.6 times as long as recurrent vein (m-cu). Third abscissa of medial vein (2-M) very weakly sinuate. Nervulus (cu-a) short, distinct, strongly postfurcal, distance between basal vein (1-M) and nervulus (cu-a) 1.3-1.5 times nervulus (cu-a) length. Mediocubital vein $(\mathrm{M}+\mathrm{CU} 1)$ rather evenly and more or less distinctly curved to longitudinal anal vein (1-1A). Narrow brachial (subdiscal) cell closed apically distinctly before recurrent vein (m-cu), apically reclivous and curved. Parallel vein (CU1a) interstitial. Hind wing 4.6 times longer than its maximum width. First costal abscissa $(\mathrm{C}+\mathrm{SC}+\mathrm{R}) 0.9$ times as long as second abscissa (1-SC+R). First abscissa of mediocubital vein $(\mathrm{M}+\mathrm{CU}) 0.5$ times as long as second and third abscissae (1-M) combined. Recurrent vein (m-cu) short, unsclerotised and not pigmented, distinctly antefurcal, strongly oblique and curved towards base of wing.

Legs. Hind coxa without basoventral tubercle, but with weak convex basoventral corner, 1.7 times longer than maximum width. Hind femur elongate-oval, 3.0-3.1 times longer than wide. Hind tibia without spines on outer distal margin. Hind tarsus $0.9-1.0$ times as long as hind tibia. Hind basitarsus thickened, 0.6 times as long as remaining segments combined. Second segment of hind tarsus $0.55-0.60$ times as long as basitarsus, 1.3-1.4 times longer than narrow fifth segment (without pretarsus).

Metasoma. Metasoma 1.1 times longer than mesosoma and head combined. First segment with weakly elongated acrosternite, its length 0.3 times tergite length. First tergite rather long and not strongly wide, with spiracular protuberances in anterior third, distinctly and almost linearly widened from base to apex. Length of first tergite 1.3-1.4 times its maximum posterior width; posterior width 2.0-2.2 times its minimum anterior width. Second suture fine to very fine laterally, absent medially, with weakly sublateral bands. Second tergite with very shallow, subparallel, sublateral and almost straight narrow depression in anterior half. Median length of second and third tergite combined 1.5-1.6 times anterior width of second tergite, almost equal to their maximum width. Ovipositor sheath $0.9-1.0$ times as long as metasoma, $1.3-1.4$ times as long as mesosoma, $0.70-0.75$ times as long as fore wing.

SCULPTURE AND PUBESCENCE. Vertex entirely and densely reticulate-areolate, usually arranged in curved transverse striation, often with very dense and numerous additional coriaceous; frons densely transversely undulately striate with dense rugosity-reticulation between striae; temple oblique striaterugulose in upper two-third, almost smooth in lower third; face densely transversely striate, almost smooth between striae. Mesoscutum entirely densely rugulose-reticulate arranged in transverse semicurved striae, partly with fine granulation, rugulose in narrow medio-posteriar area; scutellum entirely densely and finely rugulose-reticulate. Mesopleuron mainly densely reticulate-coriaceous. Propodeum with basolateral areas distinctly delineated by carinae, areas entirely rather finely and densely reticulatecoriaceous, median longitudinal carina distinct in anterior half; areola rather large, sparsely rugosestriate and delineated by irregular carinae. Hind coxa mainly rugulose- or rugulose-striate; hind femur distinctly coriaceous almost entirely, rugose-striate in dorsal half. First metasomal tergite entirely densely longitudinally striate with dense reticulation between striae, without distinct dorsal carinae. Second tergite entirely and third in anterior third densely and rather finely, weakly curvedly striate with dense fine reticulation, laterally striation fine or absent; third tergite smooth on remaining part. Following tergites entirely smooth. Vertex mainly with sparse short semi-erect pale setae. Mesoscutum entirely with short, dense semi-erect yellow setae. Setae of hind tibia semi-erect, short and rather dense, their length on dorsal surface $0.3-0.4$ times maximum width of tibia.

CoLour. Body mainly brownish yellow to yellow partly, faintly infuscate dorsally. Palpi pale yellow. Antenna mainly pale brown, five basal segments yellow. Legs mainly almost entirely yellow, whitish yellow basally. Ovipositor sheath almost yellow in anterior half, brown in posterior half. Fore wing faintly maculate, with rather distinct infuscate transverse stripes along basal view and below middle of pterostigma. Pterostigma dark brown in apical two-third, whitish yellow or white in basal third. 

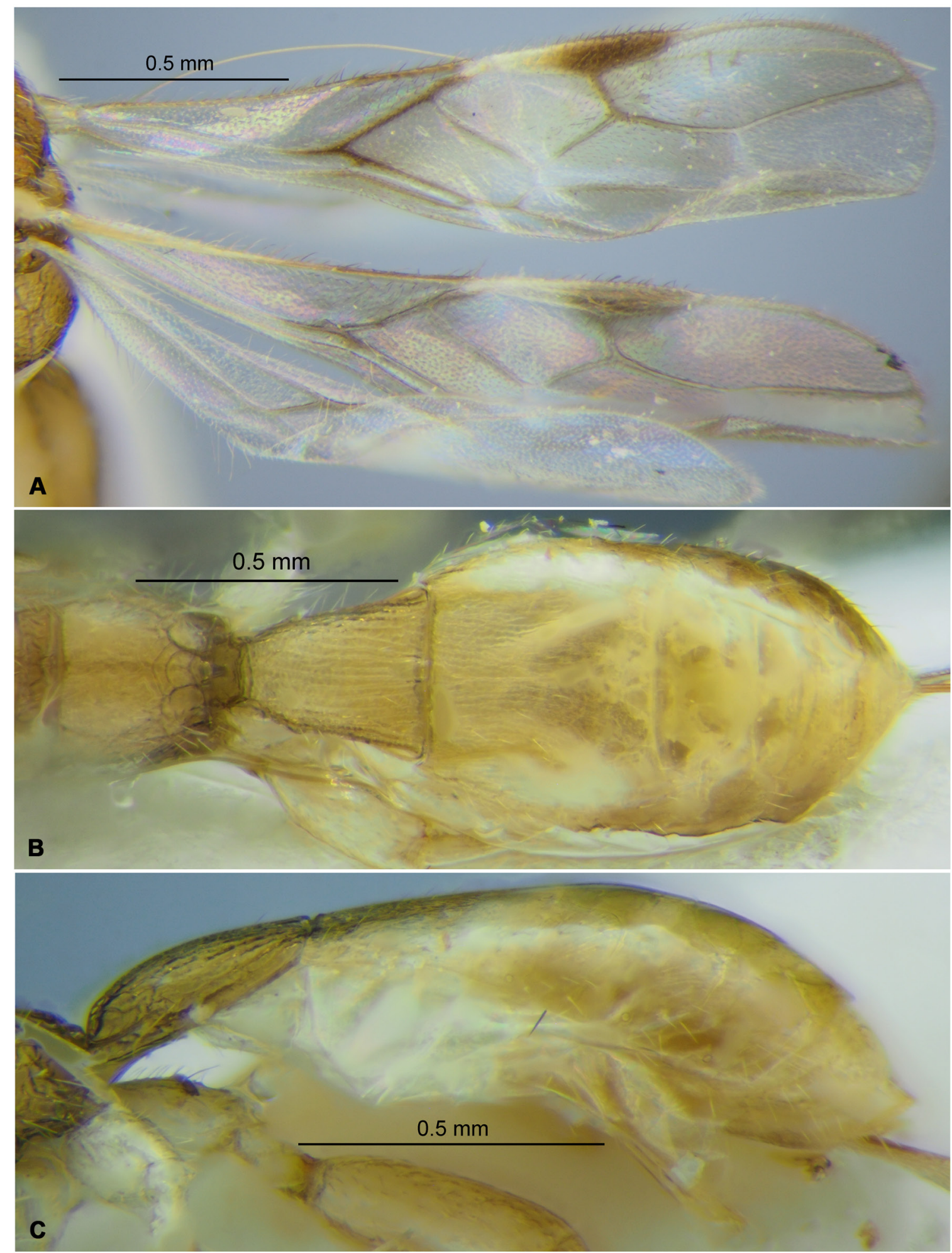

Fig. 6. Dendrosotinus (Gildoria) subelongatus Belokobylskij sp. nov., holotype, $q$ (RMNH). A. Wings. B. Propodeum and metasoma, dorsal view. C. Metasoma, lateral view. 


\section{Male}

Unknown.

\section{Distribution}

Yemen.

Genus Doryctes Haliday, 1836

\section{Type species}

Bracon obliteratus Nees, 1834, designated by Erichson (1837).

\section{Remarks}

Doryctes currently consists of three subgenera, Doryctes s. str., Neodoryctes Szépligeti, 1914 and Plyctes Fischer, 1981. The last two subgenera are sometimes treated as valid genera (Zaldívar-Riverón et al. 2008). The subgenus Neodoryctes is here recorded from the Arabian Peninsula for the first time.

\section{Distribution}

All zoogeographical regions.

Doryctes (Neodoryctes) arrujumi Belokobylskij sp. nov. urn:1sid:zoobank.org:act:7413E4A3-1F4A-4B48-8869-1798B3B67E4A

Figs $7-8$

\section{Comparative diagnosis}

This new species is very similar to Doryctes (Neodoryctes) variegatus Szépligeti, 1914 from Tanzania, but differs from it by sparse setae on the dorsal surface of the hind tibia (dense in D. variegatus), the hind femur mainly brownish yellow or light reddish brown (mainly dark brown in D. variegatus), the median lobe of the mesoscutum with complete shallow medial furrow (without furrow in $D$. variegatus), second radiomedial cell short (longer in $D$. variegatus), and first medial abscissa not or very weakly sinuate (distinctly sinuate in D. variegatus).

\section{Etymology}

Named after the type locality of the new species, Ar Rujum.

\section{Type material}

\section{Holotype}

YEMEN • +; "Yemen (5556), Ar Rujum, 15.i-9.iv.2001, Mal. trap, A.v. Harten RMNH’02”; RMNH.

\section{Paratypes}

YEMEN • 1 क; "Yemen (5700), Ar Rujum, 9.iv-5.vi.2001, Mal. trap; A.v. Harten RMNH’02”; RMNH.

SAUDI ARABIA • 1 q; "Saudi Arabien, W. Büttiker”, "Namas, 2380 m, 17.IV.1980”; BMNH.

\section{Description}

\section{Female}

MEASUREMENTs. Body length 4.8-5.2 mm; fore wing length 3.5-3.9 mm.

HEAD. Head width (dorsal view) 1.4-1.6 times its median length, 1.10-1.15 times width of mesoscutum. Head behind eyes (dorsal view) weakly convex in anterior half, roundly narrowed in posterior half. 
Transverse diameter of eye (dorsal view) 1.3-1.5 times longer than temple. Ocelli medium-sized to small, arranged in triangle with base 1.1 times its sides. POL 1.2-2.0 times Od, 0.5-0.6 times OOL. Eye glabrous, its maximum diameter 1.20-1.25 times minimum diameter. Malar space $0.3-0.4$ times maximum diameter of eye, $0.6-0.8$ times basal width of mandible. Face convex, its width $0.9-1.1$ times maximum diameter of eye, 1.2-1.4 times height of face and clypeus combined. Clypeal suture complete, distinct laterally and dorsally. Hypoclypeal depression medium-sized and rounded, its width 0.9 times shortest distance from edge to eye, 0.4 times width of face. Occipital carina dorsally complete and not broken toward ocellar triangle, not reaching below hypostomal carina and obliterated short distance before mandible base. Hypostomal flange narrow.

AnTENNA. Antenna slender, weakly setiform, 34-segmented, 0.9 times as long as body. Scape 1.6-1.8 times longer than maximum width. First flagellar segment 4.5-5.0 times longer than apical width, 1.1 times longer than second segment. Penultimate segment 4.3 times longer than width. 0.5 times as long as first segment, 0.9 times as long as apical segment; latter acuminate apically.

Mesosoma. Mesosoma not depressed, its maximum length 2.1-2.2 times its maximum height. Pronotal carina distinct, situated in anterior 0.3 of pronotum. Mesoscutum (lateral view) not highly, but distinctly curvedly elevated above pronotum, its median lobe (dorsal view) distinctly protruding forwards, convex anteriorly and without anterolateral corners, with shallow median longitudinal furrow; mesoscutum in dorsal view 1.0-1.1 times as long as maximum width. Notauli complete, rather deep anteriorly and shallow to very shallow posteriorly, rugulose. Prescutellar depression shallow, same length laterally and medially, evenly weakly curved, with 6-7 carinae, smooth between carinae, 0.2 times as long as scutellum. Scutellum weakly convex, with distinct lateral carinae, almost as long as maximum anterior width. Subalar depression rather wide, shallow, striate with reticulation. Precoxal sulcus rather distinct, more or less deep and wide but shallow and narrow in posterior quarter, reticulate-striate, running along anterior $0.8-1.0$ of lower part of mesopleuron. Metanotum without dorsal tubercle (lateral view). Metapleural flange (lobe) wide basally and narrowed distally, short, subpointed distally. Propodeum weakly convex, evenly narrowed posteriorly without distinct lateral tubercles.

Wings. Fore wing 3.5-3.8 times longer than wide. Pterostigma wide, 3.5-4.0 times longer than its maximum width. Radial vein (r) arising almost from middle of pterostigma. Radial (marginal) cell weakly shortened, metacarpus (1-R1) 1.10-1.15 times longer than pterostigma. Second radial abscissa (3-SR) 2.0-2.3 times longer than first abscissa (r) and forming with it obtuse angle, 0.35 times as long as the weakly sinuate third abscissa (SR1), 1.05-1.15 times as long as first radiomedial vein (2-SR). Second radiomedial (submarginal) cell weakly narrowed distally, its length about 2.0 times maximum width, 0.8-0.9 times length of rather wide brachial (subdiscal) cell. Second abscissa of medial vein (2$\mathrm{SR}+\mathrm{M})$ short, $0.2-0.3$ times as long as recurrent vein $(\mathrm{m}-\mathrm{cu})$. First abscissa of medial vein $(1-\mathrm{SR}+\mathrm{M})$ very weakly sinuate; third abscissa (2-M) weakly convex. Nervulus (cu-a) strongly postfurcal, distance between basal vein and nervulus 1.0-1.1 times nervulus length. Mediocubital vein (M+CU1) rather evenly and more or less distinctly curved to longitudinal anal vein (1-1A). Brachial (CU1b) vein strongly declivous, oblique to base of wing. Hind wing 4.5-4.6 times longer than its maximum width. First costal abscissa $(\mathrm{C}+\mathrm{SC}+\mathrm{R}) 0.9$ times as long as second abscissa $(1-\mathrm{SC}+\mathrm{R})$. First abscissa of mediocubital vein $(\mathrm{M}+\mathrm{CU})$ 0.5-0.6 times as long as second and third abscissae (1-M) combined. Recurrent vein (m-cu) long, sclerotised, pigmented, distinctly antefurcal, straight, oblique towards base of wing.

Legs. Hind femur elongate-oval, 2.5-2.7 times longer than wide. Hind tibia with two-three short spines on outer distal margin. Hind tarsus 1.0-1.1 times as long as hind tibia. Hind basitarsus not thickened, $0.7-0.8$ times as long as remaining segments combined. Second segment of hind tarsus $0.45-0.50$ times as long as basitarsus, 1.3 times longer than narrow fifth segment (without pretarsus). 


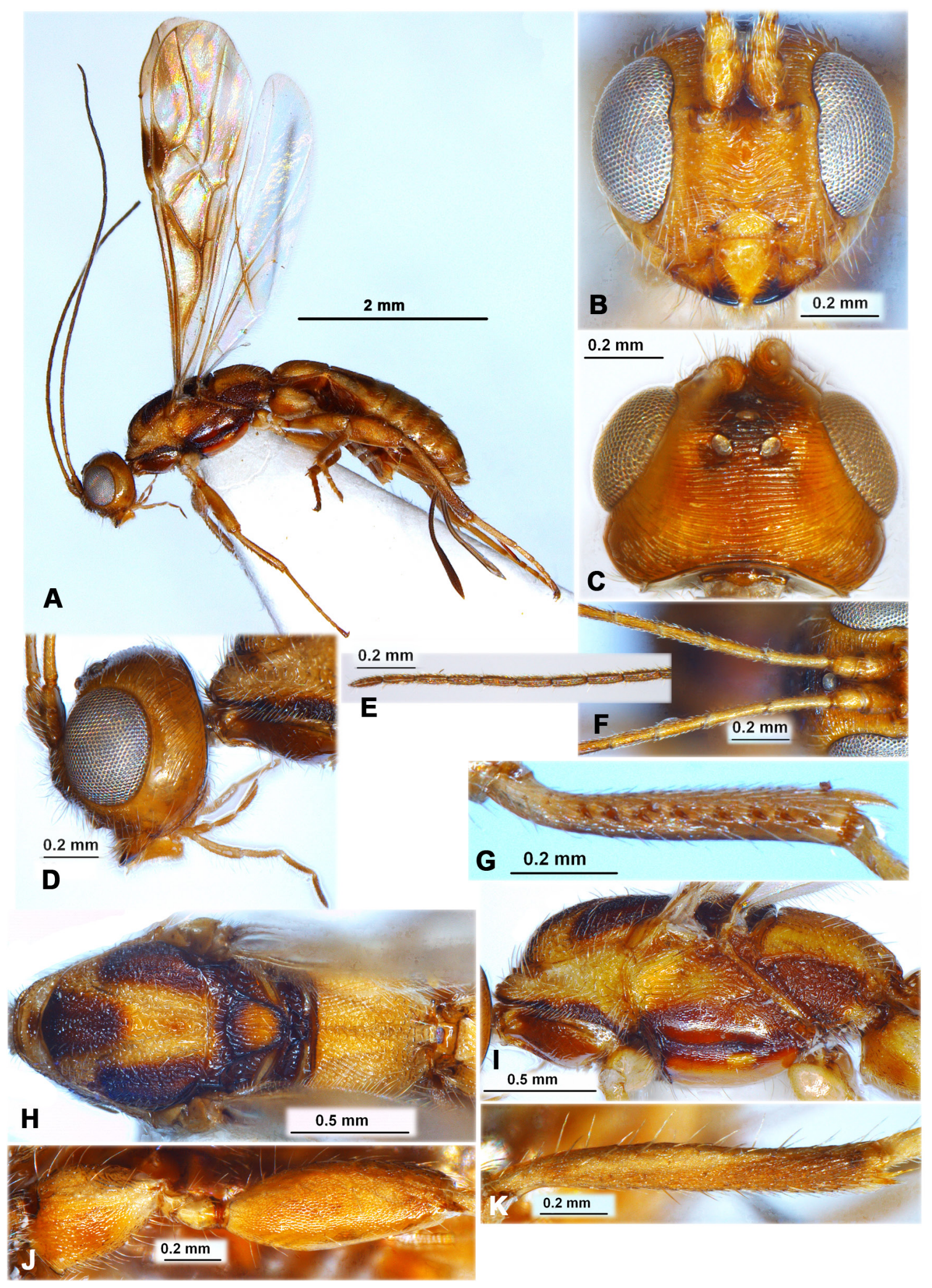

Fig. 7. Doryctes (Neodoryctes) arrujumi Belokobylskij sp. nov., holotype, $q$ (RMNH). A. Habitus, lateral view. B. Head, front view. C. Head, dorsal view. D. Head, lateral view. E. Apical segments of antenna. F. Basal segments of antenna. G. Fore tibia. H. Mesosoma, dorsal view. I. Mesosoma, lateral view. J. Hind coxa and femur. K. Hind tibia. 

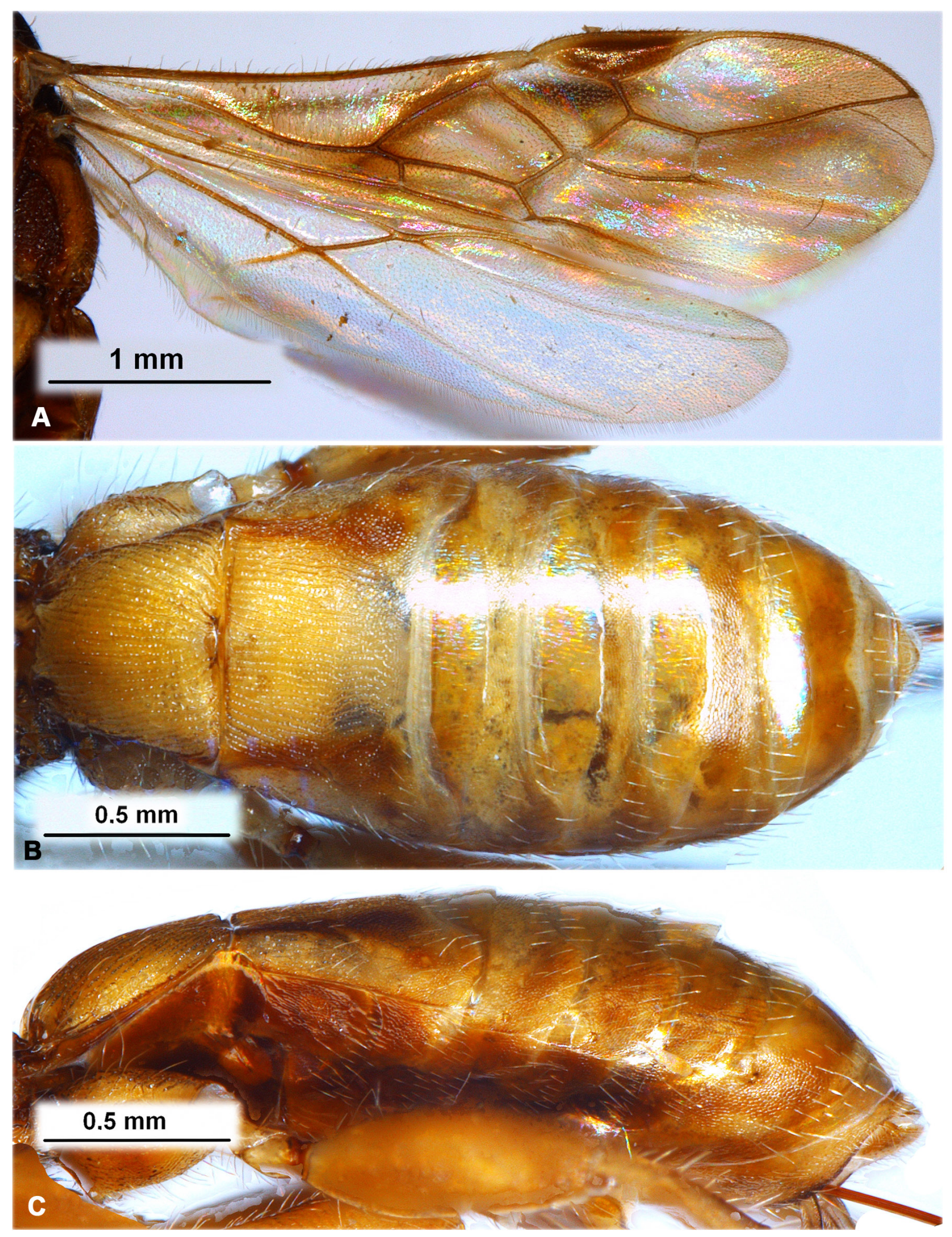

Fig. 8. Doryctes (Neodoryctes) arrujumi Belokobylskij sp. nov., holotype, ${ }^{+}$(RMNH). A. Wings. B. Metasoma, dorsal view. C. Metasoma, lateral view. 
Metasoma. Metasoma 1.0-1.2 times as long mesosoma and head combined. First tergite rather short, without distinct spiracular protuberances, distinctly and almost linearly widened from base to apex. Length of first tergite 0.9-1.1 times its maximum posterior width; posterior width about 2.0 times its minimum anterior width. Second suture fine and narrow, weakly curved medially, with weak sublateral bands. Second tergite with rather fine, subparallel, weakly curved sublateral narrow convex lines. Median length of second tergite 0.45 times anterior width of second tergite, 0.8 times length of third tergite. Ovipositor sheath $0.45-0.50$ times as long as metasoma, $0.6-0.8$ times as long as mesosoma, $0.3-0.4$ times as long as fore wing.

SCULPTURE AND PUBESCENCE. Vertex entirely and densely curvedly striate, without additional sculpture between striae, sometimes (specimen from Saudi Arabia) striae medio-posteriorly distinctly curved and with small circular area medioanteriorly; frons densely transversely or sometimes undulately striate with fine or distinct reticulation or rugosity between striae; face entirely densely curvedly or undulately transversely striate, striae distinctly curved down medially; temple mainly obliquely striate, smooth in lower quarter and sometimes anteriorly near eye. Mesoscutum entirely densely, curvedly, undulately striate with rugulosity, lateral lobes medially densely granulate with fine reticulation or densely obliquely striate; scutellum entirely densely curvedly longitudinally striate with fine or distinct reticulation between striae. Mesopleuron longitudinally striate with reticulation in upper half; medially finely reticulate-coriaceous to smooth, finely and densely reticulate-coriaceous to almost smooth below precoxal sulcus. Propodeum without areas delineated by carinae, entirely densely striate-rugose with reticulation. Hind coxa mainly small and dense rugose-reticulate with punctation, dorsally with few transverse undulate striae; hind femur very densely reticulate-coriaceous. First metasomal tergite entirely densely longitudinally striate with dense reticulation between striae, without distinct dorsal carinae. Second tergite entirely and third in anterior two-thirds densely and distinctly striate with dense fine or distinct reticulation, posteriorly medially and laterally mainly densely reticulate-rugulose to coriaceous, usually without striation; third tergite smooth on remaining part. Fourth to sixth tergites densely and fine reticulate-coriaceous in anterior half or third, smooth on remaining part. Vertex laterally and posteriorly with rather dense or sparse short semi-erect pale setae, glabrous rather widely medially. Mesoscutum with short, dense and semi-erect pale setae arranged widely along notauli and in singly line laterally, glabrous on all lobes medially. Setae of hind tibia semi-erect or sometimes erect, rather short and sparse, their length on dorsal surface 0.6-0.9 times maximum width of tibia.

CoLour. Body mainly brownish yellow to yellow or sometimes and partly light reddish brown, sometimes or always frons, vertex medially, mesoscutum mainly, scutellum, metanotum, propleura, mesopleura in lower half or third and almost all metapleura reddish brown to dark reddish brown; underside of metasoma brown to reddish brown. Antenna mainly dark brown to black, $8-15$ basal segments brownish yellow to brown. Palpi pale yellow basally, sometimes brownish in apical half. Legs mainly yellowish brown, whitish yellow coxae (at least partly) and tarsi; hind tibia pale basally at short or long area. Ovipositor sheath mainly dark brown to black, brown anteriorly. Fore wing widely and faintly maculate. Pterostigma mainly dark brown, whitish yellow or white basally and apically.

\section{Male \\ Unknown.}

\section{Distribution}

Saudi Arabia, Yemen. 
Genus Doryctophasmus Enderlein, 1912

\section{Type species}

Doryctophasmus ferrugineiceps Enderlein, 1912, by monotypy.

\section{Remarks}

This small genus (including only five known species) is already known from the Arabian Peninsula (Belokobylskij 2015).

\section{Distribution}

Afrotropical, Australasian and Oriental regions.

\section{Doryctophasmus ferrugineus (Granger, 1949)}

Doryctes ferrugineus Granger, 1949: 120.

Doryctophasmus ferrugineus - Shenefelt \& Marsh 1976: 1375. — Belokobylskij 2015: 551. — Yu et al. 2016.

\section{Material examined}

UNITED ARAB EMIRATES • 3 우, 1 ภ; " "United Arab Emirates, Sharjah Desert Park (1117), light tr.,

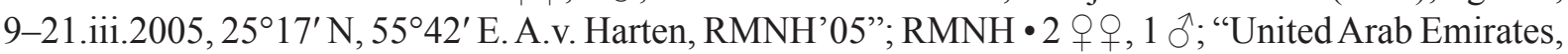

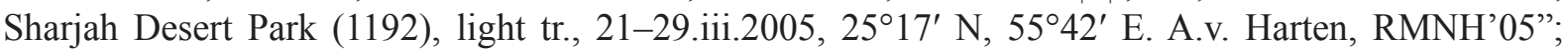
RMNH • 1 q; "United Arab Emirates, Sharjah Desert Park (1202), light tr., 29.iii-6.iv.2005, $25^{\circ} 17^{\prime}$ N, 5542' E. A.v. Harten, RMNH'06”; RMNH • 4 우; “United Arab Emirates, Sharjah Desert Park (1308),

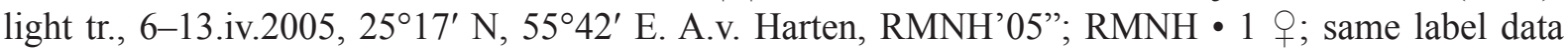
as for preceding; ZISP • 4 우; “United Arab Emirates, Sharjah Desert Park (1394), light tr., 13-23. iv.2005, $25^{\circ} 17^{\prime} \mathrm{N}, 55^{\circ} 42^{\prime}$ E. A.v. Harten, RMNH'05”; RMNH • 1 đo'; same label data as for preceding; ZISP • 2 O O+; “United Arab Emirates, Sharjah Desert Park (1476), light tr., 23-30.iv.2005, $25^{\circ} 17^{\prime}$ N,

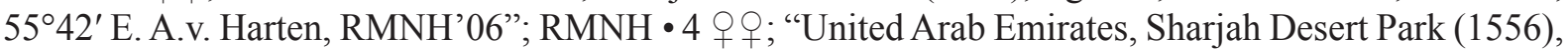

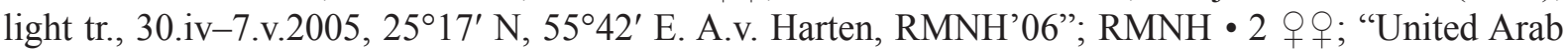

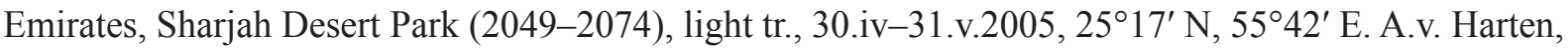
RMNH'05"; RMNH • 7 우, $1 \delta^{\lambda}$; “United Arab Emirates, Sharjah Desert Park (2517), light trap, 20.x.8.xi.2005, $25^{\circ} 17^{\prime} \mathrm{N}, 55^{\circ} 42^{\prime}$ E. A.v. Harten, RMNH'06”; RMNH • 1 '`; same label data as for preceding; ZISP • 2 q 9 ; "United Arab Emirates, Sharjah Desert Park (3692-3697), light tr., 11.xii.2005-18.i.2006, $25^{\circ} 17^{\prime}$ N, 55 42' E. A.v. Harten, RMNH'06”; RMNH • 3 우; “United Arab Emirates, Sharjah Desert

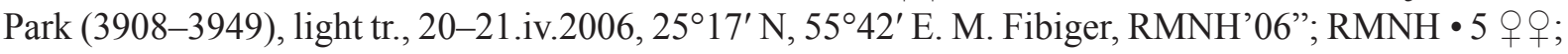

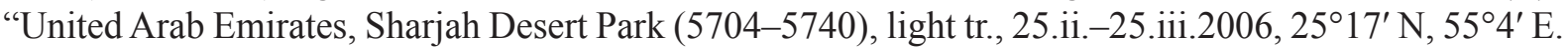

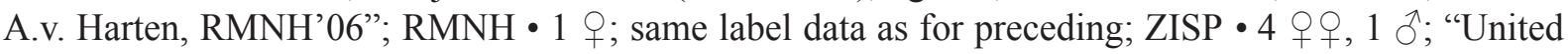
Arab Emirates, Sharjah Desert Park (6491), light trap, 1-25.ii.2006, $25^{\circ} 17^{\prime} \mathrm{N}, 55^{\circ} 42^{\prime}$ E. A.v. Harten, RMNH'06"; 1 क; same label data as for preceding; ZISP • 1 \%; "United Arab Emirates, Sharhah x Khor Kalba (6327-6337), light trap, 24-30.vi.2006, A.v. Harten, RMNH'06”; RMNH • 1 \&; "United

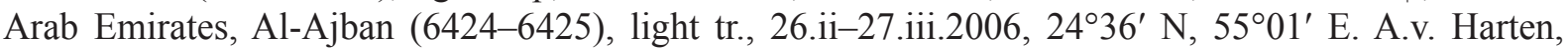

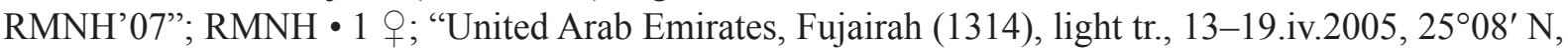
$56^{\circ} 21^{\prime}$ E. A.v. Harten, RMNH'05"; RMNH • 1 ð’; "United Arab Emirates, Sardjah (1700-1706), light trap, 17.iv- 5.vi.2005, $25^{\circ} 21^{\prime}$ N, 55²4' E. A.v. Harten, RMNH'05”; RMNH.

YEMEN • 1 q; "Yemen (6090), Al Kadan, x.2001, light trap, A.v. Harten, T. Abdul-Haq. RMNH'02"; RMNH • 1 O; "Yemen (4054), Al Kowd, viii.1999, light-trap, A.v. Harten \& S. Al Haruri. RMNH'00"; RMNH • 1 \%; same label data as for preceding; ZISP • 1 \%; "Yemen, Al Kowd, ii.2000, light tr., no. 4431 
A.v. Harten \& S. Al Haruri. RMNH'01"; RMNH • 1 q; "Yemen, Al Kowd, v-vi.2000, light tr., no. 4719, A.v. Harten \& S. Al Haruri. RMNH'01"; RMNH • 1 क; "Yemen, Al Kowd, vii.2000, light tr., no. 4820 A.v. Harten \& S. Al Haruri. RMNH'01"; RMNH • 1 q; "Yemen, Al Kowd, i.2001, light tr., no 5482, A.v. Harten \& S. Al Haruri. RMNH'02"; RMNH • 2 우; “Yemen (5750), Al Kowd, 8-12.vii.2001, light trap, A.v. Harten \& S. Al Haruri. RMNH'02"; RMNH • 1 q; same label data as for preceding; ZISP - 1 क; "Yemen (5962), 12 km NW Manakhah, Mal. trap; 3.vii-21.viii.2001, light-trap, A.v. Harten, RMNH'02"; RMNH.

\section{Distribution}

Djibouti, Madagascar, Senegal, the UAE, Yemen (Belokobylskij 2015).

Genus Hemispathius Belokobylskij \& Quicke, 2000

\section{Type species}

Hemispathius polystenoides Belokobylskij \& Quicke, 2000, by original designation.

\section{Remarks}

This genus was originally described from Central Africa (Uganda). Examination of type material for Doryctes species described by Granger (1949) from Madagascar showed that D. pilosus Granger, 1949 actually belongs to Hemispathius (comb. nov.) due to its shorter and wider first metasomal tergite and its shorter acrosternite. This species, characterised by the entirely yellow colouration, is also found in Yemen.

\section{Distribution}

Afrotropical region.

Hemispathius pilosus (Granger, 1949) comb. nov.

Figs 9-10

Doryctes pilosus Granger, 1949: 119.

Doryctes pilosus — Yu et al. 2016.

\section{Material examined}

Lectotype (here designated)

MADAGASCAR • 1 q (missing right fore wing); "EY6197", "Madagascar, Bekily, reg. sud d'ile", "Museum Paris, IV.37, A. Seyrig” "Type”, "Doryctes ferrugineus Granger, 1949 “; MNHN.

\section{Paralectotypes}

MADAGASCAR • 2 우; "EY31767”, "EY31768", "Madagascar, Bekily, reg. sud d'ile", "Museum Paris, IX.36, A. Seyrig"; MNHN • 2 ổ; "EY31769", "EY31770", same label data but "VII.36" and "IX.36"; MNHN.

\section{Additional material}

YEMEN • 2 우 (one without head); "Yemen (5404), Hammam 'Ali, from coffee-berries (with Ceratitis capitata ?), 14.iii.2001, A.v. Harten, RMNH'02"; RMNH.

\section{Distribution}

Madagascar, *Yemen. 


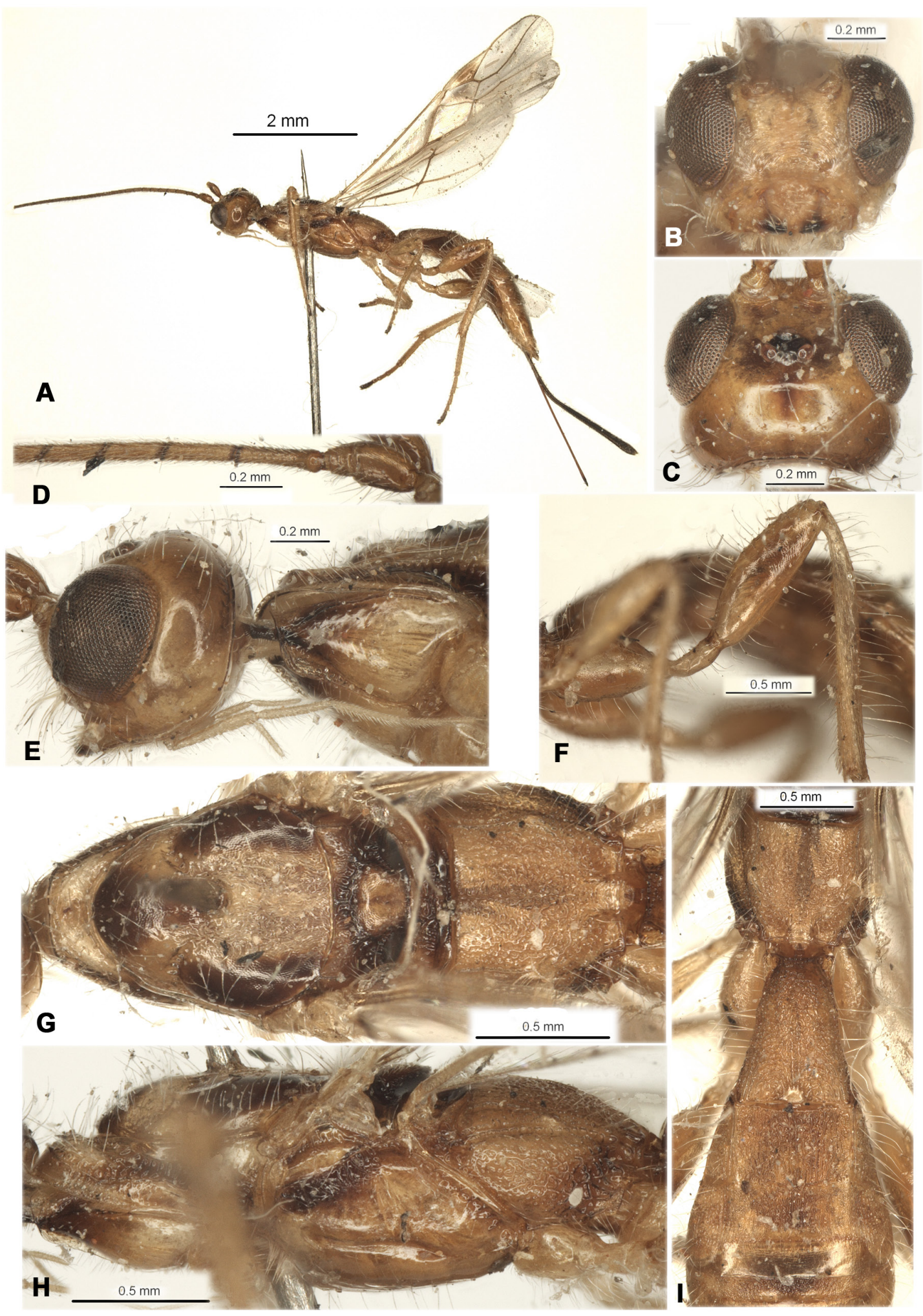

Fig. 9. Hemispathius pilosus (Granger, 1949) comb. nov., paralectotype, $q$ (MNHN). A. Habitus, lateral view. B. Head, front view. C. Head, dorsal view. D. Basal segments of antenna. E. Head, lateral view. F. Hind leg. G. Mesosoma, dorsal view. H. Mesosoma, lateral view. I. Propodeum and three basal tergites of metasoma, dorsal view. 

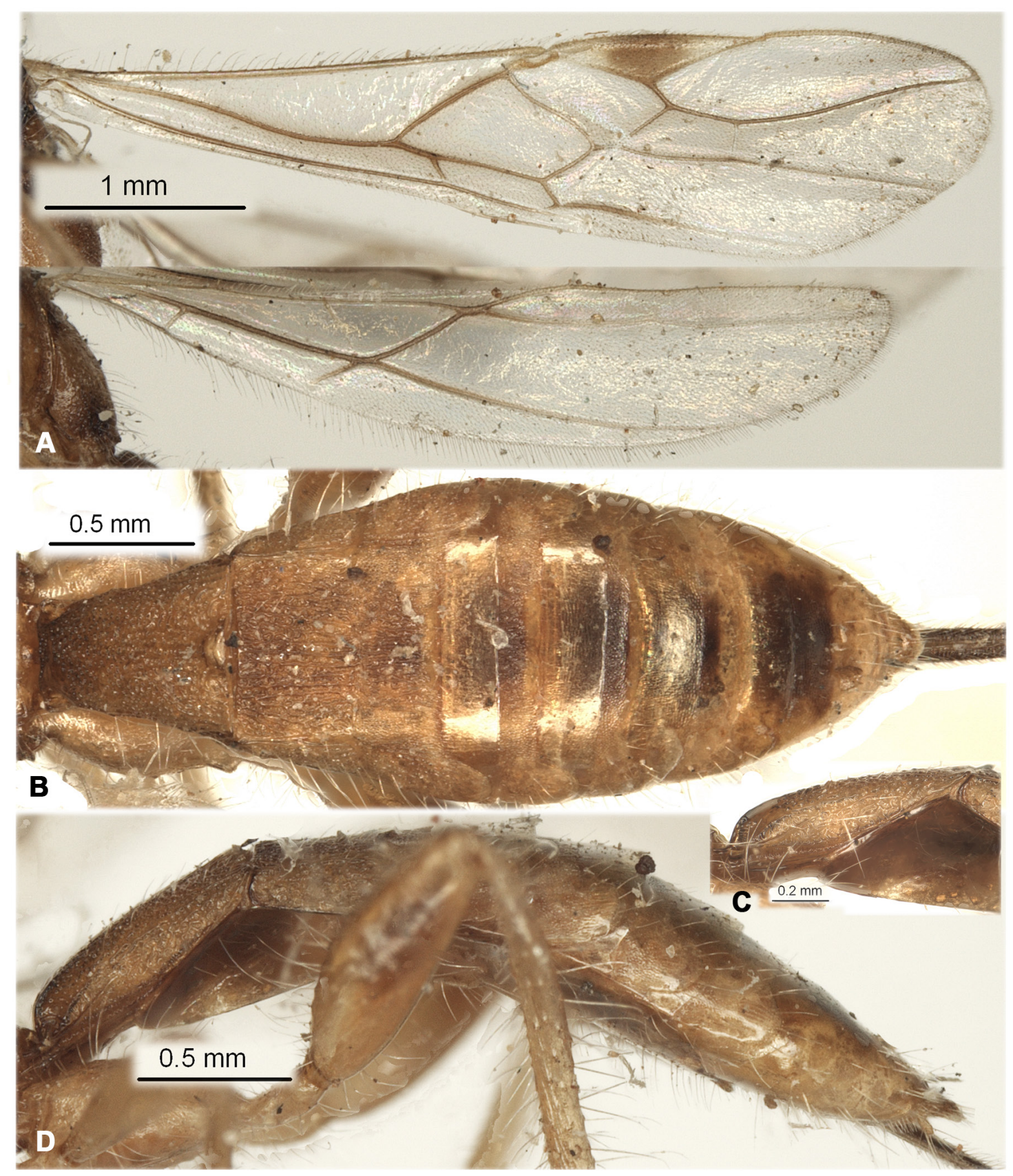

Fig. 10. Hemispathius pilosus (Granger, 1949) comb. nov., paralectotype, $q$ (MNHN). A. Wings. B. Metasoma, dorsal view. C. First metasomal segment, lateral view. D. Metasoma, lateral view. 
Genus Mimodoryctes Belokobylskij, 2001

\section{Type species}

Mimodoryctes proprius Belokobylskij, 2001, by original designation.

\section{Remarks}

This small genus conteins two described species from the deserts of North Africa and of the Arabian Peninsula.

\section{Distribution}

Afrotropical and South Palaearctic regions.

Mimodoryctes arabicus Edmardash, Gadallah \& Soliman, 2020

Fig. 11

Mimodoryctes arabicus Edmardash, Gadallah \& Soliman, 2020: 61.

\section{Material examined}

YEMEN • 2 우; "Yemen (5404), Hammam 'Ali, from coffee-berries (with Ceratitis capitata ?), 14.iii.2001, A.v. Harten, RMNH'02"; RMNH • 1 o ; same label data as for preceding; ZISP • 1 o ; "Yemen (4054), Al Kowd, viii.1999, light-trap, A.v. Harten \& S. Al Haruri, RMNH'00"; RMNH • 1 O; "Yemen (4055), Al Kowd, ix.1999, light trap, A.v. Harten \& S. Al Haruri. RMNH'00"; RMNH • 1 ㅇ, 1 §`’; "Yemen (5750), Al Kowd, 8-12.vii.2001, light trap, A.v. Harten \& S. Al Haruri. RMNH'02"; RMNH • 2 우, 1 ○े; "Yemen (8136), Al Kowd, ix.2003, light trap, A.v. Harten, S. Al Haruri, RMNH’03”; RMNH.

\section{Distribution}

Saudi Arabia, *Yemen.

\section{Remarks}

The status of this taxon is still under discussion because the only character that separates it from M. proprius is the presence of vein r-m of the fore wing (Edmardash et al. 2020). The holotype of M. proprius from Algeria as well as two females from Saudi Arabia and Yemen lack the second radiomedial vein $(\mathrm{r}-\mathrm{m})$ in fore wing combined with an evenly curved second and third radial abscissae (3-SR+SR1); this is considered to be an original character state of Mimodoryctes. However, 10 additional specimens (eight females and two males) from Yemen have the second radiomedial vein ( $\mathrm{r}$ $\mathrm{m}$ ) distinctly developed separating a comparatively small second radiomedial (submarginal) cell and are here listed as M. arabicus. All other morphological features of these specimens indicate that they belong to $M$. proprius. Variation (presence/absence) of the second radiomedial vein (r-m) is known to occur in some species of the Hecabolini genera Leluthia and Pareucorystes, but such variation has never been recorded before in members of the tribe Doryctini. Therefore, additional material as well as additional data (preferably molecular) are needed to solve this question.

Mimodoryctes proprius Belokobylskij, 2001

Figs 12-13

Mimodoryctes proprius Belokobylskij, 2001a: 453.

Mimodoryctes proprius - Yu et al. 2016. — Edmardash et al. 2020: 66. 

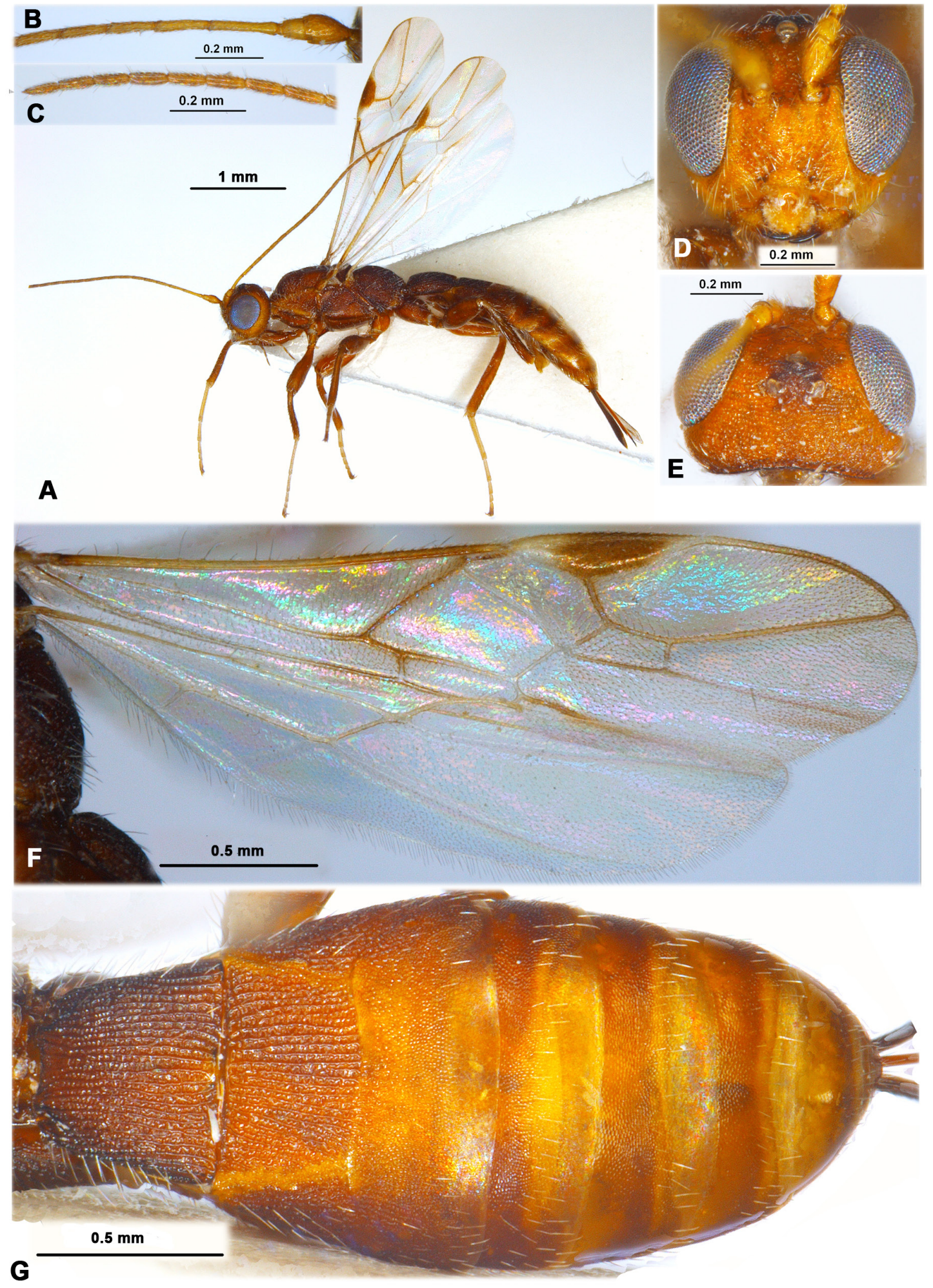

Fig. 11. Mimodoryctes arabicus Edmardash, Gadallah \& Soliman, 2020, $q$ (RMNH). A. Habitus, lateral view. B. Basal segments of antenna. C. Apical segments of antenna. D. Head, front view. E. Head, dorsal view. F. Wings. G. Metasoma, dorsal view. 


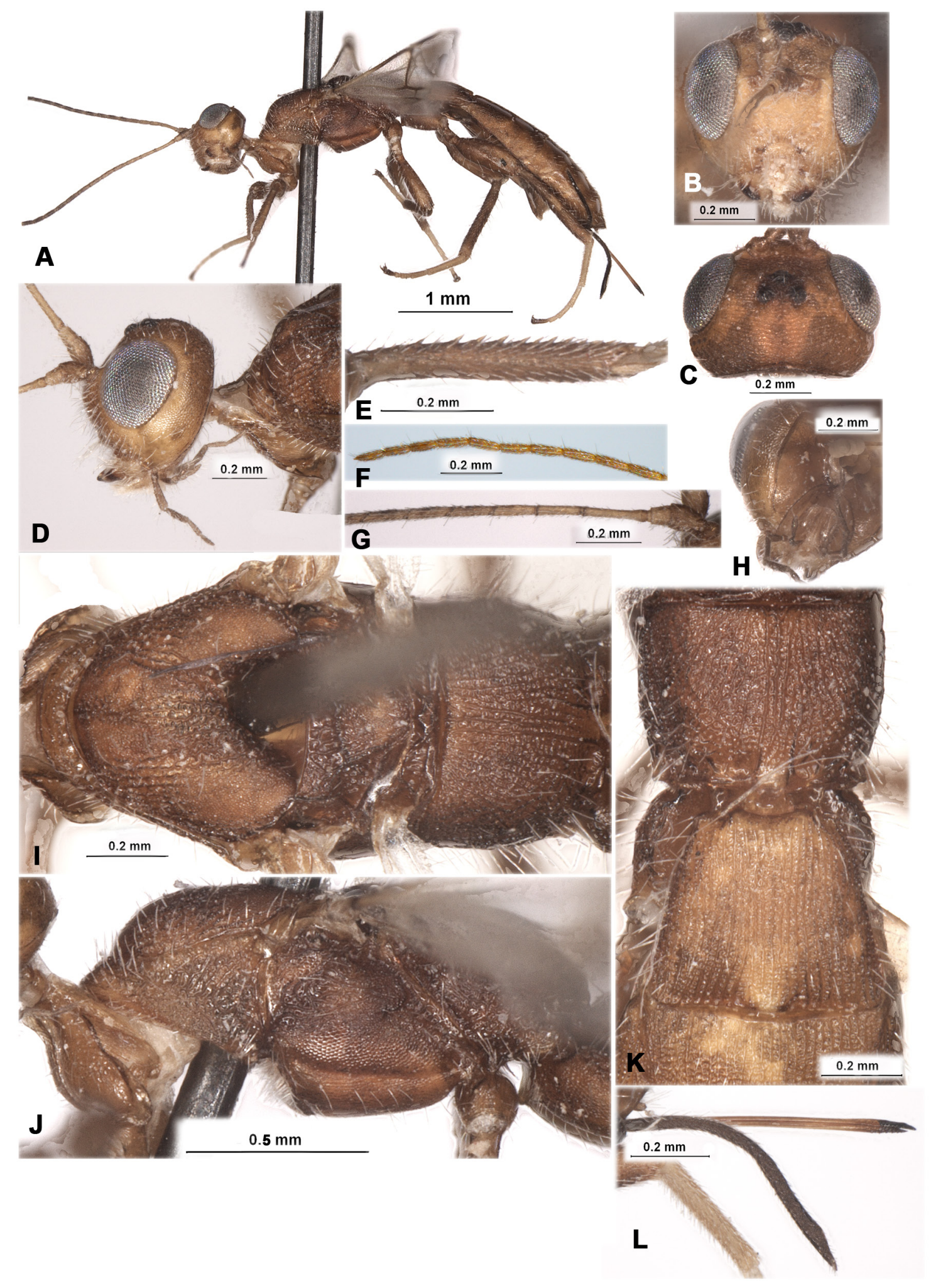

Fig. 12. Mimodoryctes proprius Belokobylskij, 2001, holotype, $q$ (MNBG). A. Habitus, lateral view. B. Head, front view. C. Head, dorsal view. D. Head, lateral view. E. Fore tibia. F. Apical segments of antenna. G. Basal segments of antenna. H. Head, latero-posterior view. I. Mesosoma, dorsal view. J. Mesosoma, lateral view. K. Propodeum and first tergite of metasoma, dorsal view. L. Ovipositor. 

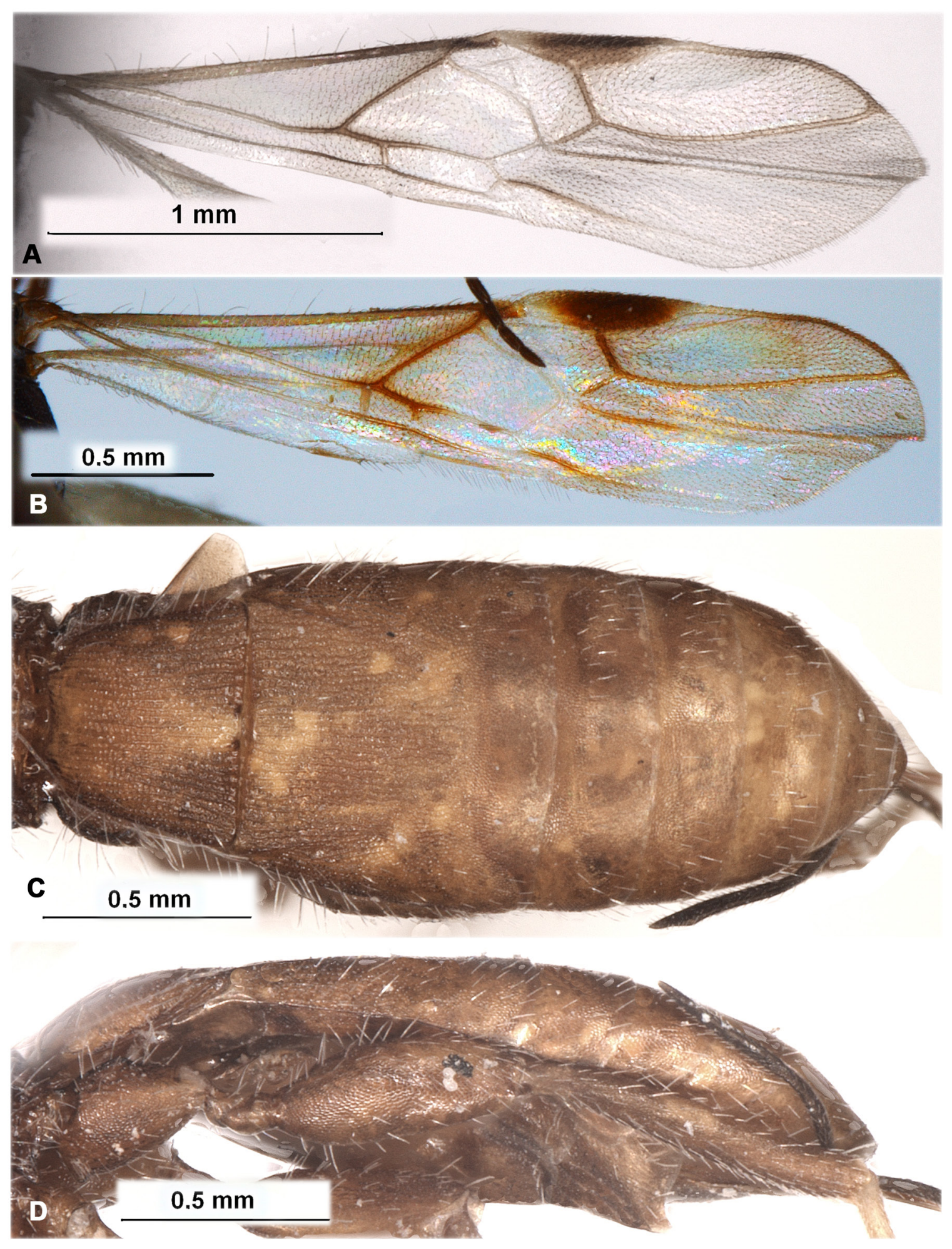

Fig. 13. Mimodoryctes proprius Belokobylskij, 2001. A, C-D. Holotype, $q$ (MNBG). B. Arabian specimen (RMNH). A. Fore wing. B. Fore and hind wings. C. Metasoma, dorsal view. D. Metasoma, lateral view. 


\section{Material examined}

YEMEN • 1 क; "Yemen (6141), Al Kowd, 16-20.viii.2001, light trap, A.v. Harten \& S. Al Haruri. RMNH'02"; RMNH.

\section{Distribution}

Algeria, Saudi Arabia, *Yemen.

Tribe Spathiini Marshall, 1872

Genus Parana Nixon, 1941

\section{Type species}

Parana clotho Nixon, 1943, by monotypy.

\section{Remarks}

This rare genus was originally described from India (Nixon 1943), and it is only known from its type species. A second species of Parana is described here from the United Arab Emirates and Yemen.

\section{Distribution}

Afrotropical and Oriental regions.

Parana arabica Belokobylskij sp. nov. urn:1sid:zoobank.org:act:A3822C53-EC81-401C-A10A-EA53A5544086

Figs $14-15$

\section{Comparative diagnosis}

This new species differs from the type species of the genus by the following characters: furrow between second and third tergites present but shallow (without furrow in P. clotho), face entirely densely reticulateareolate (mainly smooth in P. clotho), antennal segments slender and longer (short and thick in P. clotho), mesoscutum and mesopleuron distinctly reticulate areolate (very finely coriaceous in $P$. clotho), neck long in dorsal view (short in P. clotho), propodeum and petiole long (shorter in P. clotho), radial vein arising behind middle of pterostigma (weakly before middle in $P$. clotho), hyaline stripes on fore wing wide (narrow in $P$. clotho).

\section{Etymology}

Named after its origin, the Arabian Peninsula.

\section{Material examined}

\section{Holotype}

UNITED ARAB EMIRATES - + ; "United Arab Emirates, SSW of ad-Dhaid (6154), light tr., 2430.v.2006, $25^{\circ} 09^{\prime}$ N, 5548' E, A.v. Harten, RNMH’06”; RMNH.

\section{Paratypes}

UNITED ARAB EMIRATES • 2 우; same label as for holotype; RMNH 1 i ; same label data as for preceding; ZISP • 9 우; “United Arab Emirates, Sharjah Desert Park (2049-2074), light tr.,

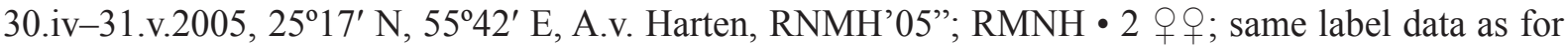
preceding; ZISP • 2 우우 “United Arab Emirates, Sharjah Desert park (2517), light trap, 20.x-8.xi.2005, $25^{\circ} 17^{\prime}$ N, 55 42' E, A.v. Harten, RNMH'06”; RMNH • 1 क; "United Arab Emirates, Sharjah Desert Park

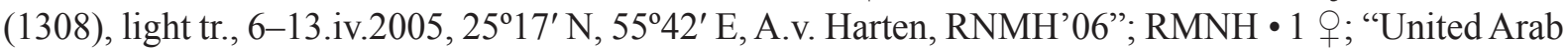




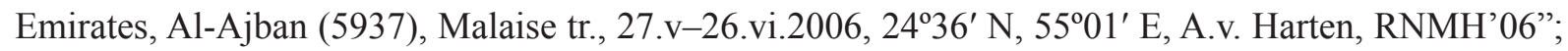

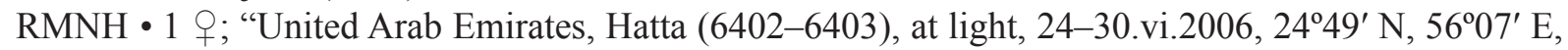
A.v. Harten, RNMH'06"; RMNH - 1 क; "United Arab Emirates, Wadi Safad (5020), at light, 14-

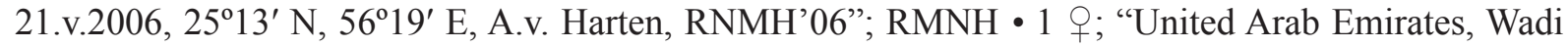

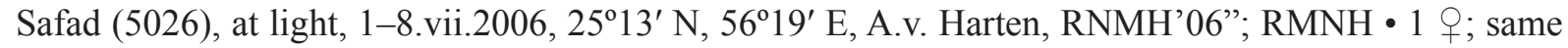
label data as for preceding; ZISP • 2 우; "United Arab Emirates, Wadi Safad (5029), at light, 17-24. vi.2006, $25^{\circ} 13^{\prime}$ N, 56 $19^{\circ}$ E, A.v. Harten, RNMH'06"; RMNH • 1 o ; "United Arab Emirates, Wadi

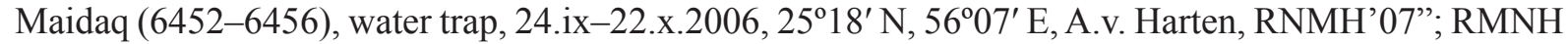
- 1 O; same label data as for preceding; ZISP • 1 क; "Yemen (4055), Al Kowd, ix.1999, light trap, A.v. Harten \& S. Al Haruri. RMNH'00"; RMNH.

\section{Description}

\section{Female}

Measurements. Body length 3.2-4.5 mm; fore wing length $1.7-2.6 \mathrm{~mm}$.

HeAD. Head width (dorsal view) 1.2-1.3 times its median length, 1.2-1.3 times width of mesoscutum. Vertex weakly convex. Head behind eyes (dorsal view) rather distinctly and evenly roundness decreased. Transverse diameter of eye (dorsal view) 1.3-1.4 times longer than temple. Ocelli small, with ocellar triangle base 1.2-1.3 times its sides. POL 2.3-3.2 times Od, 0.6 times OOL. Eye glabrous, in lateral view oblique, its maximum diameter 1.2 times minimum diameter. Malar space $0.6-0.7$ times maximum diameter of eye, 1.3-1.5 times basal width of mandible. Face convex, its width 1.15-1.20 times maximum diameter of eye, 1.3-1.4 times height of face and clypeus combined. Clypeal suture complete, distinct laterally and fine dorsally. Ventral margin of clypeus with distinct flange. Hypoclypeal depression medium-sized and rounded, its width 0.5 times shortest distance from edge to eye, $0.35-0.40$ times width of face. Occipital carina dorsally complete and not broken toward ocellar triangle, not reaching below hypostomal carina and obliterated short distance before mandible base. Hypostomal flange very narrow.

Antenna. Antenna slender, almost filiform, 23-30-segmented, 0.8-0.9 times as long as body. Scape 1.6-1.8 times longer than maximum width. First flagellar segment 5.5-6.0 times longer than apical width, 1.1-1.2 times longer than second segment. Penultimate segment 2.7-3.0 times longer than width, $0.45-0.50$ times as long as first segment, 0.8 times as long as apical segment; latter acuminate apically and without spine.

Mesosoma. Mesosoma not depressed, its maximum length 2.1-2.3 times its maximum height. Pronotal carina rather distinct, its anterior branch situated in anterior $0.40-0.45$, its fine posterior branch laterally not connected with anterior branch, dorsomedially almost fused with posterior margin of pronotum on short distance. Pronotal lateral depression indistinct and not delineated by carinae. Mesoscutum (lateral view) not high, obliquely and curvedly elevated above pronotum, its median lobe (dorsal view) distinctly convex anteriorly and without anterolateral corners, with distinct crenulate longitudinal median furrow in posterior 0.3; mesoscutum in dorsal view 1.20-1.25 times as long as wide. Notauli present only in anterior third (very rarely keeping fine carinae till median furrow) narrow, shallow, crenulate, completely fused posteriorly. Prescutellar depression shallow, rather short medially but widened towards sides, with distinct median carina, finely rugulose-carinate, 0.25 times as long as scutellum. Scutellum slightly convex, with distinct lateral carinae, 1.1-1.2 times as long as maximum anterior width. Subalar depression rather narrow, very shallow, reticulate-rugose. Precoxal sulcus almost indistinct to very shallow and short. Postpectal carina absent. Metanotum with low, wide and distally rounded medio-dorsal tubercle (lateral view). Metapleural flange (lobe) very narrow, short, subpointed, sometimes almost indistinct. Propodeum flat, without lateral tubercles. 


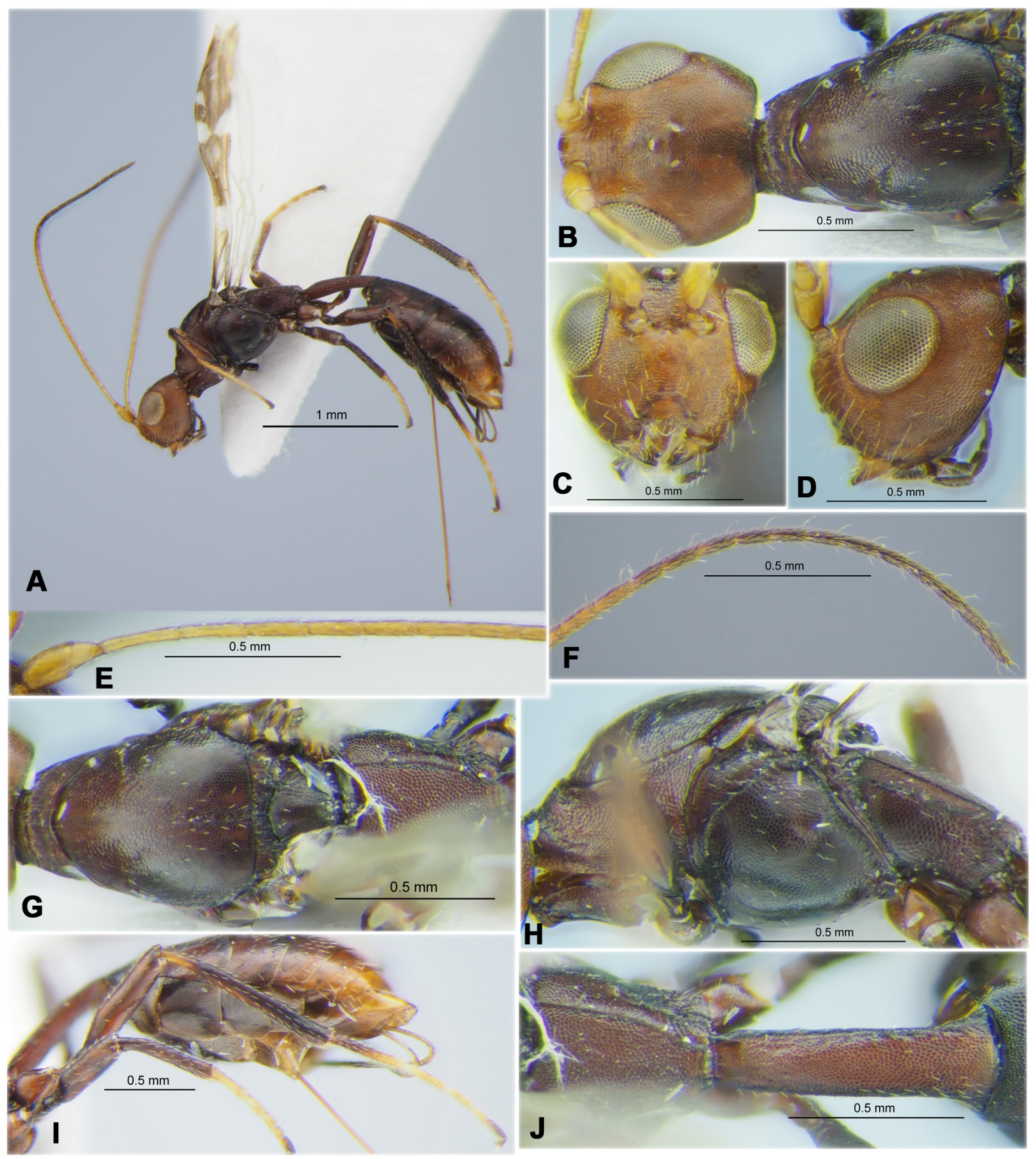

Fig. 14. Parana arabica Belokobylskij sp. nov., holotype, $q$ (RMNH). A. Habitus, lateral view. B. Head and mesoscutum, dorsal view. C. Head, front view. D. Head, lateral view. E. Basal segments of antenna. F. Apical segments of antenna. G. Mesosoma, dorsal view. H. Mesosoma, lateral view. I. Hind leg. J. Propodeum and petiole of metasoma, dorsal view. 

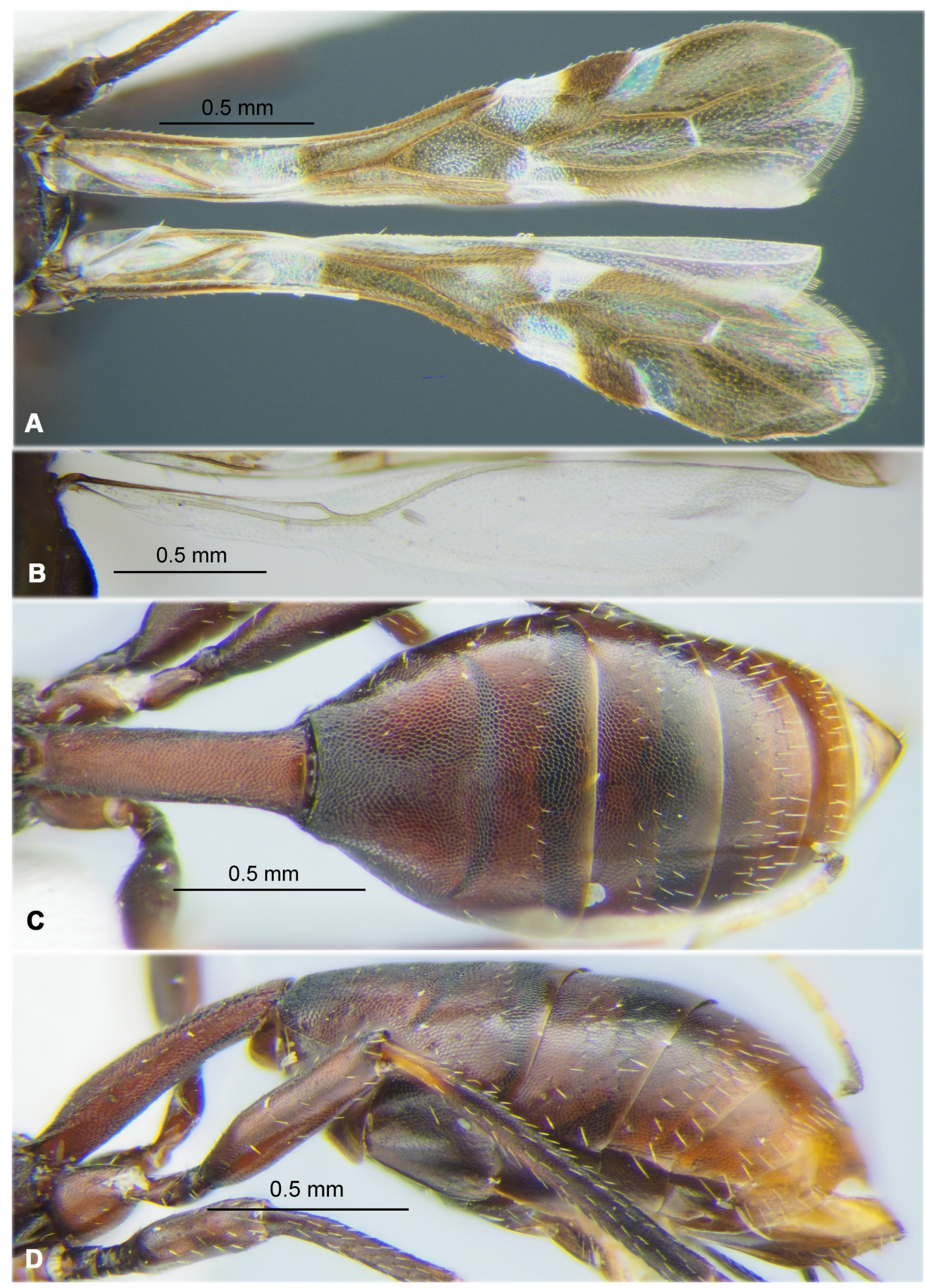

Fig. 15. Parana arabica Belokobylskij sp. nov., holotype, $q$ (RMNH). A. Fore wings. B. Hind wing. C. Metasoma, dorsal view. D. Metasoma, lateral view. 
Wings. Fore wing 4.8-5.4 times longer than wide. Pterostigma narrow in basal half and widened in apical half, 4.2-4.8 times longer than its maximum width. Radial vein (r) arising behind middle of pterostigma, from basal 0.6. Radial (marginal) cell not shortened, metacarpus (1-R1) 1.3-1.4 times longer than pterostigma. Second radial abscissa (3-SR) 2.3-3.3 times longer than first abscissa (r) and forming with it obtuse angle, $0.50-0.65$ times as long as the weakly curved third abscissa (SR1), 1.0-1.3 times as long as weakly curved or straight first radiomedial vein (2-SR). Second radiomedial (submarginal) cell weakly narrowed distally, its length 3.1-3.6 times maximum width, 1.0-1.1 times length of brachial (subdiscal) cell. Second abscissa of medial vein $(2-\mathrm{SR}+\mathrm{M})$ short, $0.8-1.5$ times as long as recurrent vein $(\mathrm{m}-\mathrm{cu})$. Nervulus (cu-a) strongly antefurcal, distance between basal vein and nervulus 1.8-2.5 times nervulus length. Mediocubital vein $(\mathrm{M}+\mathrm{CU} 1)$ submedially strongly sinuate and deep curved to longitudinal anal vein (1-1A). Narrow brachial (subdiscal) cell closed apically before recurrent vein. Parallel vein (CU1a) interstitial. Hind wing 5.5-6.0 times longer than its maximum width. First costal abscissa $(\mathrm{C}+\mathrm{SC}+\mathrm{R})$ $0.7-1.0$ times as long as second abscissa (1-SC+R). First abscissa of mediocubital vein (M+CU) 0.45 0.50 times as long as second abscissa $(1 \mathrm{M})$. Recurrent vein $(\mathrm{m}-\mathrm{cu})$ very short, unsclerotised and not pigmented, distinctly antefurcal, strongly oblique towards base of wing.

Legs. Fore tibia anterior margin with short, rather thick, sparse spines arranged in single line. Segments of middle tarsus considerably longer than their width. Hind coxa short, shortly oval to subround, without basoventral corner and tubercle, 1.3 times longer than maximum width. Hind femur narrow, elongateoval, 3.8-4.5 times longer than wide. Hind tibia with several slender spines on outer distal margin. Hind tarsus 1.1 times longer than hind tibia. Hind basitarsus $0.55-0.60$ times as long as remaining segments combined. Second segment of hind tarsus 0.6 times as long as basitarsus, 1.5-1.7 times longer than narrow fifth segment (without pretarsus). Tarsal segments slender. Claws short, thick basally, with short and weakly curved distal part.

Metasoma. Petiole in lateral view, ventrally almost straight, dorsally very weakly arched in anterior half and almost straight in posterior half, highest in anterior fourth; in dorsal view, petiole rather slender, subparallel-sided or very weakly and evenly widening towards apex, without spiracular tubercles and dorsope. Length of petiole 2.8-3.4 times its posterior width, 1.5-1.6 times length of propodeum; posterior width 1.9-2.2 times minimum anterior width. Second to sixth tergites with laterotergites separated; second and third separated laterotergites not fused. Second suture very shallow, narrow, evenly curved. Median length of second tergite 1.3-1.4 times its anterior width, 1.6-1.7 times length of third tergite. Median length of second and third tergites combined 2.1-2.4 times its anterior width, 0.8-0.9 times their maximum width. Ovipositor straight. Ovipositor sheath 1.5-2.2 times longer than petiole, $0.4-0.7$ times as long as metasoma, $0.8-1.25$ times as long as mesosoma, $0.4-0.7$ times as long as fore wing.

SCULPTURE AND PUBESCENCE. Head entirely densely small reticulate-granulate. Mesosoma almost entirely densely reticulate-granulate, propodeum without areas, with long, distinct, complete longitudinal lateral carinae weakly convergent backwards. Hind coxa entirely coriaceous; hind femur dorsally and laterally coriaceous, smooth ventrally. Petiole granulate-reticulate, second and third tergites entirely densely small reticulate-areolate. Fourth to sixth tergites entirely of almost entirely densely and finely coriaceous. Vertex mainly glabrous, with short white setae laterally. Mesoscutum mainly glabrous, with very short and sparse white setae arranged along notauli and laterally. Setae of hind tibia semi-erect, short and rather sparse, their length on dorsal surface 0.3-0.4 times maximum width of tibia.

CoLour. Body mainly reddish brown to dark reddish brown, rarely mainly pale reddish brown or reddish brown, with pale spots; head always in lower half or entirely paler, mainly yellowish brown; mesosoma almost darker to black below. Palpi brown to dark brown, but sometimes paler basally. Antenna yellow to brownish yellow in basal two-third, 5-12 ${ }^{\text {th }}$ apical segments dark brown to black. Legs mainly brownish yellow or light reddish brown to dark reddish brown or almost black; hind tibia sometimes reddish 
brown, always pale (yellow or pale reddish brown) basally on short distance; all tarsi mainly yellow with faint infuscation, apical segments entirely and often anterior half of hind basitarsus dark to dark brown. Ovipositor sheath brownish yellow or pale reddish brown in anterior $0.25-0.50$, brown to black in posterior half. Fore wing strongly darkened, with contrasting small hyaline spots and stripes (Fig. 15A). Pterostigma dark brown in apical 0.5 , whitish yellow or white in basal half.

\section{Male \\ Unknown.}

\section{Distribution}

The UAE, Yemen.

Genus Spathius Nees, 1819

\section{Type species}

Cryptus clavatus Panzer, 1809 (= Ichneumon exarator Linnaeus, 1758), by monotypy.

\section{Remarks}

Spathius is one of the largest genera of the subfamily Doryctinae, including over 450 described and a vast number of undescribed species (Zaldívar-Riverón et al. 2018). This genus is widely distributed in the five continents, although most of its species richness is concentrated in the Afrotropical and Oriental regions (with more than 350 described species).

The genus Spathius has not previously been recorded for the Arabian Peninsula, in spite of its intermediate position between the Africa and Oriental Asia - with both being the most species rich continents for the genus.

\section{Distribution}

All zoogeographical regions.

\section{Key to Arabian species of the genus Spathius}

1. Face with very dense and rather fine aciculate sculpture (like the surface of a CD disk) (Figs 16B, 24B)

- Face never with the above type of sculpture, usually rugose-striate or reticulate (Figs 18G, 20B, 22B)

2. Vertex finely transversely striate in median anterior half, smooth laterally and posteriorly (Fig. 24D). Eye glabrous. POL almost equal to Od (Fig. 24D). Antenna long, almost as long as body (Fig. 24A). Praescutellar depression of mesosoma with six-seven complete carinae (Fig. 24H). Nervulus (cu-a) of fore wing distinctly postfurcal (Fig. 25A). Brachial (subdiscal) cell closed apically weakly behind recurrent vein; parallel vein (CU1a) arising almost from anterior third of the vein (3-CU1) closed brachial (subdiscal) cell distally (Fig. 25A). Medio-basal carina of propodeum long, about 1.5 times longer than anterior fork of areola (Fig. 24J). Length of setae on dorsal surface of hind tibia 0.9-1.3 times maximum width of tibia (Fig. 24G). Petiole in lateral view dorsally arched in anterior third (Fig. 25C). Body length 3.6-4.4 mm. - UAE S. subafricanus Belokobylskij sp. nov.

- Vertex entirely smooth (Fig. 16C). Eye with short and sparse pale setae. POL 1.7 times Od (Fig. 16C). Antenna short, 0.7 times as long as body(Fig. 16A). Praescutellar depression of mesosoma with only three complete carinae (Fig. 16G). Nervulus (cu-a) of fore wing interstitial or very weakly antefurcal (Fig. 17A). Brachial (subdiscal) cell closed apically distinctly behind recurrent vein; parallel vein 
(CU1a) arising almost from middle of the vein (3-CU1) closed brachial (subdiscal) cell distally (Fig. 17A). Petiole in lateral view dorsally arched submedially (Figs 16L, 17D). Medio-basal carina of propodeum short, 0.5 times as long as anterior fork of areola (Fig. 16G). Length of setae on dorsal surface of hind tibia 0.4-0.8 times maximum width of tibia (Fig. 16J). Body length $4.5 \mathrm{~mm}$. - Yemen S. alkadanus Belokobylskij sp. nov.

3. Metasoma behind petiole with dense small reticulate-areolate sculpture (Fig. 21C). Second to fourth metasomal tergites with separated laterotergites (Fig. 21D). Maxillary palp entirely dark reddish brown (Fig. 20C). Vertex entirely very densely granulate reticulate (Fig. 20D). Body length 3.6$4.3 \mathrm{~mm}$. - Yemen

S. lahji Belokobylskij sp. nov.

- Metasoma behind petiole smooth (Figs 19B, 23C). Only second metasomal tergite with separated laterotergite (Figs 19C, 23D). Maxillary palp yellow or yellowish brown (Figs 18C, 22D). Vertex mainly or entirely smooth (Figs 18B, 22C)

4. Hypoclypeal depression large and distinctly transverse, its width about twice distance from depression to eye (Fig. 22B). First (r) and second (3-SR) radial abscissae of fore wing forming almost single line. Parallel vein (CU1a) not interstitial, arising from anterior third of the vein (3-CU1) closed brachial (subdiscal) cell distally (Fig. 23A). Petiole long, its length 2.6-2.9 times posterior width, 1.8-2.0 times length of propodeum (Fig. 23B-C). Ovipositor long, its sheath 1.3-1.6 times longer than metasoma, 1.0-1.1 times as long as fore wing (Fig. 22A). Body length 3.0-3.4 mm. - Japan, UAE, Yemen

S. nixoni Belokobylskij \& Maetô, 2009

- Hypoclypeal depression medium-sized and rounded, its width 0.8 times distance from depression to eye (Fig. 18G). First (r) and second (3-SR) radial abscissae of fore wing forming distinctly obtuse angle. Parallel vein (CU1a) interstitial (Fig. 19A). Petiole short, its length 1.85 times posterior width, 1.6 times length of propodeum (Fig. 19B-C). Ovipositor short, its sheath 0.33 times as long as metasoma, 0.2 times as long as fore wing (Fig. 18A). Body length $2.6 \mathrm{~mm}$. - Yemen

S. austroarabicus Belokobylskij sp. nov.

Spathius alkadanus Belokobylskij sp. nov.

urn:1sid:zoobank.org:act:7388A7AB-A064-4760-8CB1-EF22363E2B25

Figs 16-17

\section{Comparative diagnosis}

This new species belongs to the $S$. vulnificus species-group and is very similar to $S$. subafricanus sp. nov. The differences between these species are shown in the below key.

\section{Etymology}

This new species is named after its type locality, Al Kadan.

\section{Material examined}

Holotype

YEMEN • O; "Yemen (6090), Al Kadan, x.2001, light trap, A.v. Harten, T. Abdul-Haq. RMNH'02"; RMNH.

\section{Description}

\section{Female}

Measurements. Body length $4.5 \mathrm{~mm}$; fore wing length $2.4 \mathrm{~mm}$.

HEAD. Head width (dorsal view) 1.4 times its median length, 1.2 times width of mesoscutum. Vertex weakly convex. Head behind eyes (dorsal view) subparallel in anterior half and evenly roundness 


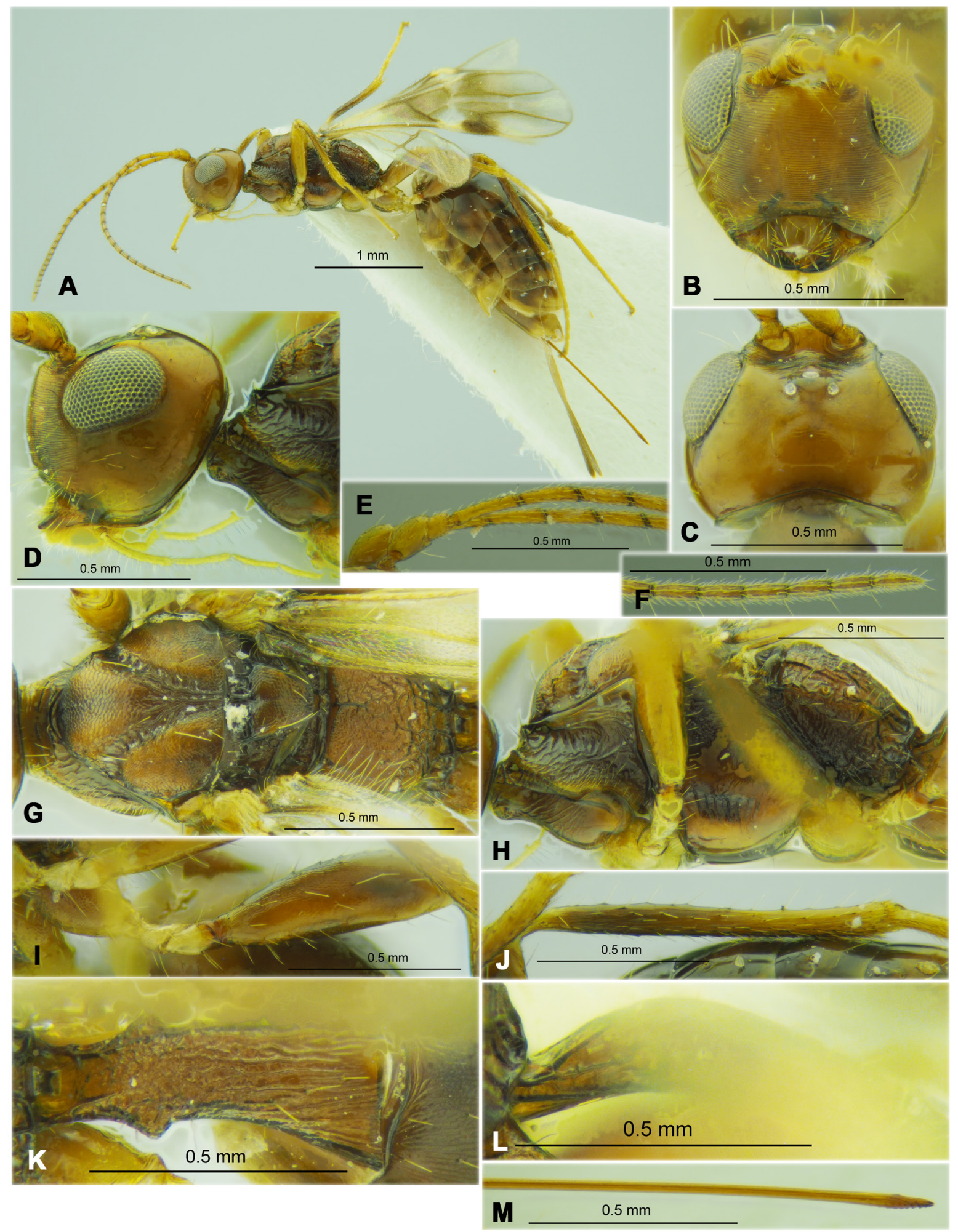

Fig. 16. Spathius alkadanus Belokobylskij sp. nov., holotype, + (RMNH). A. Habitus, lateral view. B. Head, front view. C. Head, dorsal view. D. Head, lateral view. E. Basal segments of antenna. F. Apical segments of antenna. G. Mesosoma, dorsal view. H. Mesosoma, lateral view. I. Hind coxa and femur. J. Hind tibia. K. Petiole of metasoma, dorsal view. L. Petiole of metasoma, lateral view. M. Ovipositor. 

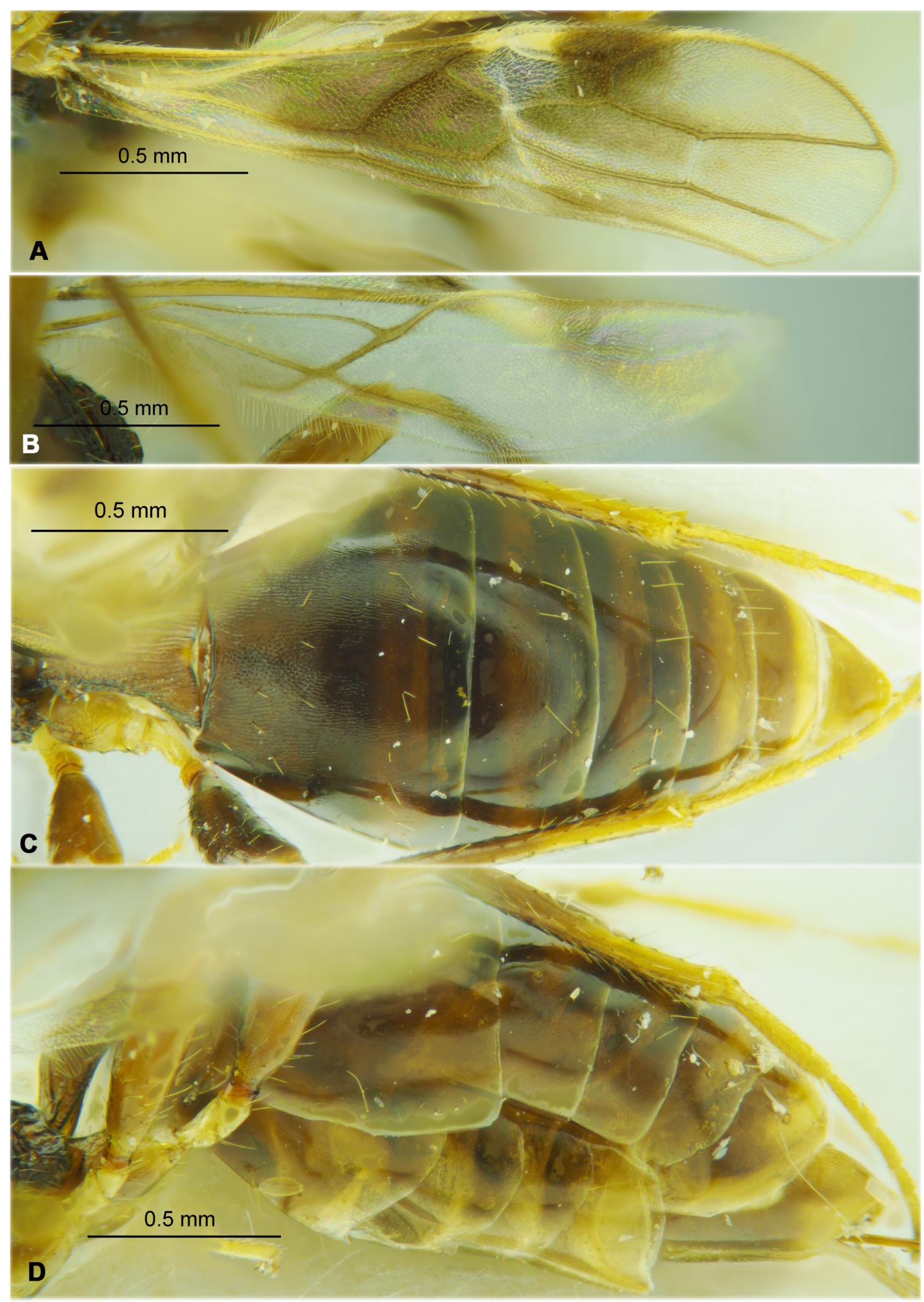

Fig. 17. Spathius alkadanus Belokobylskij sp. nov., holotype, $q$ (RMNH). A. Fore wing. B. Hind wing. C. Metasoma, dorsal view. D. Metasoma, lateral view. 
decreased in posterior half. Transverse diameter of eye (dorsal view) 1.4 times longer than temple. Ocelli small, in triangle with base 1.3 times its sides. POL 1.7 times Od, 0.55 times OOL. Eye with short and sparse pale setae, in lateral view distinctly oblique, its maximum diameter 1.3 times minimum diameter. Malar space 0.65 times maximum diameter of eye, 1.2 times basal width of mandible. Face weakly convex, its width 1.1 times maximum diameter of eye, almost equal to height of face and clypeus combined. Clypeal suture upper absent on wide distance, distinct laterally at rather long lower part. Ventral margin of clypeus with distinct narrow flange. Hypoclypeal depression medium-sized and round, its width 0.6 times shortest distance from edge to eye, 0.4 times width of face. Occipital carina dorsally complete and almost not broken toward ocellar triangle, not reaching below hypostomal carina and obliterated relatively long distance before mandible base. Hypostomal flange narrow.

AnTEnNA. Antenna slender, filiform, 24-segmented, 0.7 times as long as body. Scape 1.8 times longer than its maximum width. First flagellar segment 4.8 times longer than apical width, 1.15 times as long as second segment. Penultimate segment 3.0 times longer than width, 0.5 times as long as first segment, 0.9 times as long as apical segment; latter weakly acuminate apically and without spine.

Mesosoma. Mesosoma not depressed, its maximum length 1.8 times maximum height. Pronotal carina distinct, situated very closely to posterior margin of pronotum on wide distance. Pronotal lateral depression distinct, wide, shallow, finely crenulate, delineated upper and below by carinae. Mesoscutum (lateral view) high, subroundly and curvedly elevated above pronotum, its median lobe (dorsal view) distinctly convex anteriorly and without anterolateral corners, without median longitudinal furrow; mesoscutum in dorsal view 0.9 times as long as wide. Notauli complete, wide, deep anteriorly and shallow posteriorly, densely crenulate. Prescutellar depression rather shallow, wide, with three complete carinae, 0.3 times as long as scutellum. Scutellum weakly convex, with distinct lateral carinae, almost as long as maximum anterior width. Subalar depression wide, shallow, distinctly rugulose-striate. Precoxal sulcus deep, wide, short, densely crenulate, running along anterior 0.5 of lower part of mesopleuron. Postpectal carina absent. Metanotum with very low, wide and apically rounded tubercle (lateral view). Metapleural flange (lobe) rather wide, distinct, subrounded distally. Propodeum evenly and weakly roundly convex, almost without lateral tubercles.

WINGS. Fore wing 3.4 times longer than wide. Pterostigma rather wide, 3.7 times longer than its maximum width. Radial vein ( $\mathrm{r}$ ) arising distinctly behind middle of pterostigma, from basal 0.6. Radial (marginal) cell not shortened, metacarpus (1-R1) 1.3 times longer than pterostigma. Second radial abscissa (3-SR) 3.0 times longer than first abscissa (r) and forming with it weakly obtuse angle, 0.55 times as long as the weakly curved third abscissa (SR1), 0.9 times longer than straight first radiomedial vein (2-SR). Second radiomedial (submarginal) cell very weakly narrowed distally, its length 2.8 times maximum width, 1.4 times length of brachial (subdiscal) cell. Second abscissa of medial vein (2-SR $+\mathrm{M})$ rather long, 0.8 times as long as recurrent vein (m-cu). Nervulus (cu-a) interstitial or very weakly antefurcal. Mediocubital vein $(\mathrm{M}+\mathrm{CU} 1)$ very weakly sinuate to longitudinal anal vein (1-1A). Narrow brachial (subdiscal) cell closed apically distinctly behind recurrent vein (m-cu). Parallel vein (CU1a) not interstitial, arising almost from middle of the vein (3-CU1) closed brachial (subdiscal) cell distally. Hind wing 4.7 times longer than its maximum width. First costal abscissa $(\mathrm{C}+\mathrm{SC}+\mathrm{R}) 0.6$ times as long as second abscissa $(1-\mathrm{SC}+\mathrm{R})$. First abscissa of mediocubital vein $(\mathrm{M}+\mathrm{CU}) 0.6$ times as long as second and third abscissa (1-M) combined. Recurrent vein (m-cu) rather short, sclerotised but unpigmented, subinterstitial, curved towards base of wing.

LEGS. Fore tibia anterior margin with short and dense spines arranged in single vertical line. Segments of middle tarsus distinctly longer than their width. Hind coxa rather short, with basoventral corner and small tubercle, 1.3 times longer than its maximum width. Hind femur thickened, elongate-oval, 3.5 times longer than wide. Hind tibia with four slender spines on outer distal margin. Hind tarsus 0.9 
times as long as hind tibia. Hind basitarsus 0.7 times as long as remaining segments combined. Second segment of hind tarsus 0.5 times as long as basitarsus, 1.4 times longer than fifth segment (without pretarsus). Tarsal segments rather slender.

Metasoma. Petiole in lateral view, ventrally distinctly evenly curved, dorsally distinctly arched submedially and almost straight on remaining part, highest in anterior third; in dorsal view, petiole distinctly evenly widening from base towards its apex, with distinct spiracular tubercles in anterior third and fine dorsope. Length of petiole 1.65 times its posterior width, 1.6 times length of propodeum; posterior width 1.7 times its width at spiracular level, 2.7 times its minimum anterior width. Second tergite with separated laterotergites; following tergites without separated laterotergites; laterotergites of second and third tergites fused, without suture. Second suture almost absent. Median length of second and third tergites combined 1.3 times anterior width of second tergite, 0.7 times their maximum width. Ovipositor straight. Ovipositor sheath about 2.0 times longer than petiole, 0.5 times as long as metasoma, as long as mesosoma, 0.55 times as long as fore wing.

SCUlPTURE AND PUBESCENCE. Vertex entirely smooth; frons distinctly and obliquely striate, smooth on narrow median vertical stripe; face entirely with very dense and thin aciculation; temple entirely smooth. Mesoscutum densely and finely granulate-reticulate, with two distinctly convergent posteriorly median carinae in posterior half and rugulosity between carinae; scutellum finely reticulate, more distinct in posterior quarter. Mesopleuron widely smooth, with fine reticulation with striation medially vertically, upper and lower parts. Propodeum entirely rugose-areolate, with fine granulation anteriorly, with areas rather distinctly delineated by carinae, medio-basal carina in anterior third of propodeum short, 0.5 times as long as anterior fork of areola; areola long and narrow, 2.8 times longer than its maximum width. Hind coxa densely rugulose, with additional striation dorsally, rugulose-reticulate laterally; hind femur dorsally reticulate, finely to very finely reticulate-coriaceous laterally, smooth below. Petiole distinctly and densely striate, with dense rugosity in anterior half. Second and third in anterior half tergites densely and rather fine reticulate- aciculate, third tergite smooth in posterior half. Remaining tergites entirely smooth. Vertex medially glabrous, with rather long and very sparse yellow setae laterally and posteriorly. Mesoscutum with long and sparse white semi-erect setae arranged along notauli and laterally, glabrous on wide median areas of all lobes. Setae of hind tibia semi-erect, medium length and rather sparse, their length on dorsal surface $0.4-0.8$ times maximum width of tibia.

CoLour. Head light reddish brown and infuscate dorsally, mesosoma and metasoma mainly reddish brown, with yellowish spots or stripes. Palpi yellow. Antenna brownish yellow in basal two thirds, infuscate in apical one-thirds. Legs mainly light reddish brown trochanters, trochantelli and tarsi yellow to brownish yellow, all tibiae yellow on short basal part. Ovipositor sheath pale yellow in anterior half and brown in posterior half. Fore wing finely maculate especially along veins, with several hyaline spots. Pterostigma mainly dark brown, pale yellow in basal half.

\section{Male}

Unknown.

\section{Distribution}

Yemen.

\section{Remarks}

This is the first record of this species-group for the Afrotropical region, having the face with very dense, fine and absolutely even transverse aciculation (like the surface of a CD). 
Spathius austroarabicus Belokobylskij sp. nov. urn:1sid:zoobank.org:act:7D9A7222-329E-46BD-A938-1E5609144351

Figs $18-19$

\section{Comparative diagnosis}

This species belongs to the $S$. apidanus species-group (formerly a monotypic group from the Aru Islands) according to the position of its recurrent vein (m-cu) of the fore wing, arising from the parallel vein (CU1a) at a long distance from the apex of the brachial (subdiscal) cell, and the entirely smooth metasomal tergites behind the petiole. This new species distinctly differs from S. apidanus Nixon, 1943 by several diagnostic characters: vertex smooth (striate in S. apidanus), pronotal keel widely fused with posterior margin of pronotum in dorsal view (free and widely separated in S. apidanus), mesoscutum without transverse rugae on lateral lobes (with distinct additional rugae in $S$. apidanus), hind femur elongate-oval (claviform in $S$. apidanus), fore wing hyaline (dark brown with submedian pale facia in $S$. apidanus), petiole of metasoma rather short and wide, about 1.5 times longer than propodeum (very long and narrow, about 2.5 times longer in $S$. apidanus), ovipositor sheath distinctly shorter than metasoma (as long as body in S. apidanus), and body brownish yellow (dark brown in S. apidanus).

Spathius austroarabicus sp. nov. may also include in the $S$. capaneus species-group in the section with a smooth tergite behind the petiole, having the hind tibia clothed only with very short setae, which are much shorter than the width of the tibia. This new species is similar in this group to $S$. semele Nixon, 1943, from Philippines, but differs from it by having the recurrent vein (m-cu) of the fore wing arising from the apex of brachial (subdiscal) cell (recurrent vein (m-cu) arising from the parallel vein (CU1a) at a long distance from the apex of the brachial (subdiscal) cell in S. semele), hind coxa and tibia entirely yellow (coxa brown and tibia darkened in posterior 0.7 in $S$. semele), and ovipositor sheath distinctly shorter than the metasoma (as long as body in S. semele).

\section{Etymology}

Named from the combination of the Latin words 'australis' (= south) and 'arabicus' (= Arabian), since this species was found in the southern part of the Arabian Peninsula.

\section{Material examined}

\section{Holotype}

YEMEN - O+; "Yemen (no 2989), Ta'izz, light trap, 1-3.iv.1998, A.v. Harten \& Ahmad Ahwad, RMNH'98"; RMNH.

\section{Description}

Female

MeAsurements. Body length $2.6 \mathrm{~mm}$; fore wing length $2.0 \mathrm{~mm}$.

HeAd. Head width (dorsal view) 1.4 times its median length, 1.2 times width of mesoscutum. Vertex weakly convex. Head behind eyes (dorsal view) rather weakly and evenly roundness decreased. Transverse diameter of eye (dorsal view) 1.8 times longer than temple. Ocelli small, in almost equilateral triangle. POL almost equal to Od, 0.25 times OOL. Eye glabrous, in lateral view oblique, its maximum diameter 1.3 times minimum diameter. Malar space 0.8 times maximum diameter of eye, almost equal to basal width of mandible. Face weakly convex, its width 1.2 times maximum diameter of eye, 1.2 times height of face and clypeus combined. Clypeal suture almost complete, distinct laterally but very fine dorsally. Ventral margin of clypeus with distinct narrow flange. Hypoclypeal depression medium-sized and rounded, its width 0.8 times shortest distance from edge to eye, 0.4 times width of face. Occipital carina dorsally complete and not broken toward ocellar triangle, not reaching below hypostomal carina and obliterated rather long distance before mandible base. Hypostomal flange narrow. 
AnTENNA. Atenna slender, weakly setiform, 21-segmented, 1.1 times longer than body. Scape 1.3 times longer than its maximum width. First flagellar segment 6.0 times longer than apical width, 0.95 times as long as second segment. Penultimate segment 4.7 times longer than width, 0.7 times as long as first segment, 0.9 times as long as apical segment; latter acuminate apically and without spine.

Mesosoma. Mesosoma not depressed, its maximum length 1.8 times its maximum height. Pronotal carina distinct, its anterior branch situated submedially and far separated from posterior margin of pronotum. Pronotal lateral depression distinct, densely crenulate, delineated below by carinae. Mesoscutum (lateral

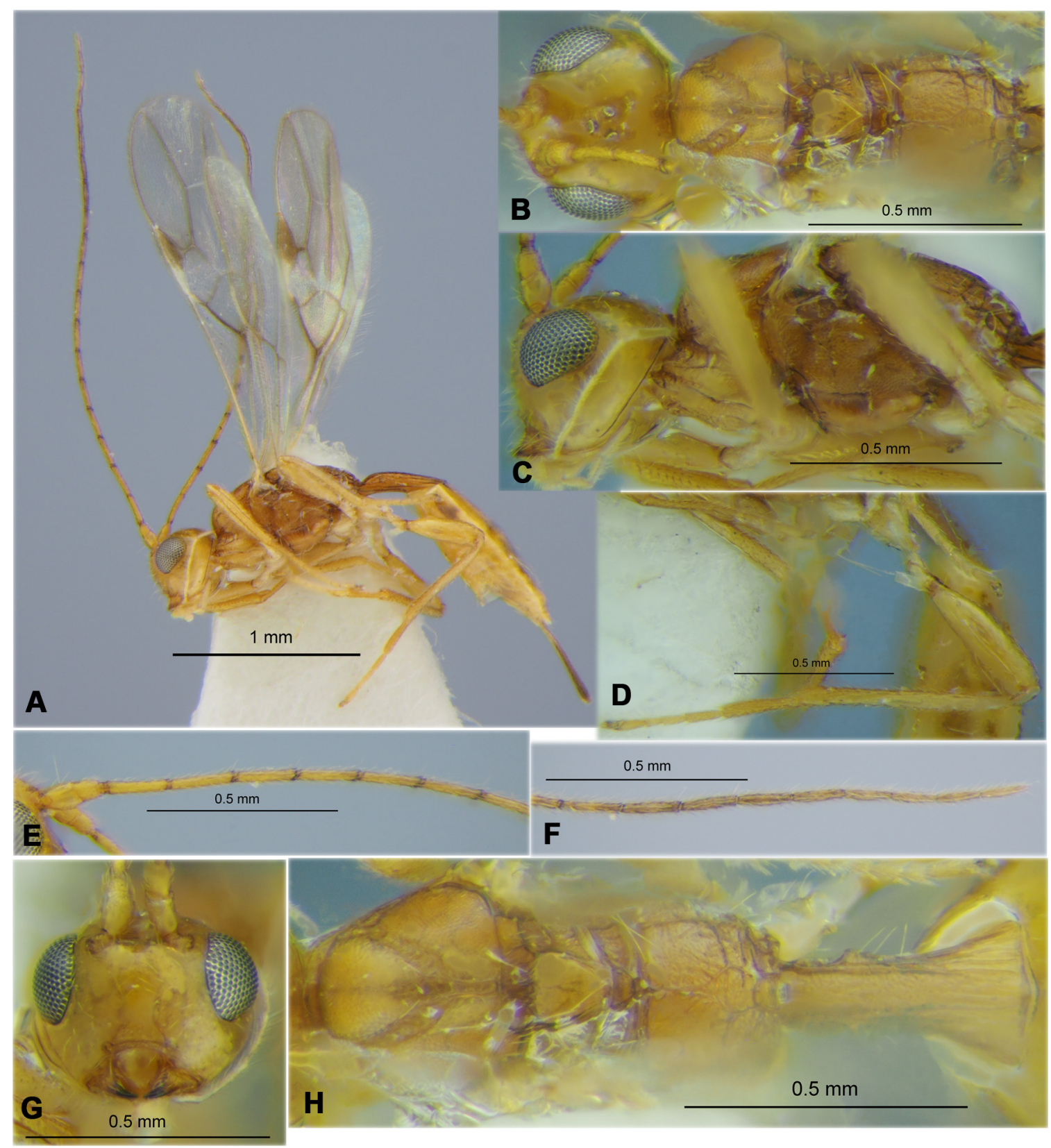

Fig. 18. Spathius austroarabicus Belokobylskij sp. nov., holotype, $q$ (RMNH). A. Habitus, lateral view. B. Head and mesosoma, dorsal view. C. Head and mesosoma, lateral view. D. Hind leg. E. Basal segments of antenna. F. Apical segments of antenna. G. Head, front view. H. Mesosoma and petiole, dorsal view. 
view) high, subroundly and curvedly elevated above pronotum, its median lobe (dorsal view) distinctly convex anteriorly and without anterolateral corners, with rather distinct and sculptured longitudinal median furrow in posterior half; mesoscutum in dorsal view 0.9 times as long as wide. Notauli complete, rather wide, deep anteriorly and shallow posteriorly, sparsely crenulate. Prescutellar depression rather deep, relatively wide, with distinct median carina, finely rugulose-carinate, 0.4 times as long as scutellum. Scutellum weakly convex, with distinct lateral carinae, almost as long as its maximum anterior width. Subalar depression rather wide, shallow, reticulate-rugulose. Precoxal sulcus deep, wide, short, densely crenulate, running along anterior 0.4 of lower part of mesopleuron. Postpectal carina absent. Metanotum with low, wide below and distally acuminate medio-dorsal tubercle (lateral view). Metapleural flange (lobe) narrow, distinct, subrounded. Propodeum roundly convex, without lateral tubercles.
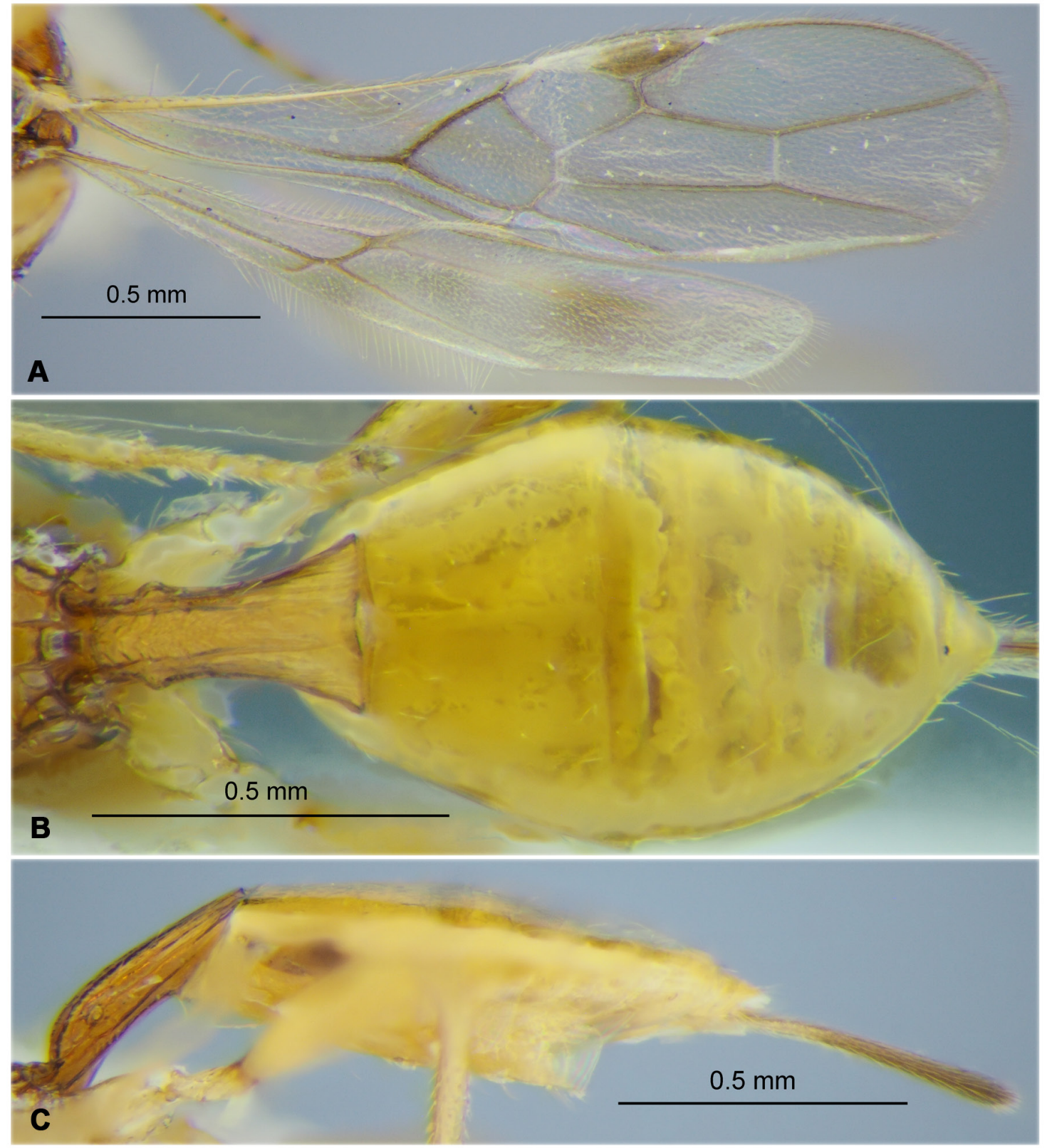

Fig. 19. Spathius austroarabicus Belokobylskij sp. nov., holotype, $q$ (RMNH). A. Wings. B. Metasoma, dorsal view. C. Metasoma and ovipositor, lateral view. 
Wings. Fore wing 3.8 times longer than wide. Pterostigma wide, 3.6 times longer than its maximum width. Radial vein (r) arising almost from middle of pterostigma. Radial (marginal) cell not shortened, metacarpus (1-R1) 1.4 times longer than pterostigma. Second radial abscissa (3-SR) 5.5 times longer than first abscissa (r) and forming with it weakly obtuse angle, 0.6 times as long as the mainly straight, but weakly curved apically third abscissa (SR1), 1.3 times as long as straight first radiomedial vein (2SR). Second radiomedial (submarginal) cell weakly narrowed distally, its length 3.0 times maximum width, 1.9 times length of brachial (subdiscal) cell. Second abscissa of medial vein (2-SR+M) long, 0.7 times as long as recurrent vein (m-cu). Nervulus (cu-a) almost interstitial. Mediocubital vein (M+CU1) weakly sinuate and almost not curved to longitudinal anal vein (1-1A). Narrow brachial (subdiscal) cell closed apically weakly before recurrent vein(m-cu). Parallel vein (CU1a) interstitial. Hind wing 6.2 times longer than its maximum width. First costal abscissa $(\mathrm{C}+\mathrm{SC}+\mathrm{R}) 0.4$ times as long as second abscissa (1-SC+R). First abscissa of mediocubital vein $(\mathrm{M}+\mathrm{CU}) 0.5$ times as long as second and third abscissa (1-M) combined. Recurrent vein (m-cu) short, unsclerotised but pigmented, weakly antefurcal, curved towards base of wing.

Legs. Fore tibia anterior margin with short, rather dense spines arranged in long single line. Segments of middle tarsus distinctly longer than their width. Hind coxa rather short, with basoventral corner and tubercle, 1.4 times longer than maximum width. Hind femur thickened, elongate-oval, 3.4 times longer than wide. Hind tibia with several slender spines on outer distal margin. Hind tarsus 0.85 times as long as hind tibia. Hind basitarsus thickened, 0.6 times as long as remaining segments combined. Second segment of hind tarsus 0.5 times as long as basitarsus, 1.1 times longer than fifth segment (without pretarsus). Tarsal segments mainly slender.

Metasoma. Petiole in lateral view, ventrally almost straight, dorsally weakly arched in anterior half and almost straight in posterior half, highest in anterior fourth; in dorsal view, petiole rather slender, distinctly evenly widening towards apex in posterior third, with distinct spiracular tubercles in anterior third and fine dorsope. Length of petiole 1.85 times its posterior width, 1.6 times length of propodeum; its posterior width 1.6 times its width at spiracular level, 2.5 times its minimum anterior width. Second to sixth tergites without laterotergites separated; second and third lateral parts of tergites fused. Second suture absent. Median length of second and third tergites combined 1.1 times anterior width of second tergite, 0.6 times their maximum width. Ovipositor straight. Ovipositor sheath as long as petiole, 0.33 times as long as metasoma, 0.55 times as long as mesosoma, 0.2 times as long as fore wing.

SCulpture AND PUBESCEnCE. Head mainly smooth, with fine and short aciculation near ocelli; face smooth medially, finely and obliquely aciculate laterally and transversely ventrally. Mesoscutum densely granulate, with two convergent posteriorly distinct longitudinal carinae in posterior half and rugulosity between carinae; scutellum entirely and distinctly granulate. Mesopleuron mainly and densely granulatereticulate, almost smooth below precoxal sulcus. Propodeum with areas distinctly delineated by carinae, almost entirely transversely striate-rugulose; medio-basal carina medium length, 1.5 times as long as anterior fork of areola; areola short and wide, 1.3 times as long as maximum width. Hind coxa weakly rugulose- coriaceous in anterior half and smooth on other part; hind femur mainly smooth, dorsally partly rugulose-aciculate. Petiole distinctly striate with long and subparallel dorsal carinae, densely rugulose-reticulate in medial area in anterior half. Remainder tergites entirely smooth. Vertex mainly glabrous, with short and sparse white setae laterally. Mesoscutum mainly glabrous, with long and sparse white setae arranged along notauli and laterally. Setae of hind tibia semi-erect, short and rather dense, their length on dorsal surface about 0.7 times maximum width of tibia.

CoLour. Head dorsally, mesosoma and petiole yellowish brown; head in lowed half and metasoma behind petiole yellow. Palpi pale yellow. Antenna yellow basally, mainly pale brown to dark brown apically. 
Legs mainly brownish yellow, all coxae yellow. Ovipositor sheath dark brown, but pale anteriorly. Fore wing hyaline. Pterostigma dark brown medially, whitish or pale yellow in basal 0.4 and shortly apically.

\section{Male \\ Unknown.}

\section{Distribution}

Yemen.

Spathius lahji Belokobylskij sp. nov. urn:1sid:zoobank.org:act:3E094E2E-9F85-48D1-9D8B-B58ED8226E0C

Figs $20-21$

\section{Comparative diagnosis}

According to Nixon's (1943) key, this new species belongs to the $S$. dissors Wilkinson species-group being similar to $S$. zagreus Nixon, 1943 from Philippines. However, S. lahji sp. nov. distinctly differs from $S$. zagreus by having all maxillary palpal segments entirely dark reddish brown (apical segment whitish in $S$. zagreus), fore and middle tibiae entirely black (pale at apex in S. zagreus), vertex entirely and very densely granulate reticulate (with distinct traces of transverse aciculation in S. zagreus), temple very densely punctate-reticulate (virtually smooth in $S$. zagreus), mesonotum with low but distinct longitudinal keel (without keel and flat in $S$. zagreus), mesopleuron entirely finely reticulate-coriaceous (with broad smooth shining area in S. zagreus), second-fourth metasomal tergites with fine but distinct lateral margins (without such margins in S. zagreus), and ovipositor sheaths very short, about 0.3 times as long as metasoma (long and 0.8-0.9 times as long as metasoma in S. zagreus).

\section{Etymology}

Named after the type locality of the new species, Lahj in Yemen.

\section{Material examined}

\section{Holotype}

YEMEN • +; “Yemen (6022), Lahj, vii-ix.2001, Mal. trap, A.v. Harten \& A. Sallam, RMNH'02”; RMNH.

\section{Paratype}

YEMEN • $1 \delta^{\uparrow}$; same label data as for holotype; RMNH.

\section{Description}

\section{Female}

Measurements. Body length $3.6 \mathrm{~mm}$; fore wing length $2.2 \mathrm{~mm}$.

HeAD. Head width (dorsal view) 1.6 times its median length, 1.2 times width of mesoscutum. Vertex convex. Head behind eyes (dorsal view) weakly convex anteriorly and weakly evenly roundness decreased posteriorly. Transverse diameter of eye (dorsal view) 1.4 times longer than temple. Ocelli small, in almost equilateral triangle. POL 1.3 times Od, 0.5 times OOL. Eye with very short and sparse setae, in lateral view oblique, its maximum diameter 1.2 times minimum diameter. Malar space 0.4 times maximum diameter of eye, 0.85 times basal width of mandible. Face weakly convex, its width 0.9 times maximum diameter of eye, 1.4 times height of face and clypeus combined. Clypeal suture complete, distinct laterally but fine mediodorsally. Ventral margin of clypeus with narrow flange. Hypoclypeal depression medium-sized and rounded, its width 0.8 times shortest distance from edge to eye, 0.4 times 


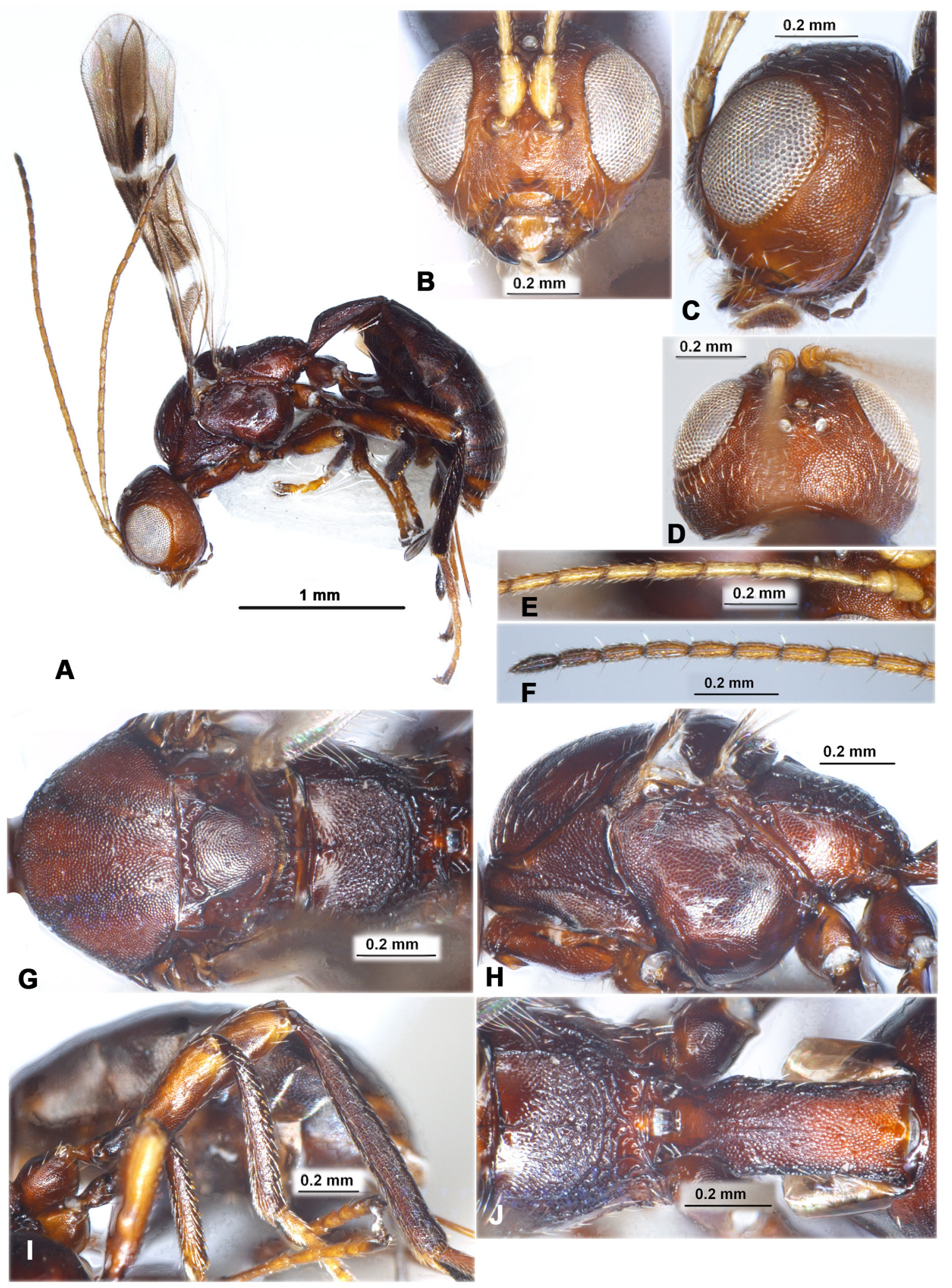

Fig. 20. Spathius lahji Belokobylskij sp. nov., holotype, $q$ (RMNH). A. Habitus, lateral view. B. Head, front view. C. Head, lateral view. D. Head, dorsal view. E. Basal segments of antenna. F. Apical segments of antenna. G. Mesosoma, dorsal view. H. Mesosoma, lateral view. I. Hind leg. J. Propodeum and petiole of metasoma, dorsal view. 

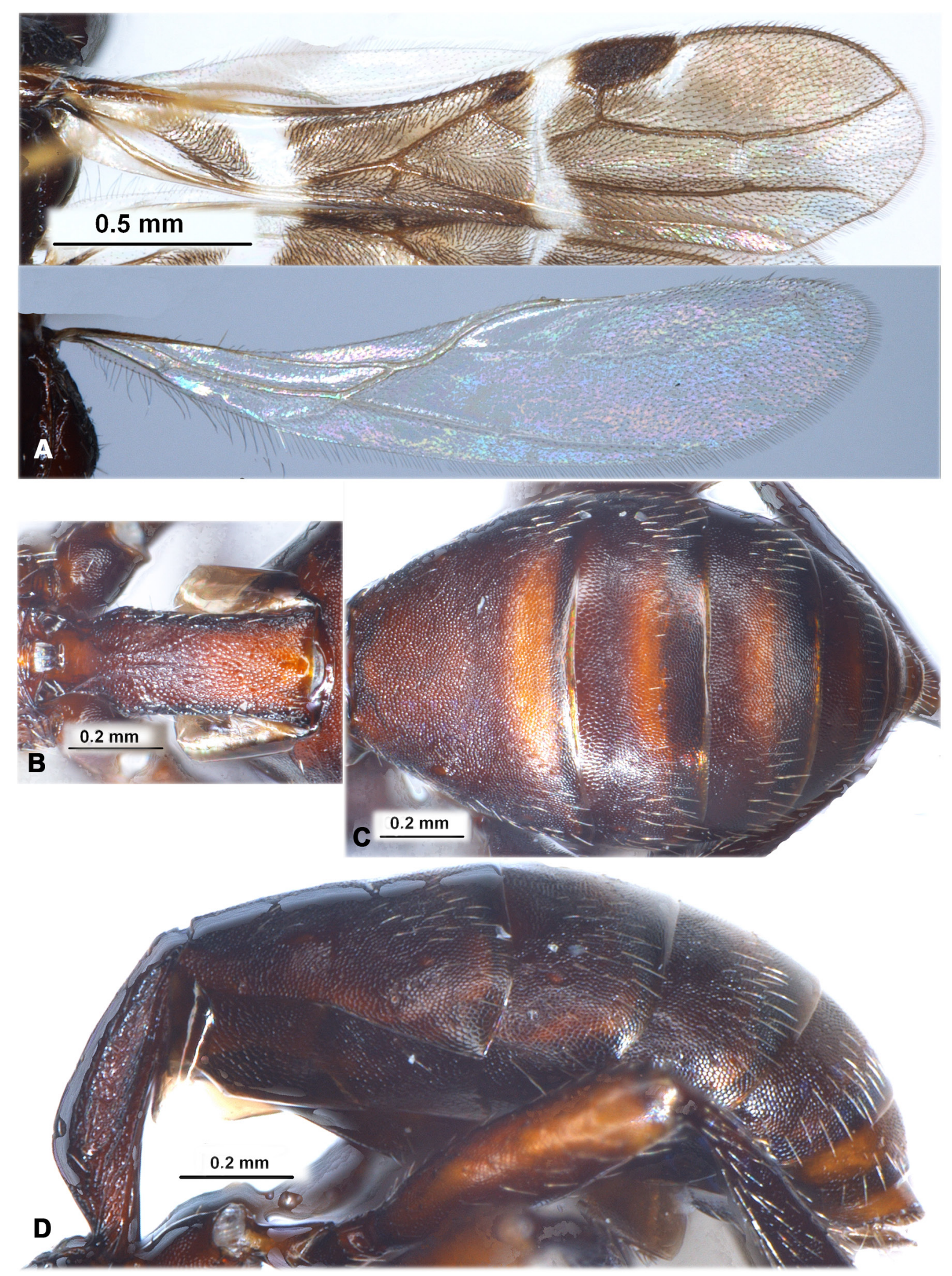

Fig. 21. Spathius lahji Belokobylskij sp. nov., holotype, $q(\mathrm{RMNH})$. A. Wings. B. Petiole of metasoma, dorsal view. C. Metasoma without petiole, dorsal view. D. Metasoma, lateral view. 
width of face. Occipital carina dorsally complete and almost not broken toward ocellar triangle, not reaching below hypostomal carina and obliterated short distance before mandible base. Hypostomal flange narrow.

Antenna. Antenna rather slender, filiform, 22-segmented, 0.7 times as long as body. Scape 1.6 times longer than its maximum width. First flagellar segment 3.5 times longer than apical width, about as long as second segment. Penultimate segment 3.0 times longer than width, 0.7 times as long as first segment, 0.9 times as long as apical segment; latter acuminate apically and without spine.

Mesosoma. Mesosoma high and short, its maximum length 1.6 times its maximum height. Pronotal carina fine to very fine, its posterior fused medially with posterior margin of pronotum. Pronotal lateral depression shallow and narrow, reticulate-crenulate, delineated below by carina. Mesoscutum (lateral view) high, roundly and curvedly elevated above pronotum, its median lobe (dorsal view) distinctly convex anteriorly and without anterolateral corners, without longitudinal median furrow; mesoscutum in dorsal view 0.9 times as long as maximum width. Notauli incomplete, rather narrow, rather distinct and finely crenulate in anterior half, very shallow or almost indistinct in posterior half. Prescutellar depression distinct, narrow medially and widened laterally, with several rather dense crenulation, 0.1 times as long as scutellum. Scutellum weakly convex, without lateral carinae, almost as long as maximum basal width. Subalar depression narrow, shallow, finely reticulate. Precoxal sulcus indistinct. Postpectal carina absent. Metanotum with low, wide and distally not acuminate medio-dorsal tubercle (lateral view). Metapleural flange (lobe) rather narrow, relatively long, subrounded distally. Propodeum almost straight in anterior half and weakly rounded in posterior half (lateral view), without lateral tubercles.

Wings. Fore wing 3.8 times longer than wide. Pterostigma wide, 3.0 times longer than its maximum width. Radial vein (r) arising before middle of pterostigma, from its basal 0.4. Radial (marginal) cell weakly shortened, metacarpus (1-R1) 1.5 times longer than pterostigma. Second radial abscissa (3SR) 4.8 times longer than first abscissa ( $\mathrm{r}$ ) and forming with it straight angle, 0.8 times as long as the evenly curved third abscissa (SR1), 2.1 times as long as straight first radiomedial vein (2-SR). Second radiomedial (submarginal) cell weakly narrowed distally, its length 3.2 times maximum width, 1.7 times length of brachial (subdiscal) cell. Second abscissa of medial vein $(2-\mathrm{SR}+\mathrm{M})$ long, 1.5 times longer than recurrent vein (m-cu). Nervulus (cu-a) weakly postfurcal and oblique towards base of wing. Mediocubital vein $(\mathrm{M}+\mathrm{CU} 1)$ distinctly sinuate and strongly curved medially to longitudinal anal vein (1-1A). Brachial (subdiscal) cell wide basally and narrowed towards apex, evenly closed apically on the level of recurrent vein (m-cu). Parallel vein (CU1a) interstitial. Hind wing 4.7 times longer than its maximum width. First costal abscissa $(\mathrm{C}+\mathrm{SC}+\mathrm{R}) 0.8$ times as long as second abscissa (1-SC+R). First abscissa of mediocubital vein $(\mathrm{M}+\mathrm{CU}) 0.55$ times as long as second and third abscissa $(1-\mathrm{M})$ combined. Recurrent vein $(\mathrm{m}-\mathrm{cu})$ long, unsclerotised and not pigmented, interstitial, distinctly and evenly curved towards base of wing.

LEgS. Fore tibia anterior margin with rather long and rather dense spines arranged in long single line. Segments of middle tarsus rather short, weakly longer than their width. Hind coxa rather short, without basoventral corner and tubercle, 1.4 times longer than maximum width. Hind femur weakly thickened, elongate-oval, 3.5 times longer than wide, with distinct antero-dorsal protuberance. Hind tibia with six slender spines on outer distal margin. Hind tarsus 0.85 times as long as hind tibia. Hind basitarsus 0.55 times as long as remaining segments combined. Second segment of hind tarsus 0.6 times as long as basitarsus, 1.1 times longer than fifth segment (without pretarsus).

Metasoma. Petiole in lateral view, ventrally weakly curved, dorsally strongly arched in anterior half and almost straight in posterior half, highest in anterior third; in dorsal view, petiole rather broad, weakly evenly widening towards anterior quarter (till distinct spiracular tubercles), and weakly widened to 
apex of petiole, with very fine dorsope. Length of petiole 2.3 times its posterior width, 1.6 times length of propodeum; posterior width almost equal to its width at level of spiracles, 1.5 times its minimum anterior width. Second to fifth tergites with laterotergites separated; second and third lateral parts of tergites not fused. Second suture present, but very shallow, weakly curved and reticulate with very fine crenulation partly. Median length of second tergite 0.9 times its anterior width, almost as long as third tergite. Median length of second and third tergites combined 1.7 times anterior width of second tergite, 0.7 times their maximum width. Ovipositor straight. Ovipositor sheath 1.2 times longer than petiole, 0.3 times as long as metasoma, 0.6 times as long as mesosoma, 0.3 times as long as fore wing.

SCULPTURE AND PUBESCENCE. Head densely granulate, without aciculation; face medially finely coriaceous in vertical stripe, temple below finely granulate-coriaceous. Mesoscutum densely and small reticulateareolate, without carinae and rugulosity medio-posteriorly; scutellum entirely finely reticulate. Mesoand metapleura entirely densely and finely reticulate-coriaceous. Propodeum only with basolateral areas delineated by rather distinct carinae, areola absent; propodeum almost smooth in basomedial fifth, then medially reticulate-rugulose; transverse and longitudinal striate with rugulosity in posterior third. Hind coxa almost entirely areolate-coriaceous; hind femur mainly very finely reticulate. Petiole mainly reticulate-rugulose, almost smooth medio-posteriorly, with short and subparallel dorsal carinae in anterior 0.4. Second to sixth tergites entirely and very densely small reticulate-areolate, sculpture becoming finer towards posterior; second suture without crenulation. Vertex mainly glabrous, with short and sparse yellow setae laterally. Mesoscutum widely glabrous, with short and sparse yellow setae arranged along trace of notauli and laterally. Setae of hind tibia semi-erect, short and rather sparse, their length on dorsal surface about 0.3 times maximum width of tibia.

Colour. Body mainly reddish brown to dark reddish brown, head mainly light reddish brown but infuscate dorsally. Palpi dark brown. Antenna mainly yellow to brownish yellow, two apical segments brown to dark brown. Legs mainly reddish brown with dark spots, fore and middle coxae light reddish brown; fore femur anteriorly partly brownish yellow; all tibiae almost black, tarsi yellow medially, dark basally and apically. Ovipositor sheath almost black. Fore wing strongly infuscate, paler distally, with hyaline vertical stripes wide basally, narrow on level of maximum bend of mediocubital vein $(\mathrm{M}+\mathrm{CU})$ and under base of pterostigma. Pterostigma mainly black, white in basal 0.4.

\section{Male}

Body length $4.3 \mathrm{~mm}$; fore wing length $2.2 \mathrm{~mm}$. Antenna 22-segmented, with three dark apical segments. Penultimate segment 2.4 times longer than width. Mesosoma maximum length 1.7 times its maximum height. Hind femur 3.1 times longer than wide. Otherwise very similar to female.

\section{Distribution}

Yemen.

Spathius nixoni Belokobylskij \& Maetô, 2009

Figs 22-23

Spathius nixoni Belokobylskij \& Maetô, 2009: 654.

Spathius nixoni - Yu et al. 2016.

\section{Material examined}

UNITED ARAB EMIRATES • 7 우; "United Arab Emirates, Bithnah (3699), at light, 11.xii.2005 -

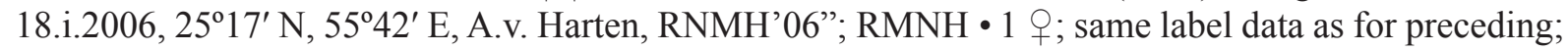
ZISP • 1 क; "United Arab Emirates, Wadi Shawkah (6179-6193), water traps, 31.x-27.xi.2006, 


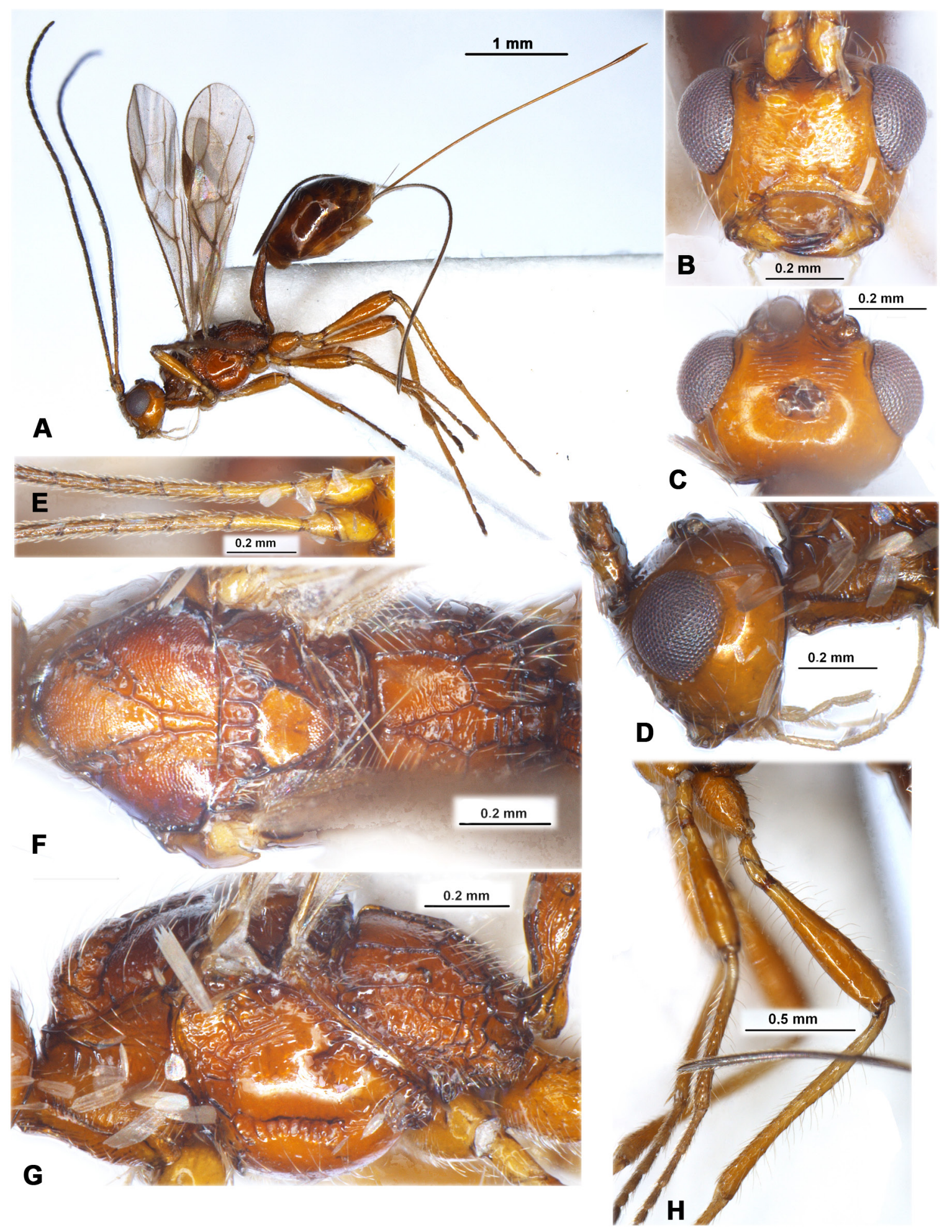

Fig. 22. Spathius nixoni Belokobylskij \& Maetô, 2009, q (RMNH). A. Habitus, lateral view. B. Head, front view. C. Head, dorsal view. D. Head, lateral view. E. Basal segments of antenna. F. Mesosoma, dorsal view. G. Mesosoma, lateral view. H. Hind leg. 


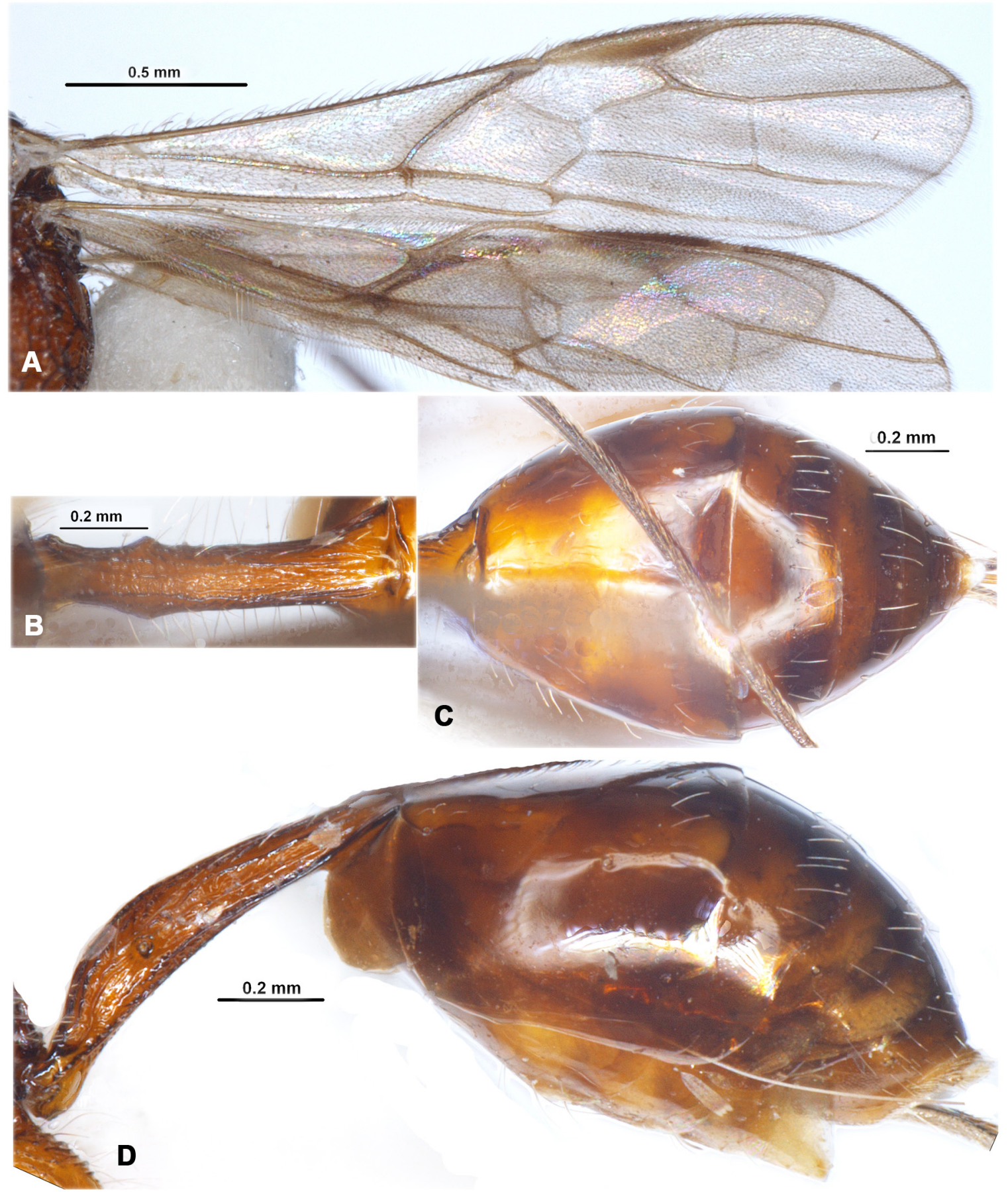

Fig. 23. Spathius nixoni Belokobylskij \& Maetô, 2009, $q$ (RMNH). A. Wings. B. Petiole of metasoma, dorsal view. C. Metasoma without petiole, dorsal view. D. Metasoma, lateral view. 
A.v. Harten, RNMH'06”; RMNH • 1 ภ̀; “United Arab Emirates, Hatta (6402-6403), at light, 24-30. vi.2006, 244' N, 560' E, A.v. Harten, RNMH'06”; RMNH.

YEMEN • 2 + 9 ; “Yemen (no 2910), Ta'izz, light trap, 5.i-2.ii.1998, A. van Harten, RMNH'98”; RMNH - 6 우; "Yemen (3540), Ta'izz, 3-24.i.1999, light-trap, A.v. Harten \& M. Mahyoub, RMNH'00"; RMNH • 3 क $O$; "Yemen, Ta'izz, viii.1999, light tr., no 4056, A.v. Harten \& A. Awad, RMNH'01"; RMNH • 1 क; same label data as for preceding; ZISP • 3 qo+; "Yemen (4056), Ta'izz, viii.1999, lighttrap, A.v. Harten \& A. Awad, RMNH'00"; RMNH • 1 o; "Yemen (4057), Ta'izz, ix.1999, light-trap, A.v. Harten \& A. Awad, RMNH'00"; RMNH • 2 o ; ; "Yemen, Ta'izz, ix.2000, light tr., no 4927 A.v. Harten \& A.R. Al Yarimi, RMNH'01"; RMNH • 12 o 9 ; "Yemen, Ta'izz, x.1999, light tr., no 4249, A.v. Harten \& A. Awad, RMNH'01"; RMNH • 1 क; same label data as for preceding; ZISP • 1 ; "Yemen, Ta'izz, xi.1999, light tr., no 4138, A.v. Harten \& A. Awad, RMNH'01"; RMNH • 10 o $O$; "Yemen (6090), Al Kadan, x.2001, light trap; A.v. Harten \& T. Abdul-Haq, RMNH’03"; RMNH • 2 우; same label data as for preceding; ZISP • 1 ऊ’; "Yemen (no 2893), Al Kadan (20 km N Bajil), Mal. trap, 3.xii.1997 - 16.ii.1998, A.v. Harten, RMNH'98"; RMNH • 1 क; "Yemen (6158), Al Lahima, 17.ix-14. ii.2001, Malaise trap, A.v. Harten, RMNH'02"; RMNH • 1 ㅇ "Yemen (5486), Al Lahima, 1.i-9.iv.2001, Mal. trap, A.v. Harten, RMNH'02"; RMNH.

\section{Distribution}

Japan, *UAE, *Yemen.

\section{Remarks}

This species was originally described from Japan and perhaps also occurs in China (Belokobylskij \& Maetô 2009). The record of this species from the Arabian Peninsula strongly widens its known geographic distribution, and it is likely to also occur in the intervening part of Asia. Specimens from Yemen and the UAE possess a distinctive dark body colour.

Spathius subafricanus Belokobylskij sp. nov. urn:1sid:zoobank.org:act:94C0551F-AF56-4B11-BD40-BB5A98F4042E

Figs 24-25

\section{Comparative diagnosis}

According to the dense aciculate sculpture of the face, widely smooth mesopleuron, mediocubital $(\mathrm{M}+\mathrm{CU} 1)$ vein of the fore wing weakly sinuate and hind coxa with a basoventral tubercle, S. subafricanus sp. nov. belongs to the S. vulnificus Wilkinson species-group. This new species is morphologically similar to $S$. paracritolaus Belokobylskij, 1996 from China, but differs from it by having the first flagellar segment of the antenna 4.5 times longer than its apical width (5.5 times in S. paracritolaus), head behind eyes almost parallel in the anterior half and roundly narrowed in the posterior half (evenly roundly narrowed in $S$. paracritolaus), pronotal keel weakly separated from the posterior margin of pronotum (fused medially in $S$. paracritolaus), mesopleuron widely smooth submedially (entirely and densely reticulate in $S$. paracritolaus), metanotum (lateral view) mediodorsally with a short pointed tubercle (with obtuse tubercle in S. paracritolaus), precoxal sulcus deep (shallow in S. paracritolaus), nervulus (cu-a) of fore wing distinctly postfurcal (interstitial in S. paracritolaus), petiole of metasoma 1.7 times longer than posterior width (2.0 times in $S$. paracritolaus), sculpture on fourth and fifth metasomal tergites absent (present anteriorly in $S$. paracritolaus), second tergite with separated laterotergites (not separated in S. paracritolaus), ovipositor 2.5 times longer than petiole and 0.6 times as long as fore wing (1.5 and 0.4 times respectively in $S$. paracritolaus), and hind tibia uniformly yellow, without dark spots (with dark submedial spot in $S$. paracritolaus). 
Spathius subafricanus sp. nov. is also similar to S. aciculatus Tang, Belokobyskij \& Chen, 2015 from China (Hainan), but differs from it in having the first flagellar segment of the antenna 4.5 times longer than its apical width (5.5 times in S. aciculatus), transverse diameter of the eye 1.6 times length of temple (1.9 times in $S$. aciculatus), vertex smooth laterally and in posterior half (entirely densely aciculate in $S$. aciculatus), pronotal keel weakly separated from the posterior margin of the pronotum (widely fused medially in S. aciculatus), notauli deep in the posterior half (very shallow in $S$. aciculatus), mesopleuron widely smooth submedially (entirely and weakly rugulose in S. aciculatus), and petiole short, its length 1.7 times its posterior width (dorsal view) and 1.3 times the length of the propodeum (3.0 and 0.9 times accordinctly in $S$. aciculatus).

\section{Etymology}

This species is named after Africa, because its type material was collected from a territory near this continent.

\section{Material examined}

Holotype

UNITED ARAB EMIRATES • +; "United Arab Emirates, Fujairah (1314), light tr., 13-19.iv.2005, $25^{\circ} 08^{\prime} \mathrm{N}, 56^{\circ} 21^{\prime}$ E. A.v. Harten, RMNH’05”; RMNH.

\section{Paratypes}

UNITED ARAB EMIRATES • 1 क; "United Arab Emirates, Fujairah (4117), light tr., 28.ii-1.iv.2006, $25^{\circ} 08^{\prime} \mathrm{N}, 56^{\circ} 21^{\prime}$ E, A.v. Harten, RNMH'06”; RMNH 1 1 ; same label data as for preceding; ZISP.

\section{Description}

\section{Female}

Measurements. Body length 3.6-4.4 mm; fore wing length 2.5-2.7 mm.

HEAD. Head width (dorsal view) 1.3-1.4 times its median length, 1.2 times width of mesoscutum. Vertex convex. Head behind eyes (dorsal view) subparallel in anterior half and evenly roundness decreased in posterior half. Transverse diameter of eye (dorsal view) 1.4-1.5 times longer than temple. Ocelli small, in triangle with base 1.3-1.4 times its sides. POL almost equal to Od, 0.45-0.50 times OOL. Eye glabrous, in lateral view weakly oblique, its maximum diameter 1.3 times minimum diameter. Malar space 0.5-0.6 times maximum diameter of eye, 1.0-1.2 times basal width of mandible. Face weakly convex, its width 1.1 times maximum diameter of eye, 1.0-1.1 times height of face and clypeus combined. Clypeal suture mainly absent, distinct laterally at short lower part. Ventral margin of clypeus with distinct narrow flange. Hypoclypeal depression medium-sized and oval, its width 0.7-0.9 times shortest distance from edge to eye, 0.4 times width of face. Occipital carina dorsally complete and not broken toward ocellar triangle, not reaching below hypostomal carina and obliterated relatively short distance before mandible base. Hypostomal flange narrow.

AntenNa. Antenna slender, weakly setiform, 28-29-segmented, almost as long as body. Scape 1.5-1.7 times longer than its maximum width. First flagellar segment 4.2-4.3 times longer than apical width, 1.00-1.05 times as long as second segment. Penultimate segment 3.0-3.5 times longer than width, 0.5 times as long as first segment, 0.9 times as long as apical segment; latter not acuminate apically and without spine.

Mesosoma. Mesosoma not depressed, its maximum length 1.9-2.0 times its maximum height. Pronotal carina fine, its anterior branch situated submedially and separated from posterior margin of pronotum. Pronotal lateral depression distinct, wide, crenulate, delineated upper and below by carinae. Mesoscutum (lateral view) rather high, subroundly and curvedly elevated above pronotum, its median lobe (dorsal 

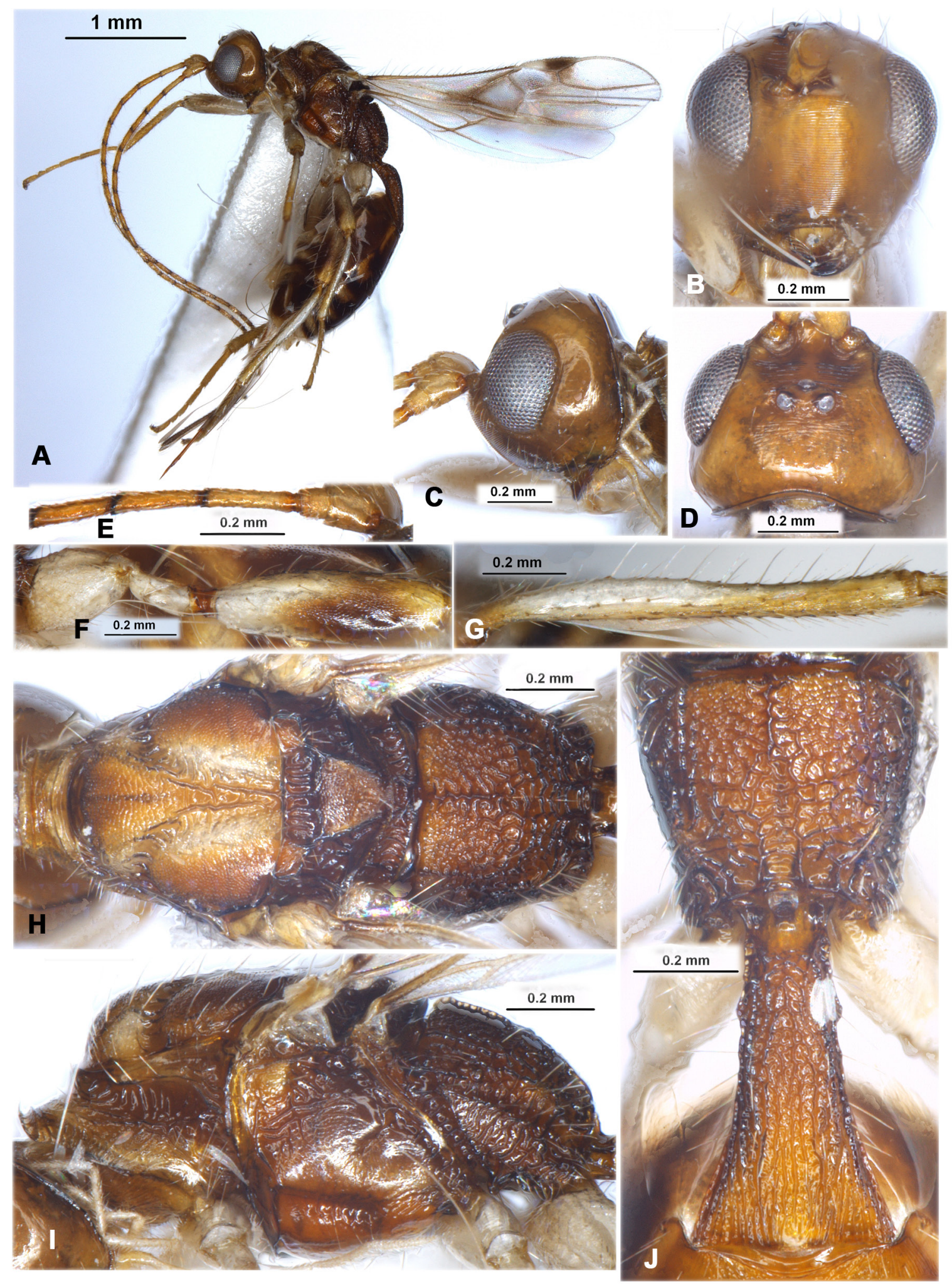

Fig. 24. Spathius subafricanus Belokobylskij sp. nov., holotype, o (RMNH). A. Habitus, lateral view. B. Head, front view. C. Head, lateral view. D. Head, dorsal view. E. Basal segments of antenna. F. Hind coxa and femur. G. Hind tibia. H. Mesosoma, dorsal view. I. Mesosoma, lateral view. J. Propodeum and petiole of metasoma, dorsal view. 

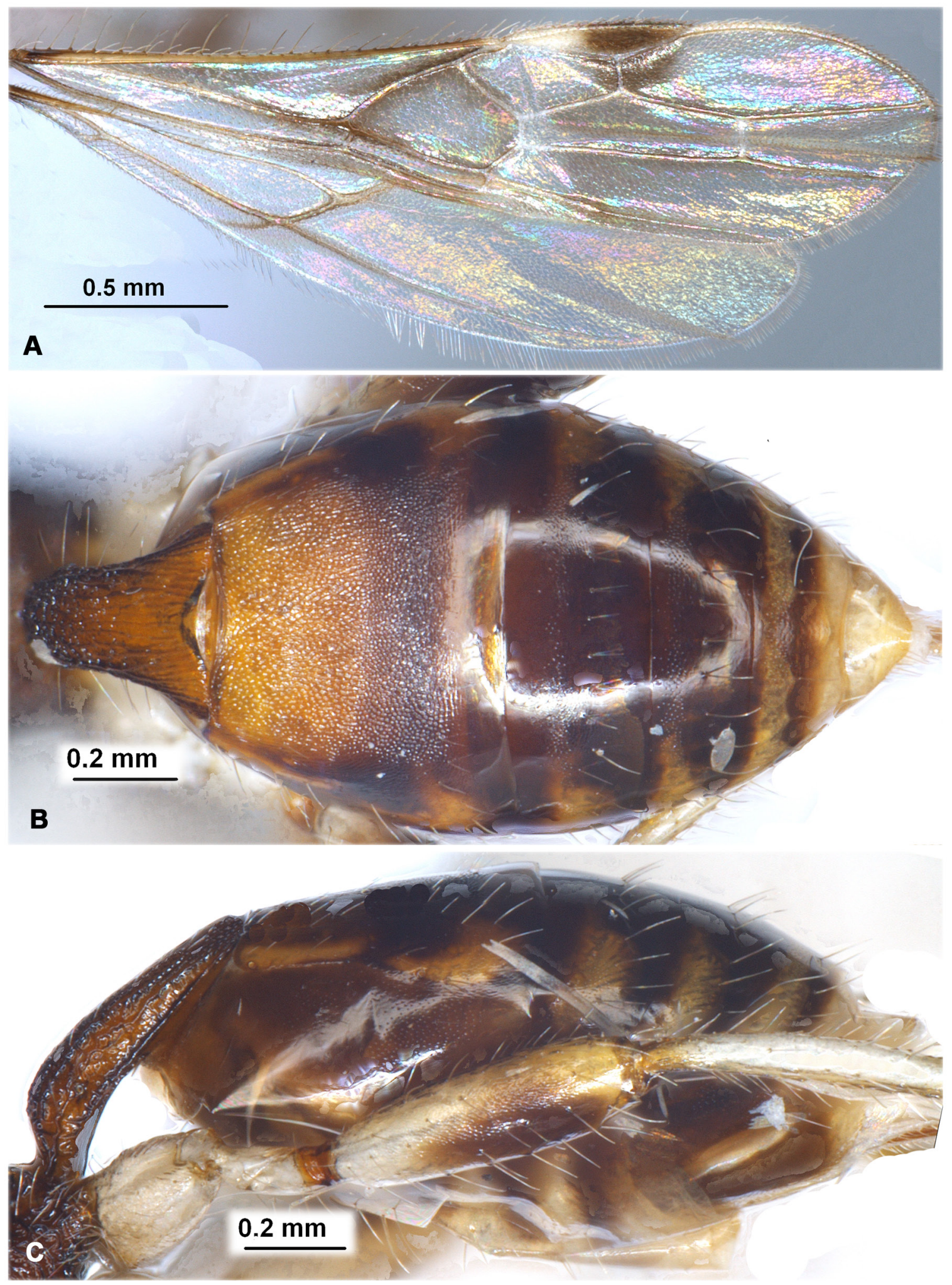

Fig. 25. Spathius subafricanus Belokobylskij sp. nov., holotype, $q$ (RMNH). A. Wings. B. Metasoma, dorsal view. C. Metasoma, lateral view. 
view) distinctly convex anteriorly and without anterolateral corners, without longitudinal median furrow; mesoscutum in dorsal view almost as long as wide. Notauli complete, wide, deep anteriorly and shallow posteriorly, densely crenulate. Prescutellar depression rather deep, wide, with six-seven carinae, 0.4 times as long as scutellum. Scutellum weakly convex, with distinct lateral carinae, almost as long as maximum anterior width. Subalar depression wide, shallow, sparsely rugulose-striate. Precoxal sulcus deep, wide, short, densely crenulate, running along anterior 0.5 of lower part of mesopleuron. Postpectal carina absent. Metanotum with low, wide below and distally weakly acuminate medio-dorsal tubercle (lateral view). Metapleural flange (lobe) narrow, distinct, subrounded distally. Propodeum evenly roundly convex, without lateral tubercles.

Wings. Fore wing 3.8-4.1 times longer than wide. Pterostigma rather wide, 4.0-4.4 times longer than its maximum width. Radial vein (r) arising weakly behind middle of pterostigma. Radial (marginal) cell not shortened, metacarpus (1-R1) 1.2 times longer than pterostigma. Second radial abscissa (3-SR) 3.3-3.7 times longer than first abscissa (r) and forming with it weakly obtuse angle, 0.6-0.7 times as long as straight third abscissa (SR1), 1.1 times longer than straight first radiomedial vein(2-SR). Second radiomedial (submarginal) cell weakly narrowed distally, its length 3.2-3.5 times maximum width, 1.4-1.6 times length of brachial (subdiscal) cell. Second abscissa of medial vein (2-SR+M) rather long, $0.7-0.8$ times as long as recurrent vein $(\mathrm{m}-\mathrm{cu})$. Nervulus (cu-a) postfurcal, 1.5-1.6 times longer than distance between basal (1-M) vein and nervulus (cu-a). Mediocubital vein (M+CU1) weakly sinuate to longitudinal anal vein (1-1A). Narrow brachial (subdiscal) cell closed apically weakly behind recurrent vein (m-cu). Parallel vein (CU1a) not interstitial, arising from anterior third of the vein closed brachial (subdiscal) cell distally. Hind wing 5.0-5.2 times longer than its maximum width. First costal abscissa $(\mathrm{C}+\mathrm{SC}+\mathrm{R}) 0.5$ times as long as second abscissa $(1-\mathrm{SC}+\mathrm{R})$. First abscissa of mediocubital vein $(\mathrm{M}+\mathrm{CU})$ $0.55-0.60$ times as long as second and third abscissa (1-M) combined. Recurrent vein (m-cu) rather short, unsclerotised but pigmented, antefurcal, curved towards base of wing.

LEGS. Fore tibia anterior margin with short and dense spines arranged in long single vertical line. Segments of middle tarsus distinctly longer than their width. Hind coxa rather short, with basoventral corner and small tubercle, 1.3-1.4 times longer than its maximum width. Hind femur thickened, elongate-oval, 3.2-3.3 times longer than wide. Hind tibia with several slender spines on outer distal margin. Hind tarsus 0.95 times as long as hind tibia. Hind basitarsus 0.65 times as long as remaining segments combined. Second segment of hind tarsus $0.45-0.50$ times as long as basitarsus, 1.2-1.4 times longer than fifth segment (without pretarsus). Tarsal segments rather slender.

Metasoma. Petiole in lateral view, ventrally weakly evenly curved, dorsally distinctly arched in anterior third and almost straight on remaining part, highest in anterior fourth; in dorsal view, petiole distinctly evenly widening from base towards its apex, with weak spiracular tubercles in anterior quarter and fine dorsope. Length of petiole 1.7 times its posterior width, 1.5 times length of propodeum; posterior width 1.7-1.8 times its width at spiracular level, 2.4-2.5 times its minimum anterior width. Second tergite with separated laterotergites; following tergites without separated laterotergites; laterotergites of second and third tergites fused, without suture. Second suture almost absent. Median length of second and third tergites combined 1.3 times anterior width of second tergite, $0.7-0.8$ times their maximum width. Ovipositor straight. Ovipositor sheath 2.0-2.6 times longer than petiole, $0.6-0.8$ times as long as metasoma, $0.95-1.20$ times longer than mesosoma, $0.50-0.65$ times as long as fore wing.

SCULPTURE AND PUBESCENCE. Vertex finely transversely striate in median anterior half, smooth laterally and posteriorly; frons distinctly and densely transversely striate; face entirely with very dense and thin aciculation; temple entirely smooth. Mesoscutum and scutellum with very dense and fine granulation; mesoscutum additionally with two distinctly subparallel median carinae in posterior half and rugulosity between carinae. Mesopleuron widely smooth, with fine reticulation of striation laterally, upper and 
lower. Propodeum entirely rugose-areolate, with fine granulation anteriorly, with areas rather distinctly delineated by carinae, medio-basal carina in anterior third of propodeum about 1.5 times as long as anterior fork of areola; areola long and narrow, 2.5 times longer than its maximum width. Hind coxa transverse striate dorsally, rugulose-striate with granulation laterally; hind femur fine reticulate-coriaceous, with rugulosity dorsally, almost smooth below. Petiole distinctly and densely striate, with dense rugosity in anterior half. Second and third tergites densely and small reticulate- areolate in wide median areas, smooth posteriorly and laterally. Remainder tergites almost entirely smooth. Vertex mainly with long and very sparse pale setae. Mesoscutum with long and sparse white setae arranged widely along notauli and laterally, glabrous on wide median areas on lobes. Setae of hind tibia semi-erect, medium length and rather dense, their length on dorsal surface $0.9-1.3$ times maximum width of tibia.

Colour. Head and anterior half of mesosoma light reddish brown with yellowish areas, posterior half of mesosoma and metasoma reddish brown, metasoma laterally with yellowish spots or stripes. Palpi pale yellow. Antenna mainly brownish yellow, infuscate apically on one-two segments. Legs mainly brownish yellow to yellow, all femora with additional elongate dark spots dorsally or ventrally. Ovipositor sheath pale yellow in anterior half, brown to dark brown in posterior half. Fore wing mainly finely infuscate, with several hyaline spots. Pterostigma dark brown in apical half, whitish in basal half.

\section{Male}

Unknown.

\section{Distribution}

UAE, Yemen.

Tribe Hecabolini Foerster, 1863

Genus Hecabalodes Wilkinson, 1929

\section{Type species}

Hecabalodes anthaxiae Wilkinson, 1929, by monotypy.

\section{Remarks}

Hecabalodes is a small genus that is very similar to Leluthia. The main difference between these two genera is only in the distinctly shortened radial (marginal) cell of the fore wing, which is present in Hecabalodes. However, the shortened radial (marginal) cell also occurs in some species of Leluthia (e.g., L. ruguloscolyti (Fischer, 1962) has an intermmediate-sized cell).

\section{Distribution}

Afrotropical, Oriental and Palaearctic regions.

\section{Key to species of the genus Hecabalodes}

1. Recurrent vein of fore wing (m-cu) postfurcal (Figs 27A, 33A). Radial (marginal) cell of fore wing long; its metacarpus (1-R1) 1.3-1.5 times longer than distance from apex of radial (marginal) cell to apex of wing (Figs 27A, 33A). Second radial abscissa (3-SR +SR1) of fore wing always almost straight (Figs 27A, 33A). African species

- Recurrent vein of fore wing (m-cu) antefurcal or sometimes interstitial (Figs 29A, 31A). Radial (marginal) cell of fore wing short; its metacarpus (1-R1) not longer than distance from apex of radial (marginal) cell to apex of wing. Second radial abscissa (3-SR +SR1) of fore wing aften more or less evenly curved, but sometimes almost straight (Figs 29A, 31A). Asian species 
2. Vertex densely undulately transversely striate with rugulosity between striae (Fig. 26B). First metasomal tergite longer, lorger than its posterior width (Fig. 27B). Ovipositor sheath longer than metasoma (Fig. 26A). Body length 3.0-6.0 mm. - Sudan, UAE, Yemen

H. anthaxiae Wilkinson, 1929

- Vertex only densely and distinctly or sometimes finely granulate, without striae (Fig. 32B). First metasomal tergite shorter than its posterior width (Fig. 33B). Ovipositor sheath shorter than metasoma (Fig. 32A). Body length 2.3-4.4 mm. - Algeria, Chad, Mauritania, UAE, Yemen

H. xylophagi Fischer, 1962

3. Vertex densely and coarsely transversely undulately striate with reticulation (Fig. 28D). Notauli complete and rather distinct posteriorly (Fig. 28G). Second radial abscissa (3-SR +SR1) of fore wing always distinctly evenly curved (Fig. 29A). Metacarpus (1-R1) distinctly shorter than distance from apex of radial (marginal) cell to apex of wing (Fig. 29A). Medial (basal) cell of fore wing almost glabrous or partly with very short setae (Fig. 29A). Body length 3.3-4.3 mm. UAE

H. maculatus Belokobylskij sp. nov.

- Vertex only densely granulate, without striation (Fig. 30C). Notauli incomplete, almost absent posteriorly (fused with medio-posterior rugose area) (Fig. 30E). Second radial abscissa (3-SR +SR1) of for wing almost straight or weakly and not evenly curved (Fig. 31A). Metacarpus (1-R1) almost equal to distance from apex of radial (marginal) cell to apex of wing (Fig. 31A). Medial (basal) cell of fore wing almost rather distinctly setose by medium length setae (Fig. 31A)

4. Temple shorter, transverse diameter of eye 1.8-2.2 times longer than temple (Fig. 30C). Ovipositor sheath shorter than metasoma (Fig. 30A). Body length 2.2-4.0 mm. - Greece, India, Iran, Tajikistan, Turkey, Turkmenistan, UAE, Yemen xylophagi

H. radialis Tobias, 1962

- Temple longer, transverse diameter of eye 1.3-1.5 times longer than temple. Ovipositor sheath longer than metasoma. Body length 2.9-4.7 mm. - Tajikistan, Turkmenistan

H. tadzhicus Tobias, 1964

Hecabalodes anthaxiae Wilkinson, 1929

Figs 26-27

Hecabalodes anthaxiae Wilkinson, 1929: 106.

Hecabalodes anthaxiae - Fischer 1962: 298. — Yu et al. 2016. — Edmardash et al. 2020: 50.

\section{Material examined}

\section{Holotype}

SUDAN • 1 +; "Type", "B.M. Type Hym. 3. c.195", Hecabalodes anthaxiae Wilkinson. Type", "Khartoum, H.W. Badford, Dec., 1927, from branch Acacia Arabica infested with Buprestid sp. Sudan Govt.", "Africa, 1/250,000 55 B Map", "Ent. Coll. C 3853", "Hecabalodes anthaxiae Wilk. C. van Achterberg 1979, Lectotype"; BMNH.

\section{Additional material}

UNITED ARAB EMIRATES • 1 q; "United Arab Emirates, Wadi Safad (5020), at light, 14-21.v.2006, $25^{\circ} 13^{\prime} \mathrm{N}, 56^{\circ} 19^{\prime}$ E, A.v. Harten, RNMH'06”; RMNH • 1 \%; same label data as for preceding; ZISP •

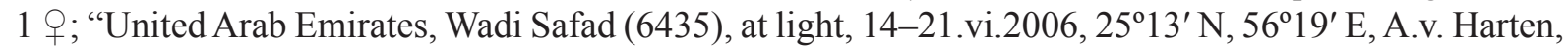
RNMH'06"; RMNH • 1 क; "United Arab Emirates, Fujairah (1587), hand coll., 2-13.v.2005, 2508' N, 56 21' E, A.v. Harten, RMNH’05”; RMNH - 1 q; "United Arab Emirates, Fujairah (1314), light tr., 13-19.iv.2005, $25^{\circ} 08^{\prime} \mathrm{N}, 56^{\circ} 21^{\prime}$ E. A.v. Harten, RMNH’05”; RMNH. 


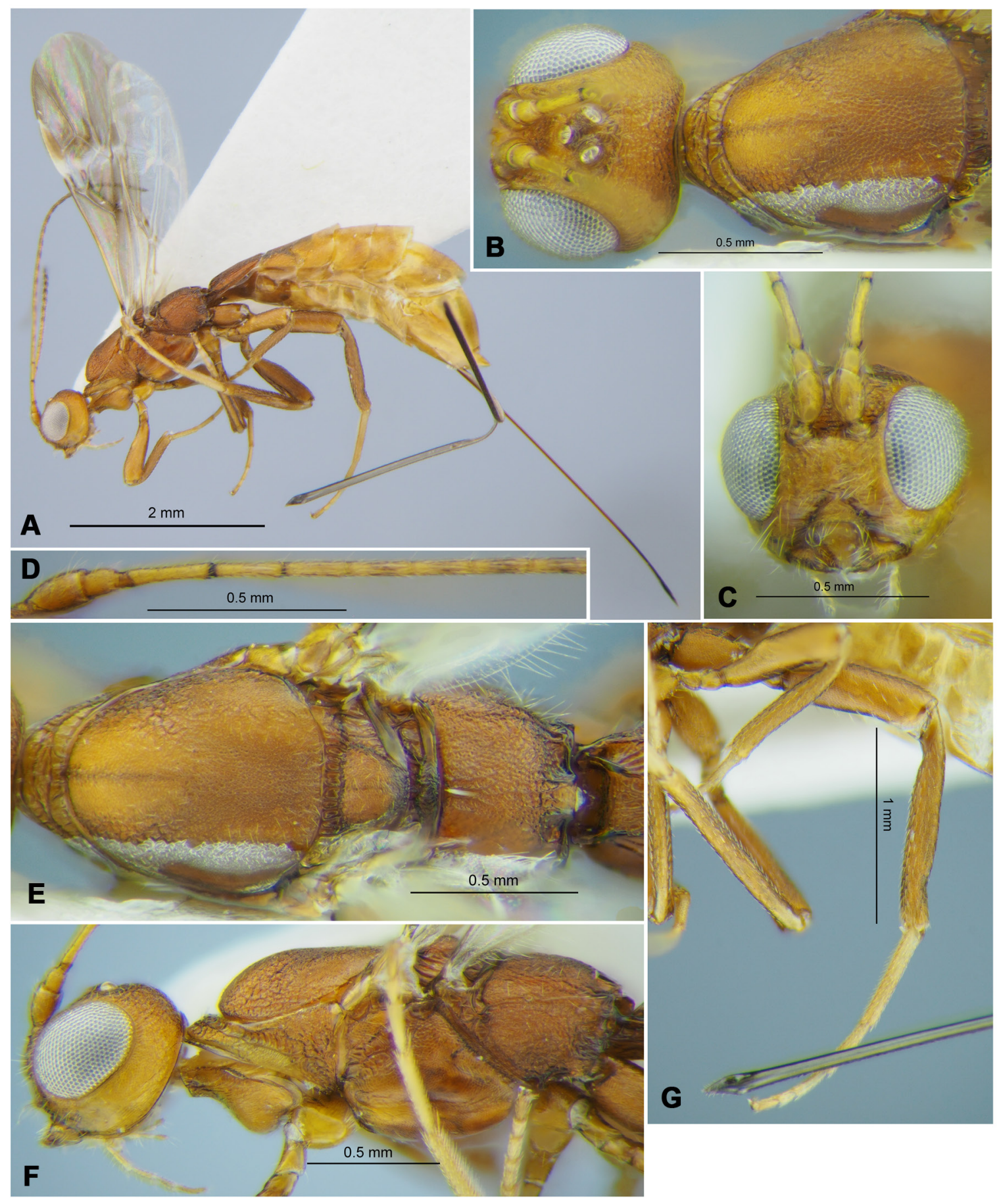

Fig. 26. Hecabalodes anthaxiae Wilkinson, 1929, $q$ (RMNH). A. Habitus, lateral view. B. Head and mesoscutum, dorsal view. C. Head, front view. D. Basal segments of antenna. E. Mesosoma, dorsal view. F. Head and mesosoma, lateral view. G. Hind leg. 

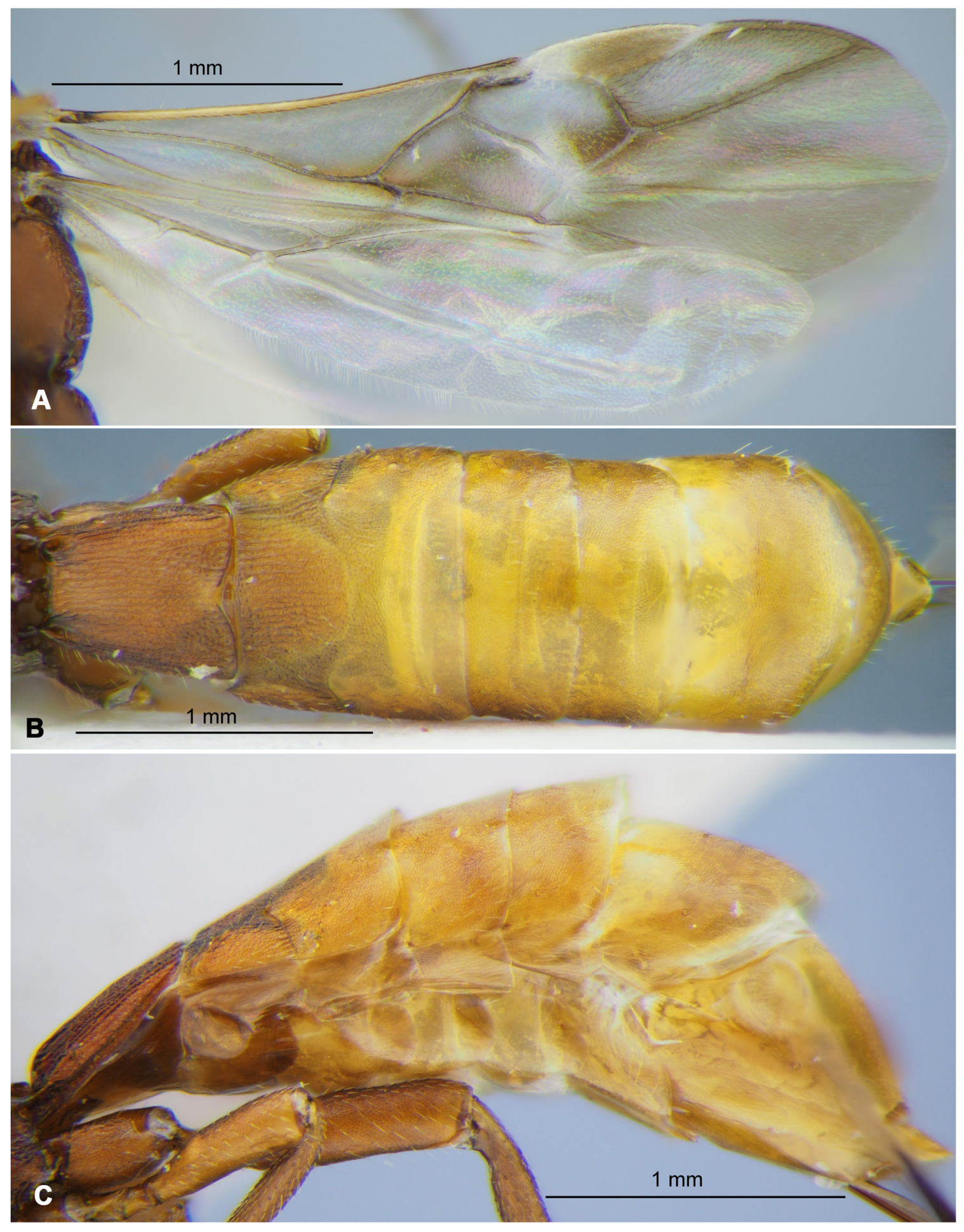

Fig. 27. Hecabalodes anthaxiae Wilkinson, 1929, $q$ (RMNH). A. Wings. B. Metasoma, dorsal view. C. Metasoma, lateral view. 
YEMEN • 3 क 9 ; “Yemen (8136), Al Kowd, ix.2003, light trap, A.v. Harten, S. Al Haruri, RMNH’03”; RMNH - 1 क; "Yemen (6117), Al Kowd, 21-28.viii.2001, Light trap, A.v. Harten, S. Al Haruri, RMNH'02"; RMNH • 6 우, 1 ð’; "Yemen, Al Kowd, v-vi.2000, light-tr., no 4719, A.v. Harten \& S. Al Haruri, RMNH'01"; RMNH • 1 ; ; same label data as for preceding; ZISP・ 1 +, 1 ; ; "Yemen (5750), Al Kowd, 8-12.vii.2001, light trap, A.v. Harten \& S. Al Haruri. RMNH'02”; RMNH・ 1 万; same label data as for preceding; ZISP • 1 q; "Yemen, Al Kowd, ii.2000, light tr., no 4431, A.v. Harten \& S. Al Haruri, RMNH'01"; RMNH • 1 क; "Yemen (5404), Hammam 'Ali, from coffee-berries (with Ceratitis capitata ?), 14.iii.2001, A.v. Harten, RMNH'02"; RMNH • 2 우; "Yemen (3901), Al Kowd, vii.1999, light-trap, A.v. Harten \& S. Al Haruri. RMNH'00"; RMNH • 1 क , 1 ठ; "Yemen (4054), Al Kowd, viii.1999, lighttrap, A.v. Harten \& S. Al Haruri, RMNH'00"; RMNH • 1 đ'; same label data as for preceding; ZISP • 1 †; "Yemen (4055), Al Kowd, ix.1999, light-trap, A.v. Harten \& S. Al Haruri, RMNH'00"; RMNH.

\section{Distribution}

Saudi Arabia, Sudan, *UAE, *Yemen.

Hecabalodes maculatus Belokobylskij sp. nov. urn:1sid:zoobank.org:act:BDFDB8D0-4AC5-4FEF-97AE-19B8AC977E55

Figs 28-29

\section{Comparative diagnosis}

The new species is similar to Hecabalodes tadzhicus Tobias, 1964 from Tajikistan and *Turkmenistan (1 q, Turkmenistan, Kugitang-tau Ridge [= Koytendag], Khodzhaifilata [near Shalkan Village], 19.v.1982, Belokobylskij [leg.]; ZISP), but differs from it in having the vertex densely and coarsely transversely undulately striate with reticulation (only densely granulate in H. tadzhicus), notauli complete and rather distinct (almost absent posteriorly in H. tadzhicus), precoxal sulcus mainly very shallow (entirely rather deep in H. tadzhicus), second radial abscissa (3-SR) of fore wing distinctly evenly curved (almost straight or weakly and not evenly curved in H. tadzhicus), metacarpus (1-R1) distinctly shorter than distance from apex of radial (marginal) cell to apex of wing (almost equal to it in H. tadzhicus), and medial (basal) cell of fore wing almost glabrous or partly with very short setae (rather distinctly setose by medium length setae in H. tadzhicus).

\section{Etymology}

From the Latin word 'maculatus' ('spotted'), because the fore wing in this species has numerous dark spots.

\section{Material examined}

\section{Holotype}

UNITED ARAB EMIRATES - O; "United Arab Emirates, NARC near Sweihan (1410), light trap, 1.ii-14.iii.2005, $24^{\circ} 2^{\prime} \mathrm{N}, 5^{\circ} 2^{\prime}$ E, A.v. Harten, RNMH'05”; RMNH.

\section{Paratypes}

UNITED ARAB EMIRATES • 1 \%; "United Arab Emirates, NARC near Sweihan (1299), light trap, 9-20.iv.2005, $24^{\circ} 2^{\prime}$ N, 55 $2^{\prime}$ E, A.v. Harten, RNMH'06”; RMNH • 1 ; ; same label data as for preceding; ZISP• 1 \& (without metasoma); "United Arab Emirates, NARC near Sweihan (4387), light trap, 26.ii-2.

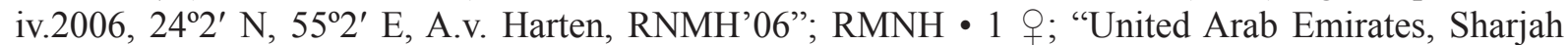

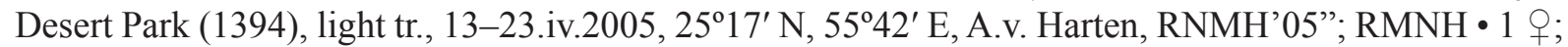
"United Arab Emirates, Al-Ajban (5937), Malaise tr., 27.v-26.vi.2006, 24³6' N, 5501' E, A.v. Harten, RNMH'06”; RMNH • 1 क; "United Arab Emirates, Al-Ajban (6418), Malaise \& light tr., 7-28.xii.2006, $24^{\circ} 3^{\prime} \mathrm{N}, 55^{\circ} 0^{\prime}$ E, A.v. Harten, RNMH'07"; RMNH • 1 \%; "United Arab Emirates, SSW of ad-Dhaid

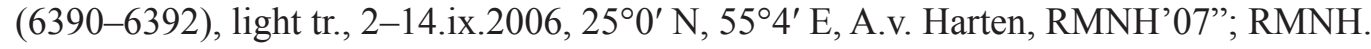




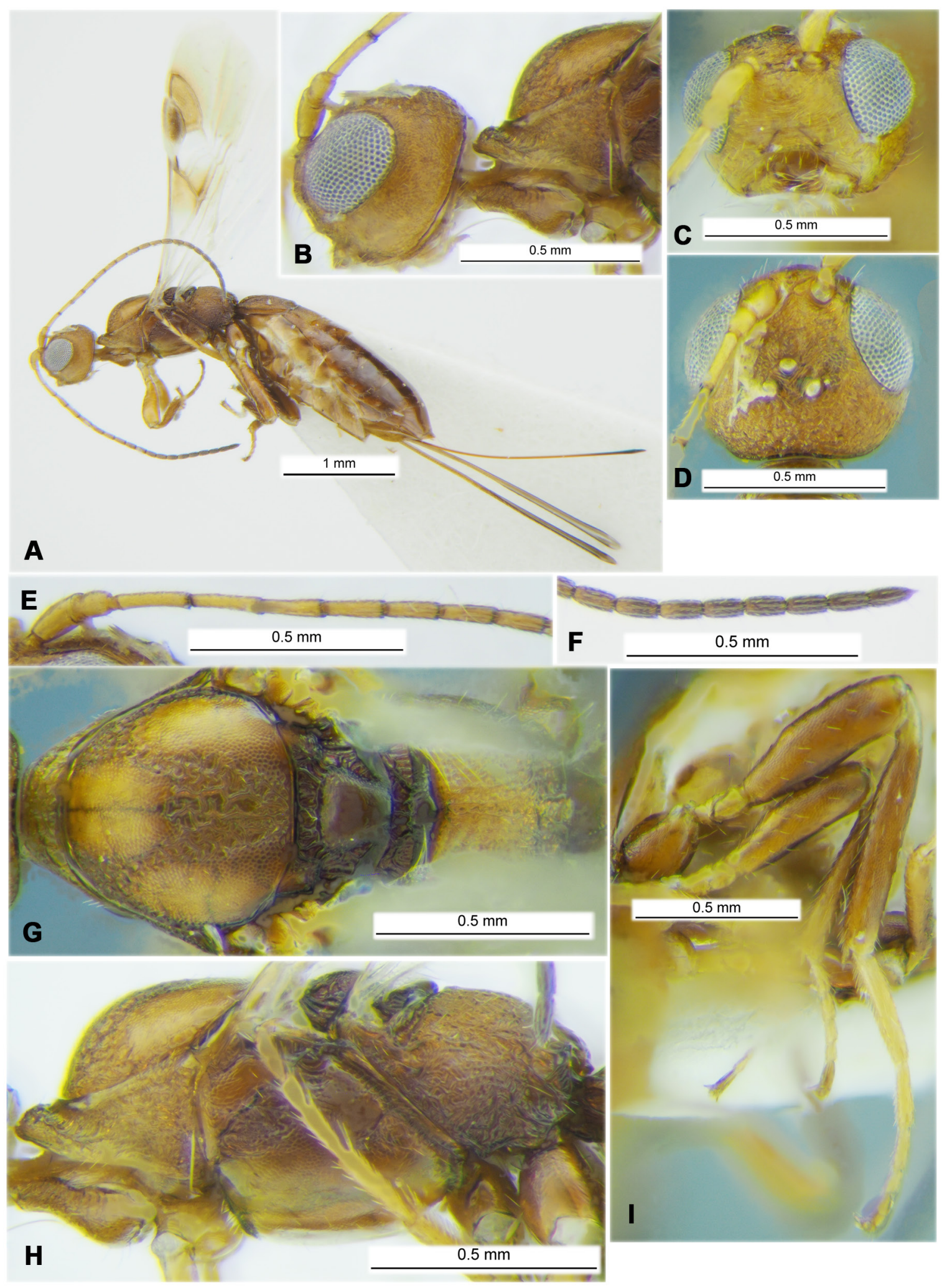

Fig. 28. Hecabalodes maculatus Belokobylskij sp. nov., holotype, $q$ (RMNH). A. Habitus, lateral view. B. Head and anterior part of mesosoma, lateral view. C. Head, front view. D. Head, dorsal view. E. Basal segments of antenna. F. Apical segments of antenna. G. Mesosoma, dorsal view. H. Head and mesosoma, lateral view. I. Hind leg. 

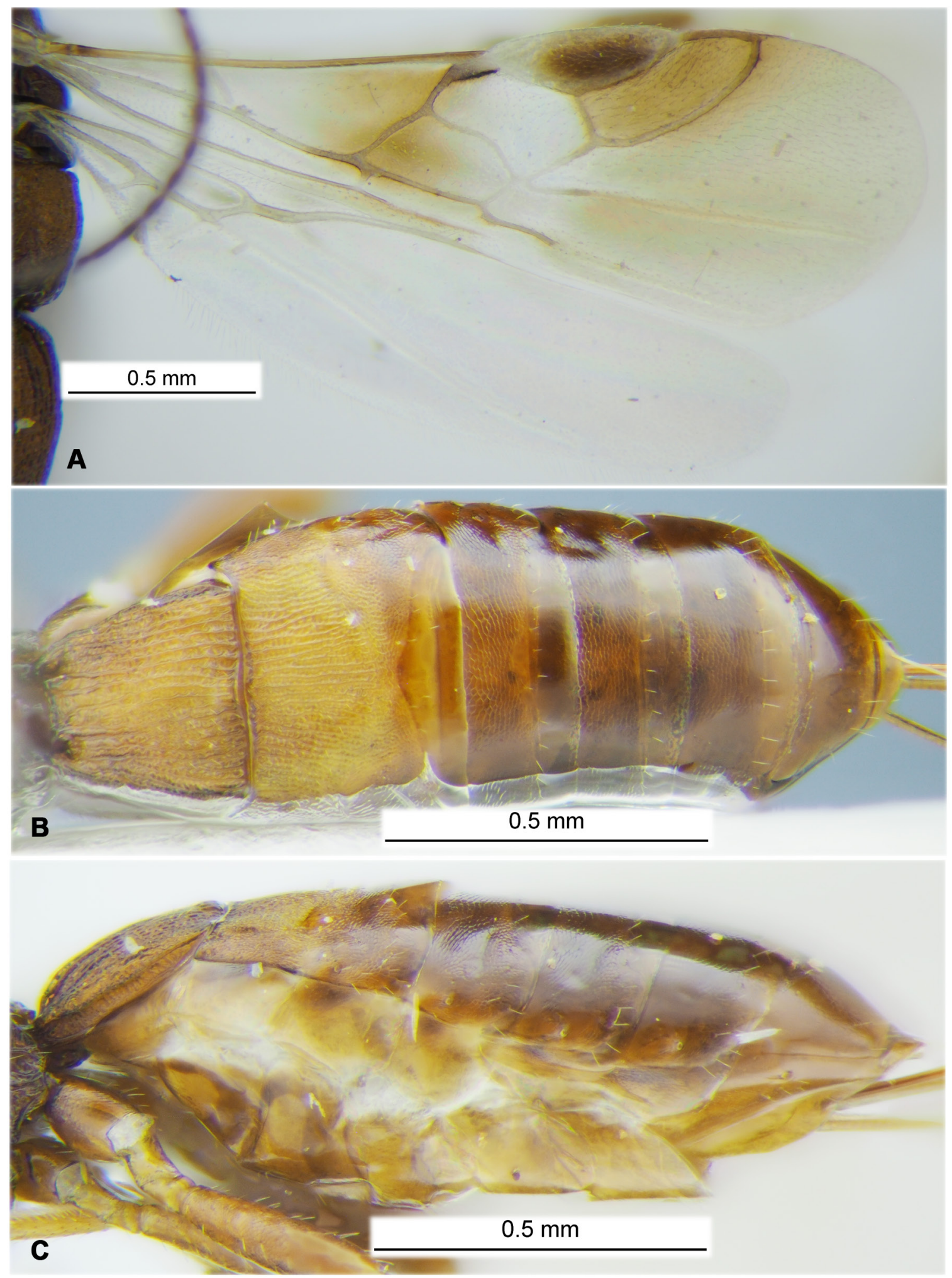

Fig. 29. Hecabalodes maculatus Belokobylskij sp. nov., holotype, $q$ (RMNH). A. Wings. B. Metasoma, dorsal view. C. Metasoma, lateral view. 


\section{Description}

Female

Measurements. Body length 3.3-4.3 mm; fore wing length $2.0-2.4 \mathrm{~mm}$.

HEaD. Head width (dorsal view) 1.4-1.5 times its median length, 1.1-1.2 times width of mesoscutum. Head behind eyes (dorsal view) weakly and convex-roundly narrowed. Transverse diameter of eye 1.5-1.7 times longer than temple. Ocelli arranged in triangle with base 1.15-1.20 times its side; POL 1.3-1.5 times Od, 0.5-0.6 times OOL. Eye large, glabrous, 1.2-1.3 times as high as broad, almost not concave opposite antennal socket. Malar space $0.35-0.40$ times eye height, $0.7-0.9$ times basal width of mandible. Face width 0.9-1.0 times eye height and 1.3-1.4 times height of face and clypeus combined. Malar suture absent. Clypeus rather narrow and high. Hypoclypeal depression oval, its width almost equal to distance from edge of depression to eye, $0.40-0.45$ times width of face. Occipital carina joined with hypostomal carina by additional subtransverse carina on short distance upper base of mandible. Hypostomal flange narrow.

AnTENNA. Antenna weakly thickened, filiform, 20-23-segmented, 0.6-0.7 times as long as body. Scape weakly elongate, 1.7-1.8 times longer than maximum width, 1.8-2.0 times longer than pedicel. First flagellar segment 4.5-5.0 times longer than its apical width, 1.0-1.1 times as long as second segment. Penultimate segment 1.8-2.2 times longer than its width, about 0.5 times as long as first segment, $0.7-$ 0.9 times as long as apical segment; latter acuminate apically and usually with short 'spine'.

Mesosoma. Mesosoma not depressed dorsoventrally, it length 2.0-2.1 times its height. Neck of prothorax weakly elongate, with weak and undulate pronotal carina in anterior 0.3. Mesoscutum (lateral view) not high and curvedly elevated above pronotum; its width (dorsal view) almost equal to median length. Notauli wide, shallow, complete, rather distinct posteriorly, densely rugose. Median lobe of mesoscutum (dorsal view) distinctly convex anteriorly, without median furrow. Prescutellar depression (scutal sulcus) rather deep, with three-five carinae, finely rugulose to smooth between carinae, $0.25-0.35$ times as long as scutellum. Scutellum convex, with carinae laterally, 1.1-1.2 times wider anteriorly than its median length. Subalar depression rather deep, narrow, rugose-reticulate. Precoxal sulcus mainly shallow but weakly deeper posteriorly, straight, reticulate-rugulose, running about along anterior 0.5 of lower part of mesopleuron. Metapleural flange wide and short, rounded distally. Propodeum without lateral tubercles.

WiNGS. Length of fore wing 2.8-3.0 times its width; wing almost glabrous or with very short setae in basal third, its distal margin mainly glabrous. Radial (marginal) cell strongly narrowed, 2.9-3.0 times longer than maximum width. Metacarpus (1-R1) $0.30-0.35$ times as long as pterostigma, $0.3-0.4$ times as long as distance from apex of radial (marginal) cell to apex of wing. Radial vein (r) arising before middle of pterostigma, from its basal 0.4 . First radial abscissa (r) $0.25-0.30$ times as long as distinctly and evenly curved second abscissa (3-SR + SR1), 0.6-0.8 times as long as first radiomedial vein (2-SR). Second radiomedial vein $(\mathrm{r}-\mathrm{m})$ always absent. First medial abscissa $(1-\mathrm{SR}+\mathrm{M})$ distinctly curved. Recurrent vein $(\mathrm{m}-\mathrm{cu})$ weakly antefurcal, 2.5-5.0 times longer than second abscissa of medial vein (2-SR+M). Distance from nervulus (cu-a) to basal vein (1-M) 0.5-0.8 times nervulus (cu-a) length. Hind wing 4.1-4.5 times longer than wide. First abscissa of costal vein $(\mathrm{C}+\mathrm{SC}+\mathrm{R})$ 0.7-0.9 times as long as second abscissa (1$\mathrm{SC}+\mathrm{R})$. Basal vein (1r-m) 0.7-1.0 times as long as third costal abscissa (2-SC+R). Medial (basal) cell rather narrow, weakly widened posteriorly, 7.0-8.0 times longer than wide. First abscissa of mediocubital vein $(\mathrm{M}+\mathrm{CU})$ 1.3-1.6 times longer than second abscissa (1-M). Recurrent vein (m-cu) straight or weakly curved, oblique towards base of wing, interstitial or weakly antefurcal, unsclerotised.

Legs. Hind femur 3.1-3.2 times longer than wide. Hind tibia distinctly thickened. Hind tarsus 1.1-1.2 times longer than hind tibia. Hind basitarsus weakly thickened, 0.6-0.7 times as long as second-fifth segments combined. Second segment of hind tarsus $0.55-0.60$ times as long as basitarsus, $1.5-1.7$ times longer than fifth segment (without pretarsus). 
Metasoma. Metasoma 1.1-1.2 times longer than mesosoma and head combined. First metasomal tergite rather short and wide, with weak spiracular protuberances in anterior third, distinctly and weakly convex widened from base to subapex, then narrowed towards apex. Length of first tergite 0.9-1.1 times its maximum subposterior width; its posterior width 0.9 times its subposterior width, 2.1-2.2 times its minimum anterior width. Second tergite without oblique sublateral depressions. Median length of second tergite $0.45-0.50$ times its anterior width, 1.3-1.5 times length of third tergite. Second suture distinct but fine, weakly sinuate and with weak, shallow sublateral breaks. Third tergite with very shallow and very weak transverse submedial furrow. Ovipositor sheath 1.0-1.1 times as long as metasoma, 1.5-1.6 times as long as mesosoma, $0.85-0.90$ times as long as fore wing.

SCULPTURE AND PUBESCENCE. Vertex densely transversely undulately striate with dense reticulation; frons entirely densely rugose-reticulate; face densely and curvedly transversely striate with rugulosity, rugose with as few striae upper medially and lower laterally; temple mainly reticulate-rugulose, finely granulate to coriaceous in narrow subvertical stripe near eyes. Mesoscutum densely distinctly granulate, rugulose and undulately striate in rather wide area in medioposterior half. Scutellum finely coriaceous, almost smooth medially. Mesopleuron mostly densely reticulation, finer below. Metapleuron entirely densely rugose-reticulate. Propodeum without areas delineate by carinae, entirely and densely rugosereticulate. Hind coxa mainly densely reticulate-coriaceous, dorsally with additional rugosity in base, finely sculptured in lower half. Hind femur distinctly reticulate in dorsal half, finely reticulate to smooth in lower half. First metasomal tergite entirely distinctly striate, with dense rugulosity between striae, without distinct dorsal carinae. Second tergite entirely densely striate and with dense rugulosity between striae. Third tergite narrowly medially and wide laterally densely reticulate, finely coriaceous posteriorly, smooth in posterior three-fourths (medially) to one-fourths (laterally). Fourth to sixth tergites densely and finely to very finely reticulate-coriaceous in anterior two-thirds to half, smooth on remaining distal parts. Vertex with sparse, short and semi-erect yellowish setae. Mesoscutum widely glabrous, with sparse, short and semi-erect white setae arranged narrow along notauli and laterally and widely on median area. Mesopleuron medially widely glabrous. Hind tibia dorsally with short, sparse and semierect pale setae, their length $0.2-0.3$ times maximum width of tibia.

Colour. Body reddish brown, partly with light or dark reddish brown spots; metasoma laterally sometimes brownish yellow. Antenna light brown to light reddish brown in basal two- or three-fourths, brown to dark brown apically. Palpi yellow. Fore leg ma yellowish brown, middle and hind legs reddish brown; trochanter, trochantellus, base of tibia at short distance and almost all tarsi pale yellow. Ovipositor sheath brownish yellow to light brown in anterior half, brown to almost black in posterior half. Fore wing maculate, partly hyaline with distinct wide transverse stripes or areas around basal vein and on and below radial (marginal) cell. Pterostigma dark brown, pale yellow in basal quarter and yellow anteriorly.

\author{
Male \\ Unknown.
}

\title{
Distribution
}

UAE.

Hecabalodes radialis Tobias, 1962

Figs 30-31

Hecabalodes radialis Tobias, 1962: 275.

Hecabalodes radialis - Yu et al. 2016. 

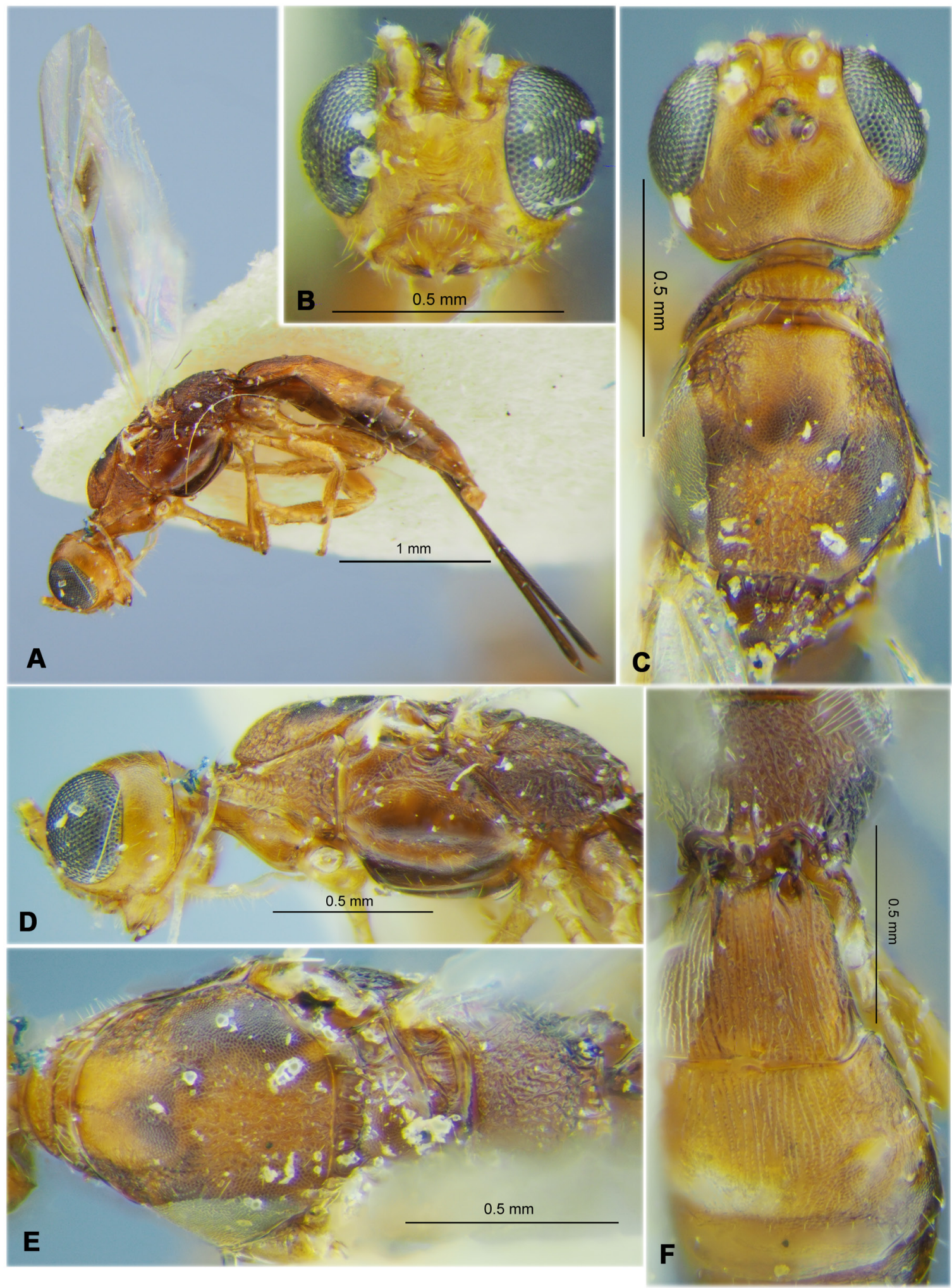

Fig. 30. Hecabalodes radialis Tobias, 1962, paratype, $q$ (ZISP). A. Habitus, lateral view. B. Head, front view. C. Head and mesoscutum, dorsal view. D. Head and mesosoma, lateral view. E. Mesosoma, dorsal view. F. Propodeum and first to third metasomal tergites, dorsal view. 

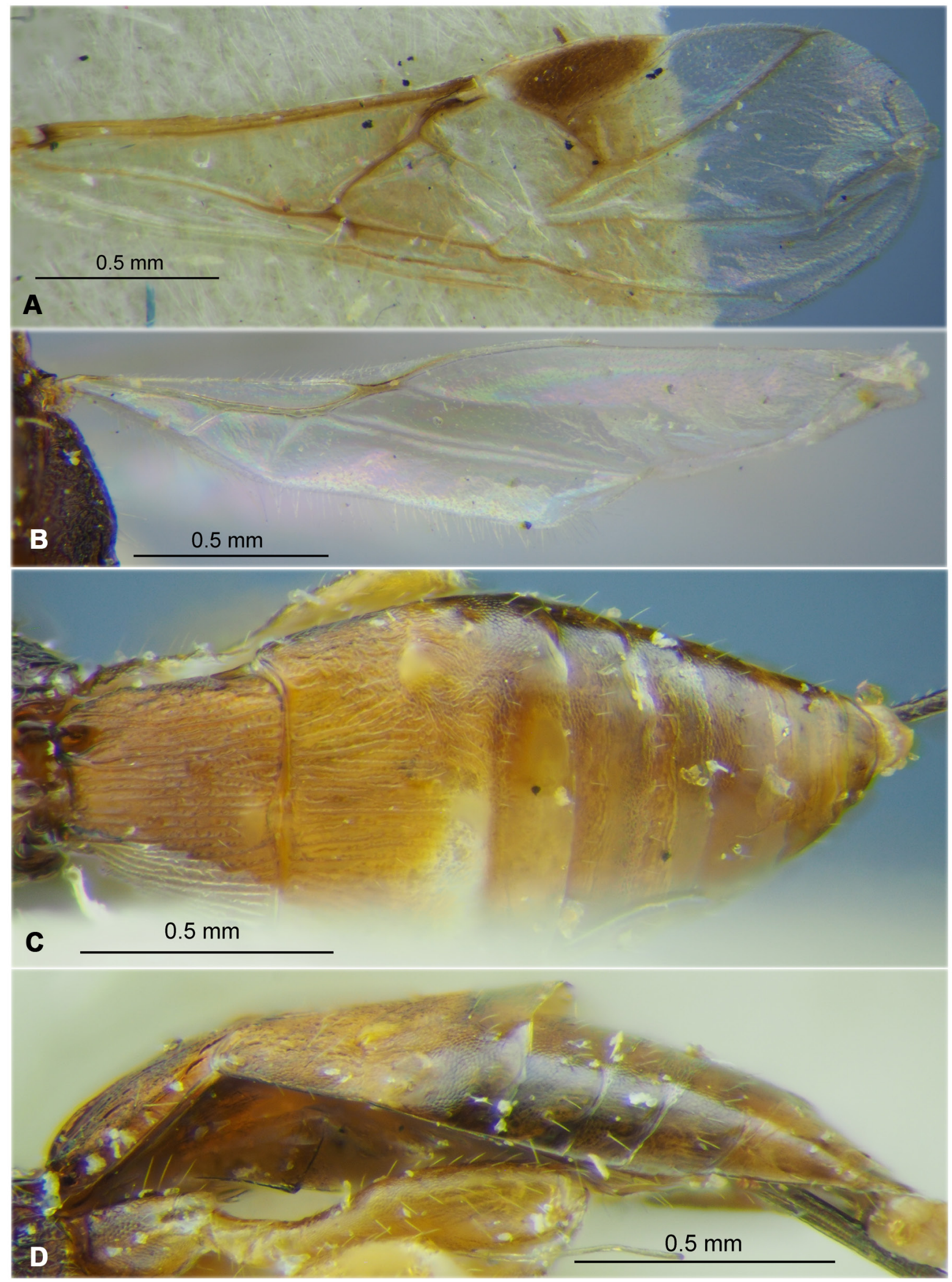

Fig. 31. Hecabalodes radialis Tobias, 1962, paratype, $q$ (ZISP). A. Fore wings. B. Hind wing. C. Metasoma, dorsal view. D. Metasoma, lateral view. 


\section{Material examined}

\section{Holotype}

TURKMENISTAN • 1 (head and antennae missing); "Turkmenia, Badkhyz Nature Reserve, ex Acmaeodera sp., 9.viii.1955, Znamenskiy coll.”; ZISP.

\section{Addititonal material}

UNITED ARAB EMIRATES • 2 9 ; “ “United Arab Emirates, Sharjah (96), light trap, 11-17.xi.2004, $25^{\circ} 21^{\prime}$ N, 55 24' E, A.v. Harten, RNMH'05"; RMNH • 1 q; "United Arab Emirates, Sharhah x Khor Kalba (6327-6337), light trap, 24-30.vi.2006, A.v. Harten, RMNH'06”; RMNH • 6 우; “United Arab Emirates, SSW of ad-Dhaid (6154), light tr., 24-30.v.2006, $25^{\circ} 09^{\prime} \mathrm{N}, 55^{\circ} 48^{\prime}$ E, A.v. Harten, RMNH'06"; RMNH • 2 $9+$; same label data as for preceding; ZISP • 1 q; "United Arab Emirates, SSW

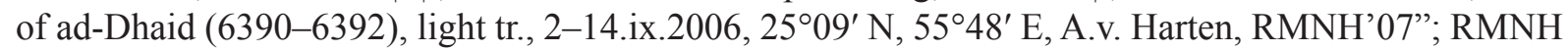
- 1 क; 'United Arab Emirates, Wadi Maidaq (6474), light trap, 27.iv-4.v.2006, $25^{\circ} 18^{\prime}$ N, $56^{\circ} 07^{\prime}$ E, A.v. Harten, RNMH'07”; RMNH - + ; “United Arab Emirates, Wadi Maidaq (6452-6456), water trap, 24.ix-22.x.2006, $25^{\circ} 18^{\prime}$ N, 56 07' E, A.v. Harten, RNMH'07”; RMNH • 1 O; "United Arab Emirates,

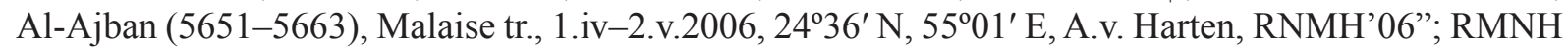

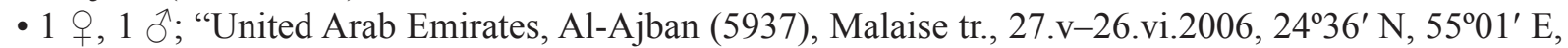
A.v. Harten, RNMH'06"; RMNH • 1 đ’; "United Arab Emirates, Al-Ajban (6528), Malaise \& light tr.,

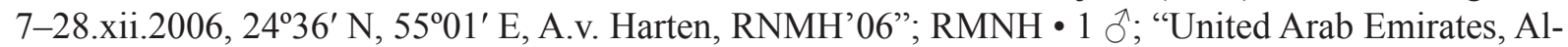

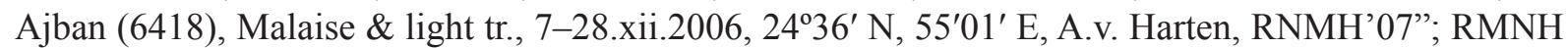

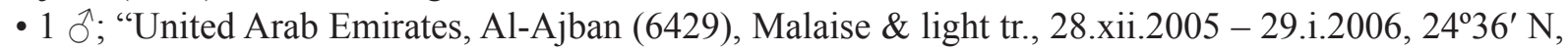
5501' E, A.v. Harten, RNMH'07”; RMNH • 1 ㅇ; “United Arab Emirates, Wadi Safad (5867-5902), at light, 31.i-21.ii.2006, $25^{\circ} 13^{\prime} \mathrm{N}, 56^{\circ} 19^{\prime}$ E, A.v. Harten, RNMH'06”; RMNH • 2 우; “United Arab Emirates, Wadi Safad (5020), at light, 14-21.v.2006, $25^{\circ} 13^{\prime}$ N, 56 ${ }^{\circ} 19^{\prime}$ E, A.v. Harten, RNMH'06”; RMNH

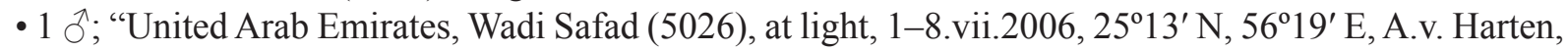
RNMH'06"; RMNH • 1 đ'; same label data as for preceding; ZISP • 5 o $\circ$; "United Arab Emirates, Wadi

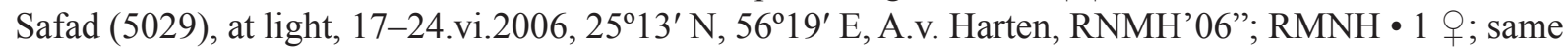
label data as for preceding; ZISP • 1 + 1 đ; "United Arab Emirates, Wadi Safad (5550), at light, 1-8. vii.2006, $25^{\circ} 1^{\prime}$ N, $56^{\circ} 1^{\prime}$ E, A.v. Harten, RNMH'06"; RMNH • 3 ㅇ; “"United Arab Emirates, Wadi Safad

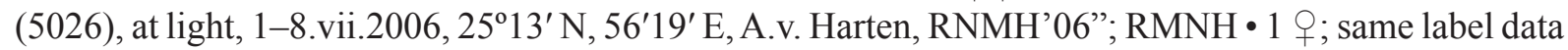

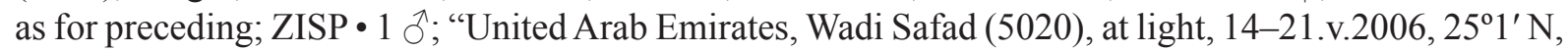
$56^{\circ} 1^{\prime}$ E, A.v. Harten, RNMH'06"; RMNH 1 ơ; "United Arab Emirates, Hatta (6398), at light, 17-24.

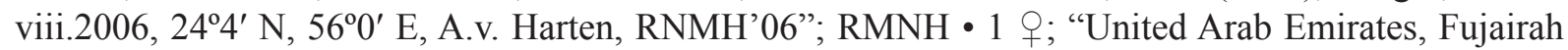

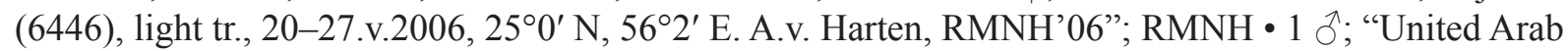

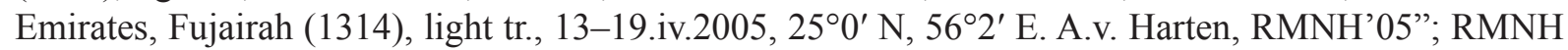

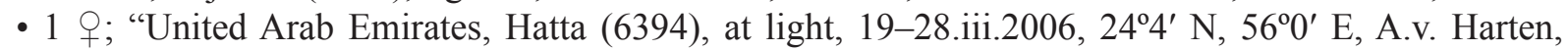
RNMH'06"; RMNH • 1 क; "United Arab Emirates, Sharjah Desert Park (1308), light tr., 6-13.iv.2005, $25^{\circ} 17^{\prime}$ N, $55^{\circ} 4^{\prime}$ E, A.v. Harten, RNMH'05”; RMNH • 1 O; "United Arab Emirates, Sharjah Desert Park

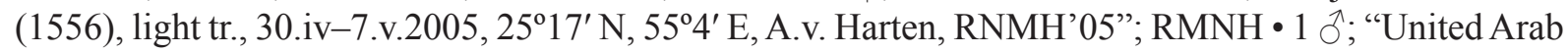

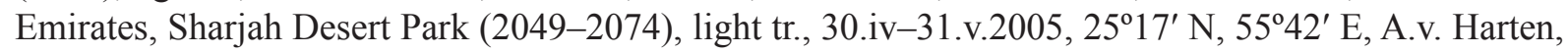
RNMH'05"; RMNH • 2 우; “United Arab Emirates, Sharjah Desert Park (5704-5740), light tr., 25.ii-

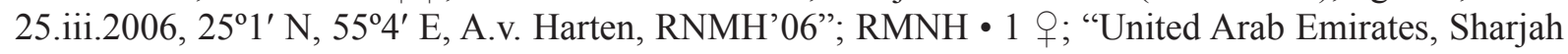

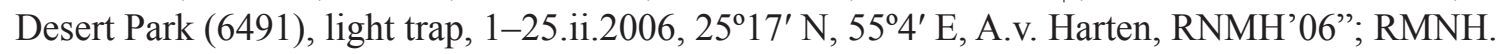

YEMEN • 1 क; "Yemen (3901), Al Kowd, vii.1999, light-trap, A.v. Harten \& S. Al Haruri. RMNH'00"; RMNH • 1 क; "Yemen (4054), Al Kowd, viii.1999, light-trap, A.v. Harten \& S. Al Haruri. RMNH'00"; RMNH • 2 ㅇ; " "Yemen (4055), Al Kowd, ix.1999, light-trap, A.v. Harten \& S. Al Haruri, RMNH'00"; RMNH • 1 \%; "Yemen, Ta'izz, viii.1999, light tr., no 4056, A.v. Harten \& A. Awad, RMNH'01”; RMNH. 
BELOKOBYLSKIJ S.A. \& VAN ACHTERBERG C., Review of Doryctinae from UAE and Yemen

\section{Distribution}

Greece, India, Iran, Tajikistan, Turkey, Turkmenistan, *UAE, *Yemen.

Hecabalodes xylophagi Fischer, 1962

Figs 32-33

Hecabalodes xylophagi Fischer, 1962: 299.

Hecabalodes xylophagi - Yu et al. 2016.

\section{Material examined}

UNITED ARAB EMIRATES • 1 q ; "United Arab Emirates, Wadi Safad (5867-5902), at light, 31.i-21.

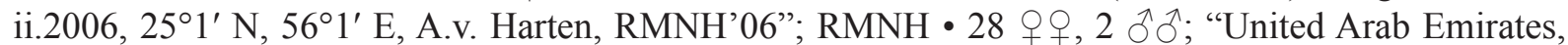

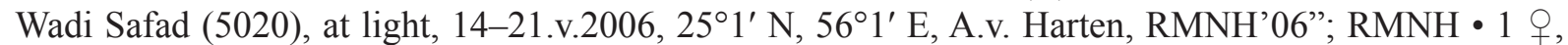
1 '; same label data as for preceding; ZISP • 1 q; "United Arab Emirates, Wadi Safad (6435), at light, 14-21.vi.2006, $25^{\circ} 1^{\prime} \mathrm{N}, 56^{\circ} 1^{\prime}$ E, A.v. Harten, RMNH'06”; RMNH • 3 o ox; "United Arab Emirates,

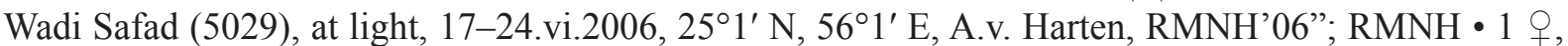

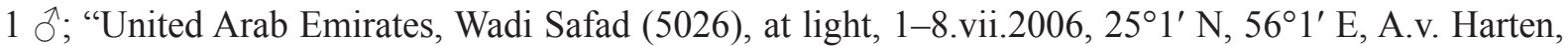

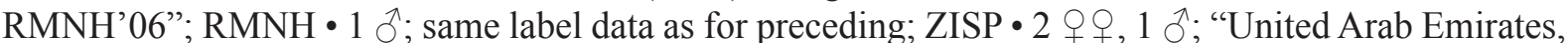
Sharhah x Khor Kalba (6599), light trap, 24-30.v.2006, A.v. Harten, RMNH'06"; RMNH • 19 오, 6 วิ수; "United Arab Emirates, Sharhah x Khor Kalba (6308-6311), light trap, 31.v-7.vi.2006,

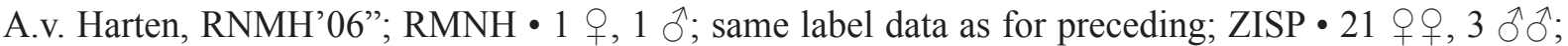
"United Arab Emirates, Sharhah x Khor Kalba (6327-6337), light trap, 24-30.vi.2006, A.v. Harten,

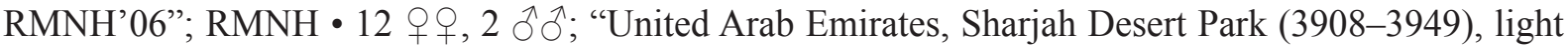
tr., 20-21.iv.2006, $25^{\circ} 17^{\prime} \mathrm{N}, 55^{\circ} 42^{\prime}$ E, M. Fibiger, RNMH'06"; RMNH • 1 \%; "United Arab Emirates,

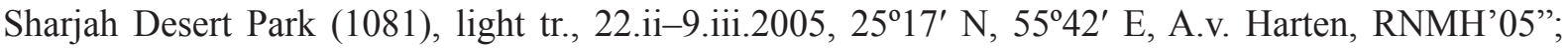

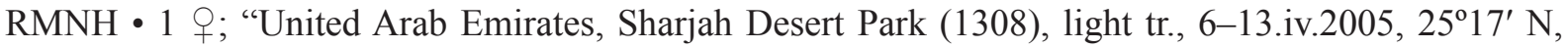
$55^{\circ} 42^{\prime}$ E, A.v. Harten, RNMH'05”; RMNH • 1 \%; "United Arab Emirates, Sharjah Desert Park (1394),

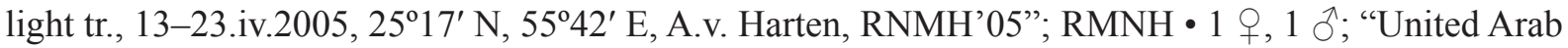

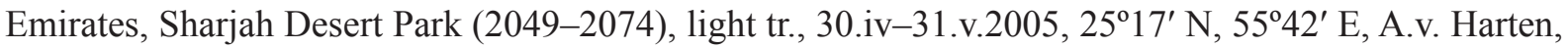
RNMH'05”; RMNH - 2 우; “United Arab Emirates, Sharjah Desert park (2517), light trap, 20.x-8. xi.2005, $25^{\circ} 17^{\prime} \mathrm{N}, 55^{\circ} 42^{\prime}$ E, A.v. Harten, RNMH'06”; RMNH • 2 우, $1 \delta^{\lambda}$; “United Arab Emirates, Sharjah

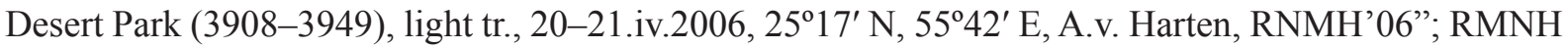
- 2 우우; “United Arab Emirates, Sharjah Desert Park (5704-5740), light tr., 25.ii-25.iii.2006, 2517' N, $55^{\circ} 42^{\prime}$ E, A.v. Harten, RNMH'06”; RMNH - 2 q 9 ; “United Arab Emirates, Sharjah (514), light trap,

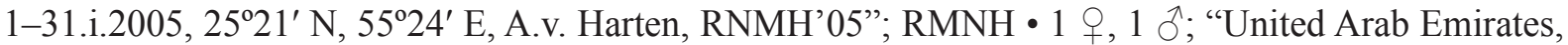

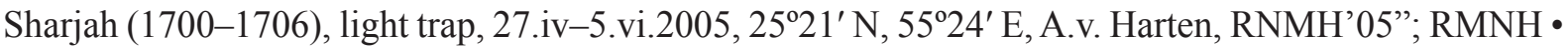

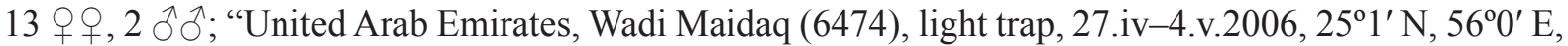
A.v. Harten, RNMH'07”; RMNH • 1 ภ; "United Arab Emirates, Al-Ajban (6429), Malaise \& light tr.,

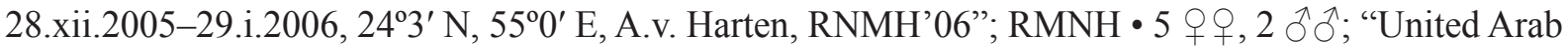

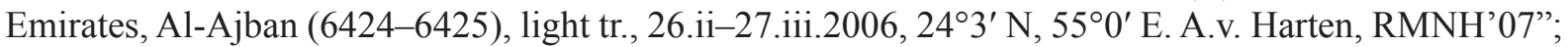

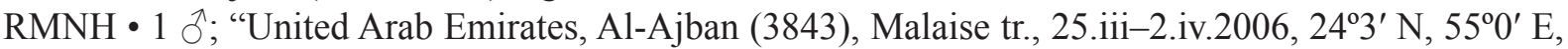
A.v. Harten, RNMH'06"; RMNH • 2 o $ᄋ$; "United Arab Emirates, Al-Ajban (6422), Malaise tr., 26.ii-2. iv.2006, $24^{\circ} 3^{\prime} \mathrm{N}, 55^{\circ} 0^{\prime}$ E, A.v. Harten, RNMH'07"; RMNH • 1 đ; "United Arab Emirates, Al-Ajban

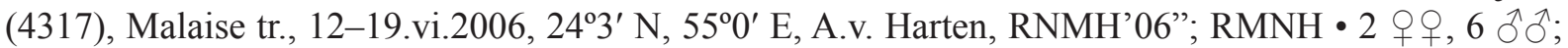

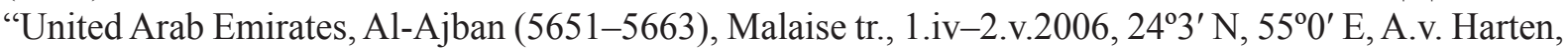
RNMH'06”; RMNH • 7 q ; “ "United Arab Emirates, Al-Ajban (5937), Malaise tr., 27.v-26.vi.2006, $24^{\circ} 36^{\prime} \mathrm{N}, 55^{\circ} 01^{\prime}$ E, A.v. Harten, RNMH'06"; RMNH • 2 우; “United Arab Emirates, Al-Ajban (6426),

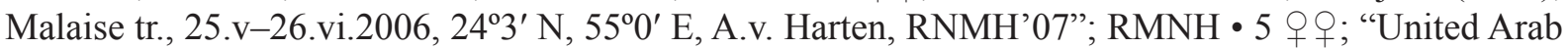
Emirates, SSW of ad-Dhaid (6154), light tr., 24-30.v.2006, $25^{\circ} 09^{\prime}$ N, 55 $45^{\prime}$ E, A.v. Harten, RNMH'06”; 


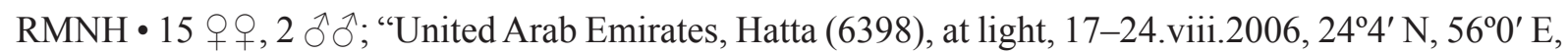

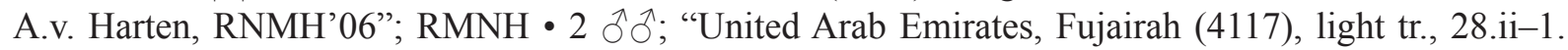

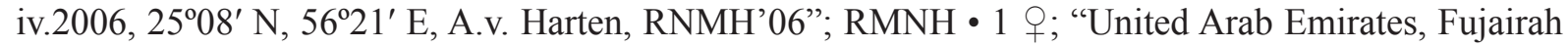
(1224), light tr., 5-24.iii.2005, 250' N, 56²' E. A.v. Harten, RMNH’06”; RMNH • 4 우; “United Arab

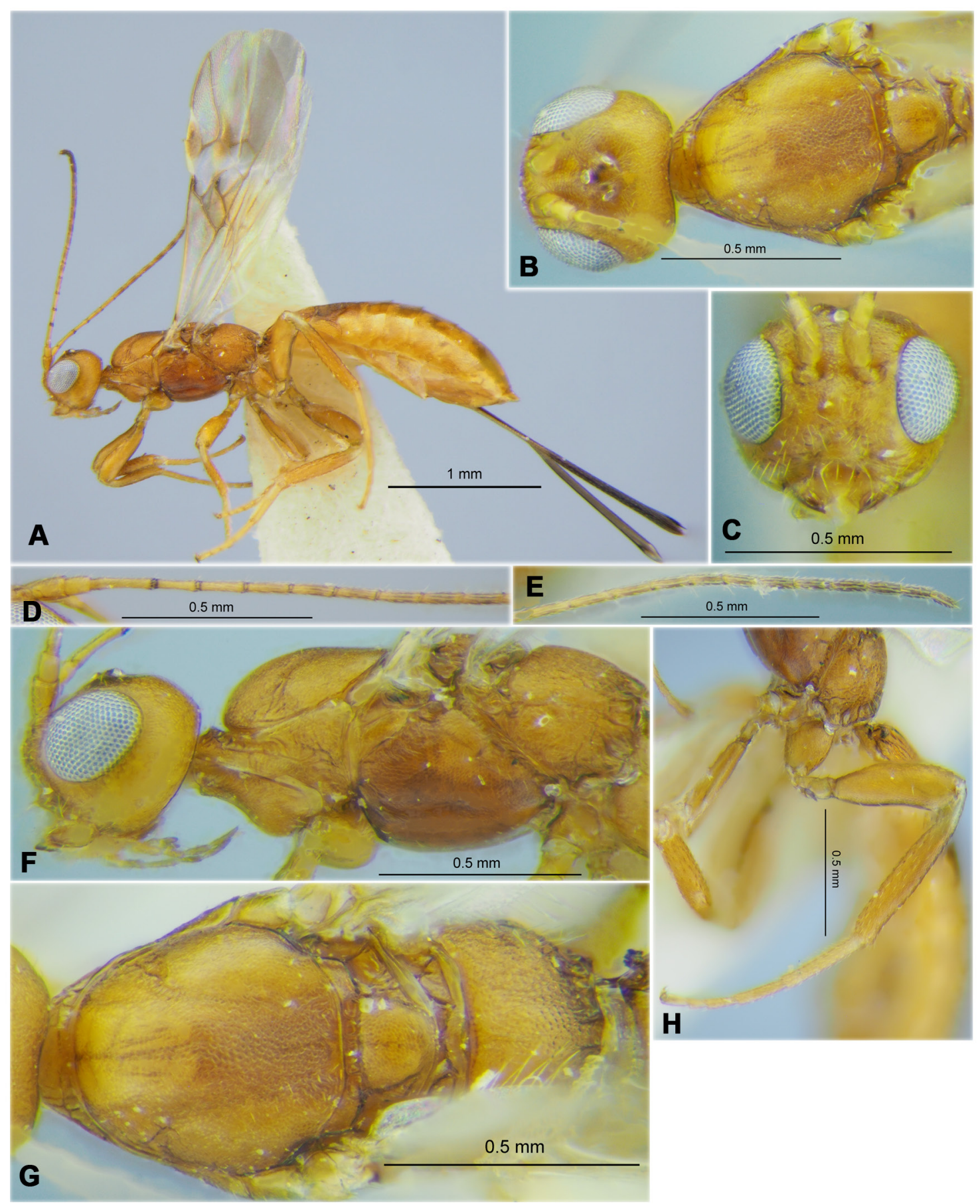

Fig. 32. Hecabalodes xylophagi Fischer, 1962, $q$ (RMNH). A. Habitus, lateral view. B. Head and mesoscutum, dorsal view. C. Head, front view. D. Basal segments of antenna. E. Apical segments of antenna. F. Head and mesosoma, lateral view. G. Mesosoma, dorsal view. H. Hind leg. 
Emirates, Fujairah (1314), light tr., 13-19.iv.2005, 250' N, 56²' E. A.v. Harten, RMNH'05”; RMNH

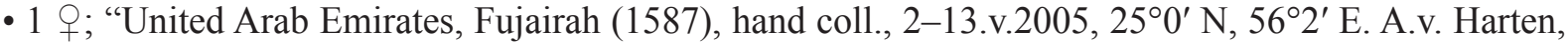
RMNH'05”; RMNH • 3 우우 “ “United Arab Emirates, Fujairah (6446), light tr., 20-27.v.2006, 250' N, $56^{\circ} 2^{\prime}$ E. A.v. Harten, RMNH'06”; RMNH - 1 o; "United Arab Emirates, Bithnah (3699), at light,

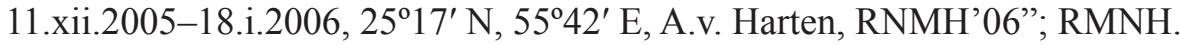
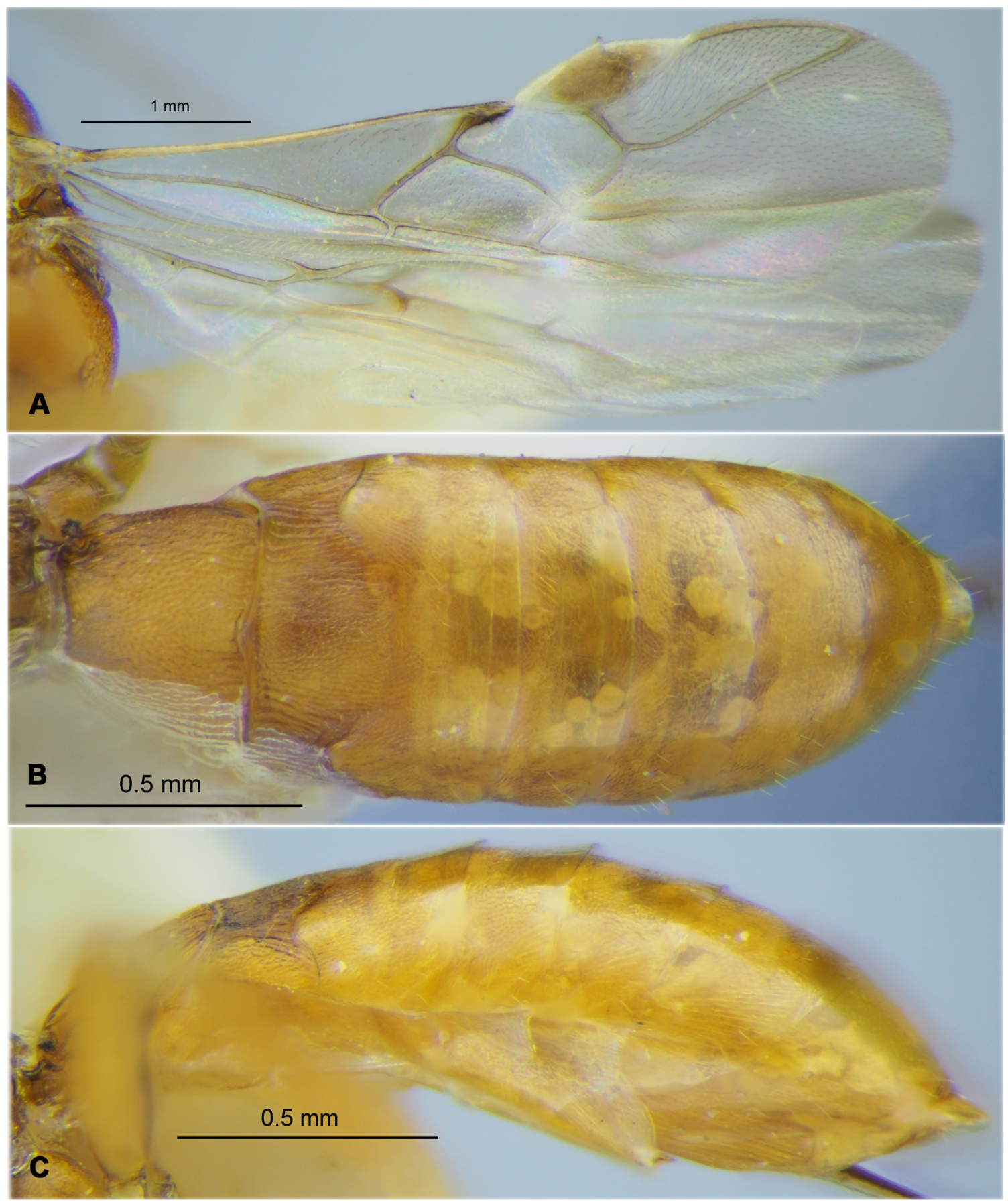

Fig. 33. Hecabalodes xylophagi Fischer, 1962, ㅇ (RMNH). A. Wings. B. Metasoma, dorsal view. C. Metasoma, lateral view. 
YEMEN • 3 o o ; “Yemen (3901), Al Kowd, vii.1999, light-trap, A.v. Harten \& S. Al Haruri. RMNH'00”; RMNH • 3 우, 1 đ̃; "Yemen (4054), Al Kowd, viii.1999, light-trap, A.v. Harten \& S. Al Haruri, RMNH'00"; RMNH; 1 q; same label data as for preceding; ZISP • 1 +; "Yemen (4055), Al Kowd, ix.1999, light-trap, A.v. Harten \& S. Al Haruri, RMNH'00"; RMNH・15 우, 2 ふぇં; "Yemen, Al Kowd, v-vi.2000, light-tr., no 4719, A.v. Harten \& S. Al Haruri, RMNH'01”; RMNH; 2 ㅇ $\circ$; same label data as for preceding; ZISP • 2 क $\circ$; "Yemen, Al Kowd, vii.2000, light tr., no 4820, A.v. Harten \& S. Al Haruri, RMNH'01"; RMNH • 1 q; "Yemen, Al Kowd, ii.2001, light tr., no. 5484, A.v. Harten \& S. Al Haruri, RMNH'01"; RMNH • 1 +; "Yemen (5750), Al Kowd, 8-12.vii.2001, light trap, A.v. Harten \& S. Al Haruri. RMNH'02"; RMNH • 2 우; "Yemen (6141), Al Kowd, 16-20.viii.2001, light trap, A.v. Harten \& S. Al Haruri. RMNH'02"; RMNH • 5 웅, 1 ㄱ; "Yemen (8136), Al Kowd, ix.2003, light trap, A.v. Harten, S. Al Haruri, RMNH'03"; RMNH • 1 q; "Yemen (7189), Al Kadan, v.2002, Light trap, A.v. Harten \& A.R. Al Yarimi. RMNH'02"; RMNH • 2 o $\circ$; "Yemen (5404), Hammam 'Ali, from coffee-berries (with Ceratitis capitata ?), 14.iii.2001, A.v. Harten, RMNH'02"; RMNH.

\section{Distribution}

Algeria, Chad, Mauritania, *UAE, *Yemen.

Genus Leluthia Cameron, 1887

\section{Type species}

Leluthia mexicana Cameron, 1887, designated by Viereck (1914).

\section{Remarks}

This widely distributed genus consist of two subgenera, Euhecabolodes Tobias, 1962 and Leluthia s. str. Species of Leluthia were recently recorded in the fauna of Afrotropical region for the first time by Belokobylskij (2020b).

\section{Distribution}

Afrotropical, Australasian, Nearctic, Neotropical and Palaearctic regions.

\section{Key to Arabian species of the genus Leluthia}

1. Notauli on dorsal part of mesoscutum subparallel. Second suture strongly evenly curved forwards and without sublateral breaks. Second metasomal tergite without sublateral furrows. Pterostigma pale brown, yellow basally and apically. Body length 1.6-3.1 mm. - Yemen

Leluthia (L.) abnormis Belokobylskij, 2020

- Notauli on dorsal part of mesoscutum convergent posteriorly. Second suture medially curved posterior and with distinct sublateral breaks. Second metasomal tergite strongly oblique sublateral furrows. Pterostigma mainly yellow. Body length 1.7-3.3 mm. - Yemen

Leluthia (L.) brevitergum Belokobylskij, 2020

Leluthia (Leluthia) abnormis Belokobylskij, 2020

Leluthia abnormis Belokobylskij, 2020b: 285.

\section{Material examined}

Holotype

YEMEN • १; "Yemen (4055), Al Kowd, ix.1999, light-trap, A.v. Harten \& S. Al Haruri, RMNH'00”; RMNH. 


\section{Paratypes}

YEMEN • 3 우, 4 ठึ đึ; "Yemen (4054), Al Kowd, viii.1999, light-trap, A.v. Harten \& S. Al Haruri,

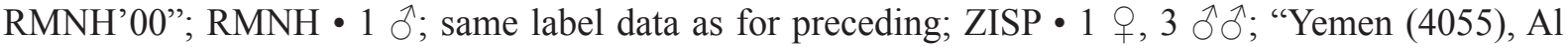
Kowd, ix.1999, light-trap, A.v. Harten \& S. Al Haruri, RMNH'00"; RMNH • 1 'े; same label data as for preceding; ZISP • 3 웅, 1 ㄱ; "Yemen (3901), Al Kowd, vii.1999, light-trap, A.v. Harten \& S. Al Haruri. RMNH'00"; RMNH・ 1 क; "Yemen (6141), Al Kowd, 16-20.viii.2001, light trap, A.v. Harten \& S. Al Haruri. RMNH'02"; RMNH • 1 क; "Yemen (8136), Al Kowd, ix.2003, light trap, A.v. Harten, S. Al Haruri, RMNH'03"; RMNH • 6 우, 1 ○’; "Yemen (5404), Hammam 'Ali, from coffee-berries (with Ceratitis capitata ?), 14.iii.2001, A.v. Harten, RMNH'02"; RMNH • 1 क; same label data as for preceding; ZISP • 4 우; "Yemen, Al Kowd, v-vi.2000, light-tr., no 4719, A.v. Harten \& S. Al Haruri, RMNH'01"; RMNH • 1 क; same label data as for preceding; ZISP • 1 q; "Yemen, Al Kowd, vii.2000, light tr., no 4820, A.v. Harten \& S. Al Haruri, RMNH'01"; RMNH • 1 o; "Yemen (5750), Al Kowd, 8-12.vii.2001, light trap, A.v. Harten \& S. Al Haruri. RMNH'02"; RMNH • 2 §ิ ô; "Yemen (8136), Al Kowd, ix.2003, light trap; A.v. Harten \& S. Al Haruri. RMNH'02"; RMNH • 1 §े; "Yemen, Al Kowd, viii.2000, light-tr., no 4952, A.v. Harten \& S. Al Haruri, RMNH'01"; RMNH • 2 §ో ठૈ; "Yemen, Al Kowd, v-vi.2000, light-tr., no 4719, A.v. Harten \& S. Al Haruri, RMNH'01”; RMNH

\section{Additional material}

YEMEN • 1 đ̊; "Yemen, Ta'izz, viii.1999, light tr., A.v. Harten \& A Awad, RMNH’01”; RMNH.

\section{Distribution}

Yemen.

Leluthia (Leluthia) brevitergum Belokobylskij, 2020

Leluthia brevitergum Belokobylskij, 2020b: 290.

\section{Material examined}

\section{Holotype}

YEMEN • O; "Yemen (3901), Al Kowd, vii.1999, light-trap, A.v. Harten \& S. Al Haruri. RMNH'00"; RMNH.

\section{Paratypes}

YEMEN • 1 ठ̊?; "Yemen (3901), Al Kowd, vii.1999, light-trap, A.v. Harten \& S. Al Haruri. RMNH'00"; RMNH • 1 đ;; same label data as for preceding; ZISP • 1 \&; "Yemen (4054), Al Kowd, viii.1999, lighttrap, A.v. Harten \& S. Al Haruri, RMNH'00"; RMNH • 1 q; same label data as for preceding; ZISP • 1 गे; "Yemen, Al Kowd, ii.2000, light tr., no 4431, A.v. Harten \& S. Al Haruri. RMNH'01"; RMNH • 2 우, 1 ð’; "Yemen, Al Kowd, v-vi.2000, light tr., no. 4719, A.v. Harten \& S. Al Haruri. RMNH'01"; RMNH • 1 Oे; "Yemen (8136), Al Kowd, ix.2003, light trap, A.v. Harten, S. Al Haruri, RMNH'03"; RMNH • 1 +, 1 §’; "Yemen (5404), Hammam"Ali, from coffee-berries (with Ceratitis capitata ?), 14.iii.2001, A.v. Harten, RMNH'02"; RMNH.

\section{Distribution}

Yemen.

Genus Parallorhogas Marsh, 1993

\section{Type species}

Allorhogas pyralophagus Marsh, 1984, by original designation. 


\section{Remarks}

This is a relatively small genus with most species recorded from the Oriental and Eastern Palaearctic regions (Belokobylskij \& Maetô 2009).

\section{Distribution}

Afrotropical, Oceanic, Oriental, Nearctic, Neotropical and Palaearctic regions,

Parallorhogas testaceus (Szépligeti, 1914) comb. nov.

Figs 34-35

Coeloreuteus testaceus Szépligeti, 1914: 203

Coeloreuteus testaceus - Shenefelt 1975: 1193.

Opius (Opius) testaceus - Yu et al. 2016.

\section{Comparative diagnosis}

This species is similar to the Indian P. colophon (Nixon, 1939), but differs from the latter species by having the body small (large in P. colophon), antenna 17-18-segmented (29-segmented in P. colophon), first flagellar segment short, 3.5-4.0 times longer than its apical width and as long as second segment (long, 4.5 and 1.3 times accordingly in $P$. colophon), recurrent vein (m-cu) of fore wing postfurcal or rarely subinterstitial (antefurcal in $P$. colophon), scutellum finely but distinctly reticulate-coriaceous (smooth in P. colophon) and mesoscutum almost entirely setose (setose only along notauli and laterally in P. colophon).

Parallorhogas testaceus is also similar to the Malagasy Monolexis caudatus Szépligeti, 1913, but differs from it in having the second radiomedial vein $(\mathrm{r}-\mathrm{m})$ developed (always absent in the genus Monolexis, including M. caudatus), transverse diameter of eye 1.6-1.8 times longer than temple (2.3 times in $M$. caudatus), radial vein of fore wing (r) arising distinctly before middle of pterostigma (from middle in M. caudatus), nervellus (m-cu) of hind wing present (absent in M. caudatus), length of first tergite almost equal to its posterior width (1.2 times larger in M. caudatus) and ovipositor shorter than metasoma (distinctly longer in M. caudatus).

\section{Material examined}

Holotype

TOGO • +; “Togo, Kete Kratje, Graf Zech S., vii.1899”, “Coeloreutes testaceum (sic!) Sz.” (handwriting by Szépligeti); MNBG.

\section{Additional material}

UNITED ARAB EMIRATES • 1 q; "United Arab Emirates, Sharjah (514), light trap, 1-31.i.2005,

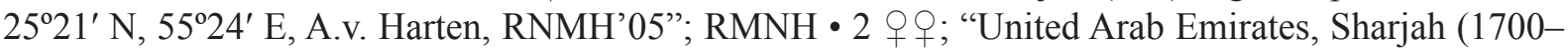

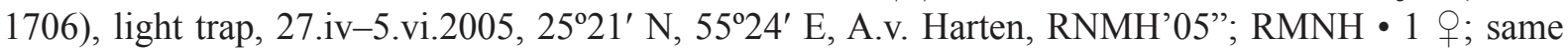
label data as for preceding; ZISP.

\section{Redescription}

\section{Female}

Measurements. Body length 1.9-2.8 mm; fore wing length 1.5-2.1 mm.

HEAD. Head width (dorsal view) 1.6-1.8 times its median length, 1.1-1.2 times width of mesoscutum. Head behind eyes (dorsal view) weakly convex in anterior half, distinctly roundly narrowed in posterior half. Transverse diameter of eye 1.6-2.0 times longer than temple. Ocelli arranged in almost equilateral 
triangle; POL 1.2-1.4 times Od, 0.3-0.4 times OOL. Eye almost glabrous, 1.20-1.25 times as high as broad. Malar space 0.3-0.4 times eye height, 0.7-0.9 times basal width of mandible. Face width 0.85-0.90 times eye height and almost equal to height of face and clypeus combined. Malar suture absent. Hypoclypeal depression subround, its width 0.6-0.8 times distance from edge of depression to eye, $0.3-0.4$ times width of face. Occipital carina not joined with hypostomal carina being obliterated ventrally on short distance upper base of mandible. Hypostomal flange very narrow.

ANTENNA. Antenna rather thick, filiform, 17-18-segmented, almost as long as body. Scape 1.3-1.5 times longer than maximum width, 1.7-2.0 times longer than pedicel. First flagellar segment 3.5-4.0 times longer than its apical width, about as long as second segment. Penultimate segment 2.7-3.0 times longer than wide, 0.8-0.9 times as long as first flagellar segment, 0.9 times as long as apical segment; latter weakly acuminated apically.

Mesosoma. Length 1.5-1.6 times its height. Neck of prothorax short, with distinct pronotal keel in anterior 0.3-0.4. Mesoscutum (lateral view) highly and perpendicularly elevated above pronotum; rather short (dorsal view), its width 1.30-1.35 times median length. Median lobe of mesoscutum (dorsal view) almost straight anteriorly, without median furrow. Notauli distinct, complete, rather narrow, sparsely distinctly crenulate. Prescutellar depression (scutal sulcus) rather deep, finely rugulose, with three-five distinct carinae, 0.35-0.40 times as long as scutellum. Subalar depression shallow, rather narrow, striate with rugosity partly. Precoxal sulcus deep, oblique, finely rugulose-crenulate to almost smooth, running along anterior half of lower part of mesopleuron. Metapleural flange narrow, subrounded distally. Propodeum without lateral tubercles.

WINGS. Length of fore wing 2.8-3.0 times its width. Metacarpus (1-R1) 1.1-1.2 times longer than pterostigma. Radial vein (r) arising before middle of pterostigma, from basal 0.35 . Second radial abscissa (3-SR) 1.2-1.6 times longer than first abscissa (r), 0.25-0.30 times as long as straight third abscissa (SR1), 0.6-0.8 times as long as first radiomedial vein (2-SR). Second radiomedial vein (r-m) present. Second radiomedial (submarginal) cell rather short, 2.3-2.7 times longer than its maximum width, 1.01.1 times as long as narrow brachial (subdiscal) cell. First medial abscissa (1-SR+M) distinctly curved and weakly sinuate. Recurrent vein (m-cu) postfurcal or sometimes subinterstitial, 3.0-4.0 times longer than second abscissa of medial vein $(2-\mathrm{SR}+\mathrm{M})$. Distance from nervulus (cu-a) to basal vein (1-M) 0.5-0.7 times nervulus length. Hind wing 4.5-5.0 times longer than wide. First abscissa of costal vein $(\mathrm{C}+\mathrm{SC}+\mathrm{R})$ 0.9-1.1 times as long as second abscissa (1-SC+R). Basal vein (1r-m) almost 1.0-1.4 times as long as third costal abscissa (2-SC+R). Medial (basal) cell narrow, weakly widened posteriorly, 7.59.0 times longer than wide, 0.3 times as long as hind wing. First abscissa of mediocubital vein $(\mathrm{M}+\mathrm{CU})$ $0.9-1.0$ times as long as second and third abscissae combined (1-M). Recurrent vein (m-cu) distinctly and evenly curved towards apex of wing, antefurcal, unsclerotised.

Legs. Hind femur 3.1-3.5 times longer than wide. Hind tibia weakly thickened. Hind tarsus almost as long as hind tibia. Hind basitarsus weakly thickened, $0.50-0.55$ times as long as second-fifth segments combined. Second segment of hind tarsus 0.6 times as long as basitarsus, 1.4-1.6 times longer than fifth segment (without pretarsus).

Metasoma. Metasoma 1.3-1.5 times longer than mesosoma and head combined. First metasomal tergite with small or very small spiracular protuberances in anterior 0.3 , distinctly and almost linearly widened from base to apex. Length of first tergite almost equal to its posterior width; posterior width 2.0-2.5 times its minimum width. Second tergite with very shallow oblique sublateral depressions. Median length of second tergite $0.35-0.40$ times its anterior width, 0.75-0.90 times length of third tergite. Second suture shallow and weakly sinuate. Third tergite without transverse furrow. Ovipositor sheath $0.8-0.9$ times as long as metasoma, 1.4-1.8 times longer than mesosoma, 0.6-0.8 times as long as fore wing. 


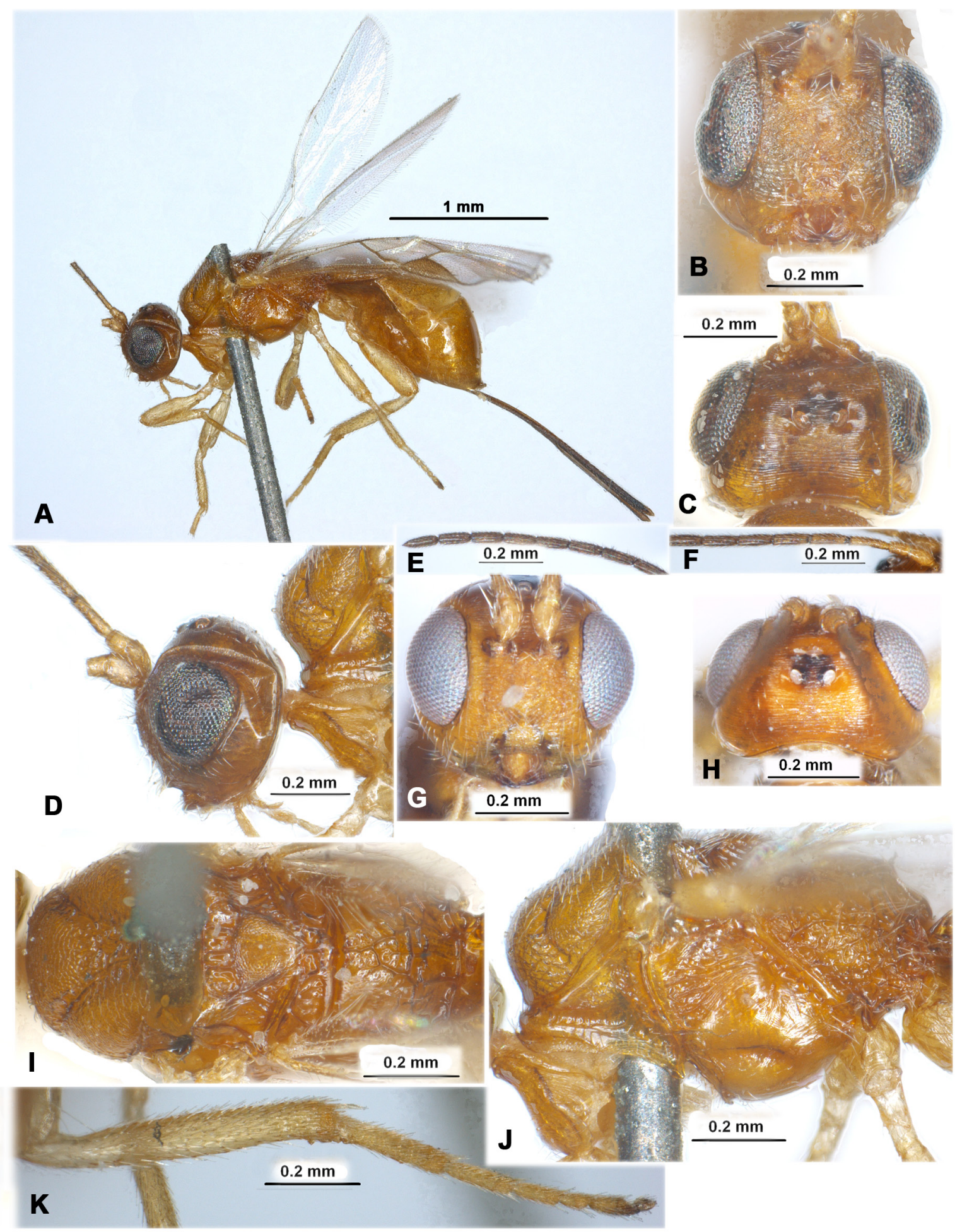

Fig. 34. Parallorhogas testaceus (Szépligeti, 1914). A-F, I-K. Holotype, + (MNBG). G-H. Arabian specimen (RMNH). A. Habitus, lateral view. B, G. Head, front view. C, H. Head, dorsal view. D. Head, lateral view. E. Apical segments of antenna. F. Basal segments of antenna. I. Mesosoma, dorsal view. J. Mesosoma, lateral view. K. Hind tibia and tarsus. 

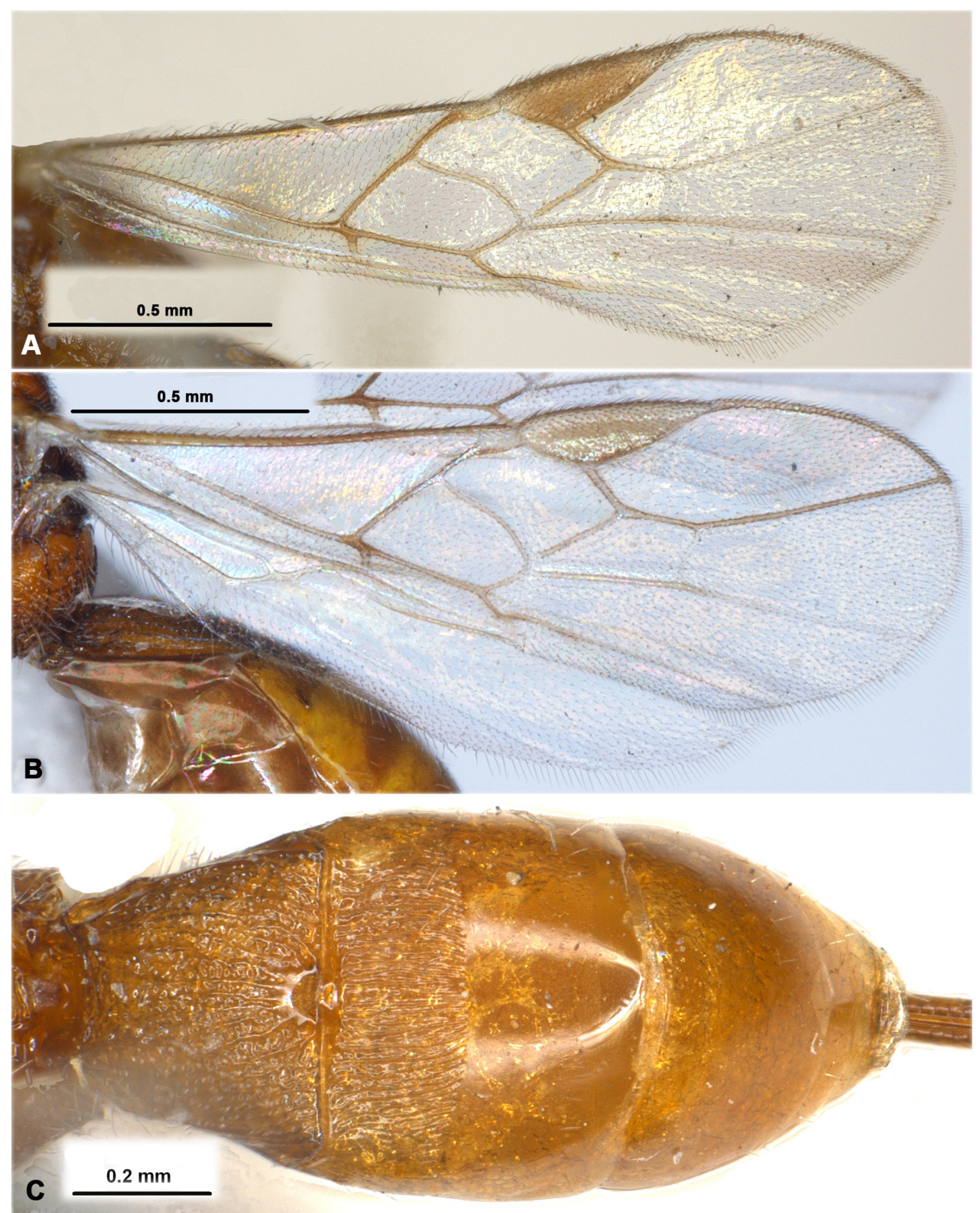

Fig. 35. Parallorhogas testaceus (Szépligeti, 1914). A, C. Holotype, o (MNBG). B. Arabian specimen (RMNH). A. Fore wing. B. Wings. C. Metasoma, dorsal view. 
SCULPTURE AND PUBESCENCE. Vertex and frons entirely and rather distinctly densely and evenly curvedly subaciculate; face almost entirely distinctly transversely striate with dense rugulosity or granulation between striae; temple mainly smooth. Mesoscutum densely and finely granulate-reticulate, rugulose in rather narrow area in medio-posterior half. Scutellum finally reticulate-coriaceous. Mesopleuron transversely striate in upper $0.4-0.5$, smooth or almost smooth in lower half. Metapleuron entirely densely rugose-reticulate. Propodeum with basolateral areas irregularly delineate by undulate carinae, smooth or almost smooth in anterior $0.3-0.5$ and rugulose on remaining part; areola rather large, indistinctly delineated by carinae, sparse rugose-reticulate to striate; remaining part of propodeum rather coarsely rugose-striate. Hind coxa mainly smooth, dorsally more or less distinctly and curvedly rugose-striate with granulation partly. Hind femur mostly finely reticulate-coriaceus with fine and dense striation dorsally. First metasomal tergite entirely coarsely striate, with dense rugulosity between striae, with two distinct and weakly convergent posteriorly dorsal carinae. Second tergite almost entirely densely striate, almost without or sometimes with weak rugulosity between striae, smooth or almost smooth laterally. Remaining tergites (including third one) entirely smooth. Vertex mainly with sparse, short and semi-erect yellowish setae, sometimes partly glabrous medially. Mesoscutum almost entirely with rather dense, short and semi-erect yellow setae. Mesopleuron medially widely glabrous. Hind tibia dorsally with short, more or less dense and semi-erect setae, their length 0.3-0.6 times maximum width of tibia.

Colour. Head brownish yellow or light reddish brown, infuscate dorsally. Mesosoma light reddish brown to yellowish brown at least partly. Metasoma mainly brownish yellow, first tergite reddish brown and sometimes with yellow tint. Antenna brown to dark brown, three basal segments yellow to brownish yellow. Palpi yellow or pale yellow. Legs mainly yellow or brownish yellow. Ovipositor sheath dark brown to black. Fore wing hyaline. Pterostigma brown, pale brown basally and apically.

\section{Male \\ Unknown. \\ Distribution}

Togo, *UAE.

Genus Hemidoryctes Belokobylskij, 1992

\section{Type species}

Hemidoryctes soror Belokobylskij, 1992 (= Heterospilus carbonarius Ashmead, 1894), by original designation.

\section{Remarks}

This is a monotypic genus whose type species has a pantropical distribution.

\section{Distribution}

Afrotropical, Australasian, Nearctic, Neotropical, Oceanic and Oriental regions.

Hemidoryctes carbonarius postfurcalis Belokobylskij subsp. nov. urn:1sid:zoobank.org:act:96D36DAE-6A4D-48FC-AC74-4898F18C9229

Figs 36-37

\section{Comparative diagnosis}

This new subspecies is very similar to the trans-tropical Hemidoryctes carbonarius carbonarius (Ashmead, 1894), but differs from it by the distinctly postfurcal position of the fore wing recurrent vein 
(m-cu) (always antefurcal in H. c. carbonarius) and recorded only in Yemen (with wide pantropical distribution for H. c. carbonarius).

\section{Etymology}

Named after the postfurcal position of the recurrent vein $(\mathrm{m}-\mathrm{cu})$ in the fore wing.

\section{Material examined}

\section{Holotype}

YEMEN • +; "Yemen, Al Kowd, i.2001, light tr., no. 5482, A.v. Harten \& S. Al Haruri, RMNH'01"; RMNH.

\section{Paratype}

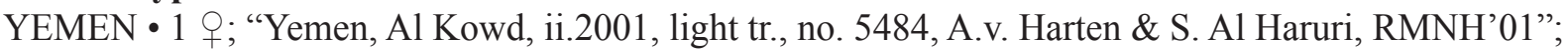
RMNH.

\section{Description}

\section{Female}

MeAsurements. Body length 3.9-4.1 mm; fore wing length $2.8-3.0 \mathrm{~mm}$.

HEAD. Head width (dorsal view) 1.4-1.5 times its median length, 1.2 times width of mesoscutum. Head behind eyes (dorsal view) weakly convex-roundly narrowed. Transverse diameter of eye 1.4-1.5 times longer than temple. Ocelli arranged in triangle with base 1.3 times its sides; POL 1.6-2.0 times Od, $0.5-0.6$ times OOL. Eye glabrous, 1.2 times as high as broad. Malar space $0.50-0.55$ times eye height, 0.9-1.0 times basal width of mandible. Face width almost equal to eye height and 1.3 times height of face and clypeus combined. Malar suture absent. Hypoclypeal depression subround, its width 0.6-0.7 times distance from edge of depression to eye, 0.4 times width of face. Occipital carina joined with hypostomal carina on short distance upper base of mandible. Hypostomal flange rather narrow.

AntenNa. Antenna slender, filiform, 25-27-segmented, 0.9 times as long as body. Scape 1.5-1.7 times longer than maximum width, 2.2-2.7 times longer than pedicel. First flagellar segment 5.8-6.5 times longer than its apical width, 1.1 times longer than second segment. Penultimate segment 4.0-4.2 times longer than wide, 0.5 times as long as first flagellar segment, about as long as apical segment; latter almost obtuse apically.

Mesosoma. Length 1.8 times its height. Neck of prothorax short, with distinct pronotal keel in anterior 0.3. Mesoscutum (lateral view) distinctly and almost perpendicularly elevated above pronotum; rather long (dorsal view), its median length 1.1 times maximum width. Notauli distinct, complete, rather wide, deep in anterior half and shallow in posterior half, crenulate-rugulose. Median lobe of mesoscutum (dorsal view) distinctly protruding, weakly convex anteriorly, without median furrow and anterolateral corners. Prescutellar depression (scutal sulcus) deep, distinctly rugulose, almost without straight carinae, 0.35-0.40 times as long as scutellum. Scutellum convex, with lateral carinae. Subalar depression rather shallow, rather wide, rugose-reticulate. Precoxal sulcus rather deep, almost straight, finely coriaceous anteriorly and smooth on posterior part, running along anterior 0.6 of lower part of mesopleuron. Metascutal lobe (lateral view) short and rounded distally. Metapleural flange wide, short, subrounded distally. Propodeum with small lateral tubercles.

Wings. Length of fore wing 3.6-3.8 times its width. Metacarpus (1-R1) 1.0-1.1 times as long as pterostigma. Radial vein (r) arising from middle of pterostigma. Second radial abscissa (3-SR) 2.5-2.7 times longer than first abscissa (r), 0.6-0.7 times as long as weakly concave third abscissa (SR1), 1.5 times longer than first radiomedial vein (2-SR). Second radiomedial (submarginal) cell long, 3.5-3.8 


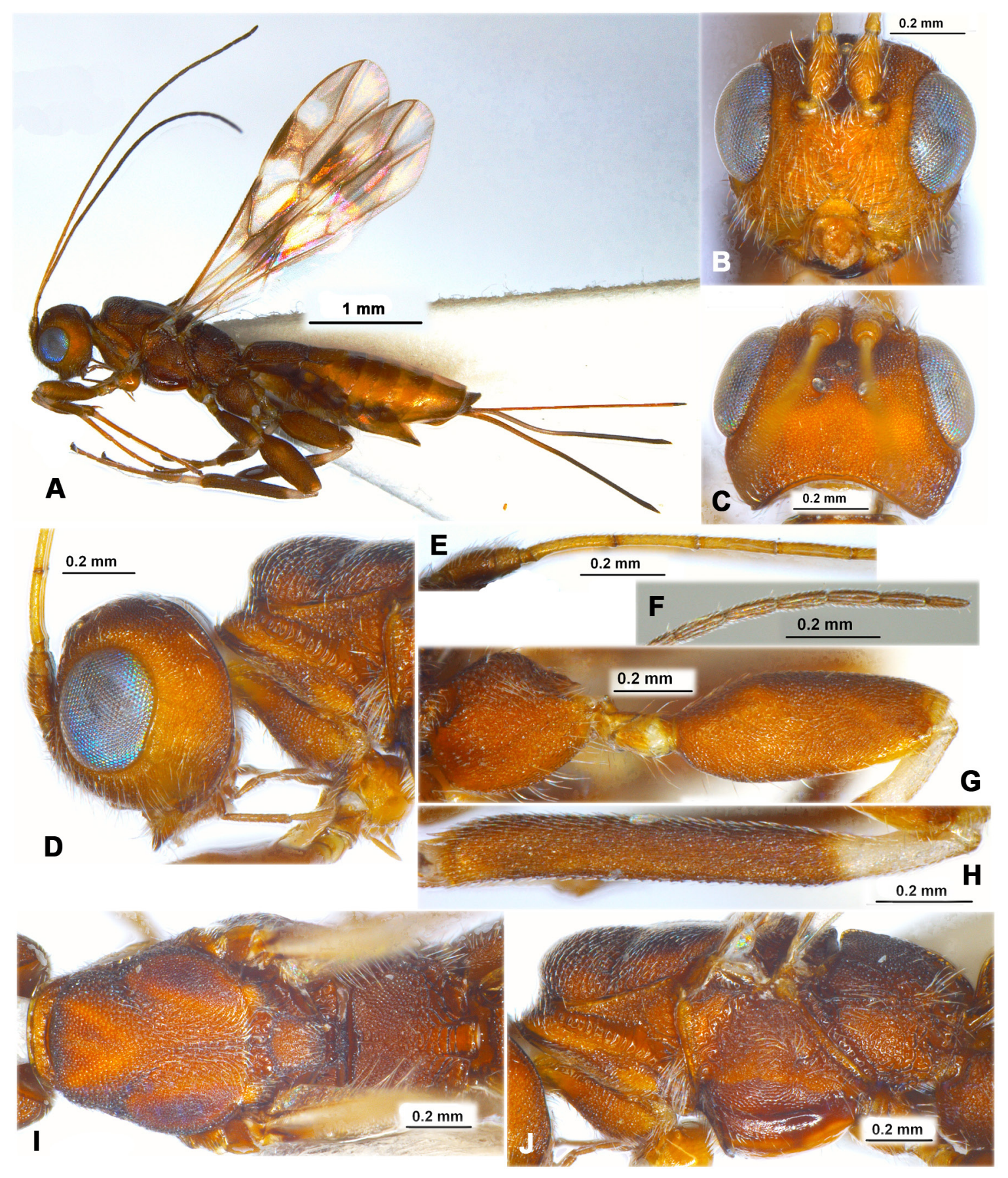

Fig. 36. Hemidoryctes carbonarius postfurcalis Belokobylskij subsp. nov., holotype, $q$ (RMNH). A. Habitus, lateral view. B. Head, front view. C. Head, dorsal view. D. Head and anterior part of mesosoma, lateral view. E. Basal segments of antenna. F. Apical segments of antenna. G. Hind coxa and femur. H. Hind tibia. I. Mesosoma, dorsal view. J. Mesosoma, lateral view. 

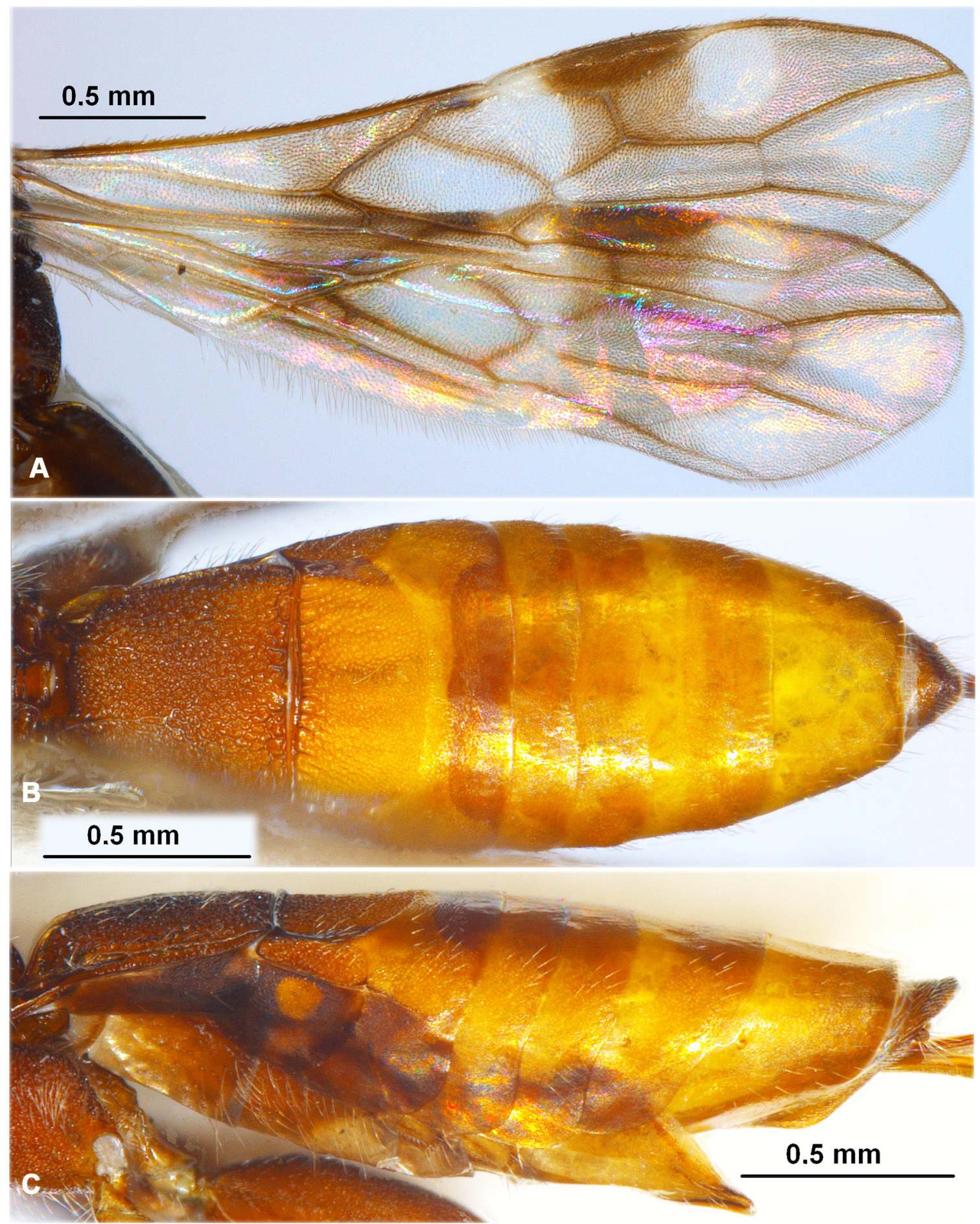

Fig. 37. Hemidoryctes carbonarius postfurcalis Belokobylskij subsp. nov., holotype, $q$ (RMNH). A. Wings. B. Metasoma, dorsal view. C. Metasoma, lateral view. 
times longer than its maximum width, 1.4 times longer than the narrow brachial (subdiscal) cell. First medial abscissa (1-SR+M) distinctly sinuate. Recurrent vein (m-cu) distinctly postfurcal, 4.5-5.0 times longer than second abscissa of medial vein $(2-\mathrm{SR}+\mathrm{M}), 0.6$ times longer than first radiomedial vein $(\mathrm{r}-$ $\mathrm{m})$. Nervulus (cu-a) strongly postfurcal, distance from nervulus (cu-a) to basal vein (1-M) 1.6-2.0 times nervulus (cu-a) length. Brachial (subdiscal) cell open apically, but present short brachial vein (CU1b). Parallel vein (CU1a) weakly curved basally. Hind wing 5.0-5.2 times longer than wide. First abscissa of costal vein $(\mathrm{C}+\mathrm{SC}+\mathrm{R})$ about as long as second abscissa. Medial (basal) cell rather narrow, weakly widened posteriorly, 11.0-13.0 times longer than wide, 0.4 times as long as hind wing. First abscissa of mediocubital vein (1-SC+R) 0.6 times as long as second abscissa (2-SC+R). Recurrent vein (m-cu) straight, oblique to base of wing, interstitial, sclerotised.

Legs. Hind coxa large, dorsally with wide and high lump, 1.2-1.3 times longer than maximum high. Hind femur 2.6-2.9 times longer than wide. Hind tibia weakly thickened. Hind tarsus 1.1 times longer than hind tibia. Hind basitarsus 0.7 times as long as second-fifth segments combined. Second segment of hind tarsus $0.55-0.60$ times as long as basitarsus, 1.7- 1.9 times longer than fifth segment (without pretarsus).

Metasoma. Metasoma 1.2-1.3 times longer than mesosoma and head combined. First metasomal tergite with small spiracular protuberances in anterior 0.3 , distinctly and almost linearly widened from base to subapex, than weakly narrowed. Length of first tergite 1.00-1.05 times its posterior width, 1.3-1.5 times length of propodeum; posterior width about 2.0 times its minimum anterior width. Second tergite with not high longitudinal sublateral carinae. Median length of second tergite 0.5 times its anterior width, 1.5 times length of third tergite. Second suture shallow but distinctly, strongly convex medially, with rather deep sublateral breaks. Third tergite without transverse furrow. Ovipositor sheath $0.85-0.90$ times as long as metasoma, 1.4-1.5 times longer than mesosoma, 0.6-0.7 times as long as fore wing.

SCUlPTURE AND PUBESCENCE. Vertex and frons entirely distinctly and densely granulate-reticulate; face entirely distinctly curvedly transversely striate with dense rugulosity between striae; temple mainly granulate-reticulate, in lower half with oblique subtransverse striae. Mesoscutum densely and small granulate-reticulate, rugulose in rather narrow area in medio-posterior half. Scutellum entirely granulate-reticulate. Mesopleuron densely rugulose-reticulate in upper 0.5 , finely coriaceous to almost smooth partly in lower half. Metapleuron entirely densely reticulate-areolate, areolae smaller and dense reticulate-areolate in anterior third. Propodeum with basolateral areas delineated by rather fine carinae, with long and narrow areola finely delineated by carinae; with short and distinct basomedian carina in anterior quarter or third; propodeum in anterior half densely and small reticulate-areolate, sparsely rugose-striate in posterior half. Hind coxa entirely densely reticulate-granulate, dorsally with additional rugosity. Hind femur entirely very densely and small reticulate-areolate. First metasomal tergite entirely distinctly rugulose-reticulate, with two distinct and weakly convergent posteriorly dorsal carinae in anterior quarter. Second tergite almost entirely with distinct and divergent posteriorly striae and with dense rugulosity between striae, densely reticulate granulate laterally. Third tergite finely to very finely and densely semi-circularly transversely aciculate in anterior half, reticulate coriaceous in latero-posteriorly, almost smooth on wide medial half. Remaining tergites mainly smooth, but densely and finely or very finely granulate laterally. Vertex mainly with sparse, short and semi-erect white setae directed forward. Mesoscutum entirely with dense, short and semi-erect white setae. Mesopleuron medially widely glabrous. Hind coxa dorsally with long and dense adpressed white setae. Hind tibia dorsally with very short, dense and semi-erect white setae, their length about 0.2 times maximum width of tibia.

Colour. Head and most part of prothorax brownish yellow, reddish brown to dark reddish brown dorsally. Mesosoma mainly reddish brown or dark reddish brown, infuscate dorsally, yellow or brownish yellow ventrally. Metasoma mainly brownish yellow to yellow, first and second or sometimes also third 
tergites reddish brown, remaining tergites medio-posteriorly infuscate; sternite in upper halves or thirds dark reddish brown. Antenna brown to dark brown in apical half, eight-ten basal segments yellow to brownish yellow. Palpi reddish brown, yellow distally. Legs mainly reddish brown to dark reddish brown partly; fore and middle coxae brownish yellow; all tarsi yellow or pale brown with infuscate middle segments; all tibiae and basitarsi basally white at rather long distance. Ovipositor sheath brown in anterior half and black in posterior half. Fore wing maculate, hyaline basally. Pterostigma dark brown, pale yellow to whitish in basal third and apically.

Male

Unknown.

\section{Distribution}

Yemen.

Tribe Heterospilini Fischer, 1981

Genus Heterospilus Haliday, 1936

\section{Type species}

Rogas quaestor Haliday, 1836, by monotypy.

\section{Remarks}

This is one of the largest genera in the subfamily Doryctinae, with most of its species being distributed in the Neotropical region (Marsh et al. 2013). Heterospilus consists of two subgenera, Eoheterospilus Belokobylskij \& Maetô, 2009 and Heterospilus s. str.

\section{Distribution}

Afrotropical, Australasian, Nearctic, Neotropical, Oceanic, Oriental and Palaearctic regions.

Heterospilus (Eoheterospilus) rubrocinctus (Ashmead, 1905)

Fig. 38

Hecabolus rubrocinctus Ashmead, 1905: 8.

Heterospilus rubrocinctus - Belokobylskij 1994: 23. — Belokobylskij \& Maetô 2009: 175. — Tang et al. 2013: 2005. - Yu et al. 2016.

\section{Material examined}

UNITED ARAB EMIRATES • 2 + $O$; "United Arab Emirates, Fujairah (3983), light tr., 2-30.i.2006, $25^{\circ} 08^{\prime}$ N, 56 $21^{\prime}$ E, A.v. Harten, RNMH'06”; RMNH • 1 q: same label data as for preceding; ZISP • 1 ㅇ; "United Arab Emirates, Fujairah (4117), light tr., 28.ii-1.iv.2006, $25^{\circ} 08^{\prime} \mathrm{N}, 56^{\circ} 21^{\prime}$ E, A.v. Harten, RNMH'06"; RMNH • 1 ơ; "United Arab Emirates, Fujairah (6440), light tr., 29.xi.2005-2.i.2006, $25^{\circ} 08^{\prime} \mathrm{N}, 56^{\circ} 21^{\prime}$ E, A.v. Harten, RNMH'06”; RMNH.

YEMEN - 2 qo; "Yemen (7189), Al Kadan, v.2002, Light trap, A.v. Harten \& A.R. Al Yarimi. RMNH'02"; RMNH • 1 § ; "Yemen, Al Kowd, ii.2000, light tr., no 4431, A.v. Harten \& S. Al Haruri, RMNH'01"; RMNH.

\section{Distribution}

China, Japan, Philippines, Russia (south of Far East), Vietnam, *UAE, *Yemen. 


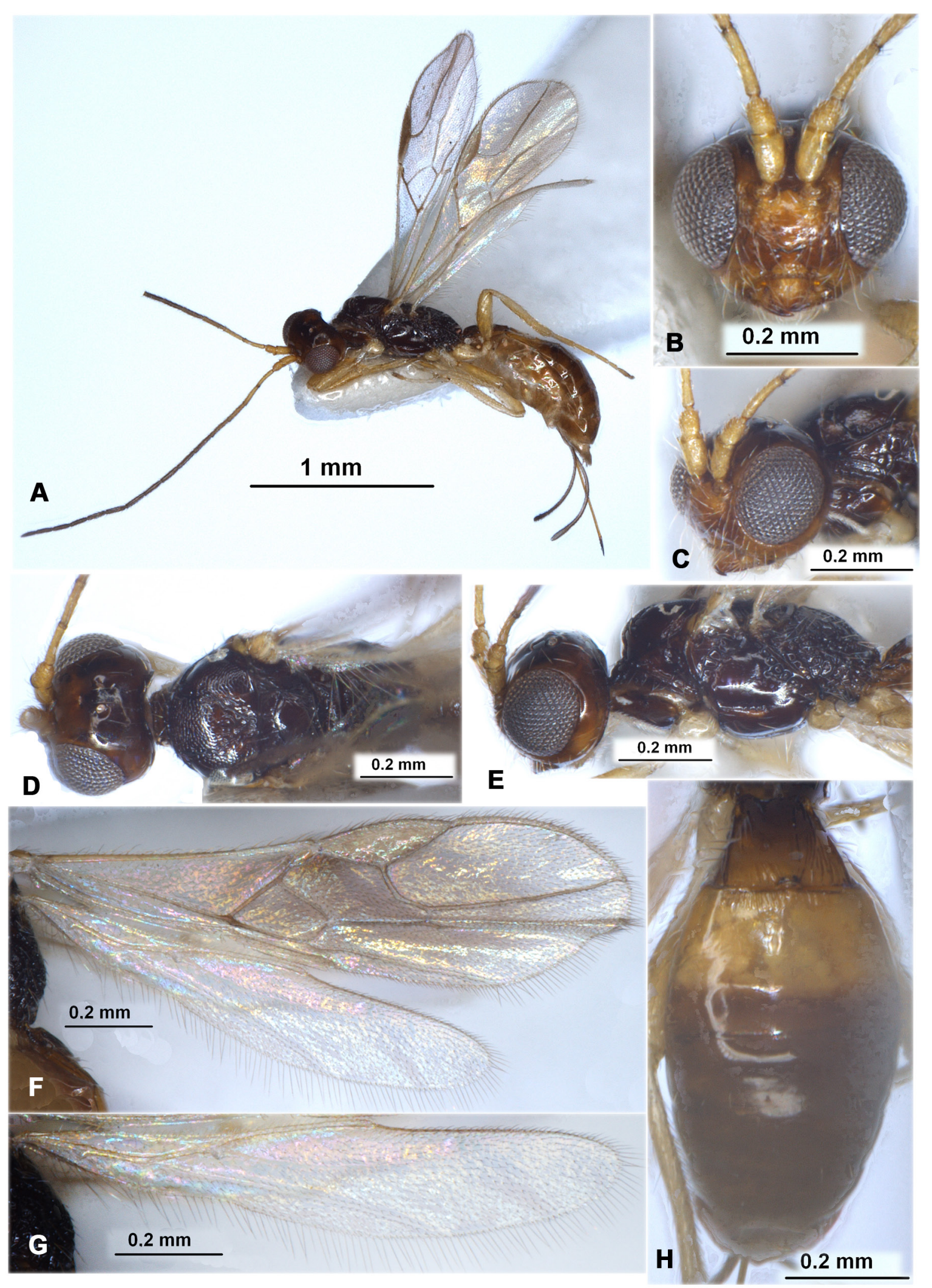

Fig. 38. Heterospilus (Eoheterospilus) rubrocinctus (Ashmead, 1905), (RMNH). A. Habitus, lateral view. B. Head, front view. C. Head, antero-lateral view. D. Head and mesosoma, dorsal view. E. Head and mesosoma, lateral view. F. Wings. G. Hind wing. H. Metasoma, dorsal view. 
Genus Neoheterospilus Belokobylskij, 2006

\section{Type species}

Neoheterospilus koreanus Belokobylskij, 2006, by original designation.

\section{Remarks}

This is a relatively small and widely distributed genus, which consists of two subgenera, the Neotropical Harpoheterospilus Belokobylskij, 2006, and the Old World (including Australia) Neoheterospilus s. str.

\section{Distribution}

Afrotropical, Australian, Neotropical, Oriental and Palaearctic regions.

Neoheterospilus (Neoheterospilus) alkowdi Belokobylskij, 2020

Neoheterospilus alkowdi Belokobylskij, 2020a: 592.

Neoheterospilus sp. (ð) - Edmardash et al. 2020: 54.

\section{Material examined}

\section{Holotype}

YEMEN • +; "Yemen (4055), Al Kowd, ix.1999, light-trap, A.v. Harten \& S. Al Haruri, RMNH'00"; RMNH.

\section{Paratypes}

YEMEN • 1 q; "Yemen (4054), Al Kowd, viii.1999, light-trap, A.v. Harten \& S. Al Haruri, RMNH'00"; ZISP • 1 9; "Yemen, Al Kowd, v-vi.2000, light-tr, no 4719, A.v. Harten \& S. Al Haruri, RMNH'01"; RMNH • 1 ऊे; "Yemen, Al Kowd, ix.2003, light trap; A.v. Harten \& S. Al Haruri, RMNH'03"; RMNH - 1 9; "Yemen (5404), Hammam 'Ali, from coffee-berries (with Ceratitis capitata ?), 14.iii.2001, A.v. Harten, RMNH'02"; RMNH.

\section{Distribution}

Yemen.

Neoheterospilus (Neoheterospilus) yemenus Belokobylskij, 2020

Neoheterospilus yemenus Belokobylskij, 2020a: 595.

\section{Material examined}

\section{Holotype}

YEMEN • P. "Yemen (5700), Ar Rujum, 9.iv-5.vi.2001, Mal. trap, A.v. Harten, RMNH'02”; RMNH.

\section{Distribution}

Yemen. 
Tribe Rhaconotini Fahringer, 1928

Genus Platyspathius Viereck, 1911

\section{Type species}

Platyspathius pictipennis Viereck, 1911, by original designation.

\section{Remarks}

This is relatively small and widely distributed genus, though it mainly occurs in tropical and subtropical climatic zones. It consists of two subgenera, the monotypic Malagasy Lenticularia van Achterberg, 2003 and Platyspathius s. str.

\section{Distribution}

Afrotropical, Australasian, Oceanic, Oriental and Palaearctic regions.

Platyspathius (Platyspathius) brevis Belokobylskij sp. nov. urn:lsid:zoobank.org:act:B90DA11E-9AE7-404F-A113-2DF94A946662

Figs $39-40$

\section{Comparative diagnosis}

This new species is similar to Platyspathius clymene Nixon, 1943 from South Africa and Congo, but it differs from the latter by having the first and second metasomal tergites short (longer in P. clymene), second suture practically without crenulation or striation (with distinct striation in P. clymene), the sculpture of the mesosoma fine (coarse in $P$. clymene), fore and middle coxae yellowish brown (dark reddish brown in P. clymene).

\section{Etymology}

This species is named after the Latin word 'brevis' (short), because it has a short second metasomal tergite.

\section{Material examined}

Holotype

YEMEN • Q; "Yemen (6141), Al Kowd, 16-20.viii.2001, light trap, A.v. Harten \& S. Al Haruri. RMNH'02"; RMNH.

\section{Paratypes}

YEMEN - 1 q; "Yemen (5750), Al Kowd, 8-12.vii.2001, light trap, A.v. Harten \& S. Al Haruri. RMNH'02"; RMNH • 1 क , 1 §;; "Yemen (5404), Hammam "Ali, from coffee-berries (with Ceratitis capitata ?), 14.iii.2001, A.v. Harten, RMNH'02”; RMNH.

\section{Description}

\section{Female}

MEASUREMENTS. Body length 3.1-3.9 mm; fore wing length 1.6-2.2 mm.

HEAD. Head width (dorsal view) 1.4-1.5 times its median length, 1.2 times width of mesoscutum. Vertex distinctly convex. Head behind eyes (dorsal view) weakly and evenly convex in anterior half and weakly roundness decreased in posterior half. Transverse diameter of eye (dorsal view) 1.3 times longer than temple. Ocelli small, with ocellar triangle base 1.2 times its sides. POL 2.3-2.5 times Od, 0.5-0.7 times OOL. Eye glabrous, in lateral view oblique, its maximum diameter 1.3 times minimum diameter. Malar 
space 0.6 times maximum diameter of eye, 1.0-1.1 times basal width of mandible. Face convex, with rather distinct and narrow convex vertical stripe; width of face 1.0-1.1 times maximum diameter of eye, 1.4-1.5 times height of face and clypeus combined. Clypeal suture complete, distinct laterally and very fine dorsally. Dorsal margin of clypeus situated distinctly lower than lower level of eyes; ventral margin of clypeus with narrow flange. Hypoclypeal depression medium-sized and rounded, its width 0.6 times shortest distance from edge to eye, 0.4 times width of face. Occipital carina dorsally complete and almost not broken toward ocellar triangle, not reaching below hypostomal carina and obliterated rather long distance before mandible base. Hypostomal flange very narrow.

AnTENNA. Antenna slender, weakly thickened towards apex, 20-23-segmented, about 0.7-0.8 times as long as body. Scape 1.3-1.5 times longer than maximum width. First flagellar segment 4.3-4.8 times longer than apical width, about 1.1 times longer than second segment. Penultimate segment 2.4-3.0 times longer than width, 0.7 times as long as first segment, 0.9 times as long as apical segment; latter weakly acuminate apically and without spine.

Mesosoma. Mesosoma not depressed, its maximum length 1.8-1.9 times its maximum height. Pronotal carina rather fine but distinct, its anterior branch situated submedially, posterior branch widely connected with anterior margin of pronotum. Pronotal lateral depression rather wide and shallow, not delineated by carinae. Mesoscutum (lateral view) distinctly, obliquely and curvedly elevated above pronotum, its median lobe (dorsal view) distinctly convex anteriorly and without anterolateral corners, without longitudinal median furrow; mesoscutum in dorsal view $0.90-0.95$ times as long as wide. Notauli complete, rather distinct, but shallow posteriorly, narrow, but weakly widened posteriorly, distinctly crenulate, fused posteriorly. Prescutellar depression rather deep, short medially, with distinct median carina, with several additional carinae, $0.30-0.35$ times as long as scutellum. Scutellum convex, with fine and complete lateral carinae, 1.0-1.1 times longer than maximum anterior width. Subalar depression rather narrow, shallow, finely reticulate, without rugosity, with sparse crenulation below. Precoxal sulcus distinct, rather short, straight, oblique, finely crenulate, running along anterior half of lower part of mesopleuron. Postpectal carina absent. Metanotum with distinct, rather wide and distally rounded transparent medio-dorsal lobe (lateral view). Metapleural flange (lobe) narrow, short, subpointed distally. Propodeum weakly convex, without lateral tubercles.

Wings. Fore wing 3.8-4.0 times longer than wide. Pterostigma subtriangular and rather widened, 3.64.2 times longer than its maximum width. Radial vein (r) arising weakly before middle of pterostigma. Radial (marginal) cell not shortened, metacarpus (1-R1) 1.15-1.20 times longer than pterostigma. Second radial abscissa (3-SR) 3.2-3.4 times longer than first abscissa (r) and forming with it obtuse angle, $0.70-0.75$ times as long as very weakly curved third abscissa (SR1), 1.2-1.3 times longer than straight first radiomedial vein (2-SR). Second radiomedial (submarginal) cell not narrowed distally, its length 3.1-3.5 times maximum width, 1.5-1.6 times length of rather wide brachial (subdiscal) cell. Second abscissa of medial vein (2-SR+M) long, 0.7-0.9 times as long as recurrent vein (m-cu). Nervulus (cu-a) weakly postfurcal, declivous, distinctly slanting to base of wing. Mediocubital vein (M+CU1) strongly sinuate and in posterior half deeply curved to longitudinal anal vein (1-1A). Widened brachial (subdiscal) cell rather evenly curvedly closed apically weakly before or on level of recurrent vein (m-cu). Parallel vein (CU1a) interstitial. Hind wing 4.5-4.9 times longer than its maximum width. First costal abscissa $(\mathrm{C}+\mathrm{SC}+\mathrm{R})$ 0.7-0.8 times as long as second abscissa (1-SC+R). First abscissa of mediocubital vein $(\mathrm{M}+\mathrm{CU})$ 0.6-0.7 times as long as second abscissa (1-M). Recurrent vein (m-cu) rather long, unsclerotised and not pigmented, interstitial, strongly oblique towards base of wing.

LEGs. Fore tibia anterior surface with distinct, thick, rather sparse spines arranged in wide stripe. Segments of middle tarsus longer than their width. Hind coxa rather short, subtriangular, with distinct basoventral corner and indistinct tubercle, 1.4-1.5 times longer than maximum width. Hind femur rather 


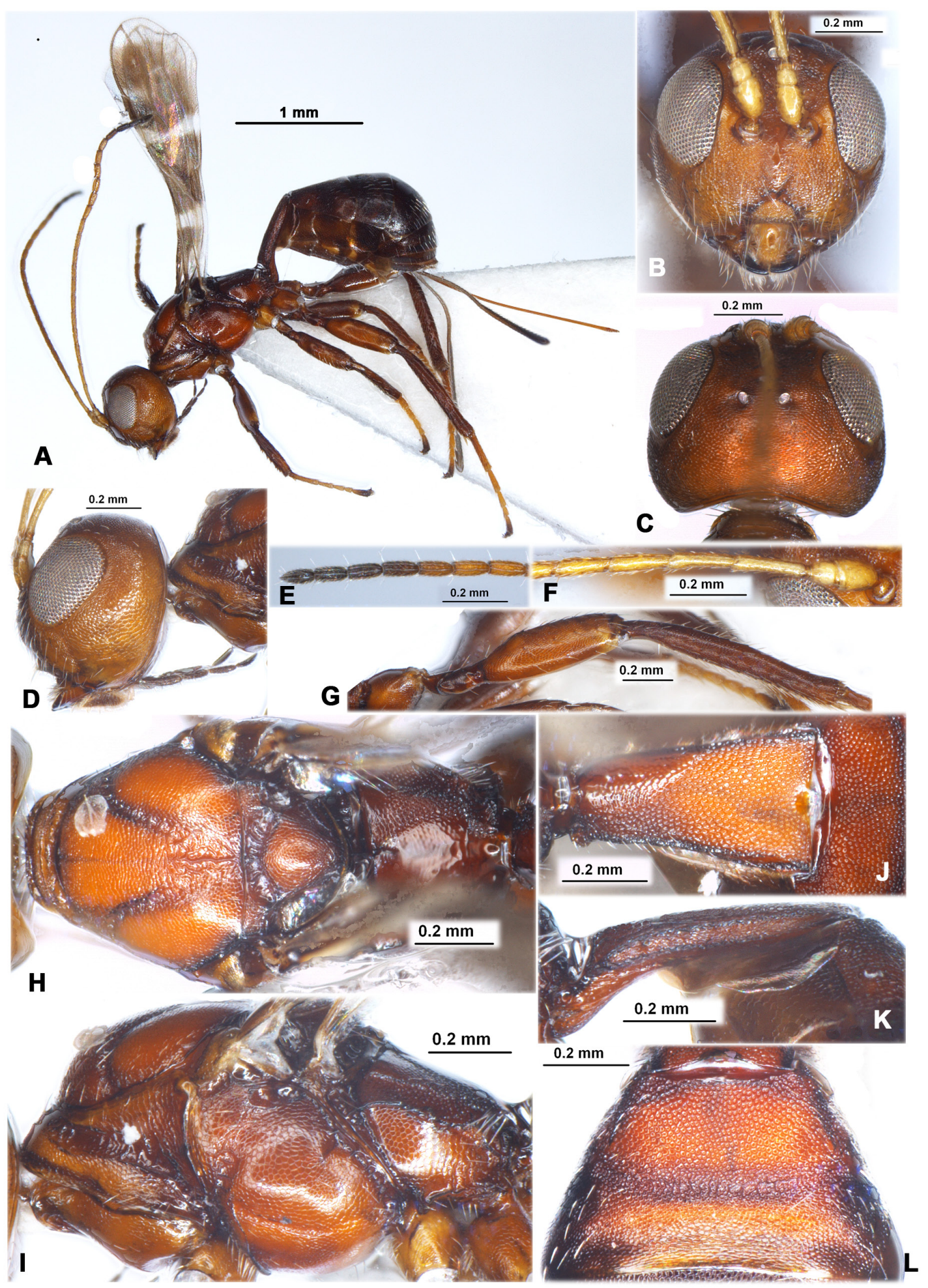

Fig. 39. Platyspathius (P.) brevis Belokobylskij sp. nov., holotype, $\odot$ (RMNH). A. Habitus, lateral view. B. Head, front view. C. Head, dorsal view. D. Head, lateral view. E. Apical segments of antenna. F. Basal segments of antenna. G. Hind leg. H. Mesosoma, dorsal view. I. Mesosoma, lateral view. J. First metasomal tergite, dorsal view. K. First metasomal tergite, lateral view. L. Second and third metasomal tergites, dorsal view. 
wide, with dorsal wide tubercle in anterior quarter, elongate-oval, 3.0-3.4 times longer than wide. Hind tibia with four-five slender spines on outer distal margin. Hind tarsus 0.90-0.95 times as long as hind tibia. Hind basitarsus $0.55-0.60$ times as long as remaining segments combined. Second segment of hind tarsus 0.6 times as long as basitarsus, $1.20-0.25$ times longer than narrow fifth segment (without pretarsus). Tarsal segments thickened. Claws short, thick basally, with short and distinctly curved its posterior part.

Metasoma. Metasoma rather short. Petiole (lateral view) ventrally distinctly curved, dorsally arched in anterior fifth and weakly curved on remaining part, the most high in anterior fifth; in dorsal view, petiole rather wide, weakly, evenly and linearly widened toward apex of petiole, with small spiracular tubercles in anterior quarter, with small dorsope. Length of petiole 1.8-2.1 times its posterior width, 1.7-1.9 times length of propodeum; posterior width of prtiole 2.0-2.1 times minimum anterior width. Second to sixth tergites with laterotergites separated; second and third separated laterotergites perhaps fused at least partly. Second suture very shallow, rather wide, weakly evenly curved, densely reticulate, practically without crenulation. Second tergite short, its median length 0.6-0.7 times its anterior width, 1.4-1.6 times length of third tergite. Median length of second and third tergites combined 0.9-1.1 times its anterior width, $0.50-0.55$ times their maximum width. Ovipositor straight. Ovipositor sheath 2.0-2.5
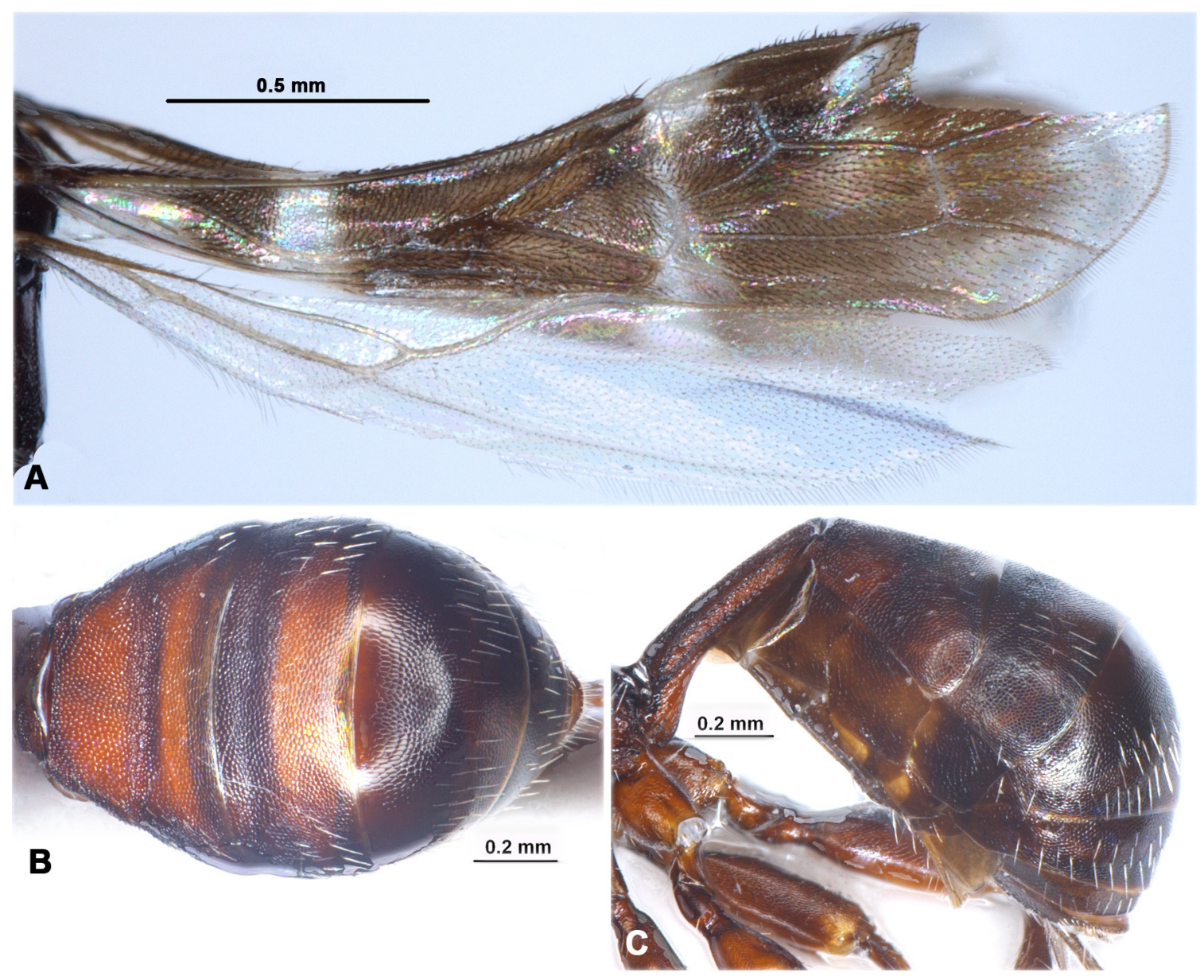

Fig. 40. Platyspathius (P.) brevis Belokobylskij sp. nov., holotype, $q$ (RMNH). A. Wings. B. Metasoma without first tergite, dorsal view. C. Metasoma, lateral view. 
times longer than petiole, $0.7-0.8$ times as long as metasoma, 1.3-1.5 times longer than mesosoma, $0.75-0.90$ times as long as fore wing.

SCUlPtURE AND PUBESCENCE. Head mainly densely reticulate-coriaceous, frons additionally with dense rugulosity; face additionally partly with granulation, medially almost smooth or weakly granulate in narrow vertical stripe. Mesoscutum almost entirely densely reticulate-coriaceous with fine reticulation and short longitudinal carina in medio-posterior third; scutellum very finely reticulate-coriaceous; mesopleuron very finely reticulate-coriaceous. Propodeum without areas, with long and distinct lateral carinae and short medial carina; entirely densely rugose-reticulate, finely reticulate to almost smooth anteriorly. Hind coxa and femur entirely densely and very finally reticulate-coriaceous. First to third tergites almost entirely densely and distinctly reticulate-areolate, coriaceous in posterior third of third tergite; fourth to sixth tergites entirely and finally reticulate-coriaceous. Vertex mainly glabrous, with very sparse short pale setae laterally. Mesoscutum mainly glabrous, with short and sparse white setae narrowly arranged along notauli and laterally. Setae of hind tibia semi-erect, short and rather sparse, their length on dorsal surface $0.4-0.5$ times maximum width of tibia.

Colour. Body mainly dark reddish brown or reddish brown, head at most part (except dark dorsal part) and usually propleura yellowish brown or light reddish brown. Palpi dark brown. Antenna mainly brownish yellow to yellow, four-five apical segments dark brown to black. Legs mainly dark reddish brown, fore and middle coxae yellowish brown; hind tibia basally always same colour as remaining parts of tibia; all tarsi mainly light reddish brown. Ovipositor sheath mainly brown to dark brown, pale brown anteriorly. Fore wing strongly darkened at most part, with contrasting rather small hyaline or almost white spots and stripes (Fig. 40A), with rather wide transverse hyaline stripe cross narrowest part of submedial cell. Pterostigma mainly dark brown, white in basal 0.3 .

\title{
Male
}

Body length $2.8 \mathrm{~mm}$; fore wing length $1.6 \mathrm{~mm}$. Transverse diameter of eye (dorsal view) almost as long as temple. Antenna 20 -segmented. First flagellar segment as long as second segment. Maximum length of mesosoma 2.0 times its maximum height. Mesoscutum in dorsal view as long as wide. Hind femur more slender, 3.5 times longer than wide. Hind tibia distinctly widened, especially medially, its maximum width almost 0.9 times femur width. Hind tarsus slender. Petiole more narrow. Second tergite weakly longer, its median length 0.9 times anterior width, 1.3 times length of third tergite. Median length of second and third tergites combined 1.5 times its anterior width, 0.7 times their maximum width. Otherwise similar to female.

\section{Distribution}

Yemen.

\author{
Platyspathius (Platyspathius) longicaudis Belokobylskij sp. nov. \\ urn:1sid:zoobank.org:act:032B842F-77E1-4F5F-8B72-5FD3E116DF4C
}

Figs $41-42$

\section{Comparative diagnosis}

This new species is similar to Platyspathius clymene Nixon, 1943 from South Africa and Congo, but it differs from latter by the following characters: frons distinctly densely transversely striate with reticulation (reticulate-rugulose in $P$. clymene), notauli rather deep impressed and wide (shallow and narrow in $P$. clymene), second metasomal tergite long (shorter in P. clymene), precoxal sulcus shallow and shorter (deeper and longer in P. clymene), second radial abscissa (3-SR) 0.9 times as long as third abscissa (SR1) (about 0.7 times in P. clymene), second metasomal suture finely crenulate (distinctly crenulate in P. clymene), ovipositor long, almost as long as body (distinctly shorter in P. clymene). 


\section{Etymology}

This species is named after the Latin word 'longis' (long) and 'cauda' (tail), due to its long ovipositor.

\section{Material examined}

\section{Holotype}

YEMEN - q; "Yemen (5962), 12 km NW Manakhah, Mal. trap; 3.vii-21.viii.2001, A.v. Harten, RNMH'02"; RMNH.

\section{Description}

Female

MeAsurements. Body length $4.5 \mathrm{~mm}$; fore wing length $2.5 \mathrm{~mm}$.

HeAD. Head width (dorsal view) 1.7 times its median length, 1.4 times width of mesoscutum. Vertex distinctly convex. Head behind eyes (dorsal view) rather distinctly and evenly convex in anterior half and weakly roundness decreased in posterior half. Transverse diameter of eye (dorsal view) 1.2 times longer than temple. Ocelli small, with ocellar triangle base 1.3 times its sides. POL 1.7 times Od, 0.5 times OOL. Eye glabrous, in lateral view oblique, its maximum diameter 1.3 times minimum diameter. Malar space 0.7 times maximum diameter of eye, 1.1 times basal width of mandible. Face convex, with distinct and narrow convex vertical stripe; width of face 1.2 times maximum diameter of eye, 1.45 times height of face and clypeus combined. Clypeal suture almost complete, distinct laterally and very fine dorsally. Dorsal margin of clypeus situated distinctly lower than lower level of eyes; ventral margin of clypeus with narrow flange. Hypoclypeal depression medium-sized and rounded, its width 0.55 times shortest distance from edge to eye, 0.35 times width of face. Occipital carina dorsally complete and distinctly but not strongly broken toward ocellar triangle, not reaching below hypostomal carina and obliterated short distance before mandible base. Hypostomal flange wide.

Antenna. Antenna slender, filiform, 28-segmented, 0.85 times as long as body. Scape 1.8 times longer than maximum width. First flagellar segment 5.3 times longer than apical width, almost as long as second segment. Penultimate segment about 3.0 times longer than width, 0.5 times as long as first segment, as long as apical segment; latter acuminate apically and without spine.

Mesosoma. Mesosoma not depressed, its maximum length 2.1 times its maximum height. Pronotal carina distinct, its anterior branch situated submedially, its posterior branch indistinct. Pronotal lateral depression wide and shallow, not delineated by carinae. Mesoscutum (lateral view) distinctly, but not high, obliquely and curvedly elevated above pronotum, its median lobe (dorsal view) distinctly convex anteriorly and without anterolateral corners, without longitudinal median furrow; mesoscutum in dorsal view 1.1 times longer than wide. Notauli complete, distinct, rather wide, shallow, distinctly crenulate, almost fused posteriorly. Prescutellar depression rather deep, short medially, with distinct median carina, densely and distinctly curvedly carinate, 0.3 times as long as scutellum. Scutellum strongly convex, with distinct and complete lateral carinae, as long as maximum anterior width. Subalar depression rather narrow, distinct, reticulate-rugose. Precoxal sulcus distinct, rather short, curved, crenulate, running along anterior half of lower part of mesopleuron. Postpectal carina absent. Metanotum with distinct, rather wide and distally rounded medio-dorsal lobe (lateral view). Metapleural flange (lobe) narrow, short, subrounded distally. Propodeum flat, without lateral tubercles.

Wings. Fore wing 4.1 times longer than wide. Pterostigma subtriangular and rather widened, 4.3 times longer than its maximum width. Radial vein (r) arising from middle of pterostigma. Radial (marginal) cell not shortened, metacarpus (1-R1) 1.2 times longer than pterostigma. Second radial abscissa (3-SR) 3.8 times longer than first abscissa (r) and forming with it almost straight angle, 0.9 times as long as the weakly curved third abscissa (SR1), 1.6 times longer than straight first radiomedial vein (2-SR). Second 


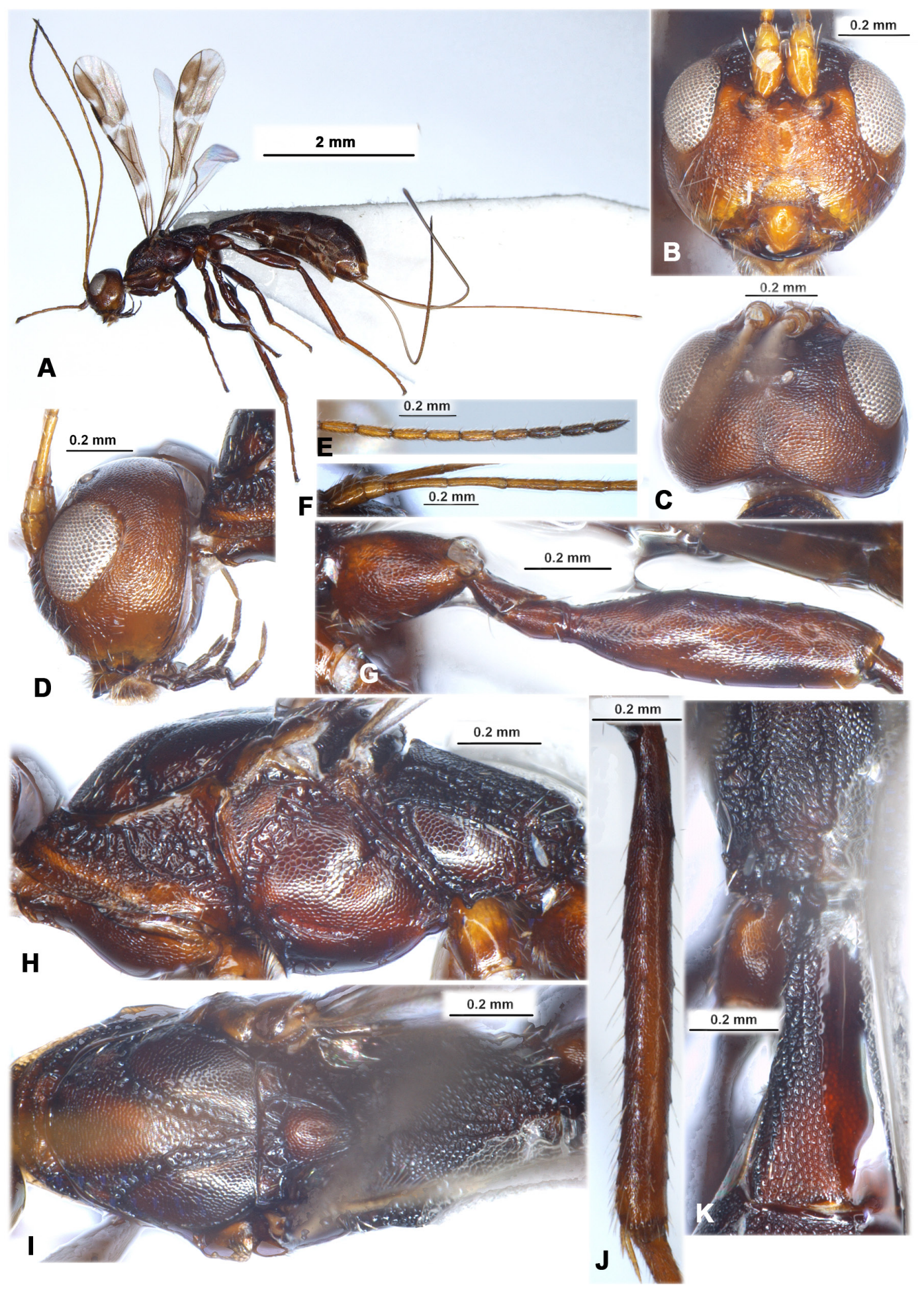

Fig. 41. Platyspathius (P.) longicaudis Belokobylskij sp. nov., holotype, $q$ (RMNH). A. Habitus, lateral view. B. Head, front view. C. Head, dorsal view. D. Head, lateral view. E. Apical segments of antenna. F. Basal segments of antenna. G. Hind leg. H. Mesosoma, lateral view. I. Mesosoma, dorsal view. J. Hind tibia. K. Propodeum and first metasomal tergite, dorsal view. 
radiomedial (submarginal) cell not narrowed distally, its length 3.8 times maximum width, 1.15 times length of narrow brachial (subdiscal) cell. Second abscissa of medial vein (2-SR+M) short, 0.4 times as long as recurrent vein (m-cu). Nervulus (cu-a) interstitial, declivous, strongly slanting to base of wing. Mediocubital vein $(\mathrm{M}+\mathrm{CU} 1)$ strongly sinuate and in posterior half deeply curved to longitudinal anal vein (1-1A). Narrow brachial (subdiscal) cell evenly curvedly closed apically on level of recurrent vein (m-cu). Parallel vein (CU1a) interstitial. Hind wing 5.4 times longer than its maximum width. First costal abscissa $(\mathrm{C}+\mathrm{SC}+\mathrm{R}) 0.8$ times as long as second abscissa $(1-\mathrm{SC}+\mathrm{R})$. First abscissa of mediocubital vein $(\mathrm{M}+\mathrm{CU}) 0.45$ times as long as second abscissa $(1-\mathrm{M})$. Recurrent vein $(\mathrm{m}-\mathrm{cu})$ short, unsclerotised and not pigmented, distinctly antefurcal, strongly oblique towards base of wing.

LeGs. Fore tibia anterior surface with short, thick, sparse spines arranged in wide stripe. Segments of middle tarsus considerably longer than their width. Hind coxa rather short, subtriangular, with rather distinct basoventral corner and with very small tubercle, 1.4 times longer than maximum width. Hind femur rather wide, with dorsal wide tubercle in anterior quarter, elongate-oval, 3.5 times longer than wide. Hind tibia with 5 slender spines on outer distal margin. Hind tarsus almost as long as hind tibia. Hind basitarsus 0.65 times as long as remaining segments combined. Second segment of hind tarsus 0.6 times as long as basitarsus, 1.8 times longer than narrow fifth segment (without pretarsus). Tarsal segments rather slender. Claws short, thick basally, with short and distinctly curved its posterior part.

Metasoma. Metasoma long. Petiole (lateral view) ventrally almost straight, dorsally arched in anterior quarter and almost straight on remaining part, almost same high; in dorsal view, petiole rather wide, evenly and linearly widened toward apex of petiole, with small spiracular tubercles in anterior third, without dorsope. Length of petiole 1.7 times its posterior width, 1.6 times length of propodeum; posterior width of petiole about 2.0 times minimum anterior width. Second to sixth tergites with laterotergites separated; second and third separated laterotergites not fused. Second suture shallow, rather wide, evenly curved, crenulate with reticulation. Second tergite long, its median length 1.1 times anterior width, 2.1 times length of third tergite. Median length of second and third tergites combined 1.6 times anterior width of second tergite, 1.1 times their maximum width. Ovipositor almost straight. Ovipositor sheath 5.0 times longer than petiole, 1.5 times longer than metasoma, almost 3.0 times as long as mesosoma, 2.5 times longer than fore wing.

SCULPTURE AND PUBESCENCE. Head mainly densely small reticulate-granulate or reticulate-areolate; frons additionally with distinct curved transverse striation; face medially narrow almost smooth. Mesoscutum almost entirely densely reticulate-coriaceous with reticulation in medio-posterior third, scutellum finely densely reticulate; mesopleuron mainly densely reticulate-coriaceous. Propodeum without areas, with rather long, fine, undulate medial carina in anterior half; entirely rugose-reticulate. Hind coxa and femur entirely densely and finally reticulate-coriaceous. First to sixth tergites entirely or almost entirely densely and distinctly reticulate- or areolate-coriaceous, sculpture becoming finer to distal tergites, posterior margins of third to sixth tergite finely coriaceous to almost smooth. Vertex almost entirely glabrous. Mesoscutum mainly glabrous, with short and sparse white setae narrowly arranged along notauli and laterally. Setae of hind tibia semi-erect, short and sparse, their length on dorsal surface 0.4-0.5 times maximum width of tibia.

CoLour. Body mainly dark reddish brown, head paler. Palpi dark brown, faintly paler apically. Antenna mainly brownish yellow to almost yellow, basally with reddish tint, apical three segments dark brown to black. Legs mainly dark reddish brown, fore and middle coxae yellowish brown; hind tibia anteriorly always same colour as remaining part of tibia; all tarsi mainly light reddish brown. Ovipositor sheath mainly brownish yellow or pale brown, dark brown posteriorly. Fore wing strongly darkened at most part, with contrasting small hyaline or almost white spots and stripes (Fig. 00), with transverse hyaline 
stripe cross narrowest part of submedial cell. Pterostigma mainly dark brown, white in basal 0.4 and in short apical area.

\section{Male}

Unknown.
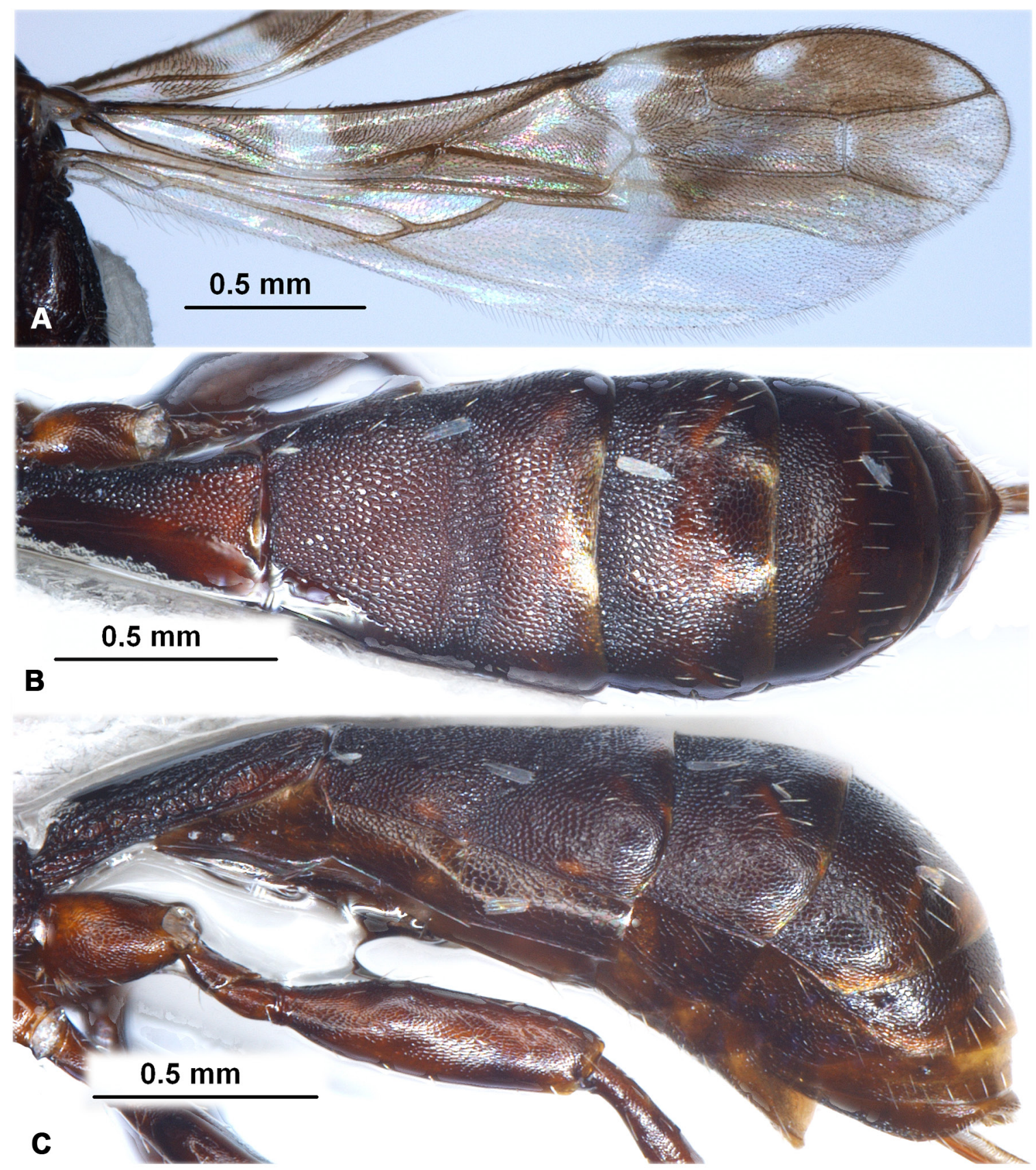

Fig. 42. Platyspathius (P.) longicaudis Belokobylskij sp. nov., holotype, + (RMNH). A. Wings. B. Metasoma, dorsal view. C. Metasoma, lateral view. 


\section{Distribution}

Yemen.

Genus Rhaconotinus Hedqvist, 1965

\section{Type species}

Rhaconotinus caboverdensis Hedqvist, 1965, by original designation.

\section{Remarks}

This genus was restored as a valid by Belokobylskij (2019) and Belokobylskij et al. (2019) on the basis of recently established phylogenetic affinity (Jasso-Martínez et al. 2019). Rhaconotinus currently has two valid subgenera, Hexarhaconotinus Belokobylskij \& Zaldívar-Riverón, 2021 and Rhaconotinus s. str. (Belokobylskij \& Zaldívar-Riverón 2021).

\section{Distribution}

Afrotropical, Oriental and Palaearctic regions.

Rhaconotinus (Rhaconotinus) albosetosus Belokobylskij sp. nov. urn:1sid:zoobank.org:act:58BD9E91-59F6-47DD-B0FB-1150696C7D25

Figs $43-44$

\section{Comparative diagnosis}

This species is similar to Rh. micholitzi (Belokobylskij, 2001) comb. nov. from Papua New Guinea (Belokobylskij 2001b), but differs from latter in having the vertex entirely transversely striate (mainly granulate and only laterally with short striation in $R h$. micholitzi), frons entirely coarsely reticulaterugose (mainly granulate in $R$ h. micholitzi), precoxal sulcus coarsely crenulate (smooth in $R h$. micholitzi), pterostigma pale in basal third (entirely dark brown in $R h$. micholitzi), mediocubital vein (M+CU1) of fore wing distinctly curved in apical half (weakly curved in Rh. micholitzi), nervulus (cu-a) distinctly postfurcal (almost interstitial in Rh. micholitzi), hind tarsus distinctly longer than hind tibia (almost equal to it in Rh. micholitzi), and the first metasomal tergite short (long in Rh. micholitzi).

\section{Etymology}

The name of this species refers to the Latin words 'albus' (white) and 'setosis' (pubescence), after the white body setosity of this species.

\section{Material examined}

\section{Holotype}

YEMEN - O; "Yemen (6117), Al Kowd, 21-28.viii.2001, light trap, A.v. Harten, S. Al Haruri, RMNH'02"; RMNH.

\section{Paratypes}

YEMEN • 1 ㅇ; "Yemen, Al Kowd, vii.2000, light tr., no 4820, A.v. Harten \& S. Al Haruri, RMNH'01"; RMNH • 1 क; "Yemen (4054), Al Kowd, viii.1999, light trap, A.v. Harten \& S. Al Haruri, RMNH'00"; RMNH.

\section{Description}

\section{Female}

MEASUREMEnTs. Body length 5.5-6.9 mm; fore wing length 3.7-4.5 mm. 
HeaD. Head width 1.3-1.5 times its median length, 1.2-1.3 times width of mesoscutum. Head behind eyes very convex anteriorly, than weakly roundly narrowed; transverse diameter of eye 1.2-1.3 times larger than temple length. Ocelli medium-sized, in triangle with base 1.2 times its sides; POL 1.0-1.4 times Od, $0.45-0.60$ times OOL. Eye glabrous, weakly emarginated opposite antennal sockets, 1.2 times as high as broad. Malar space height $0.40-0.45$ times height of eye, 0.75-0.80 times basal width of mandible. Malar suture fine but rather distinct, curved. Face width almost equal to height of eye and 1.2-1.3 times height of face and clypeus combined. Upper margin of clypeus situated almost on lower level of eyes. Hypoclypeal depression round, its width $0.75-0.80$ times distance from edge of depression to eye, $0.40-0.45$ times width of face. Occipital carina not fused with hypostomal carina below being obliterated for rather long distance upper base of mandible; below present several additional transverse carinae. Vertex convex.

AnTENNA. Antenna slender, weakly setiform, 37-42-segmented, about as long as body. Length of scape 1.7-1.9 times its maximum width. First flagellar segment 5.5-6.0 times longer than its apical width, 1.2 times longer than second segment. Penultimate segment 4.5-4.7 times longer than wide, 0.4 times as long as first segment, $0.7-0.8$ times as long as apical segment; latter apically distinctly pointed.

Mesosoma. Length 2.1-2.2 times its height. Pronotum anteriorly weakly convex (dorsal view), posteriorly strongly and widely convex dorsally (lateral view). Pronotal carina rather distinct, widely separated from posterior margin of pronotum, distances from carina to anterior and posterior margins of pronotum subequal. Mesoscutum weakly and roundly elevated above pronotum. Notauli deep, complete and crenulate. Mesoscutum without median longitudinal depression. Prescutellar depression rather short, deep, its posterior sides straight and obliquely directed posterolaterally, depression with distinct median carina, rather densely and distinctly crenulate, about 0.3 times as long as convex scutellum. Precoxal sulcus distinct, densely rugose-crenulate or crenulate, weakly S-shape, long, running along entire lower part of mesopleuron. Prepectal carina rather strong, not widened below, without lobes opposite fore coxae. Metanotum with distinct but not high subpointed tooth (lateral view). Metapleural lobe rather short, narrow and rounded distally.

WINGS. Length of fore wing 3.9-4.0 times its maximum width. Radial (marginal) cell not or only weakly shortened. Metacarpus (1-R1) 1.15-1.20 times longer than pterostigma, almost 7.0 times longer than distance from apex of radial (marginal) cell to apex of wing. Radial vein (r) arising weakly before middle of pterostigma, from basal 0.45 . First radial abscissa ( $\mathrm{r}$ ) forming distinct obtuse angle with second abscissa (3-SR). Second radial abscissa (3-SR) 2.6-2.7 times longer than first abscissa (r), 0.5 times as long as third abscissa (SR1), 1.5 times longer than first radiomedial vein (2-SR). Second radiomedial (submarginal) cell not widened distally, its length 3.0-3.3 times maximum width, 0.85-1.00 times length of the wide brachial (subdiscal) cell. First medial abscissa (1-SR+M) distinctly sinuate. Recurrent vein $(\mathrm{m}-\mathrm{cu})$ weakly postfurcal. Mediocubital vein $(\mathrm{M}+\mathrm{CU} 1)$ rather distinctly curved to anal vein (1-1A) in distal half. Distance from nervulus (cu-a) to basal vein (1-M) 0.6-0.7 times nervulus (cua) length. Brachial (subdiscal) cell rather gently and almost roundly closed almost on level of recurrent vein $(\mathrm{m}-\mathrm{cu})$; posterior bulla on brachial vein (CU1b) present; posterior abscissa of anal vein (2-1A) (behind brachial vein (CU1b)) shortly present. Hind wing not narrowed basally, 5.4-5.5 times longer than wide. First costal abscissa $(\mathrm{C}+\mathrm{SC}+\mathrm{R}) 0.5-0.6$ times as long as second abscissa (1-SC+R). First abscissa of mediocubital vein $(\mathrm{M}+\mathrm{CU}) \quad 0.50-0.55$ times as long as second abscissa (1-M). Recurrent vein $(\mathrm{m}-\mathrm{cu})$ long and unsclerotised.

Legs. Hind femur with distinct wide baso-dorsal protuberance, its length 2.7-2.9 times maximum width. Hind tarsus 1.1-1.2 times longer than hind tibia. Hind tibia distally with five-eight outside spines. Hind basitarsus 0.6 times combined length of second-fifth segments. Second tarsal segment 0.4-0.5 times as 


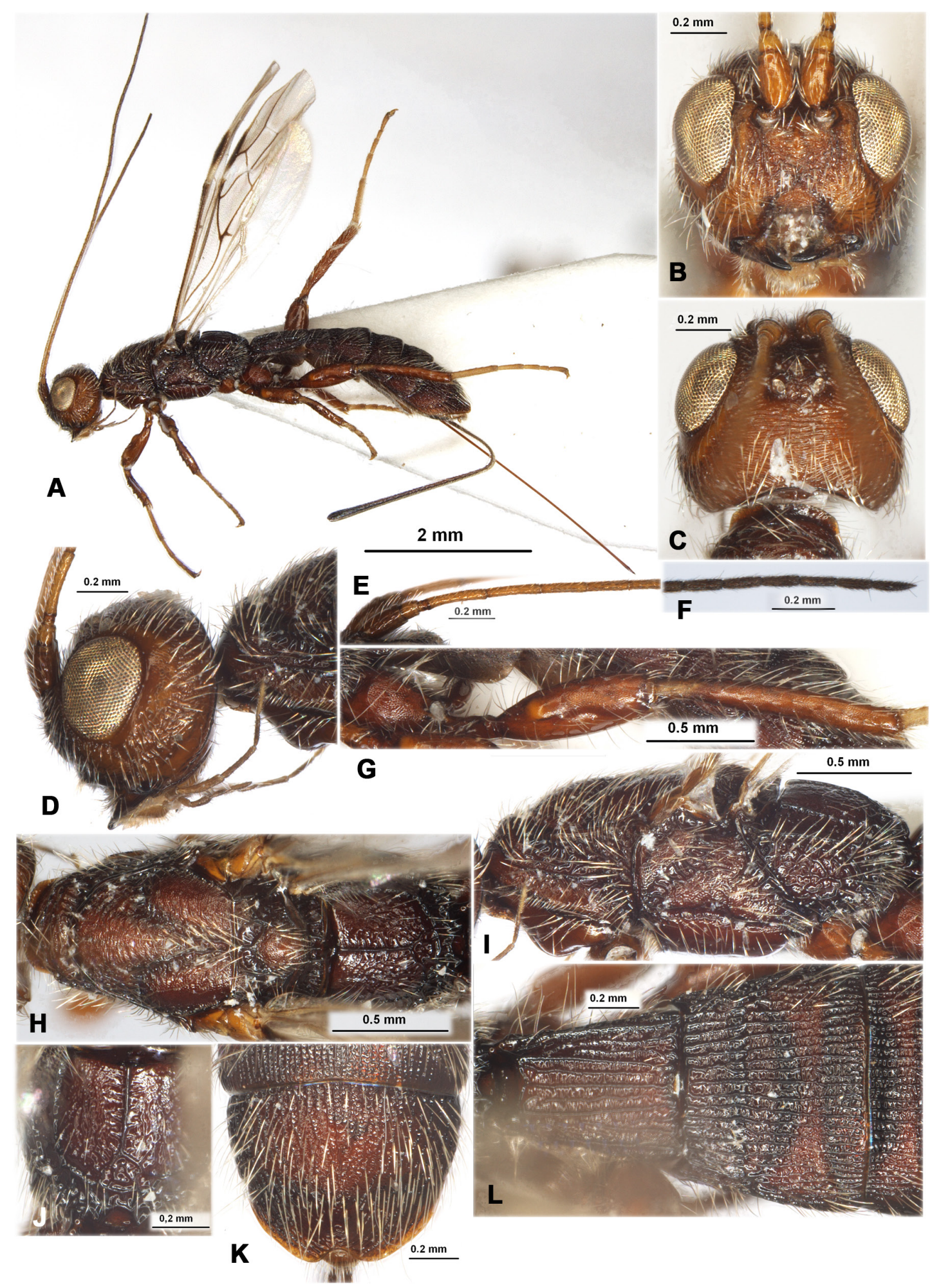

Fig. 43. Rhaconotinus (Rh.) albosetosus Belokobylskij sp. nov., holotype, + (RMNH). A. Habitus, lateral view. B. Head, front view. C. Head, dorsal view. D. Head, lateral view. E. Basal segments of antenna. F. Apical segments of antenna. G. Hind leg. H. Mesosoma, dorsal view. I. Mesosoma, lateral view. J. Propodeum. K. Six metasomal tergite, dorsal view. L. First to third metasomal tergites, dorsal view. 


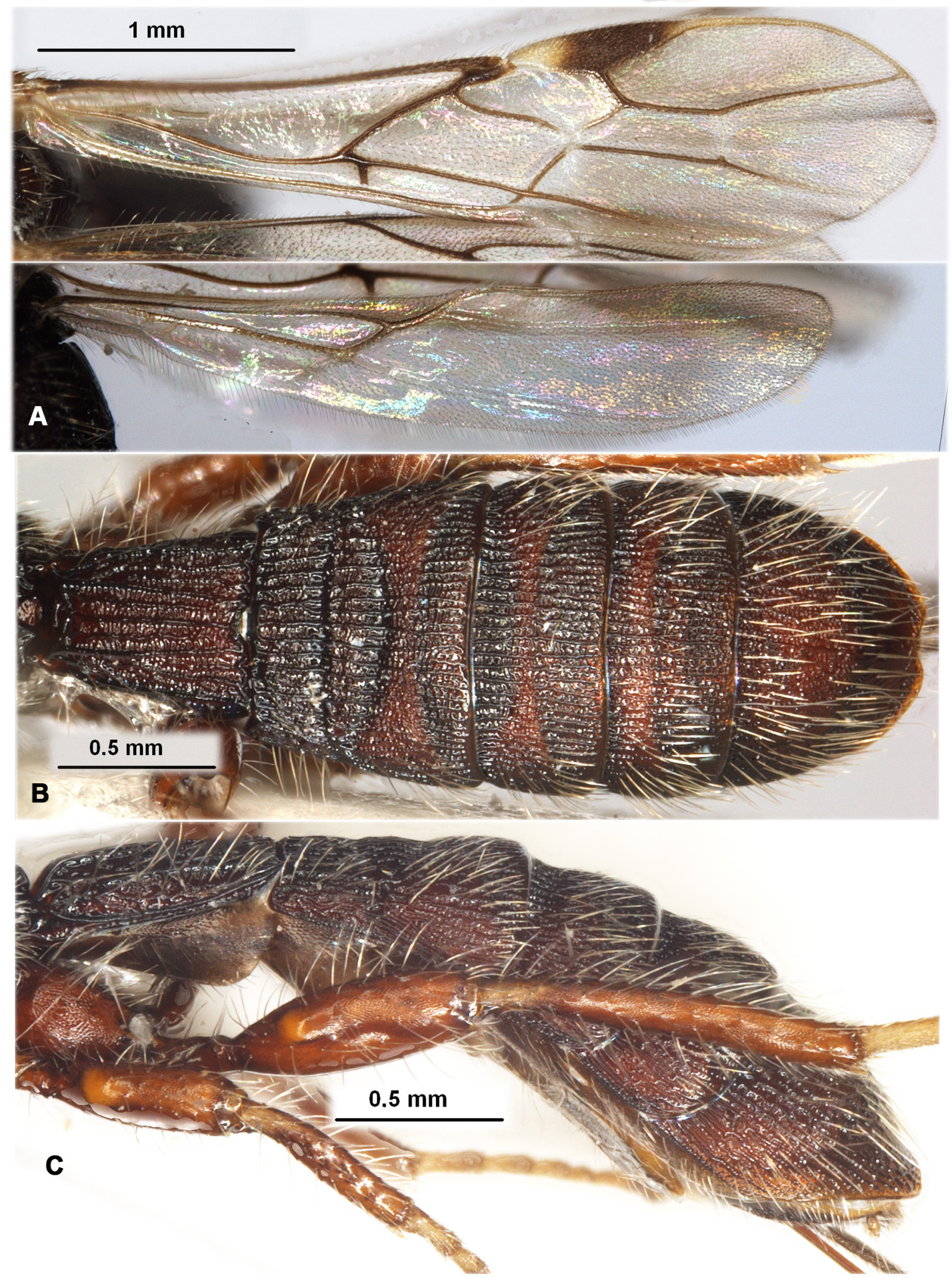

Fig. 44. Rhaconotinus (Rh.) albosetosus Belokobylskij sp. nov., holotype, $q$ (RMNH). A. Wings. B. Metasoma, dorsal view. C. Metasoma, lateral view. 
long as basitarsus, 2.6-3.0 times longer than fourth segment, 1.3-1.4 times longer than fifth segment (without pretarsus).

Metasoma. Metasoma 1.1-1.2 times longer than head and mesosoma combined, with six dorsally visible hard tergites. Posterior width of first tergite 1.8-2.1 times its anterior width; its length 1.05-1.20 times posterior width. Second tergite without anterior area, with distinct, narrow and weakly curved transverse submedian furrow separated lenticular posterior area; this area $0.85-0.90$ times as long as remaining part of tergite. Median length of second tergite (with posterior area) 0.6-0.7 times its anterior width, 1.5-2.0 times length of third tergite. Second suture distinct and rather narrow. Sixth tergite large, rounded in posterior margin, with rather deep and more or less wide median emargination, without posteroventral lobes. Sixth tergite 1.5-2.0 times longer than fifth tergite, 1.7-2.3 times longer than fourth tergite. Ovipositor sheath almost as long as metasoma, 1.5 times longer than mesosoma, 0.8 times as long as fore wing.

SCULPTURE AND PUBESCENCE. Vertex distinctly and densely transversely striate, partly with rugulosity between striae; frons densely and coarsely rugose-reticulate; temple rugose-striate, reticulate-coriaceous anteriorly along eye. Face densely rugulose-reticulate, with curved striation in lower lateral parts. Mesoscutum densely to very densely and finely granulate with rugulosity partly, with distinct striation or rugosity along notauli, with two weakly convergent and undulate striae in medio-posterior third. Scutellum densely reticulate-coriaceous. Mesopleuron entirely densely reticulate-rugulose and partly with rugosity, smooth or almost smooth below precoxal sulcus. Propodeum with basolateral areas delineated by distinct carinae, with median carina in anterior $0.5-0.6$, areola short or not delineated, if present then pentagonal, rugose; basolateral areas densely coriaceous-granulate to partly smooth entirely or at least in anterior half, rugose-reticulate in posterior half, usually distinctly densely striate along carinae. Hind legs densely and finely coriaceous, femur almost smooth in lower half. First tergite with complete dorsal carinae, entirely with several rather sparse longitudinal striae, densely rugulose-striate between striae. Second to fifth tergites distinctly and densely striate, with distinct and dense rugulosity or striation between striae; sixth tergite striate with very dense reticulation in anterior two-thirds, striae more or less concentrically in posterior third. Second-fifth tergites laterally with rather distinct striae and very dense rugosity-reticulation between it. Vertex with long and rather dense semi-erect white setae mainly directed backwards. Mesoscutum with dense semi-erect white setae widely arranged along notauli and anteriorly and in usually single line laterally. Mesopleuron almost entirely with sparse long and white setae. Metasoma dorsally almost entirely with long and rather dense semi-erect white setae directed backwards. Hind tibia dorsally with almost erect or semi-erect, rather dense white setae; length of these setae 1.0-1.3 times maximum width of hind tibia.

CoLour. Head reddish brown or dark reddish brown, faintly paler around eyes; mesosoma and metasoma dark reddish brown to almost black partly. Scape reddish brown, pedicel and flagellum in basal twothirds or half yellowish brown to light reddish brown, flagellum distinctly darkened in apical third or half. Palpi brown to dark brown, sixth maxillary segment light brown or at least paler. All legs reddish brown, fore coxae light reddish brown, all tibiae basally distinctly paler, all tarsi yellowish brown to almost yellow. Ovipositor sheath black. Fore wing faintly and almost evenly infuscate. Pterostigma black to dark brown, whitish yellow or white in basal third or quarter and apically.

\section{Male}

Unknown.

\section{Distribution}

Yemen. 
Rhaconotinus (Rhaconotinus) menippus (Nixon, 1939)

Figs 45-46

Rhaconotus menippus Nixon, 1939: 123.

Rhaconotus decaryi Granger, 1949: 131. syn. nov.

Rhaconotus menippus - Nixon 1941: 501. - Belokobylskij 2001b: 135. - Belokobylskij \& Chen 2004: 355. - Yu et al. 2016.

Rhaconotus decaryi - Yu et al. 2016.

Rhaconotinus menippus - Belokobylskij \& Zaldívar-Riverón 2021: 109.

\section{Material examined}

Lectotype (designated here)

MADAGASCAR • 1 \%; “Madagascar, Berkily, V.1936, A Seyrig coll.”; MNHN.

\section{Additional material}

YEMEN • 1 क; "Yemen (no 2910), Ta'izz, light trap, 5.i-2.ii.1998, A. van Harten, RMNH'98”; RMNH - 6 우; “Yemen (no 2989), Ta'izz, light trap, 1-3.iv.1998, A.v. Harten \& Ahmad Ahwad, RMNH'98"; RMNH • 1 क; same label data as for preceding; ZISP • 3 우; "Yemen (no 3111), Ta'izz, light trap, 26-28.v.1998, A.v. Harten \& Ahmad Ahwad, RMNH'98"; RMNH • 1 \%; "Yemen (3540), Ta'izz,

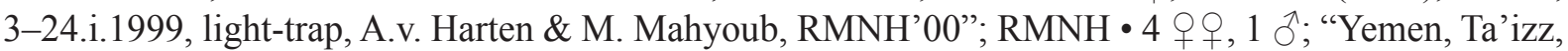
viii.1999, light tr., no 4056, A.v. Harten \& A. Awad, RMNH'01”; RMNH • 1 O ; "Yemen (4057), Ta'izz, ix.1999, light-trap, A.v. Harten \& A. Awad, RMNH'00"; RMNH • 1 क; "Yemen, Ta'izz, xi.1999, light tr., no 4138, A.v. Harten \& A. Awad, RMNH'01"; RMNH • 1 ㅇ; "Yemen (3901), Al Kowd, vii.1999, light-trap, A.v. Harten \& S. Al Haruri. RMNH’00"; RMNH • 2 우; "Yemen (4055), Al Kowd, ix.1999, light-trap, A.v. Harten \& S. Al Haruri, RMNH'00"; RMNH • 118 o $q$; "Yemen, Al Kowd, ii.2000, light tr., no 4431, A.v. Harten \& S. Al Haruri, RMNH'01"; RMNH • 5 우; same label data as for preceding; ZISP・ 19 q o ; "Yemen, Al Kowd, v-vi.2000, light-tr, no 4719, A.v. Harten \& S. Al Haruri, RMNH'01"; RMNH • 1 क; "Yemen, Al Kowd, vii.2000, light tr., no 4820, A.v. Harten \& S. Al Haruri, RMNH'01"; RMNH • 124 क 9 ; "Yemen, Al Kowd, xi.2000, light trap, no 4947/5290, A.v. Harten \& S. Al Haruri. RMNH'01"; RMNH • 5 q ; same label data as for preceding; ZISP • 5 q $q$; "Yemen, Al Kowd, i.2001, light tr., no. 5482, A.v. Harten \& S. Al Haruri, RMNH’01"; RMNH • 4 q ; ; "Yemen, Al Kowd, ii.2001, light tr., no. 5484, A.v. Harten \& S. Al Haruri, RMNH'01"; RMNH • 6 q $q$; "Yemen (5750), Al Kowd, 8-12.vii.2001, light trap, A.v. Harten \& S. Al Haruri. RMNH'02"; RMNH • 2 우; "Yemen (6117), Al Kowd, 21-28.viii.2001, Light trap, A.v. Harten, S. Al Haruri, RMNH'02"; RMNH • 5 o $\circ$; "Yemen (6141), Al Kowd, 16-20.viii.2001, light trap, A.v. Harten \& S. Al Haruri. RMNH'02"; RMNH • 3 q ; "Yemen (8136), Al Kowd, ix.2003, light trap, A.v. Harten, S. Al Haruri, RMNH'03"; RMNH • 1 \%; "Yemen (6090), Al Kadan, x.2001, light trap, A.v. Harten, T. Abdul-Haq. RMNH'02"; RMNH • 3 q ; "Yemen (7189), Al Kadan, v.2002, Light trap, A.v. Harten \& A.R. Al Yarimi. RMNH'02"; RMNH • 2 우; “Yemen (6158), Al Lahima, 17.ix-14.ii.2001, Malaise trap, A.v. Harten, RMNH'02"; RMNH • 1 우 “Yemen (5700), Ar Rujum, 9.iv-5.vi.2001, Mal. trap; A.v. Harten RMNH’02"; RMNH • 11 우; "Yemen (5404), Hammam 'Ali, from coffee-berries (with Ceratitis capitata ?), 14.iii.2001, A.v. Harten, RMNH'02”; RMNH・ 1 \%; same label data as for preceding; ZISP.

\section{Distribution}

Afrotropics: Benin, Madagascar, South Africa, Uganda, *Yemen; Oriental: China, India, Malaysia, Thailand. 


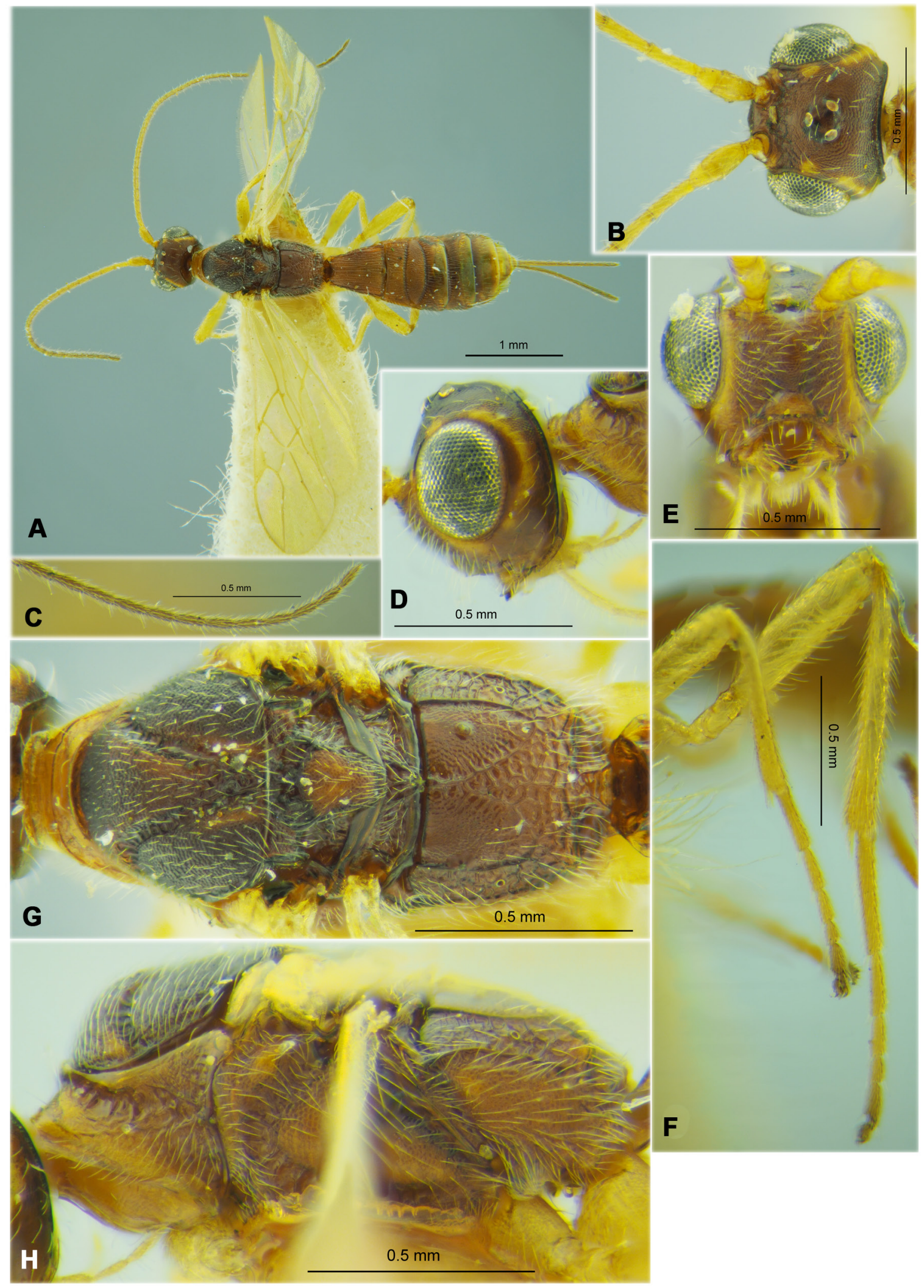

Fig. 45. Rhaconotinus (Rh.) menippus (Nixon, 1939), + (RMNH). A. Habitus, lateral view. B. Head and basal segments of antenna, dorsal view. C. Apical segments of antenna. D. Head, lateral view. E. Head, front view. F. Hind leg. G. Mesosoma, dorsal view. H. Mesosoma, lateral view. 

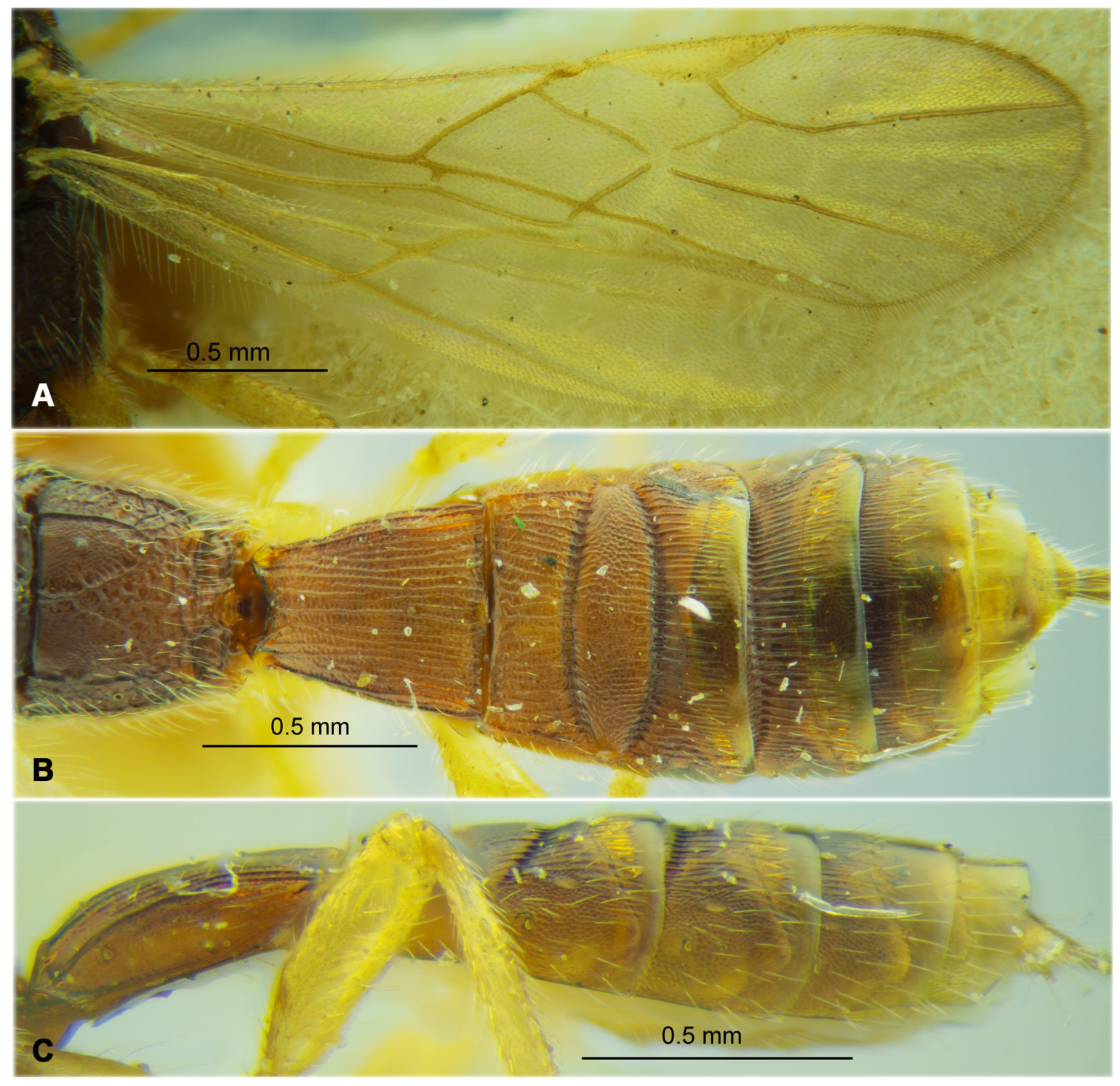

Fig. 46. Rhaconotinus (Rh.) menippus (Nixon, 1939), + (RMNH). A. Wings. B. Propodeum and metasoma, dorsal view. C. Metasoma, lateral view.

\section{Remarks}

Our examination of large amount of material of this species collected in a number of localities in Yemen revealed considerable body colour variation, from a pale reddish brown or yellowish brown colours at least on mesosoma and metasoma, to the complete body almost entirely black.

Genus Rhaconotus Ruthe, 1854

\section{Type species}

Rhaconotus aciculatus Ruthe, 1854, by monotypy.

\section{Remarks}

This is a large and worldwide distributed rhaconotine genus, currently consisting of two subgenera, the monotypic Afrotropical Pararhacon Belokobylskij, 2005, and the widely distributed Rhaconotus s. str. 


\section{Distribution}

Afrotropical, Australasian, Nearctic, Neotropical, Oriental and Palaearctic regions.

\section{Key to Arabian species of the genera Rhaconotus and Rhaconotinus (female)}

1. Metasoma of female with six dorsally visible hardly sclerotised tergites (Figs 44B-C, 46B-C) (Rhaconotinus Hedqvist, 1965)

- Metasoma of female with only five dorsally visible hardly sclerotised tergites (Figs 48B-C, 50B-C, 52B-C, 54D, 56B-C, 58C-D, 60B-C, 62C-D) (Rhaconotus Ruthe, 1854) ................................. 3

2. Transverse diameter of eye 1.2-1.3 times larger than temple length (Fig. 43C). Vertex entirely transversely striate (Fig. 43C). Mesosoma covered by long yellow setae (Fig. 43H-I). Pterostigma mainly dark brown, but pale in basal third (Fig. 44A). Mediocubital vein of fore wing distinctly curved in apical half (Fig. 44A). Sixth tergite in posterior margin with distinct median emargination (Fig. 43K); long, 1.5-2.0 times longer than fifth tergite (Fig. 44B). Ovipositor sheath almost as long as metasoma, 0.8 times as long as fore wing (Fig. 43A). Body length 5.5-6.9 mm

Rhaconotinus (Rh.) albosetosus Belokobylskij sp. nov.

- Transverse diameter of eye 1.8-2.3 times length of temple (Fig. 45B). Vertex entirely granulate and with reticulation at least partly, always without striae (Fig. 45B). Mesosoma covered by short whitish setae (Fig. 45G-H). Pterostigma entirely yellow or pale brown (Fig. 46A). Mediocubital vein of fore wing weakly curved in apical half (Fig. 46A). Sixth tergite in posterior margin without median emargination; short, usually not or only sometimes weakly longer than fifth tergite (Fig. 46B). Ovipositor sheath distinctly shorter than metasoma, 0.4-0.5 times as long as fore wing (Fig. 45A). Body length 3.6-5.0 mm Rhaconotinus (Rh.) menippus (Nixon, 1939)

3. Second metasomal suture without additional transverse furrow and without posterior area (Figs $54 \mathrm{C}$, $58 \mathrm{C}, 60 \mathrm{~B}, 62 \mathrm{C})$

- Second metasomal suture with additional transverse furrow and with delineated posterior area (Figs 48C, 50B, 52B, 56B)

4. Fifth metasomal tergites with two long processes on its posterior margin (Figs 61J-K, 62C-D). Body length $3.3 \mathrm{~mm}$ Rhaconotus (Rh.) vanharteni Belokobylskij sp. nov.

- Fifth metasomal tergites without processes on its posterior margin, evenly curved (Figs 54B, 58C, 60B)

5. Pterostigma mainly dark brown and only its basal third yellow (Fig. 60A). Body length 3.0$5.4 \mathrm{~mm}$ Rhaconotus (Rh.) sudanensis Wilkinson, 1927

- Pterostigma entirely yellow or pale brown (Figs 54A, 58A)

6. Vertex with dense and rather long setae directed laterally (Fig. 57B). Mesosoma entirely or almost entirely with dense and rather long pale setae (Fig. 57G-H). Body length 4.2-5.5 mm

Rhaconotus (Rh.) scirpophagae Wilkinson, 1927

- Vertex with dense and rather long setae directed laterally (Fig. 53B). Mesosoma entirely or almost entirely with dense and rather long pale setae (Fig. 57F-G). Body length 3.0-4.2 mm

Rhaconotus (Rh.) manolus Nixon, 1941

7. Radial (marginal) cell of fore wing strongly shortened; metacarpus (1-R1) $0.5-0.6$ times as long as pterostigma, about 0.7 times as long as distance from apex of radial cell to apex of wing (Fig. 50A). Body length $3.6-4.8 \mathrm{~mm}$ .Rhaconotus (Rh.) brevicellularis Belokobylskij sp. nov. 
- Radial (marginal cell) of fore wing not or only weakly shortened, proceeding till apex of wing; metacarpus (1-R1) 0.9-1.1 times as long as pterostigma, much longer than distance from apex of radial cell to apex of wing (Figs 48A, 52A, 56A)

8. Third and fourth metasomal tergite in distal third or quarter with deep and coarsely crenulate transverse furrow (Figs 47H-I, 48C). Second metasomal suture with distinct median break (Figs 47H, 48C). Fifth metasomal tergite at least medially widely smooth or almost smooth (Figs 47I, 48C). Legs mainly reddish brown to dark reddish brown (Fig. 47A). Body length 2.4-3.8 mm

Rhaconotus (Rh.) arabicus Belokobylskij, 2001

- Third and fourth metasomal tergite in distal third without transverse crenulate furrows (Figs 52B, 56B). Second metasomal suture without median break (Figs 52B, 55J, 56B). Fifth metasomal tergite entirely coarsely striate (Figs 52B, 55K, 56B). Legs mainly yellow, but sometimes with brownish tint or brownish yellow, often dark basally (Figs 51A, 55A)

9. Fifth tergite on posterior margin without median incision (Fig. 52B). Temple longer, transverse diameter of its eye 1.4-1.7 times temple length (Fig. 51B). Upper margin of clypeus situated on or weakly below than lower level of eyes (Fig. 51C). First metasomal tergite longer, 1.2-1.4 times longer than its posterior width (Fig. 52B). Second metasomal tergite 2.6-3.3 times longer than third tergite (Fig. 52B). Ovipositor sheath shorter, 0.8-0.9. times longer than mesosoma, 0.4 times as long as fore wing (Fig. 51A). Body length 2.6-3.8 mm

Rhaconotus (Rh.) magniareolus Belokobylskij sp. nov.

- Fifth tergite on posterior margin with small median incision (Fig. 55K). Temple shorter, transverse diameter of its eye 2.2-2.5 times temple length (Fig. 55B). Upper margin of clypeus situated distinctly upper than lower level of eyes (Fig. 55C). First metasomal tergite longer, 1.5-1.6 times longer than its posterior width (Figs 55J, 56B). Second metasomal tergite 1.4-1.6 times longer than third tergite (Figs 55J, 56B). Ovipositor sheath longer, 1.1-1.2 times longer than mesosoma, 0.50-0.55 times as long as fore wing (Fig. 55A). Body length 3.5-4.2 mm

Rhaconotus (Rh.) microexcavatus Belokobylskij sp. nov.

Rhaconotus (Rhaconotus) arabicus Belokobylskij, 2001

Figs $47-48$

Rhaconotus arabicus Belokobylskij, 2001a: 463.

Rhaconotus arabicus - Yu et al. 2016. — Edmardash et al. 2020: 43.

\section{Material examined}

UNITED ARAB EMIRATES • 1 q; "United Arab Emirates, Wadi Safad (5026), at light, 1-8.vii.2006, $25^{\circ} 1^{\prime} \mathrm{N}, 56^{\circ} 1^{\prime}$ E, A.v. Harten, RMNH'06”; RMNH 1 \%; same label data as for preceding; ZISP • 2 우 ; “United Arab Emirates, Wadi Safad (5020), at light, 14-21.v.2006, $25^{\circ} 1^{\prime}$ N, 56 $6^{\circ} 1^{\prime}$ E, A.v. Harten, RMNH'06"; RMNH • 1 q; "United Arab Emirates, Wadi Safad (5029), at light, 17-24.vi.2006, 251' N, $56^{\circ} 1^{\prime}$ E, A.v. Harten, RNMH'06”; RMNH -2 우; “United Arab Emirates, Wadi Safad (5867-5902),

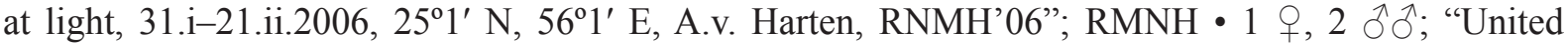
Arab Emirates, Sharjah Desert park (2517), light trap, 20.x-8.xi.2005, $25^{\circ} 17^{\prime}$ N, 55 $42^{\prime}$ ' E, A.v. Harten, RNMH'06"; RMNH・1 Oें; same label data as for preceding; ZISP • 1 q; "United Arab Emirates, Sharjah

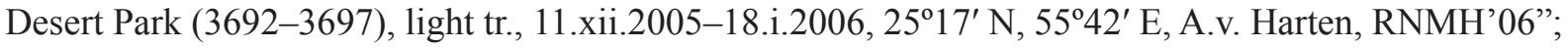

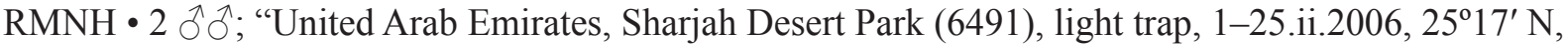

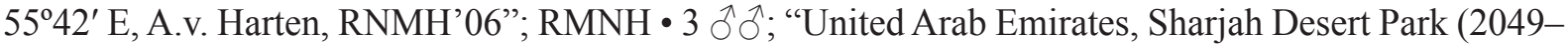

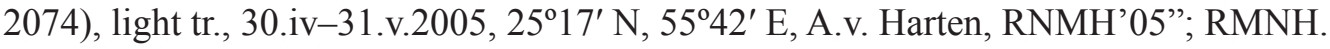




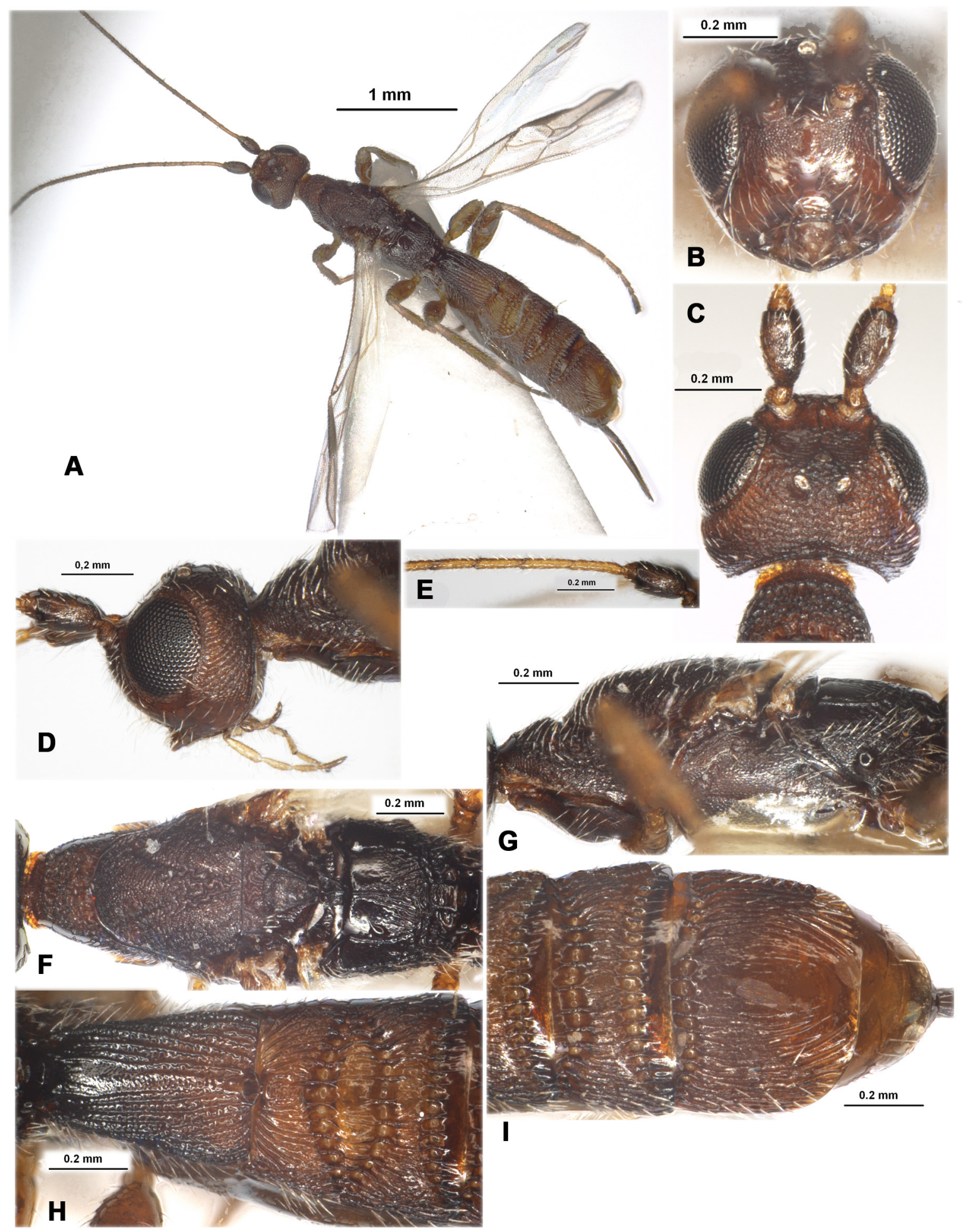

Fig. 47. Rhaconotus (Rh.) arabicus Belokobylskij, 2001, $q$ (RMNH). A. Habitus, dorsal view. B. Head, front view. C. Head, dorsal view. D. Head, lateral view. E. Basal segments of antenna. F. Mesosoma, dorsal view. G. Mesosoma, lateral view. H. First to third metasomal tergites, dorsal view. I. Fourth and fifth metasomal tergites, dorsal view. 
YEMEN • 1 q; "Yemen (6117), Al Kowd, 21-28.viii.2001, Light trap, A.v. Harten, S. Al Haruri, RMNH'02"; RMNH • 1 क; "Yemen (8136), Al Kowd, ix.2003, light trap, A.v. Harten, S. Al Haruri, RMNH'03"; RMNH • 1 đ'; "Yemen (4054), Al Kowd, viii.1999, light-trap, A.v. Harten \& S. Al Haruri, RMNH'00"; RMNH • 1 万े; "Yemen, Al Kowd, v-vi.2000, light tr., no 4719, A.v. Harten \& S, Al Haruri,

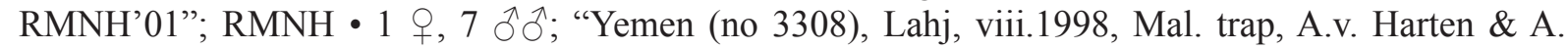

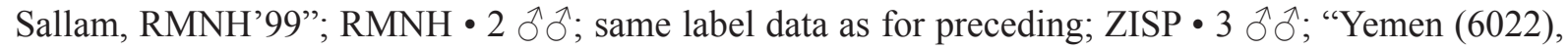
Lahj, vii-ix.2001, Mal. trap, A.v. Harten \& A. Sallam, RMNH'02"; RMNH.

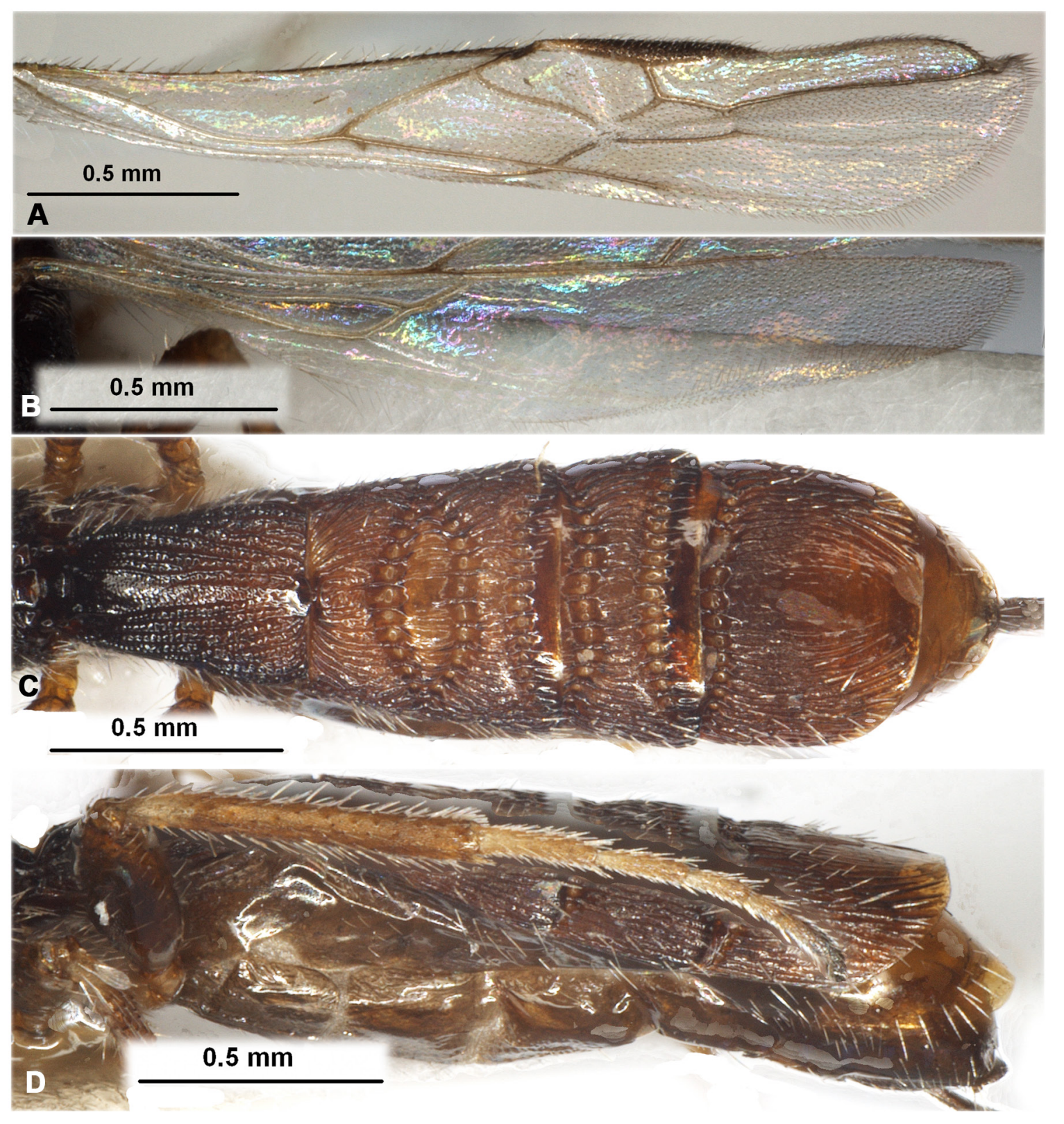

Fig. 48. Rhaconotus (Rh.) arabicus Belokobylskij, 2001, q (RMNH). A. Fore wing. B. Hind wing. C. Metasoma, dorsal view. D. Metasoma, lateral view. 


\section{Description}

Male (first record)

Body length 1.6-3.1 mm; fore wing length 1.2-2.1 mm. Antenna with 18-27-segmented. Fore wing 4.34.8 times longer than maximum width. Hind femur 2.5-2.8 times longer than maximum broad. Metasoma 1.1-1.2 times longer than head and mesosoma combined, with sixth hardly sclerotised and visible tergites. First metasomal tergite 1.3-1.5 times longer than posterior width. Median length of posterior area of second tergite $0.8-1.0$ times median length of the remaining part of tergite. Median length of second tergite (with posterior area) 0.8-1.0 times its anterior width, 1.6-2.0 times length of third tergite. Sometimes third tergite with shallow and rather weak transverse distal furrow and fourth tergite almost without such furrow. Fifth tergite sometimes (in small specimens) almost smooth on posterior half and weakly reticulate-rugulose in anterior half; on posterior margin almost straight, 1.0-1.3 times as long as practically smooth to very finely reticulate sixth tergite, 1.1 times longer than fourth tergite. Otherwise similar to female.

\section{Distribution}

Saudi Arabia, *UAE, *Yemen.

Rhaconotus (Rhaconotus) brevicellularis Belokobylskij sp. nov. urn:1sid:zoobank.org:act:95C2B19B-AD03-4EA3-B93A-75D8627B70A4

Figs 49-50

\section{Comparative diagnosis}

This new species is very similar to Rhaconotus arabicus Belokobylskij, 2001, from the Arabian Peninsula (Belokobylskij 2001a), but differs from the latter by having the radial (marginal) and second radiomedial (submarginal) cells strongly shortened (both long in Rh. arabicus), metasoma densely and entirely sculptured (sparsely sculptured and with smooth areas in Rh. arabicus), propodeum without basolateral areas (with such delineated arears in Rh. arabicus), first flagellar segment of antenna slender, 5.6 times longer than its posterior width (thick, about 4.0 longer than its posterior width in Rh. arabicus), ovipositor longer (shorter in Rh. arabicus).

Rhaconotus brevicellularis sp. nov. resembles by the strongly shortened radial (marginal) cell of fore wing, the Central Asian Rh. acmaeoderellae Belokobylskij, 1990 (Belokobylskij 1990), described from Uzbekistan, but it distinctly differs in having the posterior area on the second metasomal tergite long and anteriorly distinctly delineated by a deep and narrow furrow (short posterior area delineated by a shallow and wide furrow in $R h$. acmaeoderellae), presence of deep and coarsely crenulate curved transverse furrows on posterior third of the third and fourth tergites (absence of such furrows in Rh. acmaeoderellae), metasomal tergites long (short in Rh. acmaeoderellae), and fore wing faintly and evenly infuscate (distinctly maculate in Rh. acmaeoderellae).

\section{Etymology}

Named after its strongly shortened radial (marginal) cell of the fore wing.

\section{Material examined}

Holotype

UNITED ARAB EMIRATES • +; "United Arab Emirates, Wadi Safad (5020), at light, 14-21.v.2006, $25^{\circ} 1^{\prime} \mathrm{N}, 56^{\circ} 1^{\prime}$ E, A.v. Harten, RNMH'06”; RMNH.

\section{Paratypes}

UNITED ARAB EMIRATES • 1 क; "United Arab Emirates, Wadi Safad (5029), at light, 17-24.vi.2006, $25^{\circ} 1^{\prime}$ N, 56 $6^{\circ}$ ' E, A.v. Harten, RNMH'06”; ZISP • 1 क; “United Arab Emirates, Sharjah Desert Park 


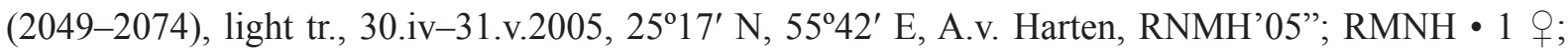
"United Arab Emirates, Wadi Maidaq (4003), white \& yellow pan tr., 14-25.i.2006, 251' N, 56 ${ }^{\circ} 0^{\prime}$ E, A.v. Harten, RNMH'06”; RMNH • 1 O; “United Arab Emirates, Wadi Wurayah (1368), Mal. \& water

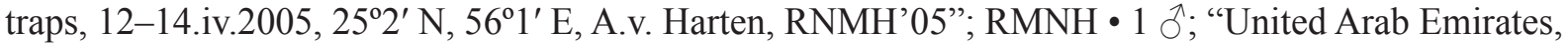

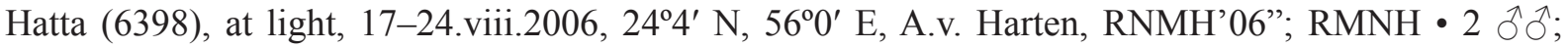
"United Arab Emirates, Sharhah x Khor Kalba (6308-6311), light trap, 31.v-7.vi.2006, A.v. Harten, RNMH'06"; RMNH.

\section{Description}

Female

Measurements. Body length 3.6-4.8 mm; fore wing length 2.4-2.9 mm.

HEAD. Head width 1.4-1.5 times its median length, 1.2-1.3 times width of mesoscutum. Head behind eyes distinctly evenly convex-roundly narrowed; transverse diameter of eye 1.6-1.8 times larger than temple length. Ocelli medium-sized, in triangle with base 1.2-1.3 times its sides; POL 1.1-1.5 times Od, 0.5-0.6 times OOL. Eye with short and very sparse setae, very weakly emarginated opposite antennal sockets, 1.2 times as high as broad. Malar space height $0.45-0.50$ times height of eye, 1.0-1.1 times basal width of mandible. Malar suture absent. Face width about 1.1 times height of eye and 1.2-1.3 times height of face and clypeus combined. Upper margin of clypeus situated weakly upper than lower level of eyes. Hypoclypeal depression subround, its width 0.7-0.8 times distance from edge of depression to eye, $0.35-0.40$ times width of face. Occipital carina not fused with hypostomal carina below being obliterated for rather long distance upper base of mandible. Vertex convex.

Antenna. Antenna long, slender, setiform, 29-35-segmented, about as long as body. Length of scape 1.7-1.8 times its maximum width. First flagellar segment 5.6-6.4 times longer than its apical width, 1.0-1.1 times as long as second segment. Penultimate segment 3.6-4.5 times longer than wide, 0.5 times as long as first segment, $0.8-0.9$ times as long as apical segment; latter acuminate apically.

Mesosoma. Length 2.2-2.3 times its height. Pronotum anteriorly almost straight (dorsal view), distinctly convex dorsally in posterior half (lateral view). Pronotal carina almost indistinct or fine. Mesoscutum not highly and gently-roundly elevated above pronotum. Notauli rather deep, wide, complete and crenulate. Mesoscutum without median longitudinal depression. Prescutellar depression rather long, shallow, its posterior side almost straight laterally or weakly curved, without median carina, densely and distinctly rugulose-granulate, $0.35-0.45$ times as long as convex scutellum. Precoxal sulcus distinct, deep medially and shallow anteriorly and posteriorly, crenulate-reticulate, almost straight, long, running along entire lower part of mesopleuron. Prepectal carina distinct, not widened below, without lobes opposite fore coxae. Metanotum with short, obtuse wide tubercle (lateral view). Metapleural lobe rather long, narrow, rounded distally.

WINGS. Length of fore wing 3.8-4.0 times its maximum width. Radial (marginal) cell strongly shortened, 3.6-3.8 times longer than maximum width. Metacarpus (1-R1) 0.5-0.6 times as long as pterostigma, about 0.7 times as long as distance from apex of radial (marginal) cell to apex of wing. Radial vein (r) arising from middle of pterostigma. First radial abscissa (r) forming obtuse angle with second abscissa (3-SR). Second radial abscissa (3-SR) 1.4-1.7 times longer than first abscissa (r), 0.3 times as long as curved third abscissa (SR1), 0.65-0.80 times as long as first radiomedial vein (2-SR). Second radiomedial (submarginal) cell short, not widened distally, its length 1.7-2.0 times maximum width, 0.6-0.8 times as long as narrow brachial (subdiscal) cell. First medial abscissa (1-SR+M) distinctly sinuate. Recurrent vein $(\mathrm{m}-\mathrm{cu})$ postfurcal. Mediocubital vein $(\mathrm{M}+\mathrm{CU} 1)$ distinctly curved to anal vein $(1-1 \mathrm{~A})$ in distal half. Distance from nervulus (cu-a) to basal vein (1-M) 0.7-1.2 times nervulus (cu-a) length. Brachial (subdiscal) cell gently and roundly closed almost on level of recurrent vein (m-cu); posterior bulla 


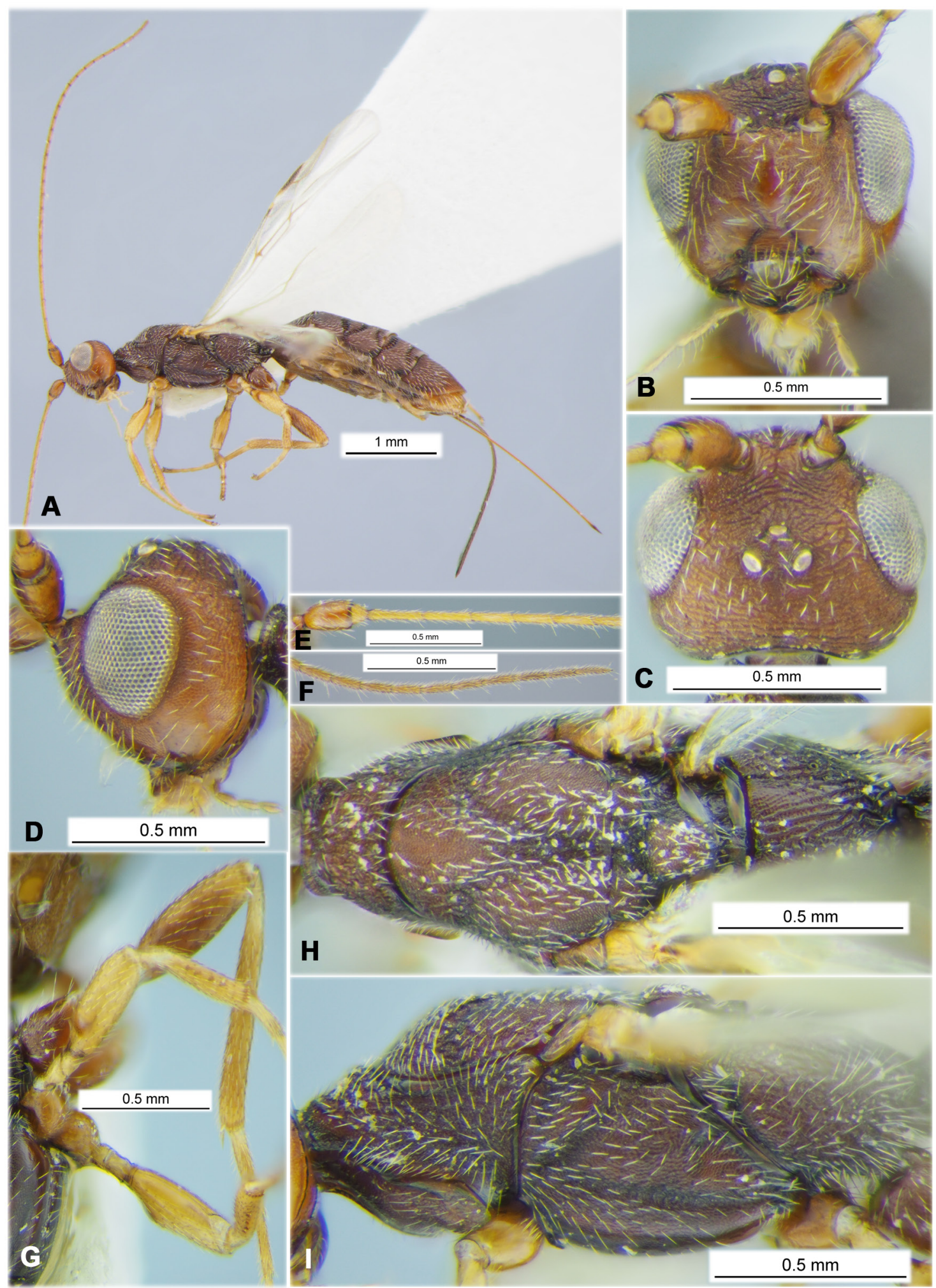

Fig. 49. Rhaconotus (Rh.) brevicellularis Belokobylskij sp. nov., holotype, $q$ (RMNH). A. Habitus, lateral view. B. Head, front view. C. Head, dorsal view. D. Head, lateral view. E. Basal segments of antenna. F. Apical segments of antenna. G. Hind leg. H. Mesosoma, dorsal view. I. Mesosoma, lateral view. 
on brachial vein (m-cu) present; posterior abscissa of anal vein (2-1A) (behind brachial vein (CU1b)) absent. Hind wing not narrowed basally, 4.8-5.0 times longer than wide. First costal abscissa $(\mathrm{C}+\mathrm{SC}+\mathrm{R})$ $0.7-0.8$ times as long as second abscissa (1-SC+R). First abscissa of mediocubital vein (M+CU) $0.4-0.5$ times as long as second abscissa (1-M). Recurrent vein (m-cu) rather long, unsclerotised, distinctly evenly curved to base of wing.

LEGS. Hind femur with rather distinct basodorsal protuberance, its length 2.8-3.2 times maximum width. Hind tarsus as long as hind tibia. Hind tibia posteriorly with four-five outside spines. Hind basitarsus $0.5-0.6$ times combined length of second-fifth segments. Second tarsal segment $0.60-0.65$ times as long as basitarsus, 1.3-1.5 times longer than fifth segment (without pretarsus).

Metasoma. Metasoma 1.2-1.3 times longer than head and mesosoma combined, with five dorsally visible hard tergites. Maximum subposterior width of first tergite 2.4-2.7 times its anterior width; its length 1.15-1.20 times posterior width. Second tergite without basal area, with long posterior area separated anteriorly by deep curved crenulate transverse furrow. Median length of posterior area 0.7-0.8 times median length of the remaining part of second tergite. Median length of second tergite (with posterior area) 0.8-0.9 times its anterior width, 1.8-2.0 times length of third tergite. Second suture deep and twice curved. Third and fourth tergites with deep curved and densely crenulate transverse furrow on posterior half or third; fourth tergite with similar basal transverse furrow. Fifth tergite enlarged, on posterior margin distinctly curved, without processes, tubercles or incisure, without tubercles laterally. Fifth tergite 1.5-1.7 times longer than fourth tergite. Ovipositor sheath $0.70-0.85$ times as long as metasoma, 1.2-1.4 times longer than mesosoma, 0.7 times as long as fore wing.

SCUlPTURE AND PUBeSCENCE. Vertex entirely densely curvedly transversely striate with very dense reticulation between striae and sometimes fine granulation between striae; frons distinct sinuate striate and densely reticulate-rugose; temple coarsely striate and densely reticulate-coriaceous in upper half, finely coriaceous to almost smooth in lower half. Face mainly densely reticulate-granulate in upper twothird, almost smooth to smooth medially and sometimes narrowly in lower parts. Mesoscutum entirely densely granulate, with reticulation along notauli, with two sinuate striae with rugosity in narrow medio-posterior third. Scutellum densely granulate. Mesopleuron entirely densely reticulate-coriaceous, finely coriaceous below precoxal sulcus. Propodeum without delineated areas, with several convergent posteriorly longitudinal carinae and dense rugulosity between striae; areola absent, but present here rugose area in medioposterior third. Hind coxa rugulose-coriaceous in upper half and finely coriaceous in lower half; hind femur mainly densely reticulate-coriaceous. First tergite without distinctly dorsal carinae. First to fifth tergites distinctly, densely and at least partly curvedly or divergently striate, with distinct and very dense rugulosity between striae; striae on posterior half of fifth tergite subparallel. Second-fifth tergites laterally with fine and dense striae and fine rugosity between it. Vertex with short and rather dense semi-erect white setae directed forwards. Mesoscutum almost entirely with rather dense and semi-erect white setae, its lateral lobes on narrow submedial parts and medial lobe narrow medially glabrous. Metasoma dorsally almost entirely with short and dense semi-erect white setae directed backwards. Hind tibia dorsally with semi-erect and rather dense short white setae, length of these setae $0.4-0.6$ times maximum width of hind tibia.

Colour. Head light reddish brown to yellowish brown, reddish brown dorsally; mesosoma and metasoma dark reddish brown or reddish brown, metasoma below mainly pale brown. Scape of antenna light reddish brown, pedicel and basal four-fifth flagellum yellowish brown or light reddish brown, remaining apical part of flagellum brownish. Palpi brownish yellow to yellow. Fore and middle legs yellowish brown, coxa infuscate; hind coxa and femur reddish brown, hind tibia and tarsus yellow; all tibiae basally paler, fifth tarsal segments distally infuscate. Ovipositor sheath dark brown to black. Fore 
wing subhyaline, faintly infuscate along veins. Pterostigma dark brown, yellow to pale brown in basal third and shortly apically.

\section{Male}

Body length 3.0-4.3 mm; fore wing length 1.9-2.7 mm. Antenna 25-31-segmented. Length of scape 1.4-1.5 times its maximum width. Hind femur thicker, its length 2.8 times maximum width. Metasoma
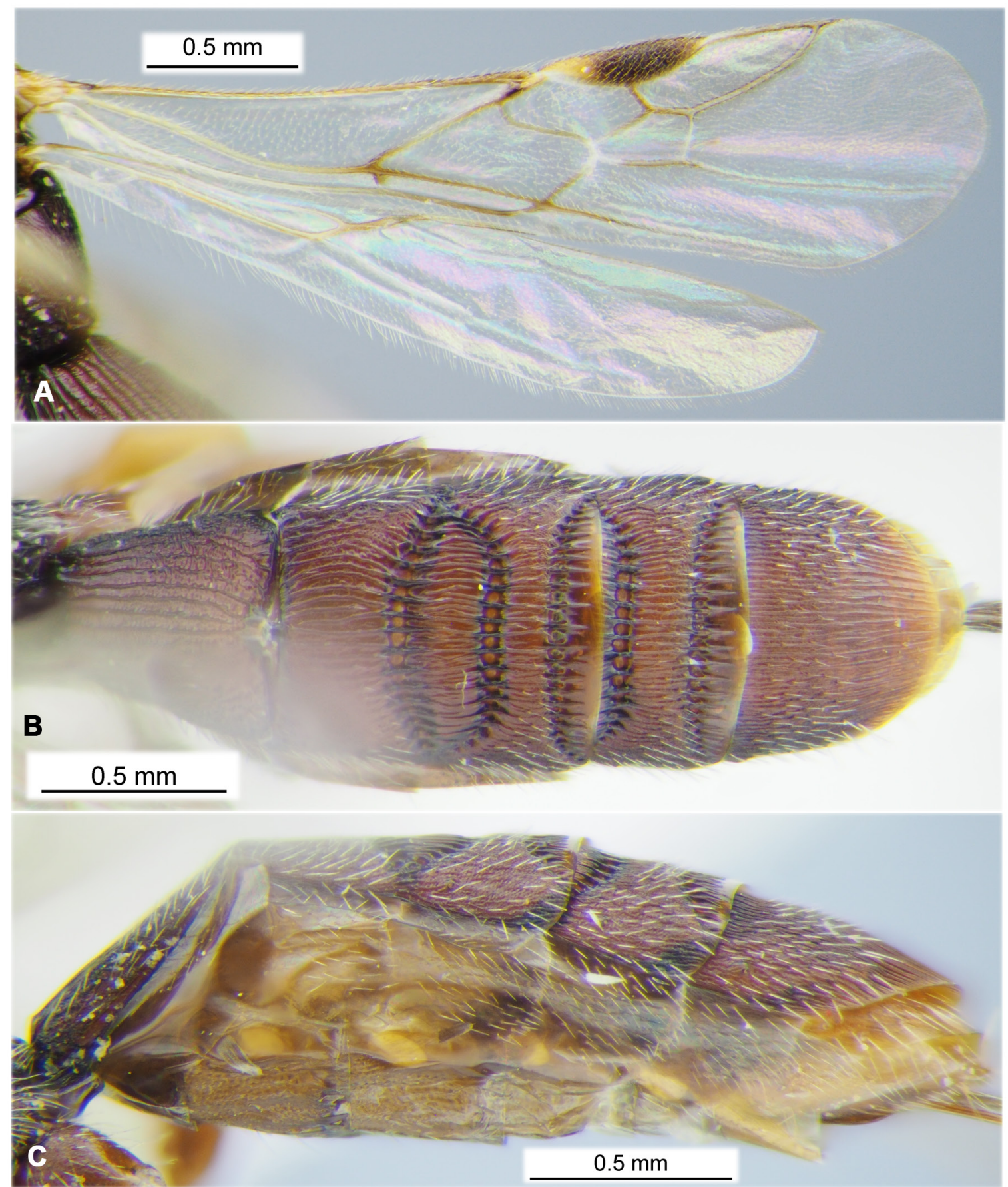

Fig. 50. Rhaconotus (Rh.) brevicellularis Belokobylskij sp. nov., holotype, + (RMNH). A. Wings. B. Metasoma, dorsal view. C. Metasoma, lateral view. 
narrow, with sixth tergite only shortly covered by previous (fifth) tergite. Length of first tergite 1.20 1.35 times its posterior width. Median length of second tergite (with posterior area) equal to its anterior width. Fifth tergite 1.2-1.5 times longer than fourth tergite,1.6-2.0 times longer than sixth tergites. Otherwise similar to female.

\section{Distribution}

UAE.

Rhaconotus (Rhaconotus) magniareolus Belokobylskij sp. nov. urn:1sid:zoobank.org:act:FF51F51C-7589-41E5-9D5E-FAF3BD514C02

Figs $51-52$

\section{Comparative diagnosis}

This species is very similar to Rhaconotus testaceiceps (Cameron, 1910) from Indonesia (Belokobylskij 2001b), but differs from the latter by having the posterior lenticular area of the second metasomal tergite medially long (short in Rh. testaceiceps), first metasomal tergite narrow and long (wide and short in in $R h$. testaceiceps), brachial (second subdiscal) cell distally closed distinctly before recurrent vein (m-cu) (almost on the vein level in Rh. testaceiceps), propodeum with unclear delineated basolateral areas (with distinctly delineated areas in $R h$. testaceiceps).

Rhaconotus magniareolus sp. nov. is also similar to Rh. arabicus, but differs from it in having the third and fourth metasomal tergites in the distal third without transverse furrows (with a deep and coarsely crenulate transverse curved furrow in Rh. arabicus), second metasomal suture without a median break (with median break in Rh. arabicus), fifth metasomal tergite entirely coarsely striate (at least medially widely smooth or almost smooth in $R h$. arabicus); legs mainly yellow (mainly reddish brown to dark reddish brown in Rh. arabicus); second radiomedial (submarginal) cell longer (short in Rh. arabicus).

\section{Etymology}

This new species is named after the strongly enlarged posterior area of the second metasomal tergite.

\section{Material examined}

\section{Holotype}

YEMEN • O; "Yemen (4054), Al Kowd, viii.1999, light-trap, A.v. Harten \& S. Al Haruri, RMNH'00"; RMNH.

\section{Paratypes}

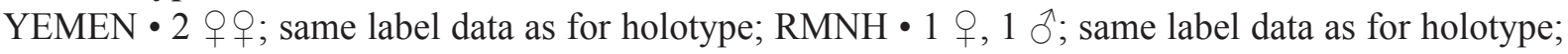
ZISP • 1 o; "Yemen (4055), Al Kowd, ix.1999, light trap, A.v. Harten \& S. Al Haruri. RMNH'00"; RMNH • 3 q 9 ; "Yemen (3901), Al Kowd, vii.1999, light-trap, A.v. Harten \& S. Al Haruri. RMNH'00"; RMNH - 1 क; "Yemen, Al Kowd, xi.2000, light trap, no 4947/5290, A.v. Harten \& S. Al Haruri. RMNH'01"; RMNH • 1 q; same label data as for preceding; ZISP • 2 q + ; "Yemen, Al Kowd, vii.2000, light tr., no 4820, A.v. Harten \& S. Al Haruri, RMNH'01"; RMNH • 4 우, 1 §’; "Yemen, Al Kowd, v-vi.2000, light tr., No 4719, A.v. Harten \& S, Al Haruri, RMNH'01"; RMNH • 1 q; same label data as for preceding; ZISP • 1 q; "Yemen, Al Kowd, i.2001, light tr., no 5482, A.v. Harten \& S. Al Haruri. RMNH'02"; RMNH • 1 क; "Yemen (5750), Al Kowd, 8-12.vii.2001, light trap, A.v. Harten \& S. Al Haruri. RMNH'02"; RMNH • 1 क; "Yemen (6141), Al Kowd, 16-20.viii.2001, light trap, A.v. Harten \& S. Al Haruri. RMNH'02"; RMNH • 2 o o ; "Yemen (8136), Al Kowd, ix.2003, light trap, A.v. Harten, S. Al Haruri, RMNH'03"; RMNH • 1 \%; "Yemen (5404), Hammam 'Ali, from coffee-berries (with Ceratitis capitata ?), 14.iii.2001, A.v. Harten, RMNH'02"; RMNH • 1 \%; "Yemen (no 2910), Ta'izz, 

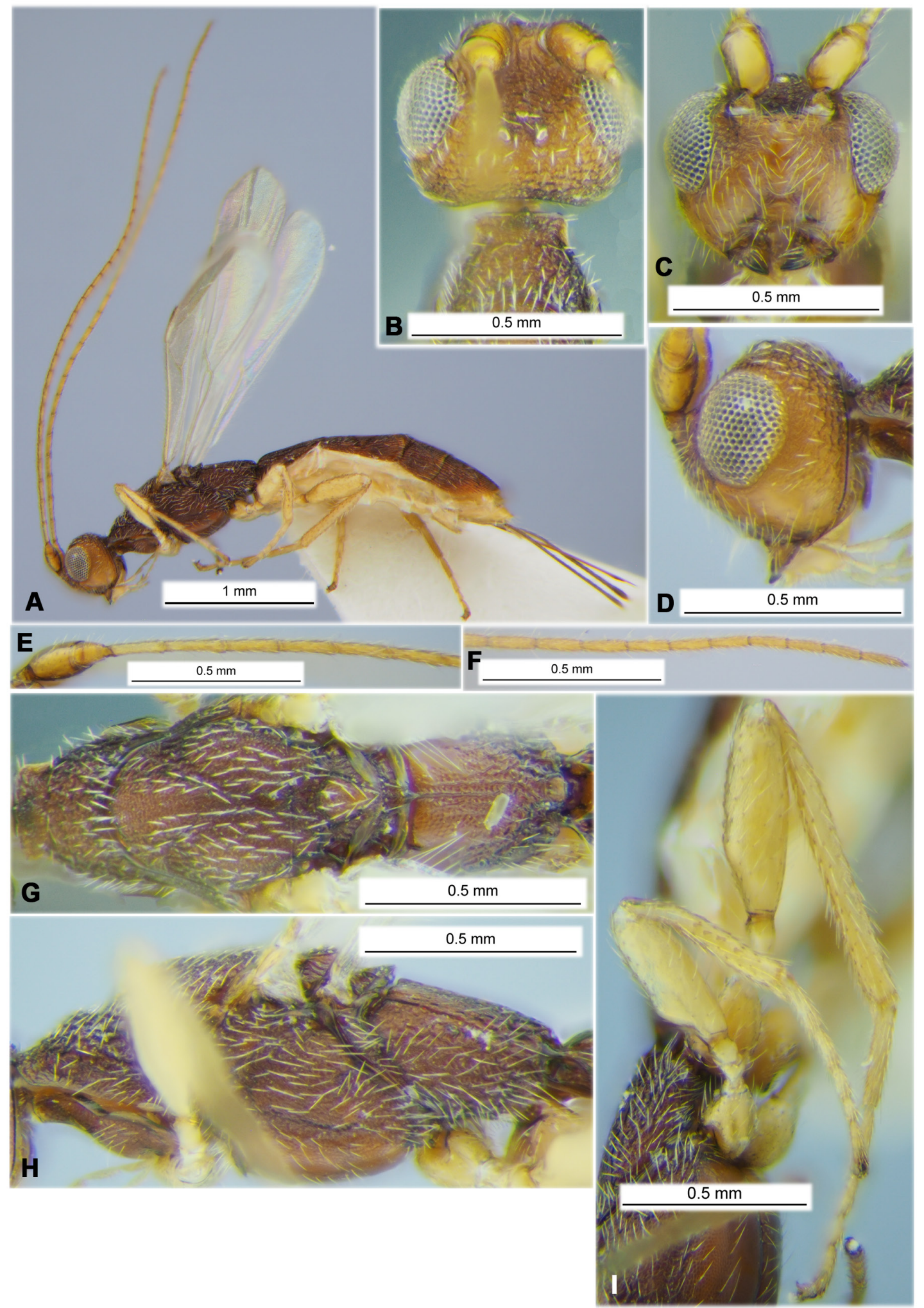

Fig. 51. Rhaconotus (Rh.) magniareolus Belokobylskij sp. nov., holotype, $q$ (RMNH). A. Habitus, lateral view. B. Head, dorsal view. C. Head, front view. D. Head, lateral view. E. Basal segments of antenna. F. Apical segments of antenna. G. Mesosoma, dorsal view. H. Mesosoma, lateral view. I. Hind leg. 
light trap, 5.i-2.ii.1998, A.v. Harten, RMNH'98”; RMNH • 1 q; "Yemen (no 2989), Ta'izz, light trap, 1-3.iv.1998, A.v. Harten \& Ahmad Ahwad, RMNH'98"; RMNH • 1 ö; "Yemen, Ta'izz, viii.1999, light tr., no 4056, A.v. Harten \& A. Awad, RMNH'01”; RMNH.

\section{Description}

\section{Female}

MEASUREMENTs. Body length 2.6-3.8 mm; fore wing length 1.9-2.4 mm.

HEAD. Head width 1.4-1.5 times its median length, 1.3-1.4 times width of mesoscutum. Head behind eyes distinctly evenly convex-roundly narrowed; transverse diameter of eye 1.4-1.7 times larger than temple length. Ocelli small, arranged in triangle with base 1.1-1.2 times its sides; POL 1.2-1.7 times Od, 0.4-0.6 times OOL. Eye glabrous, weakly emarginated opposite antennal sockets, $1.2-1.3$ times as high as broad. Malar space height $0.5-0.6$ times height of eye, almost equal to basal width of mandible. Malar suture absent. Face width 1.1-1.2 times height of eye and 1.15-1.20 times height of face and clypeus combined. Upper margin of clypeus situated on or weakly below than lower level of eyes. Hypoclypeal depression round, its width 0.6-0.7 times distance from edge of depression to eye, $0.3-0.4$ times width of face. Occipital carina not fused with hypostomal carina below being obliterated for short or very short distance upper base of mandible but connected with it by additional rugae. Vertex weakly convex.

Antenna. Antenna long, slender, weakly setiform, 26-33-segmented, about 1.2 times longer than body. Length of scape 1.5-1.7 times its maximum width. First flagellar segment 4.3-4.8 times longer than its apical width, 0.9-1.0 times as long as second segment. Penultimate segment 3.5-3.7 times longer than wide, about 0.7 times as long as first segment, $0.85-0.90$ times as long as apical segment; latter acuminate apically.

Mesosoma. Length 2.1-2.3 times its height. Pronotum anteriorly almost straight (dorsal view), distinctly convex dorsally in posterior half (lateral view). Pronotal carina rather distinct and situated medially. Mesoscutum not highly and gently-roundly elevated above pronotum. Notauli rather deep, wide, complete and sparse crenulate with dense granulation. Mesoscutum without median longitudinal depression. Prescutellar depression rather long, shallow anteriorly and deeper posteriorly, its posterior side laterally straight and oblique, without or with weak median carina, densely and distinctly rugulose and sometimes with additional undulate striae, 0.4 times as long as convex scutellum. Precoxal sulcus distinct, rather deep, crenulate-reticulate, weakly curved, long, running along almost whole length of lower part of mesopleuron. Prepectal carina distinct, weakly widened below, without lobes opposite fore coxae. Metanotum with short or distinct, distally acuminate and basally wide dorsal process (lateral view). Metapleural lobe distinct, rather narrow, rounded distally.

WINGS. Length of fore wing 4.2-4.4 times its maximum width. Radial (marginal) cell not shortened, 4.0-4.5 times longer than maximum width. Metacarpus (1-R1) 1.0-1.1 times as long as pterostigma. Radial vein (r) arising almost from middle of pterostigma. First radial abscissa (r) forming obtuse angle with second abscissa (3-SR). Second radial abscissa (3-SR) 2.8-3.0 times longer than first abscissa (r), 0.45-0.50 times as long as straight third abscissa (SR1), 1.1-1.4 times longer than first radiomedial vein (2-SR). Second radiomedial (submarginal) cell median-sized, not widened distally, its length 2.6-2.9 times maximum width, 1.2-1.4 times longer than rather narrow brachial (subdiscal) cell. First medial abscissa $(1-\mathrm{SR}+\mathrm{M})$ sinuate. Recurrent vein $(\mathrm{m}-\mathrm{cu})$ postfurcal. Mediocubital vein $(\mathrm{M}+\mathrm{CU} 1)$ rather distinctly curved to anal vein (1-1A) in distal half. Distance from nervulus (cu-a) to basal vein (1-M) 1.1-1.5 times nervulus (cu-a) length. Brachial (subdiscal) cell gently and roundly closed rather distinctly before level of recurrent vein (m-cu); posterior bulla on brachial vein (CU1b) absent; posterior abscissa of anal vein (2-1A) (behind brachial vein (CU1b)) absent. Hind wing not narrowed basally, 5.2-5.8 
times longer than wide. First costal abscissa $(\mathrm{C}+\mathrm{SC}+\mathrm{R})$ 0.5-0.6 times as long as second abscissa (1$\mathrm{SC}+\mathrm{R})$. First abscissa of mediocubital vein $(\mathrm{M}+\mathrm{CU}) 0.35-0.40$ times as long as second abscissa (1-M). Recurrent vein (m-cu) short, unsclerotized, antefurcal, oblique, straight.

LEGS. Hind femur with rather distinct basodorsal protuberance, its length 3.2-3.4 times maximum width. Hind tarsus $0.9-1.0$ times as long as hind tibia. Hind tibia posteriorly with four outside spines. Hind basitarsus about 0.5 times combined length of second-fifth segments. Second tarsal segment $0.55-0.60$ times as long as basitarsus, 1.2-1.3 times longer than fifth segment (without pretarsus).
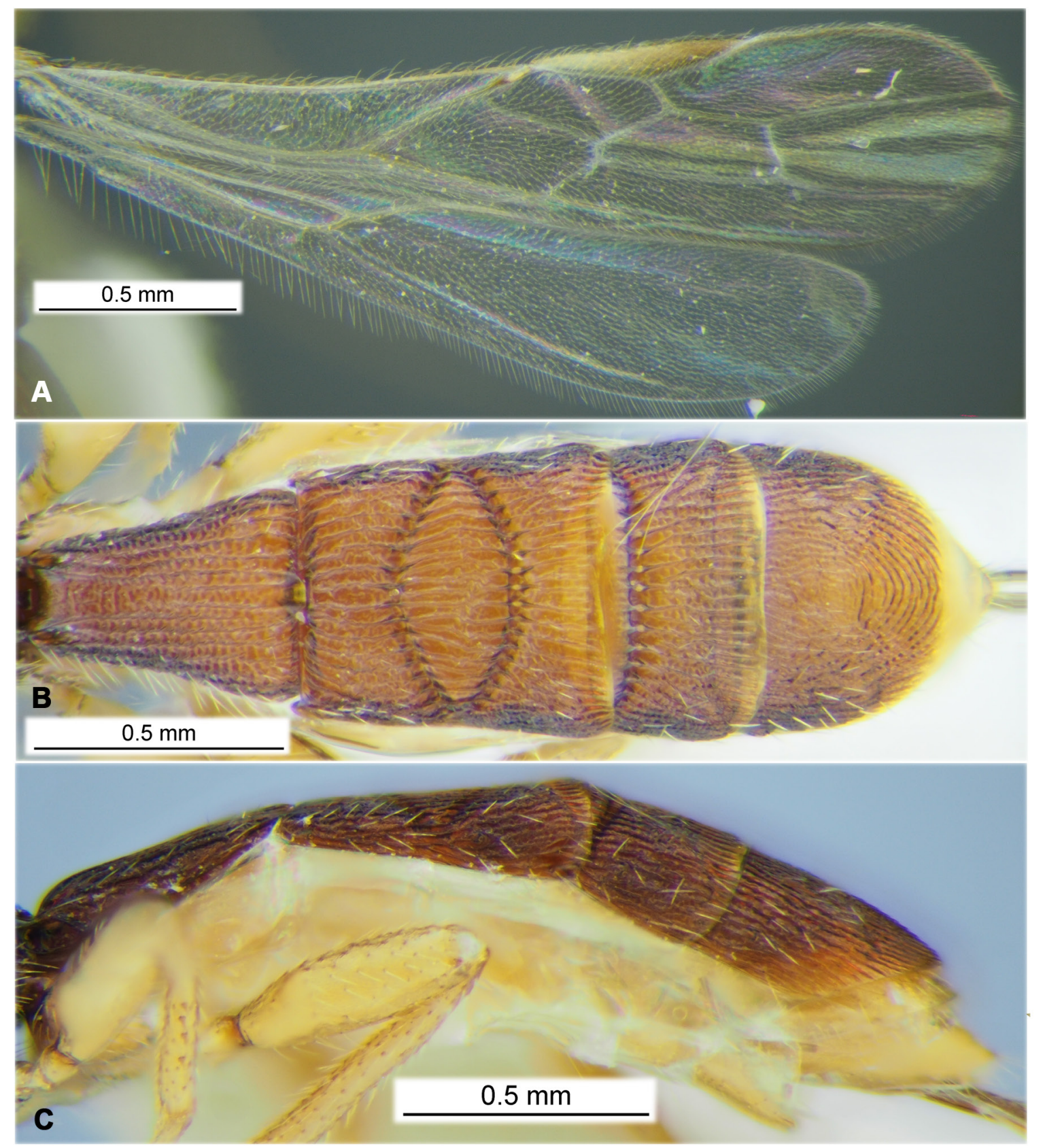

Fig. 52. Rhaconotus (Rh.) magniareolus Belokobylskij sp. nov., holotype, $q$ (RMNH). A. Wings. B. Metasoma, dorsal view. C. Metasoma, lateral view. 
Metasoma. Metasoma 1.2-1.3 times longer than head and mesosoma combined, with five dorsally visible hardly sclerotised tergites. Maximum subposterior width of first tergite 2.2-2.6 times its anterior width; its length 1.2-1.4 times posterior width. Second tergite without anterior area, with long posterior area separated anteriorly by deep and evenly curved crenulate transverse furrow. Median length of posterior area 1.3-1.4 times median length of remaining part of second tergite. Median length of second tergite (with posterior area) 0.9 times its anterior width, 2.6-3.3 times length of third tergite. Second suture deep and evenly curved. Third and fourth tergites without posterior transverse furrow; fourth tergite with crenulate deep anterior transverse furrow. Fifth tergite enlarged, on posterior margin distinctly curved, without processes, tubercles or incisure, without tubercles laterally. Fifth tergite 1.7-1.8 times longer than fourth tergite. Ovipositor sheath $0.5-0.6$ times as long as metasoma, 0.8-0.9 times longer than mesosoma, 0.4 times as long as fore wing.

SCULPTURE AND PUBESCENCE. Vertex entirely densely undulately-curvedly transversely striate with dense reticulation between striae; frons distinctly reticulate-striate; temple mainly subvertical or oblique undulately striate with reticulation, finely coriaceous to smooth in lower quarter to third. Face densely reticulate-granulate or reticulate-rugulose in upper half or two-thirds, almost smooth to smooth in rather narrow medial vertical area and laterally in lower half to third. Mesoscutum densely granulate-reticulate situated in dense transverse curved lines, with rugosity along notauli, with rugosity in narrow area in medioposterior third. Scutellum densely and rather weakly granulate-reticulate. Mesopleuron mainly densely reticulate-coriaceous, finely and densely granulate to coriaceous below precoxal sulcus. Propodeum without delineated areas, with weakly and sometimes incompletely delineated long basolateral areas; areola absent; basolateral areas sometimes smooth or almost smooth anteriorly, mainly or sometimes entirely rugose-reticulate with fine striation; remaining part of propodeum rugose-striate or rugose-reticulate. Hind coxa densely rugulose-areolate dorsally, reticulate-coriaceous laterally, almost smooth ventrally; hind femur mainly reticulate-coriaceous, almost smooth below. First tergite with distinctly and weakly narrowed complete dorsal carinae. First to fifth tergites coarsely, densely and at least partly curvedly or divergently striate, with distinct and dense rugulosity between striae; striae on posterior half of fifth tergite distinctly curved and without rugosity between striae. Second-fifth tergites laterally with distinct dense striae and rugosity between it. Vertex with short and rather sparse semi-erect white setae directed forwards. Mesoscutum mainly with rather sparse and semi-erect white setae, its medial lobe on medial part glabrous. Metasoma dorsally partly with short and sparse semi-erect white setae. Hind tibia dorsally with semi-erect and rather dense short pale setae, length of these setae 0.6-0.8 times maximum width of hind tibia.

CoLour. Head mainly yellowish brown, reddish brown dorsally; mesosoma and metasoma dark reddish brown or reddish brown, but sometimes dark reddish brown, metasoma below mainly pale brown or almost yellow. Scape and pedicel of antenna light reddish brown to reddish brown, most basal part of flagellum yellow to yellowish brown, remaining short apical part of flagellum sometimes becoming brownish. Palpi yellow. Legs mainly yellow, hind coxa distinctly infuscate. Ovipositor sheath brown. Fore wing faintly infuscate. Pterostigma brownish yellow in basal half, brown in apical half.

\section{Male}

Body length 2.3-2.7 mm; fore wing length 1.6-2.1 mm. Antenna 23-26-segmented; scape wide, its length 1.4 times maximum width. Metasoma 1.1-1.4 times longer than head and mesosoma combined, with six dorsally visible sclerotised tergites; sixth tergite finely reticulate-rugulose and almost smooth in posterior fifth. Median length of second tergite (with posterior area) 1.1 times its anterior width; median length of its posterior area 0.9-1.0 times median length of remaining part of tergite. Fifth tergite on posterior margin almost straight, 1.0-1.2 times as long as sixth tergite, 1.1-1.2 times longer than fourth tergite. Face widely smooth, rugose-reticulate in upper sublateral third.

\section{Distribution}

Yemen. 
BELOKOBYLSKIJ S.A. \& VAN ACHTERBERG C., Review of Doryctinae from UAE and Yemen

Rhaconotus (Rhaconotus) manolus Nixon, 1941

Figs 53-54

Rhaconotus manolus Nixon, 1941: 483.

Rhaconotus manolus - Yu et al. 2016.

\section{Material examined}

UNITED ARAB EMIRATES - 2 qo+; "United Arab Emirates, Sharjah Desert Park (186), light tr.,

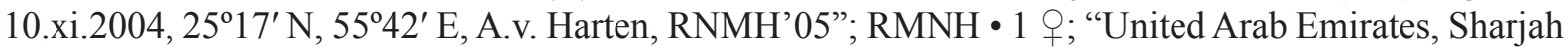

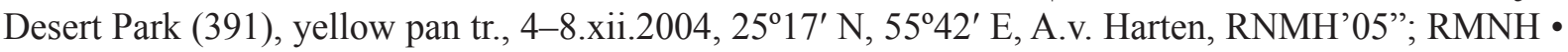

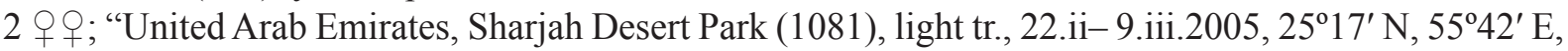
A.v. Harten, RNMH'05”; RMNH - 3 q o⿱; "United Arab Emirates, Sharjah Desert Park (1308), light

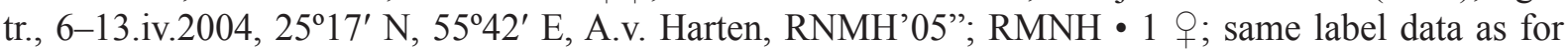
preceding; ZISP • 3 क 9 ; "United Arab Emirates, Sharjah Desert Park (1394), light tr., 13-23.iv.2004, $25^{\circ} 17^{\prime}$ N, $55^{\circ} 42^{\prime}$ E, A.v. Harten, RNMH'05”; RMNH 2 2 9 ; “ “United Arab Emirates, Sharjah Desert Park

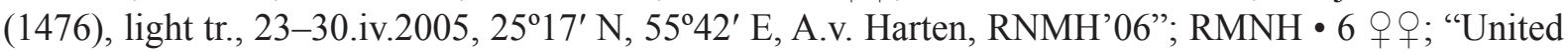
Arab Emirates, Sharjah Desert Park (1556), light tr., 30.iv-7.v.2005, $25^{\circ} 17^{\prime}$ N, 55 $42^{\prime}$ E, A.v. Harten, RNMH'06"; RMNH • 1 q; same label data as for preceding; ZISP • 7 q $q, 1$ đ; " "United Arab Emirates,

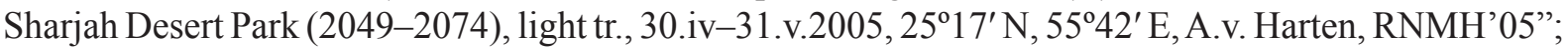
RMNH • 1 \%; same label data as for preceding; ZISP • 2 9 ; "United Arab Emirates, Sharjah Desert

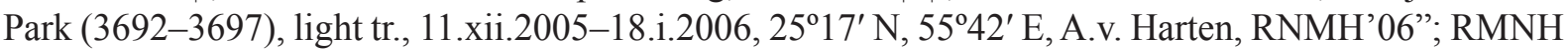

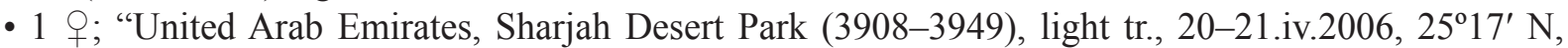

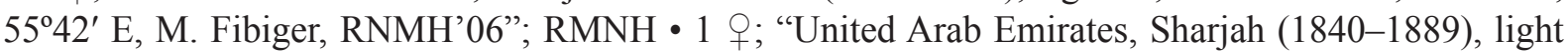

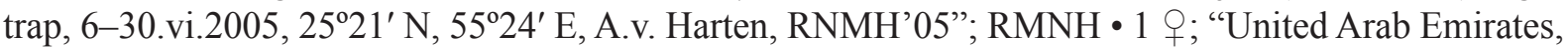
Fujairah (1314), light tr., 13-19.iv.2005, $25^{\circ} 0^{\prime} \mathrm{N}, 56^{\circ} 2^{\prime}$ E. A.v. Harten, RMNH'05"; RMNH • 1 q; “United Arab Emirates, Fujairah (1587), hand coll., 2-13.v.2005, $25^{\circ} 0^{\prime}$ N, 56 $2^{\prime}$ E. A.v. Harten, RMNH'05”; RMNH • 1 q; "United Arab Emirates, NARC near Sweihan (1410), light trap, 1.ii-14.iii.2005, 242' N, 552' E, A.v. Harten, RNMH'05”; RMNH - 1 क; "United Arab Emirates, Wadi Safad (5020), at light,

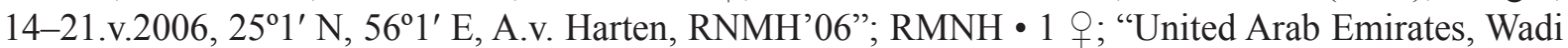

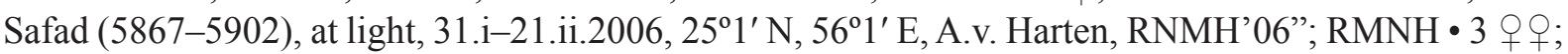

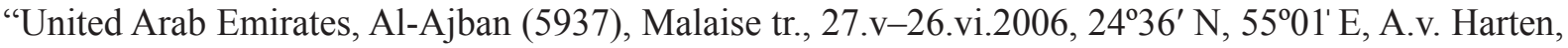
RNMH'06"; RMNH • 1 \%; same label data as for preceding; ZISP • 2 + $\circ$; "United Arab Emirates, Al-

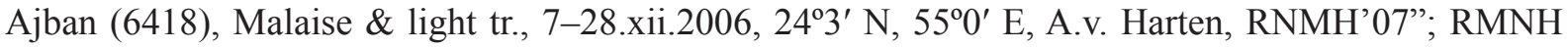

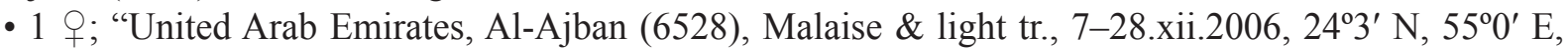
A.v. Harten, RNMH'06"; RMNH 1 1 +, 1 ơ; "United Arab Emirates, SSW of ad-Dhaid (6154), light tr.,

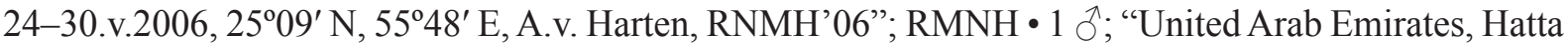

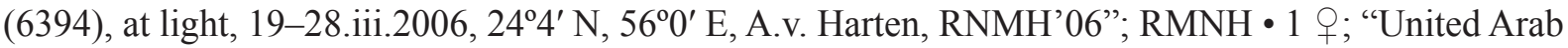

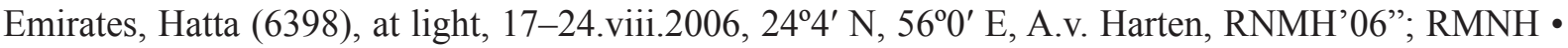
1 q; “United Arab Emirates, Wadi Maidaq (6470), Malaise trap, 29.vii-26.viii.2006, 251' N, 56 $6^{\circ}$ ' E, A.v. Harten, RNMH'07”; RMNH.

YEMEN • 1 o ; "Yemen (no 2893), Al Kadan (20 km N Bajil), Mal. trap, 3.xii.1997-16.ii.1998, A.v. Harten, RMNH'98"; RMNH • 1 o'; "Yemen (no 3308), Lahj, viii.1998, Mal. trap, A.v. Harten \& A. Sallam, RMNH'99"; RMNH • 1 क; "Yemen (6814), Lahj, iii-v.2002, Mal. trap, A.v. Harten \& A. Sallam, RMNH'02"; RMNH • 1 q, 1 ठ̊;; "Yemen (3901), Al Kowd, vii.1999, light-trap, A.v. Harten \& S. Al Haruri. RMNH'00"; RMNH • 1 क; "Yemen (4054), Al Kowd, viii.1999, light-trap, A.v. Harten \& S. Al Haruri. RMNH'00"; RMNH • 1 ô;; "Yemen (4055), Al Kowd, ix.1999, light trap, A.v. Harten \& S. Al Haruri. RMNH'00"; ZISP • 1 q; "Yemen, Al Kowd, ii.2000, light tr., no 4431, A.v. Harten \& S. Al Haruri, RMNH’01”; RMNH • 2 o $\circ$; “Yemen, Al Kowd, v-vi.2000, light tr., no. 4719, A.v. Harten \& 


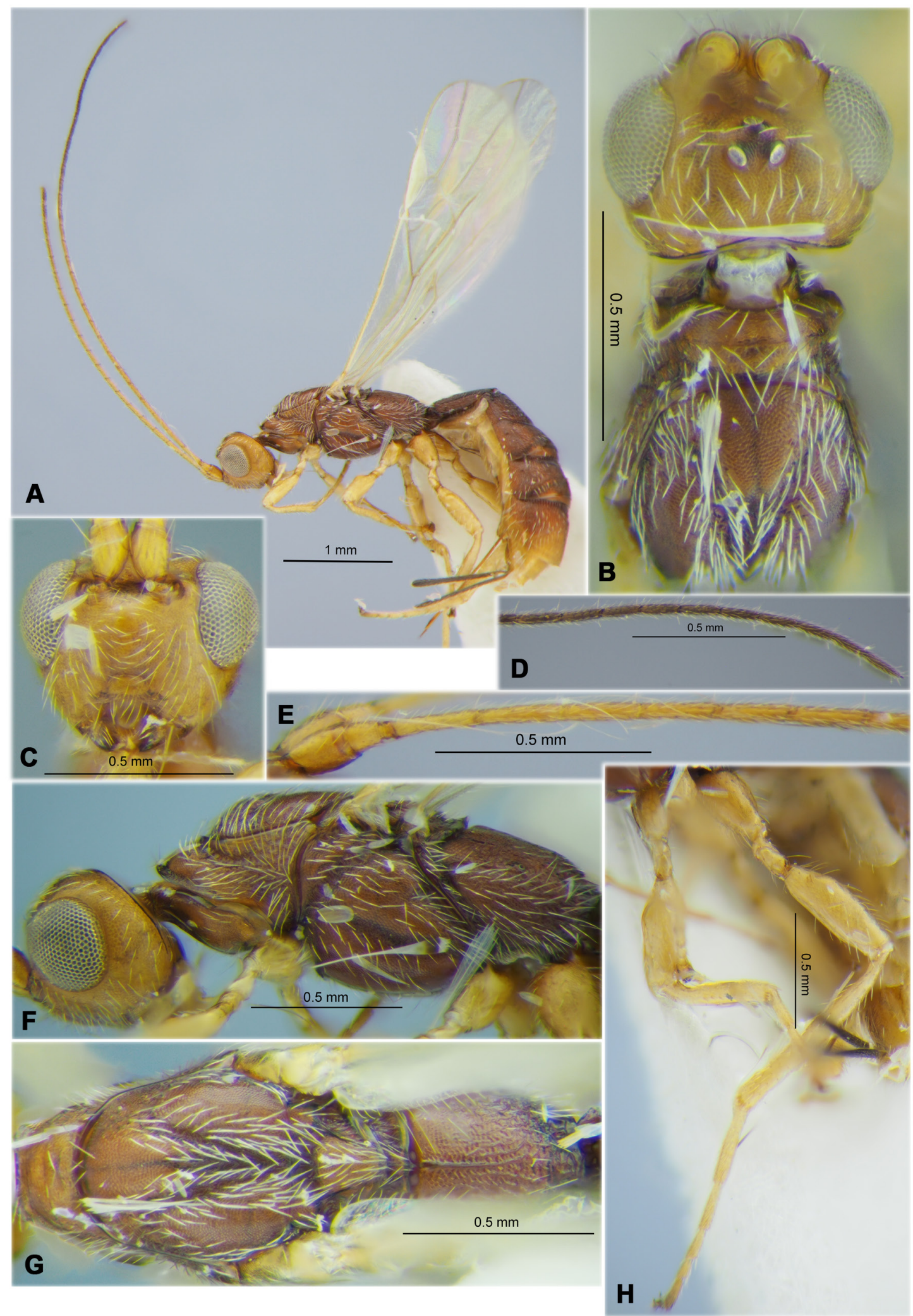

Fig. 53. Rhaconotus (Rh.) manolus Nixon, 1941, $q$ (RMNH). A. Habitus, lateral view. B. Head and mesoscutum, dorsal view. C. Head, front view. D. Apical segments of antenna. E. Basal segments of antenna. F. Head and mesosoma, lateral view. G. Mesosoma, dorsal view. H. Hind leg. 

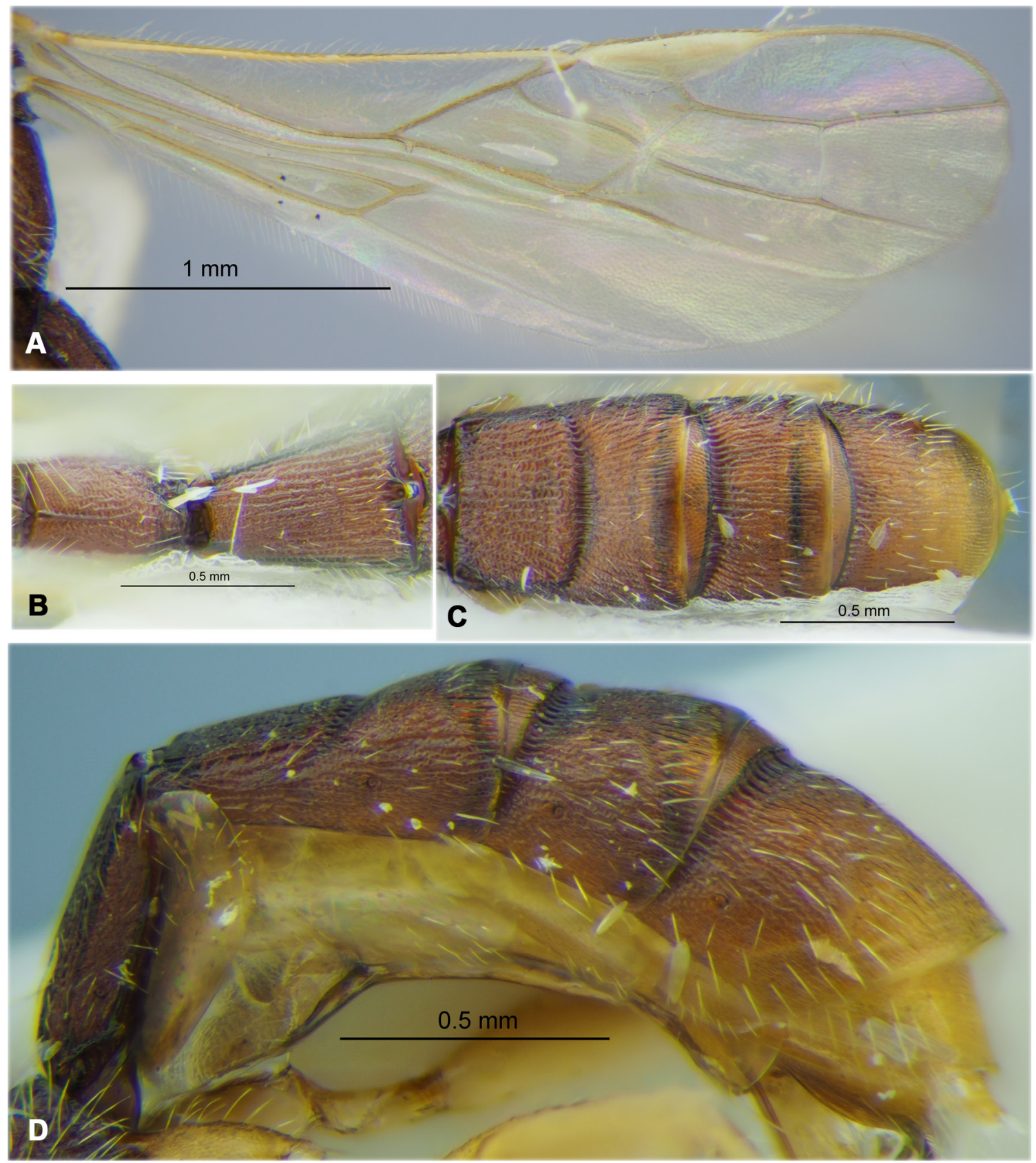

Fig. 54. Rhaconotus (Rh.) manolus Nixon, 1941, $q$ (RMNH). A. Wings. B. Propodeum and first metasomal tergite, dorsal view. C. Second to fifth metasomal tergites, dorsal view. D. Metasoma, lateral view. 
S. Al Haruri. RMNH'01"; RMNH • 1 đ̧; "Yemen, Al Kowd, viii.2000, light-tr., no 4952, A.v. Harten \& S. Al Haruri, RMNH'01"; RMNH - 2 o+ $O$; "Yemen (5750), Al Kowd, 8-12.vii.2001, light trap, A.v. Harten \& S. Al Haruri. RMNH'02"; RMNH • 1 क; "Yemen (6141), Al Kowd, 16-20.viii.2001, light trap, A.v. Harten \& S. Al Haruri. RMNH'02"; RMNH • 5 q o 우 "Yemen (8136), Al Kowd, ix.2003, light trap, A.v. Harten, S. Al Haruri, RMNH'03"; RMNH • 1 \%; same label data as for preceding; ZISP • 1 q; "Yemen, Ta'izz, viii.1999, light tr., no 4056, A.v. Harten \& A. Awad, RMNH'01"; RMNH • 2 우; "Yemen (5404), Hammam 'Ali, from coffee-berries (with Ceratitis capitata ?), 14.iii.2001, A.v. Harten, RMNH'02”; RMNH • 1 q; "Yemen (5962), 12 km NW Manakhah, Mal. trap; 3.vii-21.viii.2001, lighttrap, A.v. Harten, RMNH'02”; RMNH.

\section{Distribution}

South Africa, *UAE, *Yemen.

Rhaconotus (Rhaconotus) microexcavatus Belokobylskij sp. nov. urn:1sid:zoobank.org:act:4B0164DD-0977-49CF-8DE2-FCD562CABAF6

Figs $55-56$

\section{Comparative diagnosis}

This new species is similar to Rhaconotus bicoloricornis (Granger, 1949) from Madagascar (Granger 1949), but differs from the latter by the POL of ocelli almost equal to Od, about 0.5 times OOL ( 0.7 and 0.3 times accordingly in $R$. bicoloricornis); malar space height 0.35 times height of eye and almost equal to basal width of mandible ( 0.25 and 0.7 times accordingly in Rh. bicoloricornis); antenna about as long as body (1.6 times longer in Rh. bicoloricornis); mesosoma 2.4 times longer than its height (about 2.0 times in Rh. bicoloricornis); radial (marginal) cell weakly shortened, metacarpus (1-R1) 0.9 times as long as pterostigma (not shortened and metacarpus (1-R1) 1.3 times longer than pterostigma in Rh. bicoloricornis); second metasomal tergite with wide and sculptured posterior area and its median length 1.6 times length of remaining part of tergite (with short and smooth posterior area which is 0.6 times as long as length of remaining part of tergite in Rh. bicoloricornis); fifth tergite on posterior margin with low and small median incision (without such incision in Rh. bicoloricornis) and 1.7-1.8 times longer than fourth tergite (1.4 times in Rh. bicoloricornis); propodeum with short basal carina which is about 0.3 times as long as propodeum (long basal carina which is about 0.7 times as long as propodeum in Rh. bicoloricornis); and lateral lobes of mesoscutum widely glabrous (entirely setose in $R h$. bicoloricornis).

Rhaconotus microexcavatus sp. nov. is also similar with Rh. magniareolus sp. nov., but it differs from the latter in the temple shorter, transverse diameter of its eye 2.2-2.5 times temple length (1.4-1.7 times in Rh. magniareolus); upper margin of clypeus situated distinctly upper than lower level of eyes (on or weakly below in Rh. magniareolus); penultimate segment of antenna 4.0-4.5 times longer than wide (3.5-3.7 times in Rh. magniareolus); radial (marginal) cell of fore wing weakly shortened (not shortened in Rh. magniareolus); first metasomal tergite longer, 1.5-1.6 times longer than its posterior width (shorter, 1.2-1.4 times longer than its posterior width in Rh. magniareolus); posterior area of second tergite large, 1.6-1.8 times longer than remaining part of tergite (shorter, only 1.3-1.4 times in $R h$. magniareolus); second tergite 1.4-1.6 times longer than third tergite (2.6-3.3 times in Rh. magniareolus); fifth tergite on posterior margin with low and small median incision (without such incision in Rh. magniareolus); and ovipositor sheath longer, $1.1-1.2$ times longer than mesosoma, $0.50-0.55$ times as long as fore wing (shorter, 0.8-0.9 and 0.4 times respectively in Rh. magniareolus).

\section{Etymology}

This new species is named after the small median excavation on the posterior margin of the fifth metasomal tergite. 


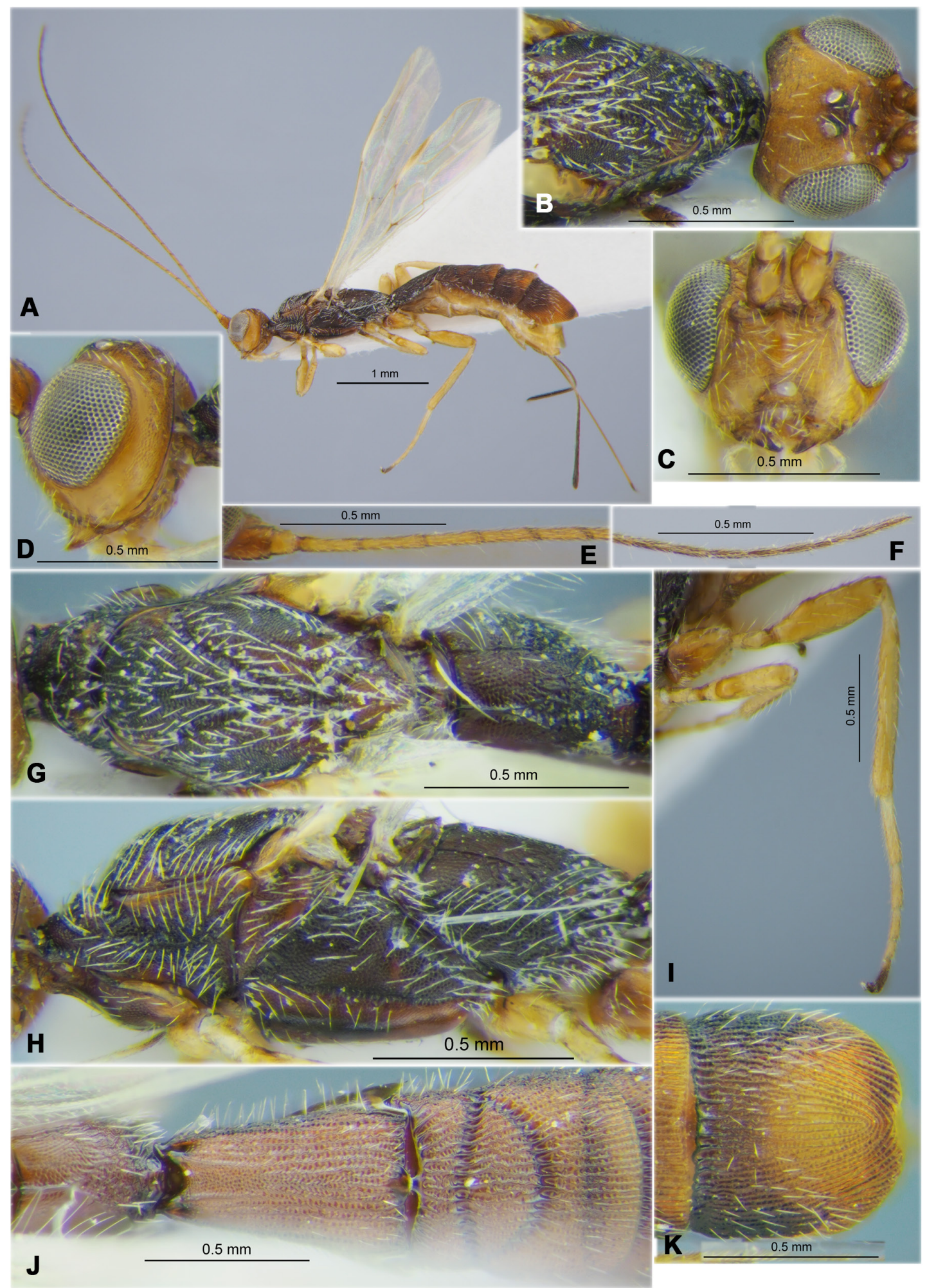

Fig. 55. Rhaconotus (Rh.) microexcavatus Belokobylskij sp. nov., holotype, $q$ (RMNH). A. Habitus, dorsal view. B. Head and mesoscutum, dorsal view. C. Head, front view. D. Head, lateral view. E. Basal segments of antenna. F. Apical segments of antenna. G. Mesosoma, dorsal view. H. Mesosoma, lateral view. I. Hind leg. J. Propodeum and first to third metasomal tergites, dorsal view. K. Fifth metasomal tergite, dorsal view. 


\section{Material examined}

Holotype

UNITED ARAB EMIRATES • $q$; “United Arab Emirates, Sharjah Desert Park (6491), light trap, 1-25. ii.2006, $25^{\circ} 17^{\prime} \mathrm{N}, 55^{\circ} 42^{\prime}$ E, A.v. Harten, RNMH'06”; RMNH.

\section{Paratypes}

UNITED ARAB EMIRATES • 1 \%; "United Arab Emirates, Sharjah Desert Park (1394), light tr., 13-23.

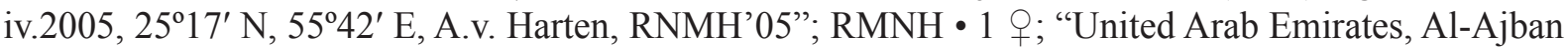

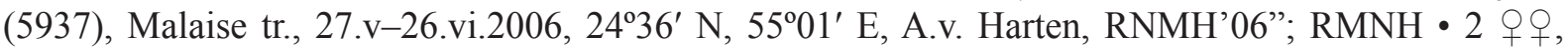
1 ơ; "United Arab Emirates, Sharjah Desert Park (2049-2074), light tr., 30.iv-31.v.2005, $25^{\circ} 17^{\prime}$ N, $55^{\circ} 42^{\prime}$ E, A.v. Harten, RNMH'05"; RMNH • 1 ; ; same label data as for preceding; ZISP • 1 (head missing); "United Arab Emirates, Sharjah Desert Park (1202), light tr., 29.iii-6.iv.2005, 25 $1^{\prime} \mathrm{N}, 55^{\circ} 4^{\prime}$ E. A.v. Harten, RMNH'06”; RMNH • 2 q $\%$; “United Arab Emirates, Sharjah Desert Park (1308), light tr., 6-13.iv.2005, $25^{\circ} 17^{\prime} \mathrm{N}, 55^{\circ} 42^{\prime}$ E, A.v. Harten, RNMH'05”; RMNH.

\section{Description}

\section{Female}

Measurements. Body length 3.5-4.2 mm; fore wing length 2.4-2.9 mm.

HEAD. Head width 1.6-1.7 times its median length, 1.3-1.4 times width of mesoscutum. Head behind eyes distinctly evenly convex-roundly narrowed; transverse diameter of eye 2.2-2.5 times larger than temple length. Ocelli medium-sized, arranged almost in equilateral triangle; POL 1.0-1.3 times Od, 0.50.7 times OOL. Eye glabrous, weakly emarginated opposite antennal sockets, 1.15-1.20 times as high as broad. Malar space height $0.35-0.40$ times height of eye, almost equal to basal width of mandible. Malar suture shallow but distinct. Face width about 0.9 times height of eye and 1.2-1.3 times height of face and clypeus combined. Upper margin of clypeus situated distinctly upper than lower level of eyes. Hypoclypeal depression round, its width $0.7-0.8$ times distance from edge of depression to eye, $0.35-$ 0.40 times width of face. Occipital carina not fused with hypostomal carina below being obliterated for short distance upper base of mandible. Vertex convex.

AnTENNA. Antenna long, slender, weakly setiform, 29-31-segmented, as long as body. Length of scape 1.7-1.8 times its maximum width. First flagellar segment 4.3-5.5 times longer than its apical width, 0.90-0.95 times as long as second segment. Penultimate segment 4.0-4.5 times longer than wide, 0.60.7 times as long as first segment, $0.8-0.9$ times as long as apical segment; latter acuminate apically and with short spine.

Mesosoma. Length 2.3-2.4 times its height. Pronotum anteriorly weakly or very weakly convex (dorsal view), weakly convex dorsally in posterior half (lateral view). Pronotal carina distinct and situated medially. Mesoscutum weakly and gently-roundly elevated above pronotum. Notauli deep, rather wide, complete and densely crenulate with reticulation. Mesoscutum without median longitudinal depression. Prescutellar depression long, rather deep, its posterior side distinctly and evenly curved, without median carina, with two additional carinae and weakly rugulose to smooth between carinae, $0.30-0.35$ times as long as convex scutellum. Precoxal sulcus distinct, rather deep at most anterior part and shallow in posterior one-fifth, sparsely crenulate with fine additional reticulation, almost straight, long, running along almost entire lower part of mesopleuron. Prepectal carina distinct, weakly widened below, with not large lobes opposite fore coxae. Metanotum with short, oblique, acuminate distally and rather wide basally dorsal process (lateral view). Metapleural lobe distinct, wide, rounded distally.

WINGS. Length of fore wing 3.9-4.5 times its maximum width. Radial (marginal) cell weakly shortened, 3.7-4.2 times longer than maximum width. Metacarpus (1-R1) 0.9-1.0 times as long as pterostigma. 
Radial vein (r) arising weakly before middle of pterostigma. First radial abscissa (r) forming very obtuse angle with second abscissa (3-SR). Second radial abscissa (3-SR) 2.7-3.0 times longer than first abscissa (r), 0.60-0.65 times as long as the weakly curved third abscissa (SR1), 1.6-1.9 times longer than first radiomedial vein (2-SR). Second radiomedial (submarginal) cell rather long, not widened distally, its length 3.4-3.6 times maximum width, 1.2-1.3 times longer than rather narrow brachial (subdiscal) cell. First medial abscissa $(1-\mathrm{SR}+\mathrm{M})$ distinctly sinuate. Recurrent vein $(\mathrm{m}-\mathrm{cu})$ postfurcal. Mediocubital
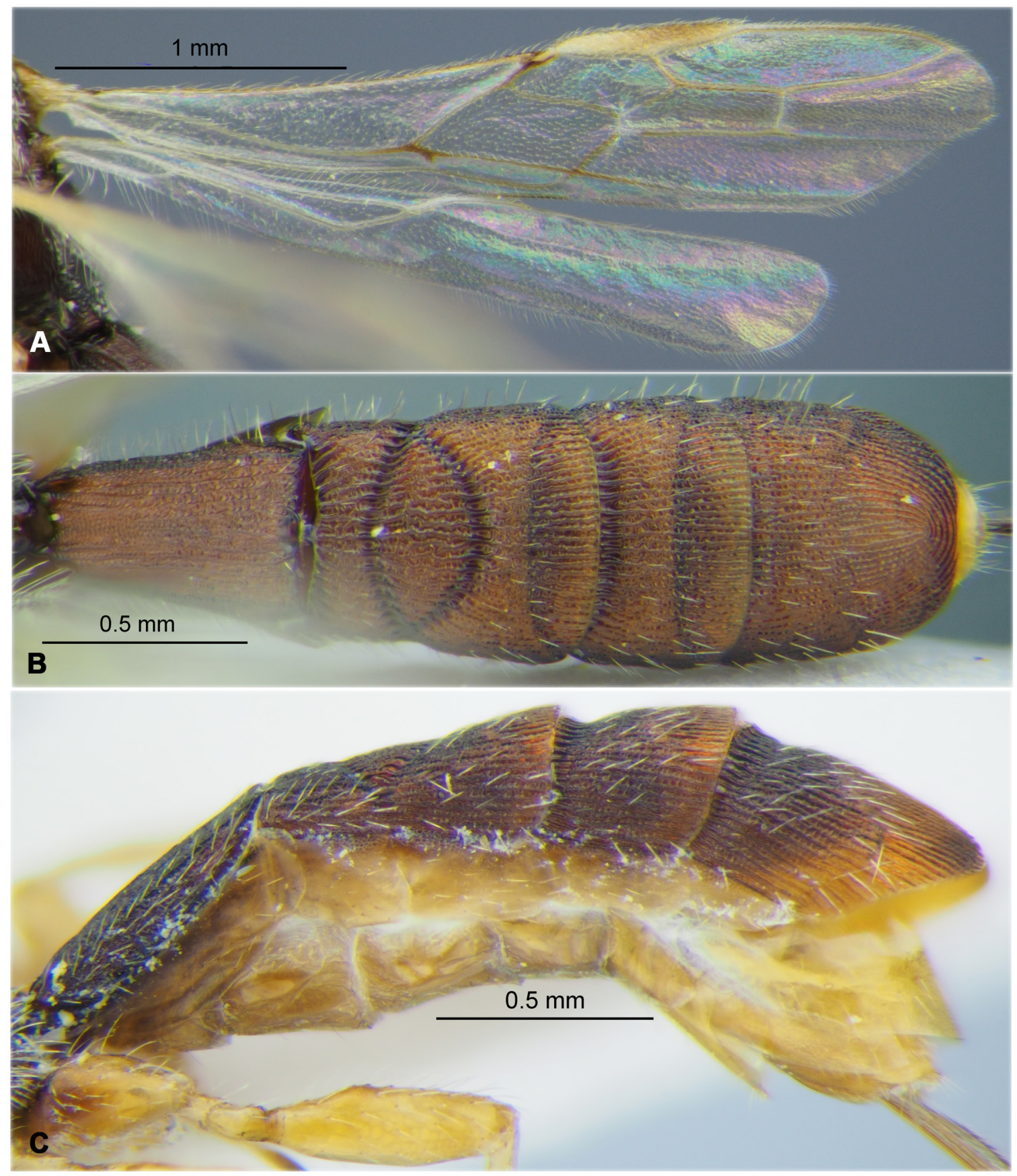

Fig. 56. Rhaconotus (Rh.) microexcavatus Belokobylskij sp. nov., holotype, $q$ (RMNH). A. Wings. B. Metasoma, dorsal view. C. Metasoma, lateral view. 
vein $(M+C U 1)$ weakly curved to anal vein (1-1A) in distal half. Distance from nervulus (cu-a) to basal vein (1-M) 1.2-1.4 times nervulus (cu-a) length. Brachial (subdiscal) cell gently and roundly closed weakly before level of recurrent vein (m-cu); posterior bulla on brachial vein (CU1b) present and short; posterior abscissa of anal vein (1-1A) (behind brachial vein (CU1b)) absent. Hind wing not narrowed basally, 6.0-7.0 times longer than wide. First costal abscissa $(\mathrm{C}+\mathrm{SC}+\mathrm{R}) 0.45-0.55$ times as long as second abscissa $(1-\mathrm{SC}+\mathrm{R})$. First abscissa of mediocubital vein $(\mathrm{M}+\mathrm{CU}) 0.5-0.6$ times as long as second abscissa (1-M). Recurrent vein (m-cu) short, unsclerotised, antefurcal, oblique, straight.

Legs. Hind femur with distinct and wide basodorsal protuberance, its length 3.1-3.3 times maximum width. Hind tarsus about as long as hind tibia. Hind tibia distally with four outside spines. Hind basitarsus $0.45-0.55$ times combined length of second-fifth segments. Second tarsal segment $0.6-0.7$ times as long as basitarsus, 1.1-1.4 times longer than fifth segment (without pretarsus).

Metasoma. Metasoma 1.2-1.3 times longer than head and mesosoma combined, with five dorsally visible hardly sclerotised tergites. Maximum subposterior width of first tergite 2.0-2.2 times its anterior width; its length 1.5-1.6 times posterior width. Second tergite without anterior area, with long and wide posterior area separated anteriorly by deep and evenly curved crenulate transverse furrow. Median length of posterior area 1.6-1.8 times median length of remaining part of second tergite. Median length of second tergite (with posterior area) $0.8-0.9$ times its anterior width, 1.4-1.6 times length of third tergite. Second suture deep and strongly evenly curved. Third and fourth tergites without posterior transverse furrow; fourth and fifth tergites with coarse crenulate and deep anterior transverse furrow. Fifth tergite enlarged, on posterior margin distinctly convexly curved, with low and small median incision, without tubercles laterally. Fifth tergite 1.7-1.8 times longer than fourth tergite. Ovipositor sheath $0.70-0.75$ times as long as metasoma, $1.1-1.2$ times longer than mesosoma, $0.50-0.55$ times as long as fore wing.

SCULPTURE AND PUBESCENCE. Vertex entirely finely and almost evenly transversely striate with very dense reticulation or granulation between striae; frons entirely and distinctly reticulate-striate; temple mainly reticulate-coriaceous and almost smooth below. Face densely reticulate-granulate medially, finely coriaceous to smooth in wide lower lateral parts. Mesoscutum densely reticulate-rugulose, sculpture often situated in rather even curved and dense transverse lines, with rugosity in narrow area in medio-posterior third. Scutellum densely small reticulate. Mesopleuron densely reticulate-areolate, finely reticulatecoriaceous below precoxal sulcus. Propodeum with basolateral areas and areola delineated by rather distinct carinae, basolateral areas entirely densely reticulate-areolate, remainder of propodeum together with areola densely and rather coarsely rugose; basal carina $0.7-1.0$ times as long as basolateral fork of areola; areola rather narrow and long, about 2.0 times longer than wide. Hind coxa densely reticulateareolate, almost coriaceous laterally; hind femur densely reticulate in dorsal half and almost smooth in ventral half. First tergite with distinctly and subparallel dorsal carinae at least in anterior half. First to fifth tergites distinctly and densely striate, with distinct and dense rugulosity between striae; striae on posterior half of fifth tergite weakly curved. Second-fifth tergites laterally with distinct dense striae and rugosity between it. Vertex with short and rather sparse semi-erect white setae directed forwards laterally and in posterior third, almost glabrous medio-anteriorly. Mesoscutum on median lobe often entirely or at least latero-posteriorly and on lateral lobes near notauli and laterally with rather dense and semi-erect white setae, medial parts of lateral lobes always widely glabrous, and sometimes medio-anterior part of medial lobe glabrous also. Mesopleuron almost entirely and sparse with distinct white setae. Metasoma dorsally with short and rather sparse semi-erect white setae on most part. Hind tibia dorsally with semi-erect and rather dense short white setae, length of these setae $0.5-1.1$ times maximum width of hind tibia.

CoLour. Head mainly brownish yellow or light brown, infuscate dorsally. Mesosoma and metasoma dark reddish brown to almost black in some places, partly reddish brown, metasomal third-fifth tergites posteriorly reddish brown to light reddish brown, posterior tergites and sternites brownish yellow. Scape 
and pedicel of antenna light reddish brown, basal third or quarter of flagellum yellow to yellowish brown, remaining long apical part of flagellum brown to almost black. Palpi pale yellow or yellow. Legs mainly yellow with brownish tint or brownish yellow, and darker basally, all fifth tarsal segments mainly dark brown. Ovipositor sheath yellow in anterior third and black in posterior two-thirds. Fore wing subhyaline. Pterostigma mainly brown of light reddish brown, pale yellow in basal third.

\section{Male}

Body length $3.3 \mathrm{~mm}$; fore wing length $2.3 \mathrm{~mm}$. Transverse diameter of eye 2.1 times larger than temple length. Antenna 26-segmented, weakly longer than body. Body 2.5 times longer than maximum height. Hind femur slender, its length 3.4 times maximum width. Metasoma 1.4 times longer than head and mesosoma combined, with 6 dorsally visible and hardly sclerotised tergites. Maximum subposterior width of first tergite 1.7 times its anterior width; its length 1.8 times posterior width. Median length of posterior area of second tergite 0.7 times median length of remaining part of second tergite. Median length of second tergite (with posterior area) 1.2 times its anterior width, 1.4 times length of third tergite. Fifth and sixth without median incisure on their posterior margins. Sixth tergite enlarged, on posterior margin distinctly convexly curved, weakly narrowed distally, almost as long as fifth tergite, 1.1 times longer than fourth tergite. Otherwise similar to female.

\section{Distribution}

UAE.

Rhaconotus (Rhaconotus) scirpophagae Wilkinson, 1927

Figs $57-58$

Rhaconotus scirpophagae Wilkinson, 1927: 34.

Rhaconotus scirpophagae-Nixon 1939: 123; 1941:482. — Polaszek et al. 1994: 80. — van Achterberg \& Polaszek 1996: 59. — Belokobylskij 2001b: 133. — Yu et al. 2016.

\section{Material examined}

YEMEN • 1 क; "Yemen (4054), Al Kowd, viii.1999, light-trap, A.v. Harten \& S. Al Haruri. RMNH’00”; RMNH.

\section{Hosts}

Chilo auricilius Dudgeon, 1905, Ch. partellus (Swinhoe, 1885), Scirpophaga excerptalis (Walker, 1863), S. nivella (Fabricius, 1794) (Crambidae); Busseola fusca (Fuller, 1901) (Noctuidae); Maliarpha separatella Ragonot, 1888 (Pyralidae) (Lepidoptera).

\section{Distribution}

Oriental: Bangladesh, China (Taiwan, introduced), India, Indonesia, Pakistan, Vietnam; Afrotropical: Ghana, Ivory Coast, Kenya, Mauritius (introduced), Nigeria, Senegal, Sierra Leone, Tanzania, *Yemen; Neotropical: Trinidad \& Tobago (introduced).

Rhaconotus (Rhaconotus) sudanensis Wilkinson, 1927

Figs 59-60

Rhaconotus sudanensis Wilkinson, 1927: 36.

Rhaconotus sudanensis - Nixon 1941: 489. - Yu et al. 2016.

Rhaconotus carinatus - Edmardash et al. 2020: 58 (auct.). 


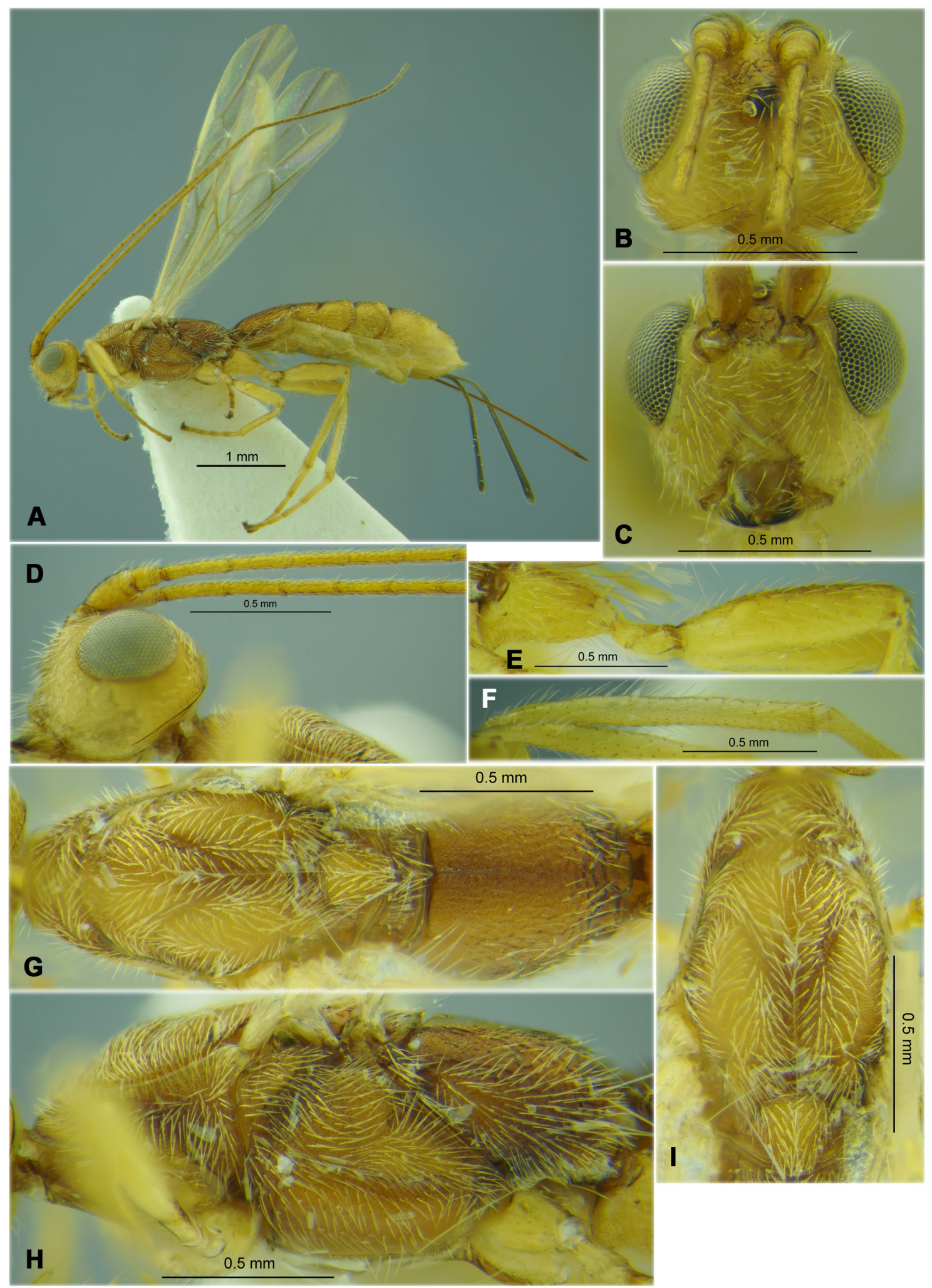

Fig. 57. Rhaconotus (Rh.) scirpophagae Wilkinson, 1927, q (RMNH). A. Habitus, dorsal view. B. Head, dorsal view. C. Head, front view. D. Head and basal segments of antenna, lateral view. E. Hind coxa and femur. F. Hind tibia. G. Mesosoma, dorsal view. H. Mesosoma, lateral view. I. Mesoscutum and scutellum, dorsal view. 

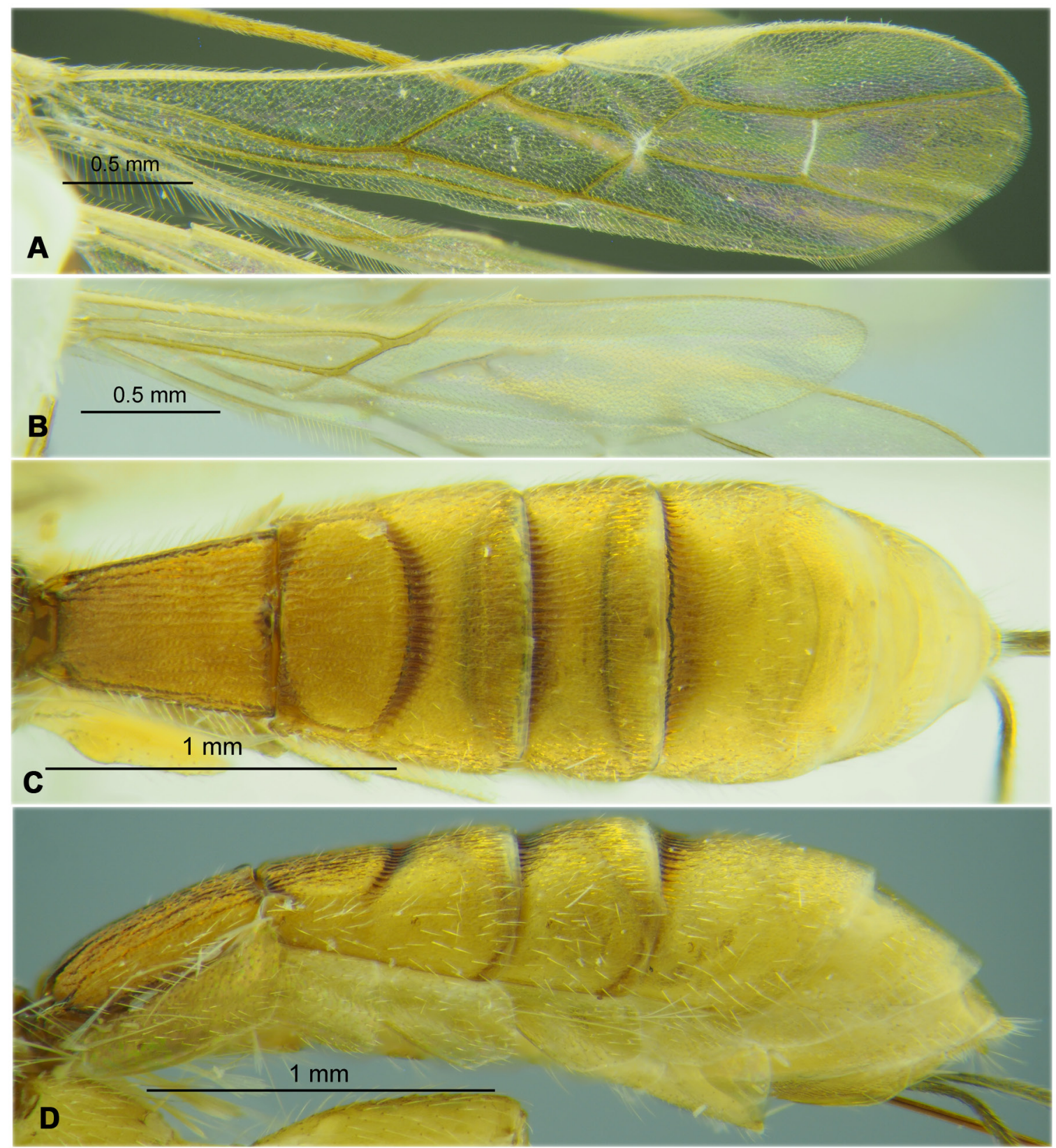

Fig. 58. Rhaconotus (Rh.) scirpophagae Wilkinson, 1927, q (RMNH). A. Fore wing. B. Hind wing. C. Metasoma, dorsal view. D. Metasoma, lateral view. 


\section{Material examined}

UNITED ARAB EMIRATES • 1 \%; "United Arab Emirates, Fujairah (1314), light tr., 13-19.iv.2005, $25^{\circ} 0^{\prime}$ N, $56^{\circ} 2^{\prime}$ E. A.v. Harten, RMNH'05"; RMNH 1 q; "United Arab Emirates, Sharjah Desert Park

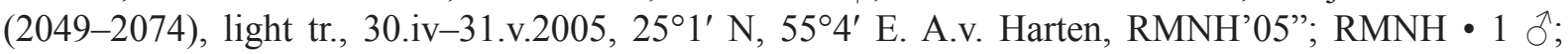
"United Arab Emirates, Wadi Safad (3121-3130), light tr., 26.xii.2005-2.i.2006, 251' N, 56 $6^{\circ} 1^{\prime}$ E, A.v. Harten, RNMH'06"; RMNH - 1 क; "United Arab Emirates, Wadi Safad (5550), at light, 1-8. vii.2006, $25^{\circ} 1^{\prime} \mathrm{N}, 56^{\circ} 1^{\prime}$ E, A.v. Harten, RNMH'06"; RMNH • 1 q; same label data as for preceding;

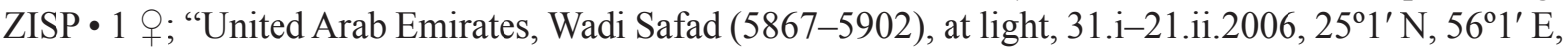
A.v. Harten, RNMH'06"; RMNH - 1 q; "United Arab Emirates, Al-Ajban (5937), Malaise tr., 27.v-26.

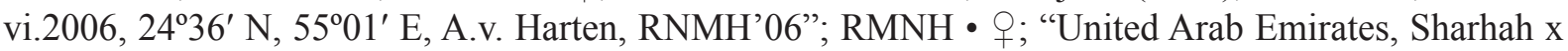
Khor Kalba (6319-6320), light trap, 16-31.i.2006, A.v. Harten, RNMH'06”; RMNH • 1 क; "United Arab Emirates, Sharhah x Khor Kalba (6327-6337), light trap, 24-30.vi.2006, A.v. Harten, RMNH'06";

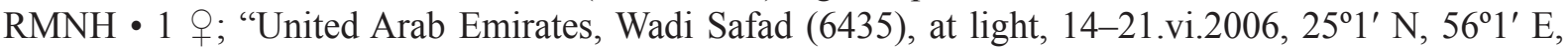
A.v. Harten, RNMH'06"; RMNH - 1 क; "United Arab Emirates, Wadi Maidaq (6474), light trap, 27.iv4.v.2006, $25^{\circ} 1^{\prime} \mathrm{N}, 56^{\circ} 0^{\prime}$ E, A.v. Harten, RNMH'07”; RMNH • 1 o ; same label data as for preceding; ZISP • 1 क ; "United Arab Emirates, Sharhah x Khor Kalba (6599), light trap, 24-30.v.2006, A.v. Harten, RMNH'06”; RMNH.

YEMEN • 4 우, 1 ठ; ; "Yemen (no 2989), Ta'izz, light trap, 1-3.iv.1998, A.v. Harten \& Ahmad Ahwad, RMNH'98"; RMNH • 2 ㅇ; ; same label data as for preceding; ZISP • 1 q; "Yemen (no 3111), Ta'izz, light trap, 26-28.v.1998, A.v. Harten \& Ahmad Ahwad, RMNH'98"; RMNH • 1 \%; "Yemen (3698), Sana'a, v.1999, Light-trap, A.v. Harten, RMNH'00"; RMNH • 1 q; "Yemen (4054), Al Kowd, viii.1999, light-trap, A.v. Harten \& S. Al Haruri, RMNH'00"; RMNH • 2 우; "Yemen (4055), Al Kowd, ix.1999, light trap, A.v. Harten \& S. Al Haruri. RMNH'00"; RMNH • 3 q $ᄋ$; "Yemen, Al Kowd, v-vi.2000, light tr., no. 4719, A.v. Harten \& S. Al Haruri. RMNH'01"; RMNH • 1 क; "Yemen, Lahj, xi-xii.1999, Mal. tr., no 4291, A.v. Harten \& A. Sallam, RMNH'02"; RMNH • 1 q; "Yemen (5404), Hammam 'Ali, from coffee-berries (with Ceratitis capitata ?), 14.iii.2001, A.v. Harten, RMNH'02"; RMNH • 1 q; "Yemen, Al Kowd, ii.2001, light tr., no. 5484, A.v. Harten \& S. Al Haruri, RMNH'01"; RMNH • 1 O; "Yemen (5700), Ar Rujum, 9.iv-5.vi.2001, Mal. trap; A.v. Harten RMNH'02"; RMNH • 1 क; "Yemen (5750), Al Kowd, 8-12.vii.2001, light trap, A.v. Harten \& S. Al Haruri. RMNH'02"; RMNH • 1 \&, 1 ð’; "Yemen (6158), Al Lahima, 17.ix-14.ii.2001, Malaise trap, A.v. Harten, RMNH'02”; RMNH.

\section{Hosts}

Sphenoptera gossypii Kerremans, 1892, S. neglecta (Klug, 1835) (Coleoptera: Buprestidae); Coniesta ignefusalis (Hampson, 1919) (Lepidoptera: Crambidae).

\section{Distribution}

*Saudi Arabia, Senegal, Sudan, *UAE, *Yemen.

\section{Remarks}

After careful study of the photos of the Rhaconotus species determined as Rh. carinatus Polaszek, 1994 by Edmardash et al. (2020), and the examination of numerous specimens from the UAE and Yemen, we are now sure that the specimen listed by Edmardash et al. (2020) is actually Rh. sudanensis. Therefore, Rh. carinatus has to be excluded both from the faunistic list of Saudi Arabia and of the Arabian Peninsula.

Sometimes, a few specimens from Yemen are distinctly infuscate with the head dorsally dark brown to almost black. Additionally, a few specimens from Yemen have a slightly longer ovipositor, with the sheath as long as or only weakly shorter than length of metasoma. 


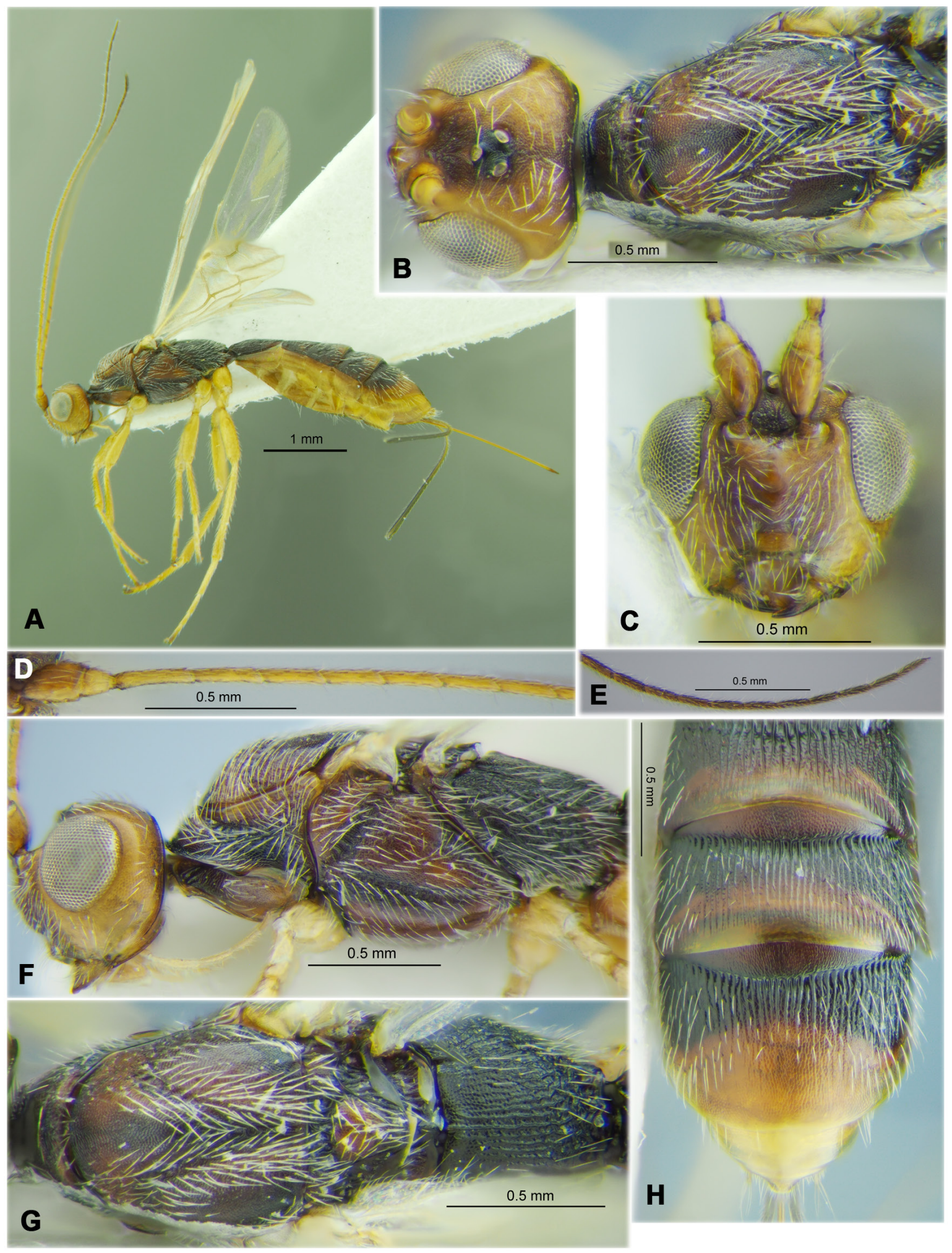

Fig. 59. Rhaconotus $(R h$.$) sudanensis Wilkinson, 1927, q$ (RMNH). A. Habitus, lateral view. B. Head and mesoscutum, dorsal view. C. Head, front view. D. Basal segments of antenna. E. Apical segments of antenna. F. Head and mesosoma, lateral view. G. Mesosoma, dorsal view. H. Third to fifth metasomal tergites, dorsal view. 

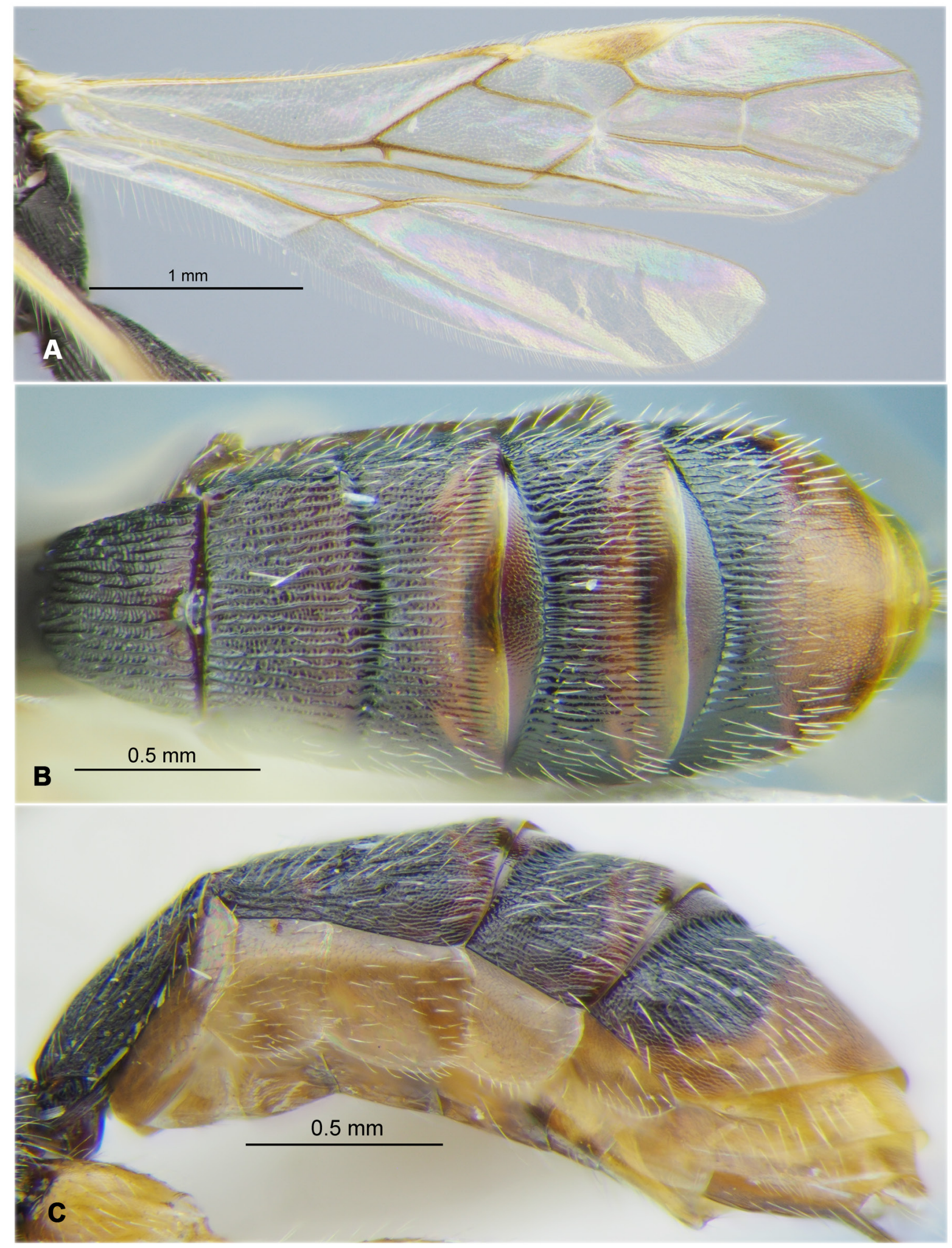

Fig. 60. Rhaconotus (Rh.) sudanensis Wilkinson, 1927, $q$ (RMNH). A. Wings. B. Metasoma, dorsal view. C. Metasoma, lateral view. 
Rhaconotus (Rhaconotus) vanharteni Belokobylskij sp. nov. urn:lsid:zoobank.org:act:6855ED2E-04FA-4429-B3DF-ECED89F62A94

Figs 61-62

\section{Comparative diagnosis}

This species is morphologically similar to Rhaconotus dentatus (Brues, 1924) from South Africa, but differs from the latter in having the pronotum anteriorly in dorsal view straight (convex in Rh. dentatus), posteriorly strongly and narrowly convex dorsally in lateral view (regularly convex dorsally in $R h$. dentatus), prescutellar depression with single median carina (with four carinae in Rh. dentatus), second radial abscissa of fore wing 1.9 times longer than first abscissa and 1.6 times longer than first radiomedial vein (3.1 and almost twice accordingly in $R h$. dentatus), median length of second metasomal tergite 0.4 times its anterior width and equal to length of third tergite ( 0.7 and 1.3 times accordingly in Rh. dentatus), fifth tergite (with processes), 2.6 times longer than fourth tergite (1.7 times in Rh. dentatus), mesosoma and metasoma reddish brown (almost black in Rh. dentatus), hind coxa and femur mainly yellowish brown (mainly dark brown to black in $R h$. dentatus).

\section{Etymology}

This species is named in honour to the collector of this species, A. van Harten.

\section{Material examined}

\section{Holotype}

YEMEN • +; "Yemen, Al Kowd, v-vi.2000, light tr., No 4719, A.v. Harten \& S, Al Haruri, RMNH'01”; RMNH.

\section{Description}

Female

MEASUREMENTS. Body length $3.3 \mathrm{~mm}$; fore wing length $2.5 \mathrm{~mm}$.

HEAD. Head width 1.5 times its median length, 1.3 times width of mesoscutum. Head behind eyes evenly roundly narrowed; transverse diameter of eye 2.0 times larger than temple length. Ocelli medium-sized, arranged in triangle with base 1.2 times its sides; POL 1.6 times Od, 0.8 times OOL. Eye glabrous, very weakly emarginated opposite antennal sockets, 1.2 times as high as broad. Malar space height 0.35 times height of eye, $0.8-0.9$ times basal width of mandible. Malar suture very fine, subvertical and weakly curved. Face width almost equal to height of eye and 1.5 times height of face and clypeus combined. Upper margin of clypeus situated weakly upper than lower level of eyes. Hypoclypeal depression round, its width 0.6 times distance from edge of depression to eye, 0.3 times width of face. Occipital carina not fused with hypostomal carina below being obliterated for short distance upper base of mandible. Vertex convex.

AntenNA. Antenna filiform, more than 23-segmented (apical segments missing). Length of scape 1.6 times its maximum width. First flagellar segment about 4.0 times longer than its apical width, 0.9 times as long as second segment. Subapical segment 3.5 times longer than wide.

Mesosoma. Length 2.2 times its height. Pronotum anteriorly straight (dorsal view), posteriorly strongly and narrowly convex dorsally (lateral view). Pronotal carina distinct, widely separated from posterior margin of pronotum, distances from carina to anterior and posterior margins of pronotum subequal. Mesoscutum weakly and gently-roundly elevated above pronotum. Notauli rather deep, complete and crenulated. Mesoscutum without median longitudinal depression. Prescutellar depression rather short, rather deep, its posterior sides straight obliquely directed posterolaterally, with distinct median carina, 
sparsely and finely sculptured, 0.3 times as long as convex scutellum. Precoxal sulcus distinct, crenulatereticulate, straight, long, running along entire lower part of mesopleuron. Prepectal carina rather strong, not widened below, without lobes opposite fore coxae. Metanotum with short, slender and pointed tooth directed posteriorly. Metapleural lobe rather short, wide, rounded distally.

Wings. Length of fore wing 4.0 times its maximum width. Radial (marginal) cell not shortened. Metacarpus (1-R1) 1.15 times longer than pterostigma. Radial vein (r) arising weakly before middle of pterostigma. First radial abscissa (r) forming very obtuse angle with second abscissa (3-SR). Second radial abscissa (3-SR) 1.9 times longer than first abscissa (r), 0.5 times as long as third abscissa (SR1), 1.6 times longer than first radiomedial vein (2-SR). Second radiomedial (submarginal) cell not widened distally, its length about 3.0 times maximum width, 1.1 times longer than brachial (subdiscal) cell. First medial abscissa $(1-\mathrm{SR}+\mathrm{M})$ weakly sinuate. Recurrent vein $(\mathrm{m}-\mathrm{cu})$ weakly postfurcal. Mediocubital vein $(\mathrm{M}+\mathrm{CU} 1)$ weakly curved to anal vein (1-1A) in distal half. Distance from nervulus (cu-a) to basal vein (1-M) almost equal to nervulus (cu-a) length. Brachial (subdiscal) cell gently and roundly closed weakly before level of recurrent vein (m-cu); posterior bulla on brachial vein (CU1b) present; posterior abscissa of anal vein (1-1A) (behind brachial vein (CU1b)) absent. Hind wing not narrowed basally, 5.4 times longer than wide. First costal abscissa $(\mathrm{C}+\mathrm{SC}+\mathrm{R}) 0.55$ times as long as second abscissa $(1-\mathrm{SC}+\mathrm{R})$. First abscissa of mediocubital vein $(\mathrm{M}+\mathrm{CU}) 0.6$ times as long as second abscissa $(1-\mathrm{M})$. Recurrent vein (m$\mathrm{cu})$ long and unsclerotized.

Legs. Hind femur with distinct baso-dorsal protuberance, its length 2.7 times maximum width. Hind tarsus as long as hind tibia. Hind tibia posteriorly with 4 outside spines. Hind basitarsus 0.45 times combined length of second-fifth segments. Second tarsal segment 0.6 times as long as basitarsus, 1.8 times longer than fourth segment, as long as fifth segment (without pretarsus).

Metasoma. Metasoma 1.2 times longer than head and mesosoma combined, with five dorsally visible hard tergites. Posterior width of first tergite 2.2 times its anterior width; its length 1.35 times posterior width. Second tergite without anterior and posterior areas, without additional transverse furrow. Median length of second tergite 0.4 times its anterior width, equal to length of third tergite. Second suture deep and distinct. Fifth tergite large, on posterior margin with two wide and rather long rounded posteriorly submedian processes, additionally with two short and wide rounded tubercles laterally. Fifth tergite (with processes) 2.6 times longer than fourth tergite. Ovipositor sheath 0.8 times as long as metasoma, 1.3 times longer than mesosoma, 0.65 times as long as fore wing.

SCulpture And pubescence. Vertex and frons distinctly and densely granulate-coriaceous, without striation or rugosity; temple finely reticulate-coriaceous in upper half, almost smooth in lower half. Face densely and finely reticulate-coriaceous, smooth in lower lateral parts. Mesoscutum densely granulate, without striation along notauli, with two subparally and almost straight striae in medio-posterior third. Scutellum granulate-coriaceous. Mesopleuron entirely densely reticulate-coriaceous, finely sculptured below precoxal sulcus. Propodeum with areas delineated by distinct carinae, with median carina in anterior half, areola rather short, pentagonal, rugose-granulate; long basolateral areas densely reticulatecoriaceous, without striation along carinae. Legs finely coriaceous in upper halves and almost smooth in lower halves. First tergite with distinct dorsal carinae in anterior half. First to fifth tergites distinctly, densely and at least partly undulately striate, with fine and dense rugulosity between striae. Second-fifth tergites laterally with distinct striae and very fine rugosity between it. Vertex with short and sparse semierect white setae directed backwards. Mesoscutum almost entirely with rather dense and semi-erect white setae, its lateral lobes glabrous on narrow lateral parts. Metasoma dorsally almost entirely with short and rather dense semi-erect white setae directed backwards. Hind tibia dorsally with almost erect and rather dense white setae, length of these setae 0.7-0.8 times maximum width of hind tibia. 


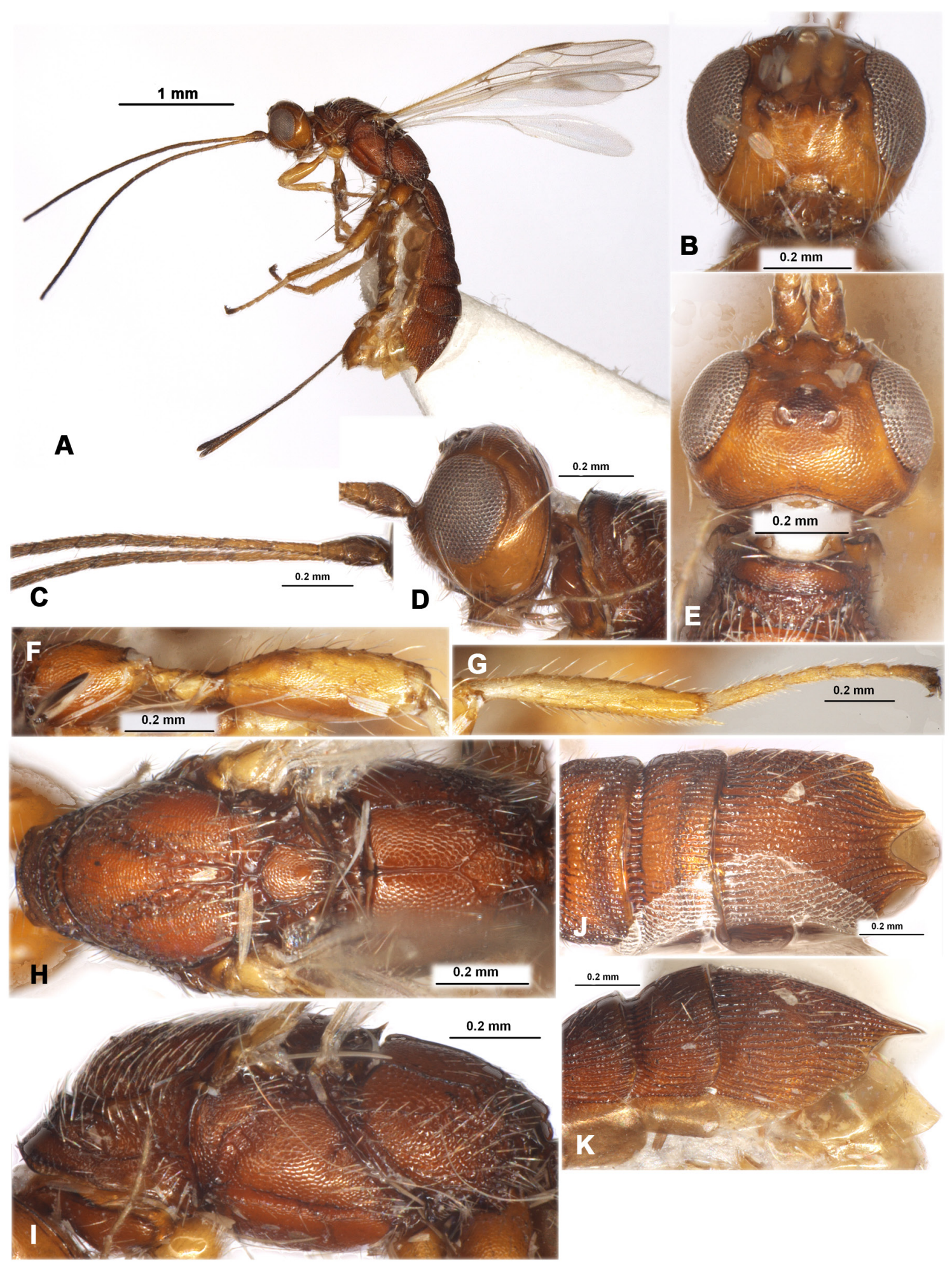

Fig. 61. Rhaconotus (Rh.) vanharteni Belokobylskij sp. nov., holotype, $q$ (RMNH). A. Habitus, lateral view. B. Head, front view. C. Basal segments of antenna. D. Head, lateral view. E. Head and anterior part of mesosoma, dorsal view. F. Hind coxa and femur. G. Hind tibia and tarsus. H. Mesosoma, dorsal view. I. Mesosoma, lateral view. J. Third to fifth metasomal tergites, dorsal view. K. Third to fifth metasomal tergites, lateral view. 

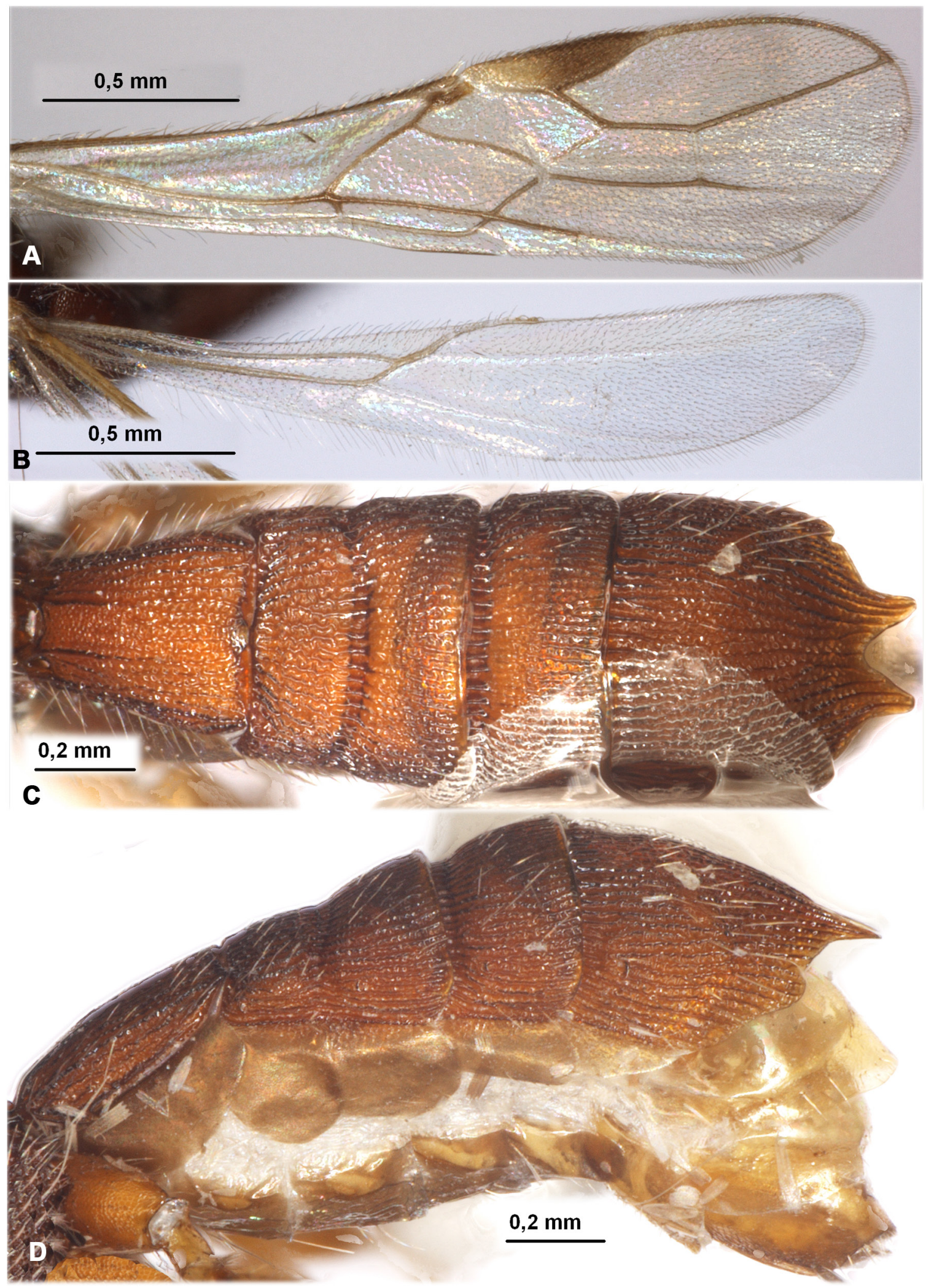

Fig. 62. Rhaconotus (Rh.) vanharteni Belokobylskij sp. nov., holotype, + (RMNH). A. Fore wing. B. Hind wing. C. Metasoma, dorsal view. D. Metasoma, lateral view. 
Colour. Head light reddish brown, widely yellowish anteriorly and below; mesosoma and metasoma reddish brown, segments behind fifth one yellowish brown. Scape reddish brown, pedicel and three flagellar segments yellowish brown, remaining part of flagellum brown to dark brown. Palpi brownish yellow to yellow. All legs yellowish brown, all tibiae basally paler, all tarsi yellowish, but last fifth segments dark. Ovipositor sheath dark brown to black. Fore wing almost hyaline. Pterostigma brown, yellow in basal third.

Male

Unknown.

\section{Distribution}

Yemen.

Tribe Ecphylini Hellén, 1957

Genus Aivalykus Nixon, 1938

\section{Type species}

Aivalykus eclectes Nixon, 1938, by original designation.

\section{Remarks}

This is a relatively small genus with almost worldwide distribution. It was recently recorded for the Arabian Peninsula (Ranjith et al. 2020).

\section{Distribution}

Afrotropical, Nearctic, Neotropical, Oceanic, Oriental and Palaearctic regions.

Aivalykus microaciculatus Ranjith \& Belokobylskij, 2020

Figs 63-64

Aivalykus microaciculatus Ranjith \& Belokobylskij in Ranjith et al., 2020: 271.

\section{Material examined}

Paratypes

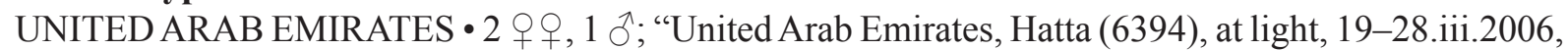
$24^{\circ} 4^{\prime} \mathrm{N}, 56^{\circ} 0^{\prime}$ E, A.v. Harten, RNMH'06”; RMNH • 1 q, 1 \%; same label data as for preceding; ZISP.

\section{Distribution}

India, UAE.

\section{Discussion}

This paper represents the first comprehensive taxonomic study of the braconid subfamily Doryctinae of the dry (desert and semi-desert) territories of the Arabian Peninsula. The study is based on a large number of specimens from the United Arab Emirates and Yemen, which were collected during almost 20 years. A total of 17 doryctine genera (Aivalykus, Dendrosotinus, Doryctes, Doryctophasmus, Hecabalodes, Hemidoryctes, Hemispathius, Heterospilus, Leluthia, Mimodoryctes, Neoheterospilus, Parallorhogas, Parana, Platyspathius, Rhaconotinus, Rhaconotus and Spathius) from six tribes (Doryctini, Ecphylini, Hecabolini, Heterospilini, Rhaconotini and Spathiini) and 38 species (including 20 species new for science) are recorded for the Arabian Peninsula in the current paper. According to our results, the 


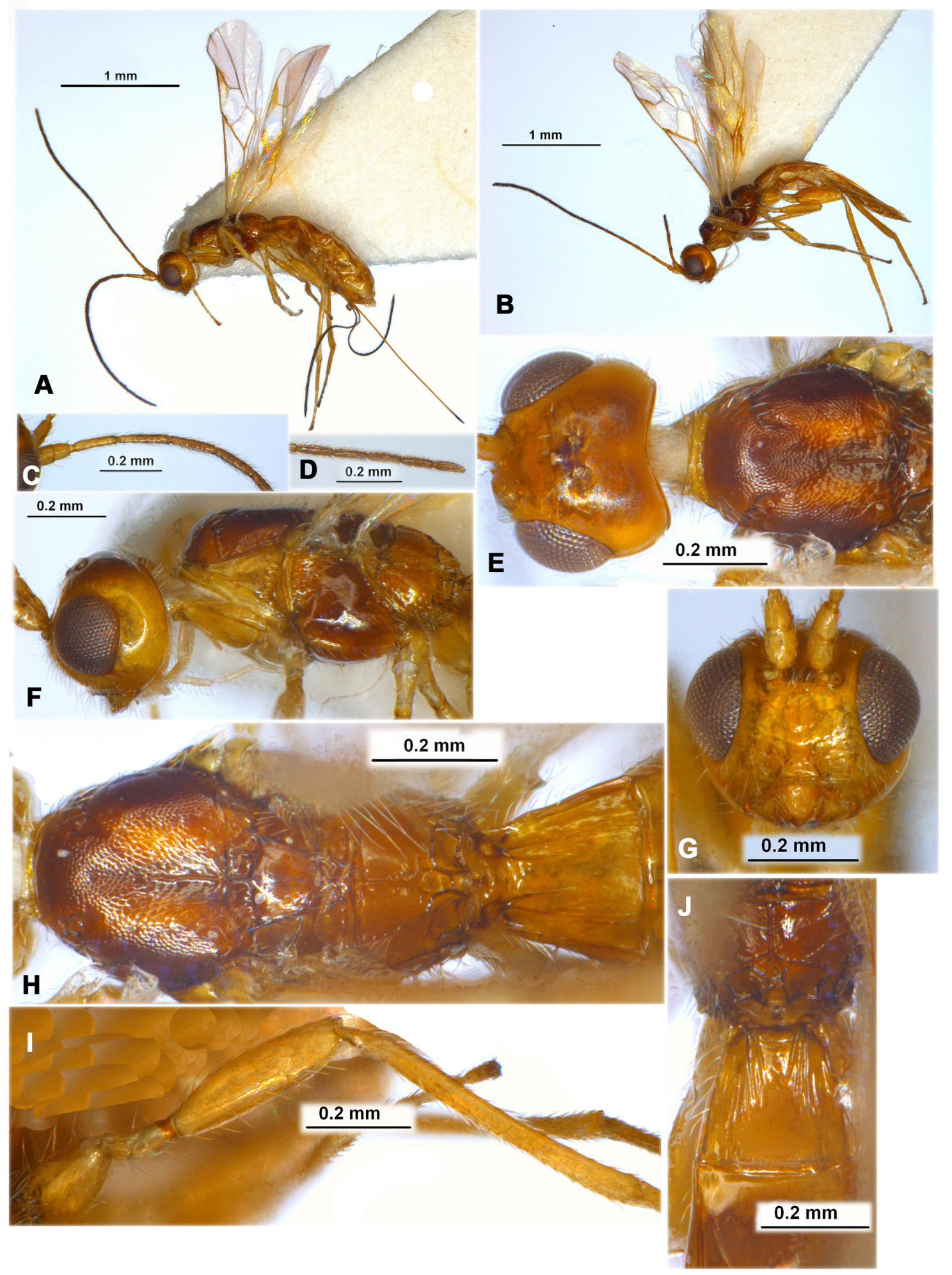

Fig. 63. Aivalykus microaciculatus Ranjith \& Belokobylskij, 2020, paratypes. A, C-J. $q$ (RMNH). B. § (RMNH). A-B. Habitus, lateral view. C. Basal segments of antenna. D. Antennal segments of antenna. E. Head and mesoscutum, dorsal view. F. Head and mesosoma, lateral view. G. Head, front view. H. Mesosoma and first metasomal tergite, dorsal view. . I. Hind leg. J. Propodeum and first metasomal tergite, dorsal view. 
Doryctinae fauna of the Arabian fauna consists of elements from three zoogeographic regions converging on the peninsula, viz., the Afrotropical, Oriental and Palaearctic regions, combined with a large number of Arabian endemisms.

The doryctine genera with species probably adapted to desert and semi-desert habitats are Hecabalodes, with four out of the five species within the genus recorded, Mimodoryctes, with two known species, and Leluthia, with two new species. Furthermore, the large doryctine genus Spathius has five recorded species in the studied area, despite the predominantly dry territories of the peninsula. This result is
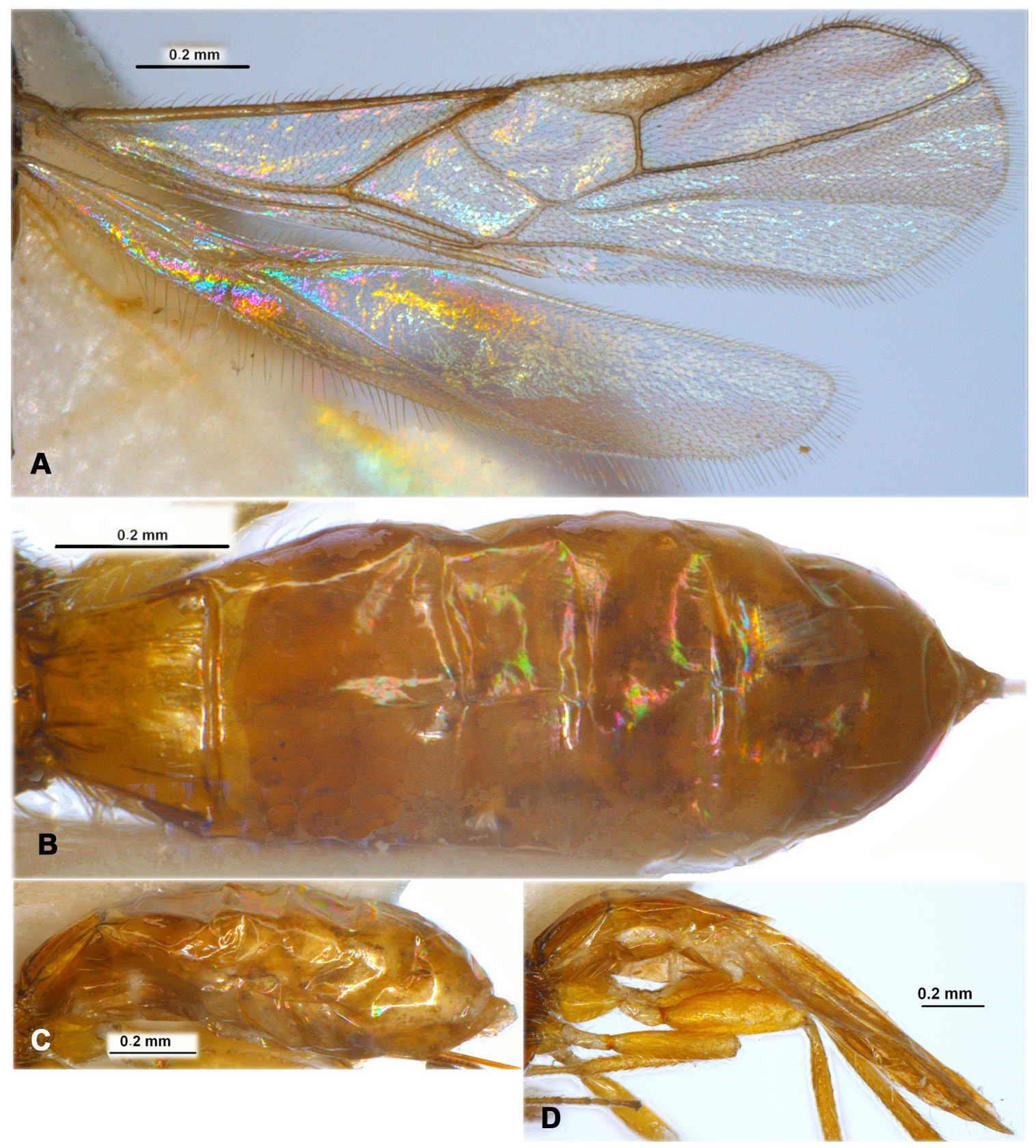

Fig. 64. Aivalykus microaciculatus Ranjith \& Belokobylskij, 2020, paratypes. A-C. $q$ (RMNH). D. § (RMNH). A. Wings. B. Metasoma, dorsal view. C-D. Metasoma, lateral view. 
remarkable because this genus is considerably speciose in the wet tropical forests of the Oriental and Afrotropical regions.

Most of the species of Rhaconotus reported for the studied regions are covered by coarse reticulaterugulose sculpture, viz. Rh. arabicus, Rh. brevicellularis Belokobylskij sp. nov., Rh. magniareolus Belokobylskij sp. nov. and Rh. microexcavatus Belokobylskij sp. nov. Interestingly, Rhaconotus brevicellularis has a clearly shortened radial (marginal) cell of the fore wing, which is distinctive for xerophyte inhabitants. A similar venation is also recorded for two other xerothermic species, Rh. acmaeoderellae and Rh. kerzhneri Belokobylskij, 1985, both described and recorded in the dry territories of Central and South-Western Asia (Belokobylskij 1990).

The discovery of relatively rare genera such as Parana, Parallorhogas, Platyspathius and Hemidoryctes in the fauna of the Arabian Peninsula is also remarkable. The second new species of the former monotypic genus Parana is the first record of this taxon outside of the Indian subcontinent. The trans-tropical genus Hemidoryctes is monotypic, but the discovery of a new subspecies from the Arabian Peninsula characterized by the postfurcal position of the recurrent $(\mathrm{m}-\mathrm{cu})$ vein in fore wing is remarkable. Of special interest is the discovery of an additional species in the mainly subtropical and tropical genera Platyspathius (with two new Arabian species similar to the Afrotropical P. clymene) and Parallorhogas (with a single species having the vertex sculptured and an Afrotropical distribution).

The intensive collection of parasitoids using the different methods employed by A. van Harten across the United Arab Emirates and Yemen is an excellent example of how to create extensive collections of valuable scientific material from territories of the Earth previously hardly investigated.

\section{Acknowledgements}

We are very thankful to Dr Gavin Broad (London, UK), Dr Francisco Javier Peris-Felipo (Basel, Switzerland) and an unknown reviewer for their useful comments on the first version of this manuscript. This work in part was funded by grants given by the Russian Foundation for Basic Research (Project No. 19-04-00027) and the Russian State Research Project No. AAAA-A19-119020690101-6 to SAB.

\section{References}

Ashmead W.H. 1905. New Hymenoptera from the Philippine Islands. Canadian Entomologist 37 (1): 3-8. https://doi.org/10.4039/Ent373-1

Belokobylskij S.A. 1983. Contribution to the knowledge of the genera Heterospilus Hal. and Dendrosotinus Tel. (Hymenoptera, Braconidae) of the USSR. Trudy Vsesoyuznogo Entomologicheskogo Obshchestva [Proceedings of the All-Union Entomological Society] 65: 168-186. [In Russian.]

Belokobylskij S.A. 1990. A review of braconid wasps of the genus Rhaconotus Ruthe (Hymenoptera, Braconidae) of the Palaearctic Region. Entomologicheskoe Obozrenie 69 (1): 144-163. [In Russian.]

Belokobylskij S.A. 1994. The species of the genus Heterospilus Haliday 1836 (Hymenoptera Braconidae) from Vietnam. Tropical Zoology 7 (1): 11-23. https://doi.org/10.1080/03946975.1994.10539237

Belokobylskij S.A. 2001a. New taxa of the braconid subfamilies Doryctinae and Exothecinae (Hymenoptera, Braconidae) from West Palearctic. Entomologicheskoe Obozrenie 80 (2): 451-471. [Entomological Review 81 (7): 749-766.]

Belokobylskij S.A. 2001b. New species of the genera Rhaconotus Ruthe, Ipodoryctes Granger and Arhaconotus Blkb. from the Oriental region (Hymenoptera: Braconidae, Doryctinae). Zoosystematica Rossica 10 (1): 101-162. 
Belokobylskij S.A. 2015. Review of species of the Old World genus Doryctophasmus Enderlein, 1912 (Hymenoptera: Braconidae: Doryctinae). Zootaxa 3985 (4): 541-564.

https://doi.org/10.11646/zootaxa.3985.4.4

Belokobylskij S.A. 2019. Some taxonomical corrections and new faunistic records of the species from the family Braconidae (Hymenoptera) in the fauna of Russia. Proceedings of the Russian Entomological Society 90: 33-53. https://doi.org/10.47640/1605-7678_2019_90_33

Belokobylskij S.A. 2020a. Two new species of the genus Neoheterospilus Belokobylskij, 2006 (Hymenoptera: Braconidae: Doryctinae) from Yemen. Zootaxa 4853 (4): 591-600.

https://doi.org/10.11646/zootaxa.4853.4.8

Belokobylskij S.A. 2020b. Twonew species of the genus Leluthia (Hymenoptera: Braconidae: Doryctinae) from Yemen. Zoosystematica Rossica 29 (2): 284-295. https://doi.org/10.31610/zsr/2020.29.2.284

Belokobylskij S.A. \& Chen X. 2004. The species of the genus Rhaconotus Ruthe, 1854 (Hymenoptera: Braconidae: Doryctinae) from China with a key to species. Annales Zoologici 54 (2): 319-359.

Belokobylskij S.A. \& Maetô K. 2009. Doryctinae (Hymenoptera, Braconidae) of Japan. Fauna mundi 1. Warszawska Drukarnia Naukowa, Warszawa.

Belokobylskij S.A. \& Tobias V.I. 1986. Fam. Doryctinae. In: Medvedev G.S. (ed.) Opredelitel' Nasekomych Evrospeyskoy Chasti SSSR, Pereponchatokrylye [Key to Insects of the USSR European Part. Hymenoptera] 3 (4): 21-72.

Belokobylskij S.A. \& Zaldívar-Riverón A. 2021. Reclassification of the doryctine tribe Rhaconotini (Hymenoptera, Braconidae). European Journal of Taxonomy 741: 1-168.

https://doi.org/10.5852/ejt.2021.741.1289

Belokobylskij S.A., Samartsev K.G. \& Il'inskaya A.S. (eds). 2019. Annotated Catalogue of the Hymenoptera of Russia. Volume II. Apocrita: Parasitica. Proceedings of the Zoological Institute Russian Academy of Sciences Supplement 8. Zoological Institute RAS, St Petersburg.

https://doi.org/10.31610/trudyzin/2019.supl.8.5

Edmardash Y.A., Abu El-Ghiet U.M., Soliman A.M., Al-Fifi Z.I.A. \& Gadallah N.S. 2020. First contribution to the doryctine fauna (Hymenoptera, Braconidae, Doryctinae) of Farasan Archipelago, Saudi Arabia, with new records and the description of a new species. ZooKeys 977: 41-74. https://doi.org/10.3897/zookeys.977.56314

Erichson W.F. 1837. Bericht über die wissenschaftlichen Leistungen in der Entomologie während des Jahres 1836. Archiv für Naturgeschichte 2: 281-339.

Fernández-Triana J. \& van Achterberg C. 2017. Order Hymenoptera, family Braconidae Subfamily Microgastrinae from the Arabian Peninsula. In: van Harten A. (ed.) Arthropod Fauna of the United Arab Emirates 6: 275-321. Department of the President's Affairs, Abu Dhabi. Available from https://repository.naturalis.nl/pub/643511 [accessed 11 Jun. 2021].

Fischer M. 1962. Neue Braconiden-Parasiten von schädlichen Insekten (Hymenoptera). Zeitschrift für Angewandte Entomologie 49 (3): 297-312. https://doi.org/10.1111/j.1439-0418.1961.tb02868.x

Fischer M. 1980 Taxonomische Untersuchungen über Doryctinae aus der Odontobracon Verwandtschaft (Hymenoptera, Braconidae). Annalen des Naturhistorischen Museums in Wien 83: 547-572. Available from https://www.jstor.org/stable/41768825 [accessed 11 Jun. 2021].

Granger C. 1949. Braconides de Madagascar. Mémoires de l'Institut scientifique de Madagascar (A) 2: $1-428$. 
Jasso-Martínez J.M., Belokobylskij S.A. \& Zaldívar-Riverón A. 2019. Molecular phylogenetics and evolution of generic diagnostic morphological features in the doryctine wasp tribe Rhaconotini (Hymenoptera: Braconidae). Zoologischer Anzeiger 279: 164-171.

https://doi.org/10.1016/j.jcz.2019.02.002

Lelej A.S. \& van Harten A. 2006. A review of the Mutillidae (Hymenoptera) of Yemen. Zootaxa 1226: 1-50. https://doi.org/10.11646/zootaxa.1226.1.1

Marsh P.M., Wild A.L. \& Whitfield J.B. 2013. The Doryctinae (Braconidae) of Costa Rica: genera and species of the tribe Heterospilini. ZooKeys 347: 1-474. https://doi.org/10.3897/zookeys.347.6002

Marshall T.A. (1888)1890. Les Braconides. In: André E. (ed.) Species des Hyménoptères d'Europe et d'Algérie 4: 1-609. Chez l'auteur, Beaune. https://doi.org/10.5962/bhl.title.10281

Nixon G.E.J. 1939. New species of Braconidae (Hymenoptera). Bulletin of Entomological Research 30: 119-128. https://doi.org/10.1017/S0007485300004430

Nixon G.E.J. 1941. The Indian and African species of Rhaconotus Ruthe (Hym., Braconidae). Annals and Magazine of Natural History (11) 7: 473-503. https://doi.org/10.1080/03745481.1941.9723712

Nixon G.E.J. 1943. A revision of the Spathiinae of the old world (Hymenoptera, Braconidae). Transactions of the Royal Entomological Society of London 93: 173-456.

https://doi.org/10.1111/j.1365-2311.1943.tb00434.x

Polaszek A., Fitton M.G., Bianchi G. \& Huddleston T. 1994. The parasitoids of the African white rice borer, Maliarpha separatella Ragonot (Lepidoptera: Pyralidae). Bulletin of Entomological Research 84: 65-89. https://doi.org/10.1017/S0007485300032247

Ranjith A.P., Belokobylskij S.A., Sureshan P.M. \& Nasser M. 2020. The genus Aivalykus Nixon, 1938 (Hymenoptera: Braconidae: Doryctinae) with description of a new species from India and Arabian Peninsula. Zootaxa 4822 (2): 269-276. https://doi.org/10.11646/zootaxa.4822.2.8

Shenefelt R.D. 1975. Braconidae 8. Exothecinae, Rogadinae. In: Ferriere Ch. \& van der Vecht J. (eds) Hymenopterorum Catalogus (nova editio) 12: 1115-1262.

Shenefelt R.D. \& Marsh P.M. 1976. Braconidae 9. Doryctinae. In: Ferriere Ch. \& van der Vecht J. (eds) Hymenopterorum Catalogus (nova editio) 13: 1263-1424.

Szépligeti G. 1914. Afrikanische Braconiden des Königl. Zoologischen Museums in Berlin. Mitteilungen aus dem Zoologischen Museum in Berlin 7: 153-230.

Tang P., Belokobylskij S.A., He J.-H. \& Chen X.-X. 2013. Heterospilus Haliday, 1836 (Hymenoptera: Braconidae, Doryctinae) from China with a key to species. Zootaxa 3683 (3): 201-246.

https://doi.org/10.11646/zootaxa.3683.3.1

Tobias V.I. 1962. Two new species of braconids (Hymenoptera) from Central Asia and their relationship with the African fauna. Proceedings of Zoological Institute of the USSR Academy of Sciences 30: 271277. [In Russian.]

van Achterberg C. 1993. Illustrated key to the subfamilies of the Braconidae (Hymenoptera: Ichneumonoidea). Zoologische Verhandelingen 283: 3-189.

van Achterberg C. 2003. The West Palaearctic species of the genera Gildoria Hedqvist and Platyspathius Viereck, with keys to the species (Hymenoptera: Braconidae: Doryctinae). Zoologische Mededelingen 77 (15): 267-290.Available from https://repository.naturalis.nl/pub/216152 [accessed 8 Aug. 2021].

van Achterberg C. 2010. Order Hymenoptera, family Braconidae. Genus Microtypus Ratzeburg (Hymenoptera: Braconidae: Microtypinae). In: van Harten A. (ed.) Arthropod Fauna of the United Arab Emirates 3: 381-387. Department of the President's Affairs, Abu Dhabi. 
van Achterberg C. 2011. Order Hymenoptera, family Braconidae. The subfamily Agathidinae from the United Arab Emirates, with a review of the fauna of the Arabian Peninsula. In: van Harten A. (ed.) Arthropod Fauna of the United Arab Emirates 4: 286-352. Department of the President's Affairs, Abu Dhabi.

van Achterberg C. 2014. Order Hymenoptera, family Braconidae. The subfamily Homolobinae from the United Arab Emirates, with a review of the fauna of the Arabian Peninsula. In: van Harten A. (ed.) Arthropod Fauna of the United Arab Emirates 5: 426-433. Department of the President's Affairs, Abu Dhabi.

van Achterberg C. \& Polaszek A. 1996. The parasites of cereal stem borers (Lepidoptera: Cossidae, Crambidae, Noctuidae, Pyralidae) in Africa, belonging to the family Braconidae (Hymenoptera: Ichneumonoidea). Zoologische Verhandelingen 304: 1-123.

van Harten A. 2009. Introduction. In: van Harten A. (ed.) Arthropod Fauna of the United Arab Emirates 2: 11-16. Department of the President's Affairs, Abu Dhabi.

Viereck H.L. 1914. Type species of the genera of Ichneumon flies. Bulletin of the United States National Museum 83: 1-186. https://doi.org/10.5479/si.03629236.83.1

Wilkinson D.S. 1927. Eight new species of Braconidae. Bulletin of Entomological Research 18: 33-46. https://doi.org/10.1017/S0007485300019659

Wilkinson D.S. 1929. New parasitic Hymenoptera and notes on other species. Bulletin of Entomological Research 20 (1): 103-114. https://doi.org/10.1017/S000748530002099X

Yu D.S.K., van Achterberg C. \& Horstmann K. 2016. Taxapad 2016, Ichneumonoidea 2015. Database on flash-drive. Nepean, Ontario.

Zaldívar-Riverón A., Belokobylskij S.A., León-Regagnon V., Briceño R. \& Quicke D.L.J. 2008. Molecular phylogeny and historical biogeography of the cosmopolitan parasitic wasp subfamily Doryctinae (Hymenoptera: Braconidae). Invertebrate Systematics 22: 345-363.

https://doi.org/10.1071/IS07028

Zaldívar-Riverón A., Belokobylskij S.A., Meza-Lázaro R., Pedraza-Lara C., García-París M. \& Meseguer A.S. 2018. Species delimitation, global phylogeny and historical biogeography of the parasitoid wasp genus Spathius (Braconidae: Doryctinae) reveal multiple Oligocene-Miocene intercontinental dispersal events. Zoological Journal of the Linnean Society 182 (4): 723-734.

https://doi.org/10.1093/zoolinnean/zlx050

Manuscript received: 18 January 2021

Manuscript accepted: 13 April 2021

Published on: 30 August 2021

Topic editor: Nesrine Akkari

Topic editor: Gavin Broad

Desk editor: Pepe Fernández

Printed versions of all papers are also deposited in the libraries of the institutes that are members of the EJT consortium: Muséum national d'histoire naturelle, Paris, France; Meise Botanic Garden, Belgium; Royal Museum for Central Africa, Tervuren, Belgium; Royal Belgian Institute of Natural Sciences, Brussels, Belgium; Natural History Museum of Denmark, Copenhagen, Denmark; Naturalis Biodiversity Center, Leiden, the Netherlands; Museo Nacional de Ciencias Naturales-CSIC, Madrid, Spain; Real Jardín Botánico de Madrid CSIC, Spain; Zoological Research Museum Alexander Koenig, Bonn, Germany; National Museum, Prague, Czech Republic. 